\title{
Microfluidic Bubble Logic
}

\author{
by
}

\section{Manu Prakash}

B.Tech. Computer Science and Engineering, Indian Institute of Technology, Kanpur, 2002

S.M. Media Arts and Sciences, Massachusetts Institute of Technology, 2004

Submitted to the Program in Media Arts and Sciences,

School of Architecture and Planning, in partial fulfillment of the requirements for the degree of Doctor of Philosophy in Media Arts and Sciences at the Massachusetts Institute of Technology

\section{September 2008}

MASSACHUSETS INSTHTTE
OF TECHNOLOGY
JAN 062009
LIBRARIES

(C) Massachusetts Institute of Technology. All rights reserved.

$$
\cap \Lambda \sim
$$

Signature of Author

Program in Media Arts and Sciences

July 2008

Certified by

Director, Center for Bits and Atoms Professor, Program in Media Arts and Sciences

Accepted by

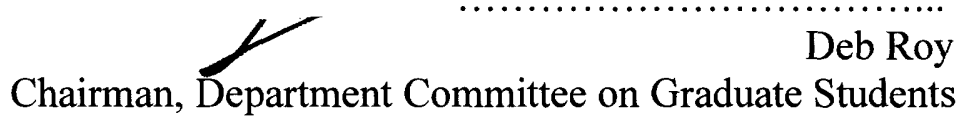




\title{
Microfluidic Bubble Logic
}

\author{
by \\ Manu Prakash
Submitted to the Program in Media Arts and Sciences, School of Architecture and Planning, on September, 2008, in partial fulfillment of the requirements for the degree of Doctor of Philosophy in Media Arts and Sciences

\begin{abstract}
In this thesis, I propose a new paradigm in computing where bits can simultaneously transport and manipulate materials and information. Information representation is invariably physical. Though this insight is fundamental to understanding the physical limits of computation, it has never been exploited as a scheme for material manipulation. Bringing together notions from computer science and fluid dynamics, I present a new logic family "Bubble Logic" capable of both universal computation and programmable material manipulation in an all-fluidic two-phase system. This removes the distinction between materials and mechanisms to control them, bringing the programmability of the digital world into the physical world - with a wide range of promising applications in biotechnology, highthroughput screening, genomics and fluidic control systems for soft robotics, printing and digital fabrication.
\end{abstract}

Microfluidics, the art of handling nano-to pico-liter volume fluids, is leading to a revolution in large-scale automation of biology and analytical chemistry. However, current lab-on-chip technologies are dependent on external macro-scale control elements, thus requiring a lab to run the chip. Bubble logic provides a droplet-level, internal, inherently digital flow control mechanism at $\mathrm{kHz}$ frequencies with no moving parts or off-chip components. Nonlinearity is introduced in an otherwise linear, reversible, low Reynolds number flow via bubble-tobubble hydrodynamic interactions. I demonstrate bubble logic AND/OR/NOT gates, a toggle flip-flop, a ripple counter, a timing restoration device, a ring oscillator, a bistable valve and an on-demand bubble generator. These show the nonlinearity, gain, bistability, synchronization, cascadability, feedback and programmability required for scalable universal computation and control. The representation used in this thesis makes possible encapsulation and manipulation of a large variety of micro-to nano-scale materials including single molecules like DNA or proteins, live cells, liquid crystals, nano-particles and other biological and chemical reagents. Bubble logic provides a scheme to transport, store and operate on this new class of "digital materials" in an integrated, high-throughput fashion.

Furthermore, microfluidics has also been extensively employed in biological systems. This thesis describes the discovery of two new physical fluid dynamic mechanisms motivated by a 
common theme of microfluidics in biology. Firstly, I describe a new superhydrophobic waterrepelling surface that has a characteristic of directional anisotropy to fluid resistance. The discovery, made while studying the integument of water-walking insects, helps rationalize the origin of thrust and hence propulsion of water-walking insects on a fluid interface. Secondly, this thesis uncovers a new physical mechanism for directed droplet transport, which I term "Capillary ratchet". Discovered in a class of surface feeding shorebirds, it is the only physical mechanism that is known to exploit contact angle hysteresis for fluid transport. Capillary ratchet is a promising candidate for implementing global clocking for integrated microfluidic devices.

Thesis Supervisor: Neil Gershenfeld

Title: Director, Center for Bits and Atoms, Professor of Media Arts and Sciences, Program in Media Arts and Sciences 


\section{Microfluidic Bubble Logic}

\section{Manu Prakash}

Submitted to the program in Media Arts and Sciences, School of Architecture and Planning in partial fulfillment of the requirements of the Degree of Doctor of Philosophy.

Massachusetts Institute of Technology

September 2008

Neil Gershenfeld

Professor of Media Arts and Sciences

Thesis Advisor

Massachusetts Institute of Technology

Alex Slocum

Thesis Reader

Pappalardo Professor of Mechanical Engineering

Department of Mechanical Engineering

Massachusetts Institute of Technology

\section{Johh W. M. Bush}

Associate Professor of Applied Mathematics

Thesis Reader

Department of Mathematics

Massachusetts Institute of Technology

Edward S. Byoden

Thesis Reader

Assistant Professor of Media Arts and Sciences and

Department of Biological Engineering

Massachusetts Institute of Technology 


\section{Acknowledgements}

During my stay at MIT, I have been inspired, excited, shocked and mentored by many people - more than will fit a page. I am forever thankful to all of them for sharing the spirit of MIT with me.

Creativity begins with freedom to explore with no bounds. In my academic life, one person who has shown me this path is my advisor - Neil Gershenfeld. His passionate pursuit for bringing down the boundaries between computer science and physical science - taught me how to make computers disappear into the physical world. His vision and tireless implementation of "FabLabs" will forever inspire me to bridge the "fabrication divide" in developing world. I am forever indebt to him for his tremendous support and mentorship.

I want to also thank -

John Bush - for showing me how research can be both enjoyable and stimulating; with the perfect mix of work and play - teaching me the importance of good writing and making me a part of the "fluids community" at MIT.

David Quere - for showing me the power of scaling laws and a wonderful collaboration; and an unforgettable visit to Paris.

Alex Slocum - for showing me the true MIT spirit: forever full of energy and pursuing inventive (crazy) ideas and being a great mentor.

Stephen Quake - for his vision and mentorship and showing me how technological advances can truly impact a wider variety of fields.

Shuguang Zhang - for showing me the importance of asking "big questions".

Saul Griffith - for being a great lab-mate, a fantastic friend and expanding my personal definition of both adventure and sanity.

Brian Chow - for being the local chemistry wiz and forever ready for a quick cup of coffee (which often turned into long discussions on philosophy of science).

Ara Kanian, Amy Sun, Sun Xu, Kenneth Cheung, Luis Lafuente, Kailiang Chen, Yael Maguire, Matt Reynolds, Rehmi Post, Ben Retch and Ben Vigoda for making PHM such a fun place to work - and being endless resource of ideas and sanity check.

David Kong, Vikrant Agnihotri, Jaebum Joo, Peter Carr, Thomas Burg and folks from Mo-machines and Nano-media for putting together and sharing all kinds of fabrication resources. 
Jeff Aristoff, Pedro Reis, Sunny, David Hu and friends at the MIT Math Department - for "fluids lunch" talks and welcoming me in that community.

It is difficult to overstate my gratitude for John DiFrancesco, Susan Bottari, Joe Murphy, Kelly Maenpaa, Sherry Lassister and MTL staff for making all possible resources available.

Center for Bits and Atoms - for providing me a tremendous opportunity to be a part of Fablabs all around the world - from above the arctic circle in Norway to South Africa, India and Ghana. The Fab community has forever enriched my view of what appropriate technology can do for developing economies.

Ed Boyden, Scott Manalis, Christophe Clanet, Luca Turin, Andreas Mershin and George Church - for stimulating discussions on the future of technology, role of science in society and everything else under the sun.

I most sincerely acknowledge the Public Service Center at MIT, for giving the driving wheel of social development projects at MIT, in the hands of the students.

My Mom - for teaching me the value of hard work and always pursuing my dreams - it would have never been possible without your never-ending support.

My Dad - for instilling in me the value of conserving resources and the importance of giving back to society.

My Brother - taking the blame of failed experiments and being a great sport.

And finally Akshi - for always being there for me. 


\section{Contents}

$\begin{array}{ll}\text { A.bstract } & 1\end{array}$

1 Introduction 19

1.1 Information is Physical . . . . . . . . . . . . . . . 19

1.2 Manipulating materials at small length scales . . . . . . . . . . . . . 21

1.3 All-fluidic logic and control . . . . . . . . . . . . . . . . . . 23

1.3.1 High Reynolds number . . . . . . . . . . . . . . . . . . 23

1.3.2 Low Reynolds number . . . . . . . . . . . . . . . . . . 25

1.4 Compartmentalization: Droplet based digital materials . . . . . . . . . . . . . 31

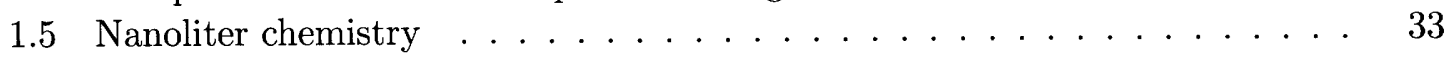

1.6 Bio-inspired Design . . . . . . . . . . . . . . . . . . . . . 35

1.7 Summary of Major Results . . . . . . . . . . . . . . . . . . 36

1.8 Thesis Structure and Overview . . . . . . . . . . . . . 37

2 Microfluidic Droplet Networks $\quad 43$

2.1 Low Reynolds number Stokes flow . . . . . . . . . . . . . . . . . . . . 44

2.2 Resistance of a droplet in a channel . . . . . . . . . . . . . . . . . 46

2.2.1 Complete vs. Partial Wetting . . . . . . . . . . . . . . . 46

2.2.2 Resistance of a clean bubble: Inviscid dispersed phase . . . . . . . . 46

2.2 .3 Effect of viscosity . . . . . . . . . . . . . . . . . . . 48

2.2 .4 Droplets at a $\mathrm{T}$ junction . . . . . . . . . . . . . . 50

2.2 .5 Numerical formulation . . . . . . . . . . . . . . 51

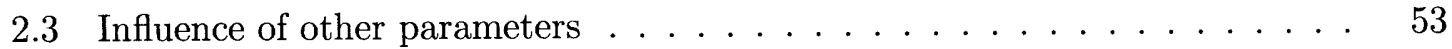

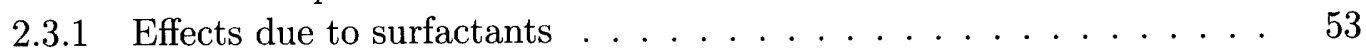

2.3 .2 Instabilities due to low surface tension . . . . . . . . . . . . . 54

2.3 .3 Gravity ............................... 57

3 Microfluidic bubble logic $\quad 61$

3.1 Abstract . . . . . . . . . . . . . . . . . . . 61

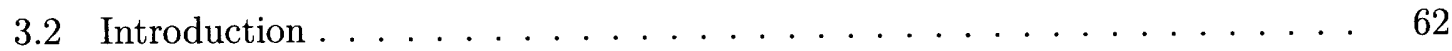

3.3 Microfluidic channel networks . . . . . . . . . . . . . . 63

3.4 Universal Logic Gates . . . . . . . . . . . . . . . . . . . . . . . . 64

3.5 Bistability . . . . . . . . . . . . . . . . . . . 67

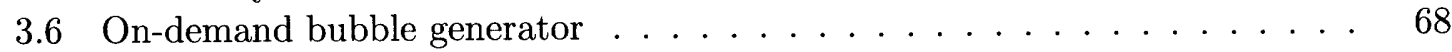

3.7 Cascadability and feedback . . . . . . . . . . . 68 


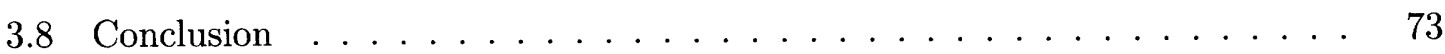

4 Device Design Principles $\quad 79$

4.1 Introduction . . . . . . . . . . . . . . . . . . 79

4.2 Droplets at a junction and bypass channels . . . . . . . . . . . 80

4.3 Microfluidic Logic Gates - AND/NOT gate . . . . . . . . . . . . . . 81

4.4 Bistable one-bit memory . . . . . . . . . . . . . . . . . . . . 84

4.5 Synchronization in microfluidic circuits . . . . . . . . . . . . 86

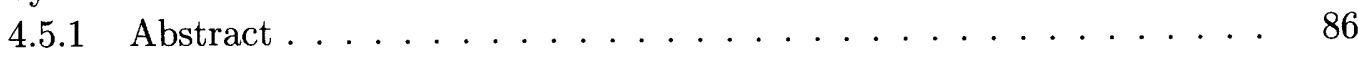

4.5 .2 Introduction . . . . . . . . . . . . . . . . 88

4.5.3 Microfluidic Ladder Network synchronizer (LNS) . . . . . . . . . . . 88

4.5.4 Results and Discussion . . . . . . . . . . . . . . . . . . . . 91

4.6 Design Tools . . . . . . . . . . . . . . . . . . . . 93

4.7 Appendix: Symbolic evaluation of resistance networks . . . . . . . . 98

5 Unidirectional superhydrophobic surfaces 103

5.1 Abstract . . . . . . . . . . . . . . . . . . 103

5.2 Introduction . . . . . . . . . . . . . . . . . . . . 104

5.3 Contact line adhesion . . . . . . . . . . . . . . . . . 104

5.4 Experiments . . . . . . . . . . . . . . . . . . 107

5.4 .1 Force measurement . . . . . . . . . . . . . . . . . . 109

5.5 Methods . . . . . . . . . . . . . . . . . . . 111

5.5 .1 SEM imaging . . . . . . . . . . . . . . . . . . . 113

5.5 .2 Inverted microscopy . . . . . . . . . . . . . . . 113

5.5 .3 Preparation of tarsal leg samples . . . . . . . . . . . . . . . . 113

5.5 .4 Contact force measurements . . . . . . . . . . . . . . . . . 114

5.5 .5 Droplet impact experiments . . . . . . . . . . . . . . . . 115

5.5.6 High speed imaging of the rowing stroke . . . . . . . . . . . 115

5.6 Conclusion . . . . . . . . . . . . . . . . 115

6 Capillary ratchets $\quad 123$

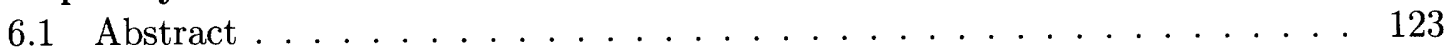

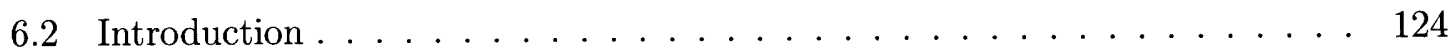

6.3 Experiments . . . . . . . . . . . . . . . . . . . . 125

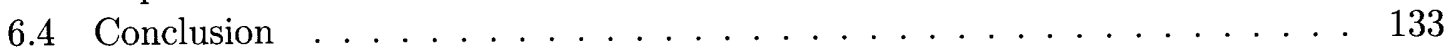

6.5 Appendix: Surface Evolver Source Code . . . . . . . . . . . . . 133

$7 \quad$ Future work and conclusion $\quad 145$

7.1 Advantages . . . . . . . . . . . . . . . . . . . 145

7.2 Combinatorial chemistry . . . . . . . . . . . . . . . . 146

7.3 Dynamic Micro-arrays . . . . . . . . . . . . . . . . . . . . 149

7.4 Surface bound detection and analysis systems . . . . . . . . . . . 152

7.4.1 Microfluidic Olfactory receptor based odorant detection . . . . . . . 153

7.4.2 Odorant control for patterned stimulation of olfactory receptors . . . 155

7.4 .3 Artificial Sniffing . . . . . . . . . . . . . . . . . . 156

7.5 Microfluidic Lock-in amplifier . . . . . . . . . . . . . . . 157 
7.6 Micro-droplet processors . . . . . . . . . . . . . . . . 160

7.7 Fluidic control . . . . . . . . . . . . . . . . . . . . . 162

7.7 .1 Soft robotics . . . . . . . . . . . . . . . 162

7.7 .2 Multi-material Print heads . . . . . . . . . . . . . . 164

7.7 .3 Fluidic displays . . . . . . . . . . . . . . . . . . . . 164

7.7.4 Programmable nano-liter drug delivery . . . . . . . . . . . . . 165

7.8 Digital Fabrication . . . . . . . . . . . . . . . . . 167

7.9 Future directions $\ldots \ldots \ldots \ldots \ldots \ldots \ldots$

7.10 Conclusion . . . . . . . . . . . . . . . 171 


\section{List of Figures}

1-1 Reversible logic scheme implemented as collision of hard balls, where presence or absence of a ball represents 0 or 1 . Reflecting mirrors and geometrical arrangements govern the interaction patterns for the balls. . . . . . . .

1-2 Conventional liquid handling robots are large, bulky, slow, costly to operate and require micro-liters of expensive reagents for individual screening condition; thus making it financially non-viable for screening millions of conditions or performing complex assays in a few seconds. Inset depicts a micro-titer plate for chemical storage. . . . . . . . . . . . . . . .

1-3 Integrated fluidic circuits (IFC) employing "Nano-flex" elastomeric valves. (a) Photograph of a $48 \times 48$ dynamic array micro-chip using elastomeric valves. (b)Photograph of a protein crystallization chip (TOPAZ, fluidigm) with clear elastomeric IFC integrated in a carrier for liquid delivery. (c,d) Microchip with reaction loop, integrated valves and pumps for on-chip synthesis of radiolabeled fluorine as cancer probe. . . . . . . . . . . . . .

1-4 (a) High Reynolds number fluidic logic gates from the 06's with varying size from meter scales vortex valves to milli-metric scale proportional amplifier. (b) Schematic description of fluidic logic gate with equivalent electrical analogs depicted. (c) Vortex ring based jet-amplifier with output jet stream controlled by inlet control lines. (d,e) Integrated fluidic logic gates with a schematic integrated control circuit and an all-fluidic display from UNIVAC.

1-5 Groisman et al. have shown memory and control elements using non-newtonian fluids. Extension and relaxation of polymer molecules results in asymmetric flow behavior in an expansion and contraction geometry, also depicted by pressure vs. flow-rate characteristic curve for a given channel geometry. . .

1-6 Fluidic valves based on flexible membranes sandwiched between glass wafers. The valve can be turned on and off via negative pressure and can be latched in either on and off position. A simple XOR gate formed using six pneumatic valves. . . . . . . . . . . . . . . . . . .

1-7 Comparison of a chip-scale detection technologies with convention liquid handling equipment required for automated operation of such a device. Usually, large liquid handling and control equipment is required to handle samples on micro-chips, leading to the notion of a "lab around a chip". . . . . . . . . 
1-8 Compartmentalization in natural and artificial systems. (a) Giacomo Ciamician (1857-1922), father of green chemistry, on the roof of the Chemical Institute in Bologna among the flasks he is exposing to solar light running experiments in combinatorial chemistry. (b) SEM images of E-coli, roughly a $1 \mu \mathrm{m}$ in length serve as individual biological compartments. (c) Monodisperse fluid droplets, $40 \mu \mathrm{m}$ in size generated using microfluidic emulsion techniques, can act as artificial cells capable of performing chemical reactions in confined volumes. . . . . . . . . . . . . . . . . . 32

1-9 Encapsulation of natural and synthetic materials in compartmentalized droplets. A large array of nano-bio materials can be encapsulated using the prescribed scheme including bio-molecules, single organelles, single cells, whole organisms, protein crystals, liquid crystals and so on. . . . . . . . . .

2-1 Channel network with complex topology and connectivity found in animate and in-animate natural systems.

2-2 Fluid filled in a tube displaced by another non-wetting fluid. Three regions depict various dominant forces governing the interface shape for the dispersed

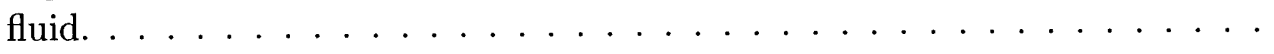

2-3 Pressure drop due to a train of droplets in a channel. . . . . . . . . .

2-4 Interface shape due to an inviscid bubble traveling in a channel, with six distinct regions marked by the trailing and the leading edge of the bubble.

2-5 Surfactant distribution on the interface of a inviscid bubble in a wetting fluid. 55

3-1 Universal microfluidic bubble logic. A bubble in a channel represents a bit. $\mathrm{A}$ and $\mathrm{B}$ mark the $\mathrm{T}$ junction bubble input ports to the devices (planar 2D geometry, channel height $70 \mu \mathrm{m}$ ) which are driven by a constant water input flow (with $2 \% \mathrm{w} / \mathrm{w}$ Tween 20 surfactant) via syringe pumps (Kent Scientific) and constant pressure driven nitrogen $\left(P_{0}=0.5 \mathrm{psi}\right) . \ldots \ldots \ldots$

3-2 Bistability: Toggle flip-flop with one bit memory. The device stores a bubble (A) indefinitely against a constant flow $(Q=0.25 \mu \mathrm{l} / \mathrm{sec}$ water with $2 \% \mathrm{w} / \mathrm{w}$ Tween 20 , driven by a syringe pump, Kent Scientific) in an energy well until a toggle signal arrives. . . . . . . . . . . . . . .

3-3 Programmable on-demand electro-thermal bubble generator. The device consists of a $50 \mu \mathrm{m}$ wide platinum micro-heater $(R=95 \Omega, 200 \mathrm{~nm}$ thick platinum) followed by a $2 \mu \mathrm{m}$ silicon dioxide dielectric barrier. The heater is embedded below a modified, planar flow focusing geometry . . . . . . .

3-4 Feedback, cascadability and synchronization. Three AND gates are connected in a ring configuration via three delay lines to form a ring oscillator

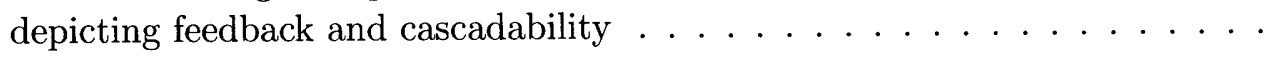

4-1 Schematic and reduced model representation of a spit channel junction and split channel junction with a bypass channel. The effective resistance is depicted to show the variation and non-linear behavior of the channel network as droplets pass through the same. 
4-2 Schematic representation of AND/NOT gate geometry. Reduced order model of the logic gate is developed using non-linear resistor network represented as $R_{i}$ for hydraulic resistance of a channel, $Q_{i}$ for flow rate in a channel and $P_{0}$ for outlet pressure. Schematic representation of the truth table for $\operatorname{NOT}(A) . B$ and $A . B$ is shown below. . . . . . . . . . .

4-3 (A) Schematic of a bistable one-bit memory unit implemented in microfluidic bubble logic. The device is designed to store an arriving droplet until a toggle signal is applied to the inlet line. The maximum flow rate that can be applied to the device is given by $Q_{m} a x$. (B) A reduced order model for the device with a resistive network model is shown in schematic. . . . . . . . . .

4-4 (A) Surface energy plot for a single bubble traversing the toggle flip-flop geometry vs. time. The large minima in the middle refers to the minimum energy well formed at the two lobes. (B) Bifurcation diagram for the toggle flip-flop geometry depicting a sudden transition from bubble breakup to the onset of bistablity. Plot of difference in bubble volume in the two lobes (volume partition of the bubble) is plotted against non-dimensional size of input bubble. . . . . . . . . . . . . . . . . .

4-5 (A) Schematic of synchronization geometry. Bubbles are introduced into the channels at a constant frequency from left to right using a flow-focusing bubble generator operating at a constant flow-rate. (B) Passive synchronization of bubbles in top and bottom channels (width $=100 \mu \mathrm{m}$ ) with interconnected channels $(\mathrm{K}=10$, width $=50 \mu \mathrm{m})$ operating at a constant flow rate of $\mathrm{Qw}=0.5$ $\mu \mathrm{l} / \mathrm{sec}$. Timing restoration of $10 \mathrm{~ms}$ is achieved in $40 \mathrm{~ms}$. Scale bar $100 \mu \mathrm{m}$.

4-6 (A) Time series of a single bubble passing through a ladder network. The bubble slows down as it enters the inter-connected segment. Oscillations in the bubble velocity can be seen whenever the bubble crosses the interconnected vertical channel. (B) Dynamics of continuous synchronization at $5 \mathrm{~Hz}$. (C) Plot of experimental data of distance between the two bubbles vs time with an exponential fit. Inset depicts a log linear plot of decreasing distance with time. . . . . . . . . . . . . . . . . .

4-7 Schematic of a 4-bar ladder network for bubble synchronization, driven by constant flow $Q$ at the inlet and constant pressure $P_{o}$ at the outlet. The plot shows a time vs. distance plot for the 4-bar ladder network with $\bar{r}=8, \bar{R}_{b}=$ $4, L_{2}=1 / 4 L_{1} \ldots \ldots \ldots \ldots \ldots \ldots \ldots \ldots \ldots \ldots \ldots \ldots \ldots \ldots \ldots$

4-8 Variation of traversal velocity of a single bubbles in a ladder network characterized based on the dimensionless resistance of the bypass channels $\bar{r}=r / R$ where $R$ is the resistance of the horizontal channels, $L$ is the length of the inlet and outlet channels and $x$ is the distance travelled by the droplet from the start of the network. As can be observed, for large values of $\bar{r}>20$, the effect of the ladder network to the bubble velocity reduces drastically. For $\bar{r} \rightarrow \infty$, the bypass connections can be assumed to be non-existent. . . . . .

4-9 Effect of $R_{b}$ and $r / R$ on a single bubble traveling through a ladder network. As can be seen, high values of $R_{b} / R$ slows down the bubble significantly. For large values of $r / R$, the ladder network is ineffective in slowing down a bubble. 95 
4-10 (A) Resistor network model of a synchronizer implemented with time varying non-linear resistors. The state of the system can be completely defined by three independent parameters; $\mathrm{Q}$ (inlet flow-rate), $\mathrm{N}$ and $\mathrm{M}$ (location of bubble in upper and lower channel). (B) Spice simulation of the resistor network model for all possible states of the LNS $\left(R_{b}=50 R\right)$. As is evident; relative velocity of bubble in upper channel (at $M$ ) with respect to velocity of bubble in lower channel (at $\mathrm{N}$ ) is positive for $\mathrm{M}>\mathrm{N}$ negative for $\mathrm{M}<\mathrm{N}$; resulting in synchronization of the bubbles. For values $M=N$, the relative velocity (speed of separation, $\Delta u=0) \ldots \ldots \ldots$

4-11 Schematic description of design and simulation tools for microfluidics similar to SPICE. A given set of fluidic channel geometries re converted to non-linear resistor network models that in turn are modeled using reduced order models for constitutive parts. A library of parts is maintained that can be called in the design tool for modular design. . . . . . . . . . . . .

5-1 Scanning Electron Microscope (SEM) images of the rowing leg of the water strider (Gerris remigis). (a) The strider's legs are covered by an array of hairs tilted towards the leg tip. (b) The hairs have characteristic length 20 $\mu \mathrm{m}$, radius $1 \mu \mathrm{m}$ and are marked by longitudinal nanogrooves $(\mathbf{c}, \mathbf{d})$ roughly

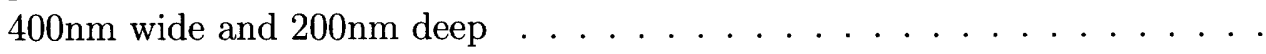

5-2 Brightfield microscope images of water-walking arthropods in a non-wetting Cassie state, captured with an inverted microscope (Zeiss LSM Pascal). a) A live Microvelia standing on the water surface grooming its water-repellent legs. b) A closer look at one of the supporting feet shows that contact between the cuticle and water surface arises exclusively through pin-holes (dark spots) associated with individual hairs. c) Contact line of the leg of a live water strider (Gerris remigis) standing on the water surface. d) Magnification of the marked region reveals that contact between insect and fluid arises exclusively through a single row of hairs. Scale bars: a) $1 \mathrm{~mm}$; b) $100 \mu \mathrm{m}$; c) $250 \mu \mathrm{m}$; d) $100 \mu \mathrm{m}$.

5-3 The interaction of insect cuticle with a translating water droplet. a) For drop motion perpendicular to the leg, severe drop deformations indicate large adhesive forces. b) The drop moves with relative ease towards the leg tip, with little distortion of either hair or interface. c) For motion against the grain of the tilted hairs, the interface is snagged on the elastic hairs and drawn out into a thin sheet evident as a white triangle. d) Individual hairs are deflected by capillary forces as the drop advances against the grain. Scale bars a) $50 \mu \mathrm{m}$; b) $100 \mu \mathrm{m}$; c) $100 \mu \mathrm{m}$; d) $10 \mu \mathrm{m} . \ldots \ldots \ldots$. . . . . . . . . 
5-4 Direct measurements of the adhesive forces generated by a water droplet interacting with the driving leg of a water strider. a) The experimental apparatus. b) The evolution of the force per unit length on the leg as the water droplet is translated past it in directions perpendicular to the leg (green), and parallel to the leg, both against (blue) and with (red) the grain. The mean penetration depth of the hair tips $h=10 \mathrm{~m}$. The peaks correspond to dynamic force interactions. c) The dependence of the three contact-force components on $h$. d) The 'Bug Rug': an idealized unidirectional superhydrophobic surface based on the form of water-walking arthropod cuticle. . .

6-1 (a) A juvenile Wilson's Phalarope feeding. Note the prey suspended in the droplet trapped in its beak (inset).Photo courtesy of Robert Lewis. (b) Shorebirds employ a variety of foraging strategies and so exhibit large variations in beak size and shape. Here we plot bill length and base width of common shorebirds with straight bills. . . . . . . . . . . . . .

6-2 Fluid drop in a horizontal beak. (a) Schematic of a bird beak with a fluid drop trapped between upper and lower mandibles. (b) A completely wetting drop of silicone oil $\left(\theta_{1}=\theta_{2}=0\right.$, dynamic viscosity $\left.\eta=0.05 \mathrm{~kg} \mathrm{~m}^{-1} \mathrm{~s}^{-2}\right)$ selfpropels towards the apex of a mechanical bird beak with a constant opening angle $\alpha=3.4^{\circ}$ and uniform width $1 \mathrm{~mm}$. Scale bar $2 \mathrm{~mm}$. (c) Plot of drop front position versus time for silicone oil $\left(\eta=0.01 \mathrm{~kg} \mathrm{~m}^{-1} \mathrm{~s}^{-2}\right)$ for three opening angles $\alpha_{1}=1.9^{\circ}, \alpha_{2}=2.8^{\circ}$ and $\alpha_{3}=4.2^{\circ}$, where $\left(L_{b}-x\right)(\mathrm{mm})$ represents the distance travelled by the drop from the beak tip. . . . . . .

6-3 The capillary ratchet. (a) Time sequence illustrating the water droplet transport generated by an opening and closing cycle of the mechanical beak. In the closing cycle, the leading contact line proceeds towards the mouth; in the opening cycle, the trailing contact line recedes towards the mouth. The result is net drop transport towards the mouth. Scale bar, $2 \mathrm{~mm}$. (b) Plot of the associated motion of the leading (red) and trailing (green) contact lines generated by varying the opening angle $\alpha$ over three cycles. $d(\mathrm{~mm})$ represents the distance from the beak tip to the contact line. See also Supplementary Movie S2. . . . . . . . . . . . . . . . . . . . . . . 130 
6-4 (a) A schematic illustration of droplet dynamics in an oscillating bird beak. The drop is pinned for region $\theta_{a}>\theta_{1}>\theta_{2}>\theta_{r}$, marked by the red line. As the beak is closed progressively, first the leading (A) then the leading and trailing (B) contact lines advance. As the beak is opened, first the trailing (B) then the trailing and leading (A) contact lines retreat. Ultimately, the drop breaks when $\alpha>\alpha_{\text {break }}$. The ratcheting regime is indicated in green and the optimal ratchet by the red arrows. (b) Regime diagram for droplet transport in an oscillating mechanical bird beak illustrates the dependence of the system behavior on the minimum and maximum opening angles, respectively, $\alpha_{\text {close }}$ and $\alpha_{\text {open }}>\alpha_{\text {close }}$. The drop volume was fixed at $1.5 \mu \mathrm{l}$. For $\alpha_{\text {open }}>\alpha_{\text {break }}$, the drop breaks, while for $\alpha_{\text {close }}<\alpha_{\text {closemin }}$, the drop fills and spills from the beak. The numbers denote the number of cycles required to transport the drop from the beak tip to the mouth in the ratcheting regime. The optimal capillary ratchet transports the drop in three cycles. . . . . . .

6-5 A droplet trapped in a wedge (a) Experimental (red squares) and 3D numerical (black circles) evaluation via Surface Evolver of the critical angle $\alpha_{c}$ at which a drop of volume $\Omega$ breaks. (b) Quasi-static evolution of the interface of a partially wetting drop trapped between two non-parallel plates with increasing wedge angle $\alpha$. The red curve depicts the numerical simulation derived by minimizing surface energy, which closely matches the observed interface shape. (c) Numerically deduced variation of contact angles $\theta_{1}$ (black curve) and $\theta_{2}$ (green curve) with beak opening angle $\alpha$. Advancing and retreating angles, respectively $\theta_{a}$ and $\theta_{r}$, are marked with dotted red lines. The drop remains pinned provided $\theta_{R}<\theta_{2}<\theta_{1}<\theta_{A}$, corresponding to the shaded region. (d) Variation of contact angle along the perimeter of an asymmetric liquid bridge for varying droplet volumes $\Omega_{1}=2 \mu l, \Omega_{2}=1.5 \mu l, \Omega_{3}=1 \mu l$. The model beak has a width $W=1 \mathrm{~mm}$, length $L_{b}=1.5 \mathrm{~cm}$ and fixed opening

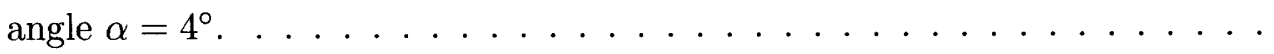

7-1 Static and dynamic generation of combinatorial libraries (a) Cross-wire microchannels separated by a diffusion barrier is utilized to build a static combinatorial library.(b) A continuous-phase combinatorial generation of reagents in a microfluidic manifold. . . . . . . . . . . . . . . . .

7-2 Manual synthesis of combinatorial liposome library with liposomes fused via micro-manipulators under manual control. . . . . . . . . . . .

7-3 Schematic of an all-fluidic droplet combinatorial processor with 8 input lines, 4 output lines and no control lines for continuous production of a library of $4 \times 4$ compounds with integrated modules for logical routing, fusion and

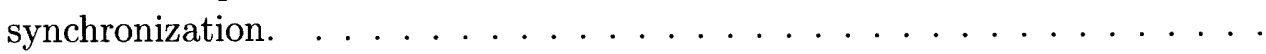

7-4 (a) Schematic layout for a random access chemical memory for storage and retrieval of sub-nanoliter fluid droplets depicting multiplexors for control and flip-flop array for storage. (b) Microphotograph of a one-bit flip-flop. (c) Microphotograph of AND/NOT logic gate. . . . . . . . . .

7-5 Frequency controlled stimulation of surface bound substrates using droplets and bubbles. (a,b) Cell and vesicle micro-arrays bound to the chip surface. 
7-6 Mechanics of odorant transport from bubbles to olfactory receptors on the chip surface. The thin surrounding liquid film effectively acts as an artificial mucus (see inset with odorant molecules depicted in color). . . . . . . . . .

7-7 Microfluidic lock-in amplifier: phase sensitive detection of a sample via tunable frequency intake. . . . . . . . . . . . . . . . . . . . 159

7-8 Sub-nanoliter chemistry, defined as reactions performed in micron-scale droplets in microfluidic systems. The Image depicts methods including trapping, biomolecular amplification, optimization, storage and detection of biomolecules. The ability to generate and manipulate sub-nanoliter droplets in a confined geometries coupled with ultra-senstivie inline-detection schemes enable the implementation of high-throughput assays for biological and chemical analysis. . . . . . . . . . . . . . . . . . . 161

7-9 Biological machines employing fluidic means of locomotion and control. . . 163

7-10 Bubble display. (a) Schematic of programmed generation of drops and bubbles in a serpentine channel for a microfluidic display element. (b) Controlled generation of mercury droplets in a fluidic channel for programmable $\mathrm{RF}$ components. . . . . . . . . . . . . . . . .

Portable mechanical pumps currently used for controlling amount of delivered drug to the body. (a)Early prototype of insulin pump that delivered glucagon (Image courtesy Whitehall Lab, Indiana 1963). (b) Currently used insulin pumps with stepper motor based mechanical means for drug metering. (c) Next generation pump designs for drug delivery manufactured via microfabrication techniques. Image courtesy Debiotech Inc.(www.debiotech.com)

7-12 Programmable microfluidic assembly and replication of droplet strings. Bubble logic devices, with programmable control of hydrodynamic flow fields provide a platform to fabricate and replicate string like microscopic chains.

7-13 Next steps for development of bubble logic. The three efforts are focused on tool development, exploring new physics in these devices and applying the principles learned to various domains of biology, chemistry and material science. . . . . . . . . . . . . . . . . . 170 


\section{Chapter 1}

\section{Introduction}

\subsection{Information is Physical}

Conventionally, computation is tied to manipulation of information. From a physical standpoint, computation can also be understood based on physical constraints due to laws of physics and what implications it has on the physical laws itself. John Wheeler [33] proposed a connection between physics and computation in his theory of "it from bit"- proposing that all reality is derived from information. Wheeler at length discusses - what can computation and information predict about physics? Can physical laws be derived from computation and information theory - i.e. get "it" (physics) from "bit" (computation).

Rolf Landauer [21], who popularized the phrase "Information is physical," looked at how laws of physics constraint the ultimate limits of computation in physically realizable computers. Rolf argues that information is always represented by physical objects: be it photons, electrons, or chalk marks on a blackboard. In the 60's, Landauer [20], while working at IBM, established a theoretical lower bound on entropic energy cost of erasure of one bit as $k \ln (2)$, where $k$ is the Boltzmann constant. Thus Landauer's principle, for the first time, related Shannon entropy to thermal entropy in a logic operation. Soon after, in seminal work by Charlie Bennett $[4,5]$ logical reversibility of computation was established. Thus physical 
representation of information and hence computation is bounded by laws of physics. Hence computation is always physical, utilizing physical degrees of freedom.

With the viewpoint that computation is a physical process, new physical computers are under development targeting to reach the fundamental limits of computation. These insights into physicality of computation, simple in retrospect, led to a better understanding of physics of information processing and thus more efficient implementation of computation [1.9]. However, even though "information is physical" is appreciated by the device physics community for designing ever smaller computational elements, it has primarily remained a theoretical tool to probe the ultimate limits of computational systems.

In this thesis, I propose a new paradigm in computation where "physical bits" can be used to simultaneously manipulate both materials and information in a physical system. Depending on the choice of the bit, a desired material payload can be attached to individual bits. Thus during the course of information processing or computation, programmable material manipulation is simultaneously achieved - requiring no external control. Such a literal translation of "information is physical" has deep implications in the field of microfluidics, material science, chemistry, programmable matter and digital fabrication.

With this insight, I experimentally implement a physical system that allows for integrated material and information processing. "Microfluidic Bubble Logic" [25] is a computational scheme that is capable of programmable, sub-nanoliter volume, on-chip, universal fluidic computation and control in a physical fluid dynamic system. Merging ideas from digital logic and fluid dynamics, I present a two-phase all-fluidic logic family implementing universal computation at low Reynolds and capillary numbers employing only Newtonian fluids.

The above described scheme has similarities in bit representation to lattice gas models of computation, most commonly referred as billiard ball computation (see figure 1-1) by mathematical physicists. Billiard ball model of computation was proposed by Fredkin and Toffoli [10] in a seminal paper in 1982 based on there ideas on conservative logic schemes. The scheme provided a platform for exploring non-dissipative computations via perfectly elastic collisions of balls in an unstructured environment. The work expanded in numerous branches 


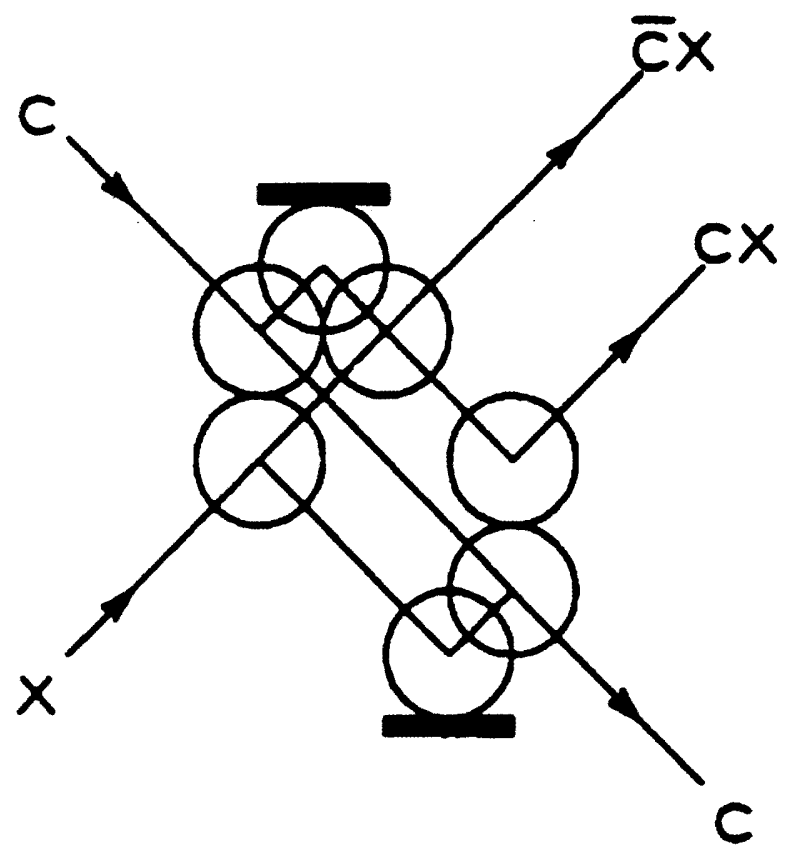

Figure 1-1: Reversible logic scheme implemented as collision of hard balls, where presence or absence of a ball represents 0 or 1 . Reflecting mirrors and geometrical arrangements govern the interaction patterns for the balls. Image from [10]

giving rise to electronic implementations of reversible computation, cellular-automata models of computation and numerous generic models for collision based computing [1]. However, these ideas were either only theoretical framework for studying computation or implemented purely in electronic systems.

\subsection{Manipulating materials at small length scales}

In the era of systems biology, automation of measurement processes is enabling a deeper understanding of bio-molecular processes in living systems. High-throughput data collection lies at the heart of such an effort with precise, quantitative measurement of biological processes in a large variety of conditions. Furthermore, with the ability to conduct high throughput experiments in combinatorial material science and chemistry, a new set of designer materials can now be explored - anywhere from search for new materials for solar energy to drug targets for human diseases. 


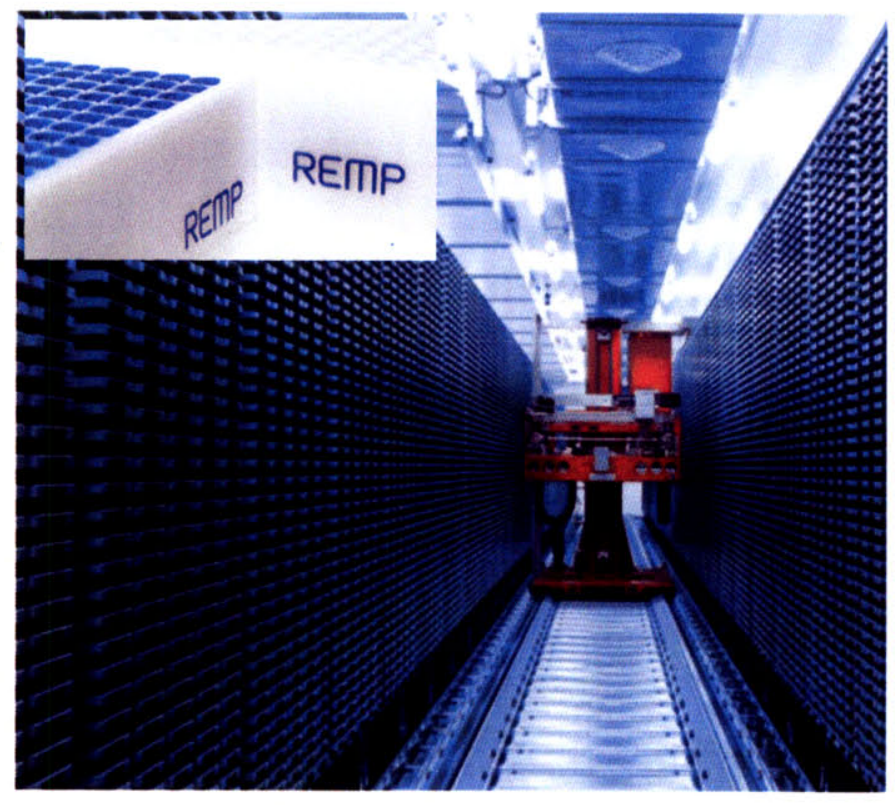

Figure 1-2: Conventional liquid handling robots are large, bulky, slow, costly to operate and require micro-liters of expensive reagents for individual screening condition; thus making it financially non-viable for screening millions of conditions or performing complex assays in a few seconds. Inset depicts a micro-titer plate for chemical storage (Image from REMP product catalog).

Robotic automation tools are still the primary means of liquid handling in most highthroughput experiments in the pharmaceutical, chemical and micro-electronics industry. Robotic liquid handling; with the ability to take a set of commands and sequentially execute in a deterministic manner, provides a basis set of most commonly used tools in genomics, proteomics and combinatorial material science research. Primarily, these tools are programmed to perform volume metering, dispensing and mixing sequences for chemical reagents. The most common limiting factors to be considered while setting up a high throughput screen are cost of reagents and time it takes to perform a screening operation. For conventional, off the shelf robotic tools, a screen with 100,000 compounds with 10 concentration variations typically takes several liters of reagents and takes several months to complete, running $24 / 7$. This is a bottleneck for setting up large scale screens due to significant cost of reagents for biological assays. Furthermore, the equipment for a programmable liquid handling robot is also bulky and expensive. Thus only big laboratories can afford to run such a setup for long periods of time. 
Microfluidics- automated liquid handling in integrated micro-chips; brings this capability within reach of experimentalists and biologists. Operating at ever reducing length scales, microfluidics provides the benefit of shrinking sizes, faster reactions and optimized synthesis for chemistry, biology and material sciences. A new revolution of the scale of personal computing will be unveiled in genetics, pharmaceuticals, analytical chemistry, systems biology and healthcare diagnostics, if microfluidic technologies hold to the promise they offer. Dramatic improvements in our capability to precisely manipulate extremely small volumes of fluids (nano-liters to femto-liters) is already shaping the future of this relatively nascent field. Explosive progress is already being made, both in terms of fundamental technology development for large-scale integration [29] of microfluidic functionalities on-chip and appropriate chemistry for performing biological [35] and chemical functionalities on-chip. A large number of breakthroughs in systems biology $[16,2,17,26]$ can already be credited to this fundamental advancement in fluid manipulation technology. In-spite of the current progress, microfluidic technologies still rely on macro-scale external control elements hindering very large-scale integration and transition of microfluidic devices from research laboratories to field-deployment of low-cost portable instruments.

\subsection{All-fluidic logic and control}

This thesis presents an approach based on micro-fluidic computing for the design of a scalable fluidic processors enabling internal flow control with no-moving parts.

\subsubsection{High Reynolds number}

Fluidic computing as a field has existed since the 60's when fluidic technologies based on inertial non-linear effects like "Coanda effect", "jet-interaction" etc. operating at high Reynolds numbers competed in performance and applications with electronic counter parts. The idea of using fluidic computing elements for information processing was later rejected in the late 70's owing to slow performance and a fundamental limitation perceived in shrink- 

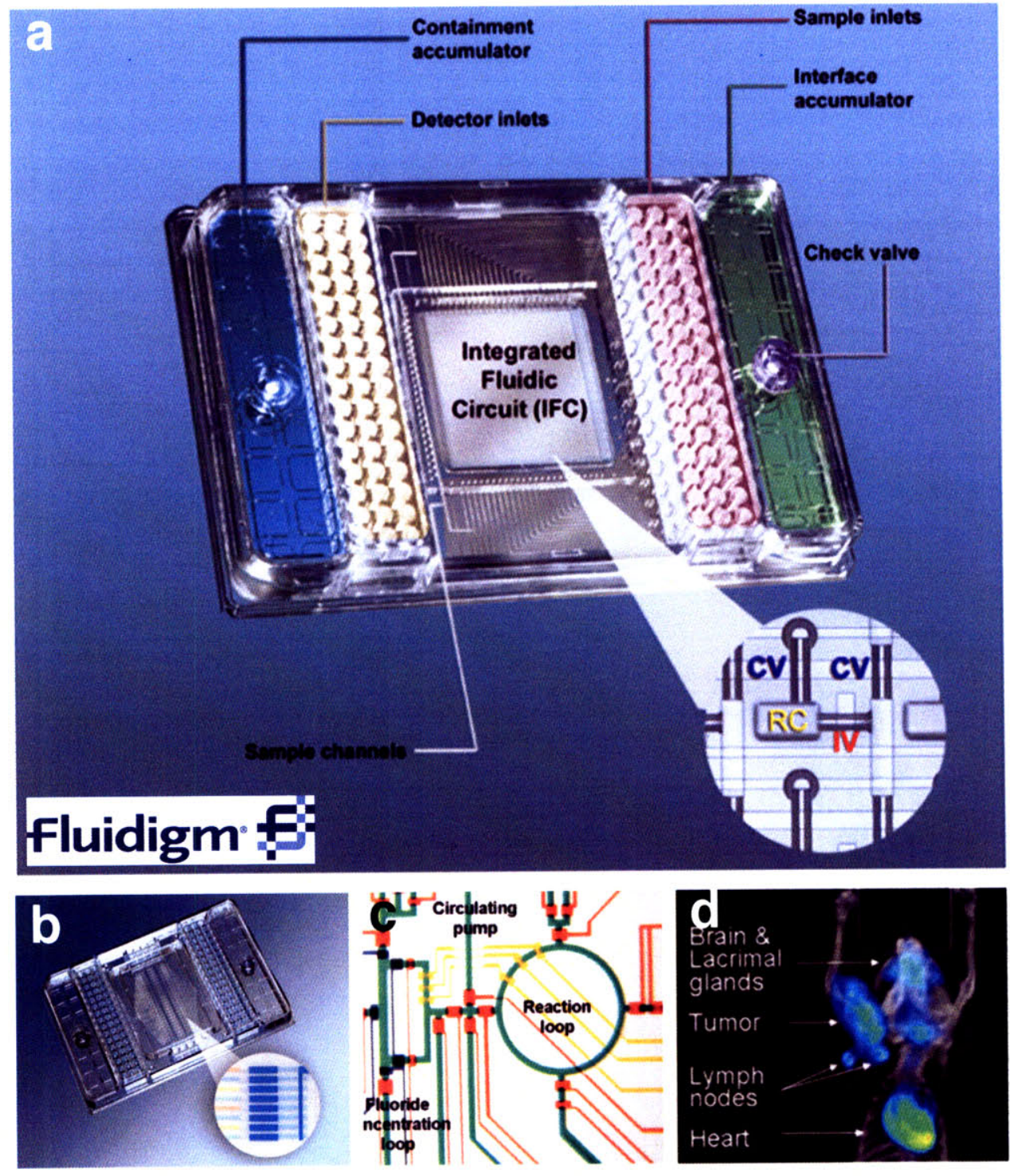

Figure 1-3: Integrated fluidic circuits (IFC) employing "Nano-flex" elastomeric valves. (a) Photograph of a $48 \times 48$ dynamic array micro-chip using elastomeric valves, commercially available from Fluidigm (www.fluidigm.com). Image of from [28]. (b) Photograph of a protein crystallization chip (TOPAZ, fluidigm) with clear elastomeric IFC integrated in a carrier for liquid delivery. Parallel lines represent reagent inlet into diffusion chambers. Image from Fluidigm product catalog. (c,d) Microchip with reaction loop, integrated valves and pumps for on-chip synthesis of radio-labeled fluorine as cancer probe. Image from [22] 
ing the device sizes further (since inertial effects are no longer dominant at low Reynolds numbers) without drastically affecting the device physics and hence poor performance.

Reynolds number is defined as ratio of inertial and viscous forces in a fluid medium i.e. $R e=\rho u L / \eta$ where $\rho$ is the fluid density, $L$ is the relevant length scale, $u$ is velocity of fluid element and $\eta$ is dynamic viscosity. Inertia based fluid logic elements were designed for flow regimes where $R e>1000$ (turbulent flow is usually defined as $R e>2000$ ). For conventional microchannels with $L \sim 100 \mu \mathrm{m}, u \sim 0.1 \mathrm{~cm} / \mathrm{sec}, \eta \sim 0.01 \mathrm{gm} / \mathrm{cm} \mathrm{sec}$ and $\rho \sim$ $1 \mathrm{gm} / \mathrm{cm}^{3}$ for water, typical Reynolds number in microfluidic devices is $R e \sim 0.1$. Thus for reaching a $R e>1000$, a flow velocity of $u \sim 10 \mathrm{~m} / \mathrm{sec}$ is required. Such high velocities are extremely difficult to achieve in micro-scale geometries.

Several families of logic circuits utilizing nonlinearities in physical fluid dynamics have been reported in the past. Fluidic logic from the 1960's [8,3] was dependent on the inertial properties of fluid flow thus requiring high Reynolds number flow. Such fluidic gates were used to build a trajectory controller, an all-fluidic display, non-destructive memory and a simple computer [3]. All-fluidic logic [7, 8, 3] as a field was launched in the 1960's with the realization that non-linear inertial interactions of air-jets could be used to enable control of pneumatic machinery. This novel integration of actuation and control which is extremely desirable for buildign complex systems is otherwise extremely hard to achieve. Inertia-based fluidic logic was successfully deployed in hazardous settings where microelectronics still performed unreliably and the "computational count" required was low. A major drawback of this technology, widely called "fluidics" was its dependence on high-Reynolds number flows. Since the devices could not be shrunk in sizes, without involving super-sonic velocities [34], the growth in the field was crippled with the advent of new micro-electronics based control technologies.

\subsubsection{Low Reynolds number}

In this work, I show that this dependence on high-Reynolds number is unnecessary. NavierStokes equations describing fluid flow, are highly non-linear in all flow regimes. In this 

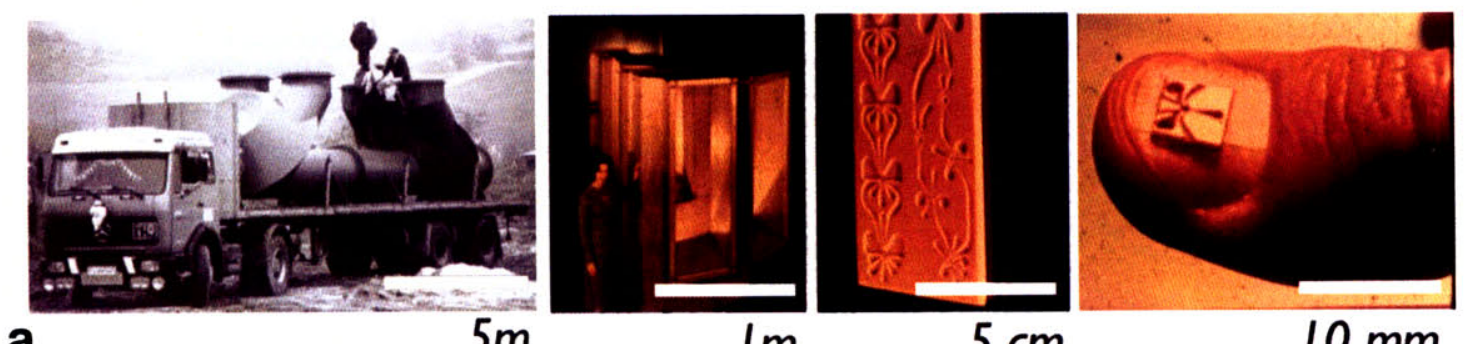

a

$5 m$

\section{Im}

$5 \mathrm{~cm}$

$10 \mathrm{~mm}$
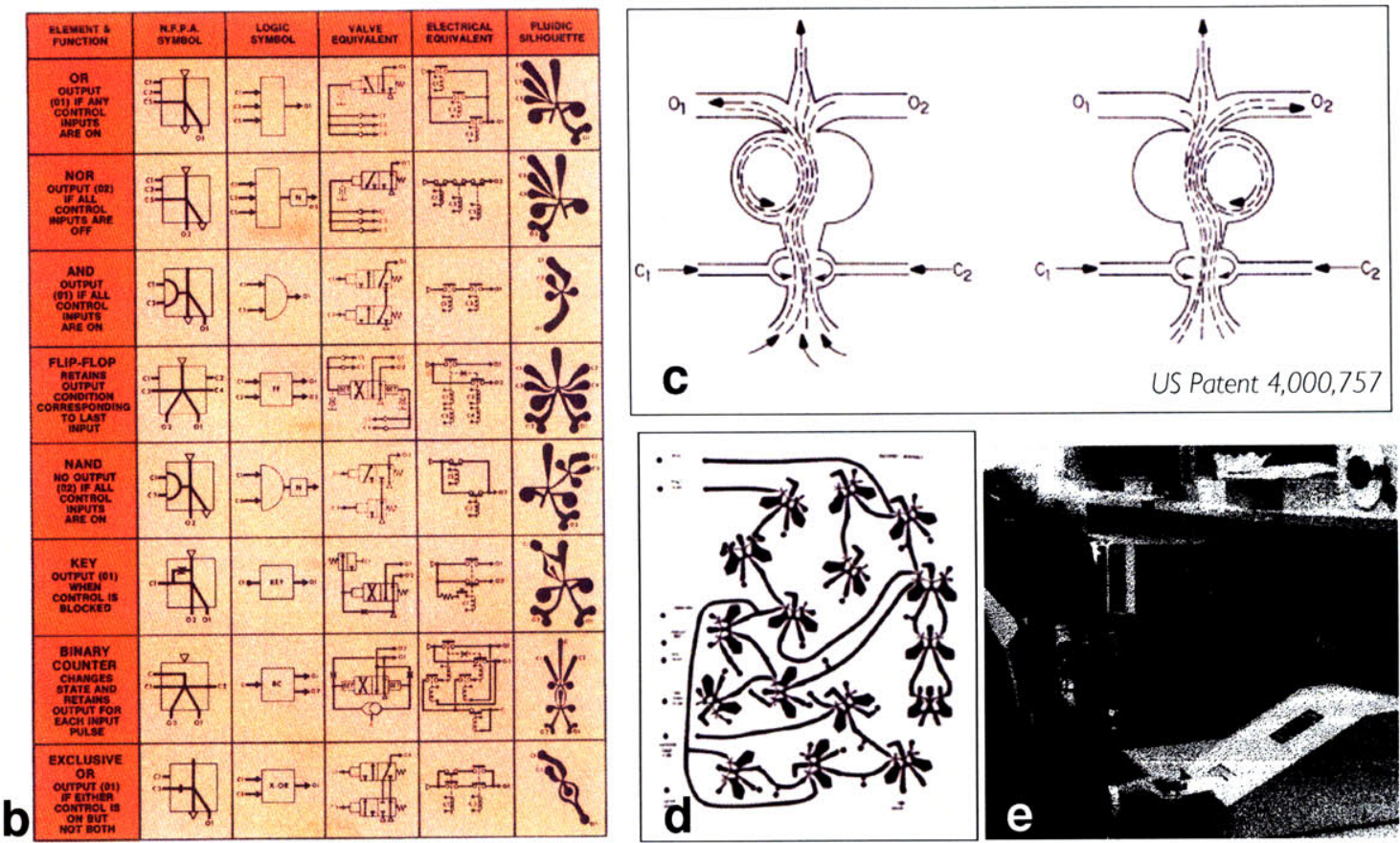

C

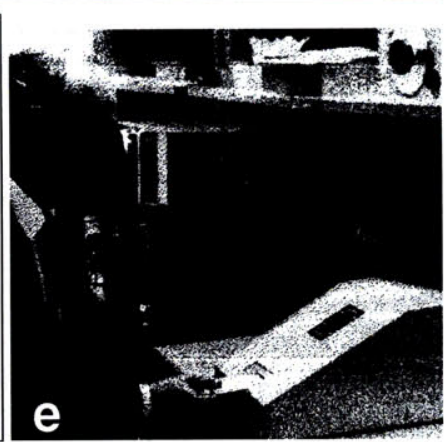

Figure 1-4: (a) High Reynolds number fluidic logic gates from the 06's with varying size from meter scales vortex valves to milli-metric scale proportional amplifier. (b) Schematic description of fluidic logic gate with equivalent electrical analogs depicted. (c) Vortex ring based jet-amplifier with output jet stream controlled by inlet control lines. (d,e) Integrated fluidic logic gates with a schematic integrated control circuit and an all-fluidic display from UNIVAC [7]. 
work, instead of using inertial forces as primary non-linear phenomena, I demonstrate that interfacial and viscous forces, which are dominant at low-Reynolds number can be exploited to built a universal family of logic gates. This allows us to shrink device geometries to micron length scales with favorable scaling laws towards further miniaturization. Miniaturization also allows us to handle extremely small sample fluid volumes (nano to femto-liters) which is desirable for any successful microfluidic technology. With miniaturization, which was necessary for higher operating speeds and integration, it was impossible to maintain high Reynolds number flow in microscopic geometries. Previously, fluids with polymer additives have been used to implement a constant flow source and a bistable gate $[14,15]$ but the operation of these devices is dependent on non-Newtonian fluid properties (see figure 15). Change in flow resistance has been used [31] to build Boolean logic in a single-phase Newtonian fluid, but since its input and output representation are not the same these devices could not be cascaded.

Bubble logic [25], based on hydrodynamic bubble-to-bubble interactions provides a scalable universal logic family employing newtonian fluids. A droplet or a bubble traversing in a channel represents a bit. The bit representation for bubble logic is similar to theoretical billiard ball logic [10] which based on the elastic collision of particles, and magnetic bubble memory [6] relying on interactions of magnetic domains in garnet films. These schemes all conserve information because during a logic operation a bit is neither created nor destroyed.

Microfluidics has quickly grown from a research technique to an industry accepted platform technology for small volume liquid handling. Currently, specialized chips are designed and fabricated based on specific applications. Unlike micro-electronics where common modules are put together to build more complex circuits, microfluidics still requires design and synthesis of fluidic elements from scratch for a given application chip. Modularity of individual elements and standardization of parts would shift this trend towards small number of general purpose microfluidic processors. Another motivation for this thesis in developing an all-fluidic logic family and numerous control elements based on the same is to allow modular design of microfluidic circuits. Since the transfer characteristics and operating regimes of individual elements can be specified, a microfluidic engineer should be able to 


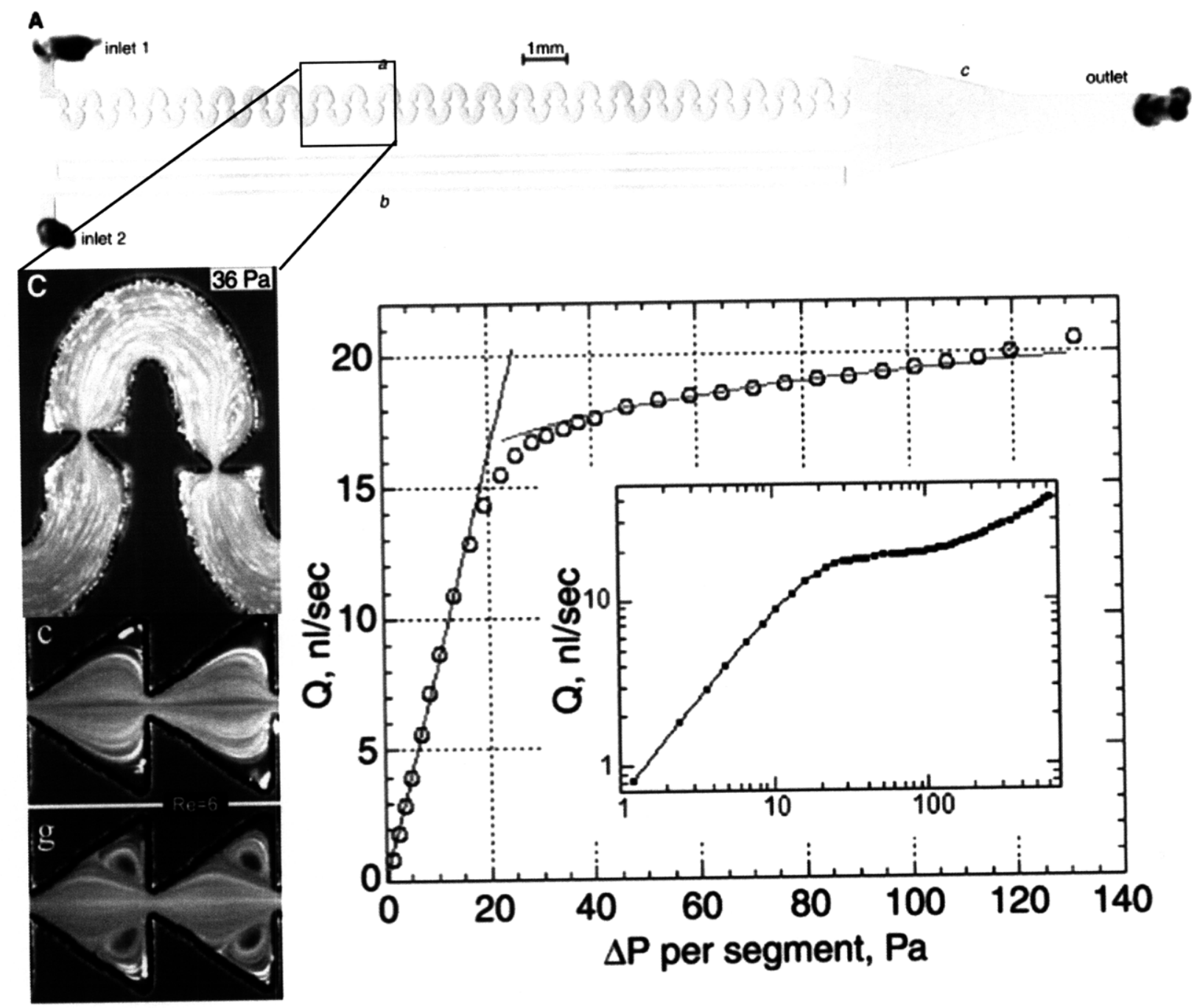

Figure 1-5: Fluidic memory and control elements using non-newtonian fluids [13]. Extension and relaxation of polymer molecules results in asymmetric flow behavior in an expansion and contraction geometry, also depicted by pressure vs. flow-rate characteristic curve for a given channel geometry. Image from [13]. 

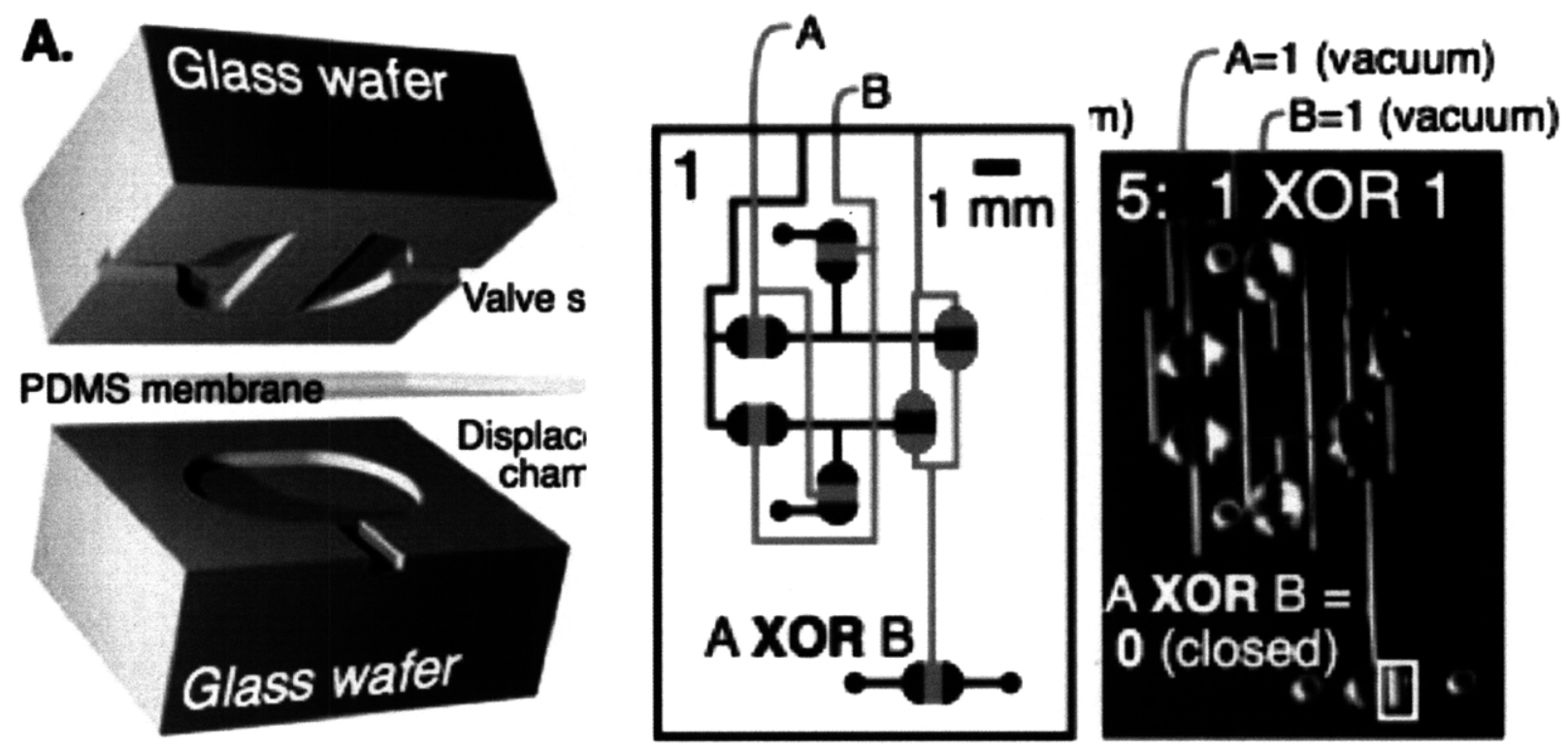

PDMS membrane

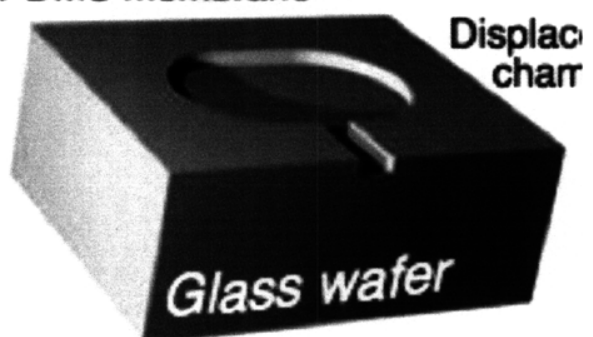

Figure 1-6: Fluidic valves based on flexible membranes sandwiched between glass wafers [18]. The valve can be turned on and off via negative pressure and can be latched in either on and off position. A simple XOR gate formed using six pneumatic valves. Image from $[18]$.

pick components from the parts library to simply build fluidic circuits for a specific experiments. Moreover this approach allows for a general hierarchy of components which can be pre-assembled in this manner.

Fluid dynamics of single-phase flow at low Reynolds number in microchannels is laminar in nature [2], and throughly studied [9]. However interfacial microfluidics, involving fluid-fluid interfaces (for example drops and bubbles) in confined micro-geometries, exhibits complex non-linear flow behaviors due to hydrodynamic interactions and is under an intense phase of development $[30,32,11,12]$. In this thesis, I experimentally demonstrate novel non-linear hydrodynamic interactions in multi-phase flow. Moreover, this thesis presents a systematic approach to designing several microfluidic control elements via hydrodynamic interactions of drops and bubbles in confined geometries. 


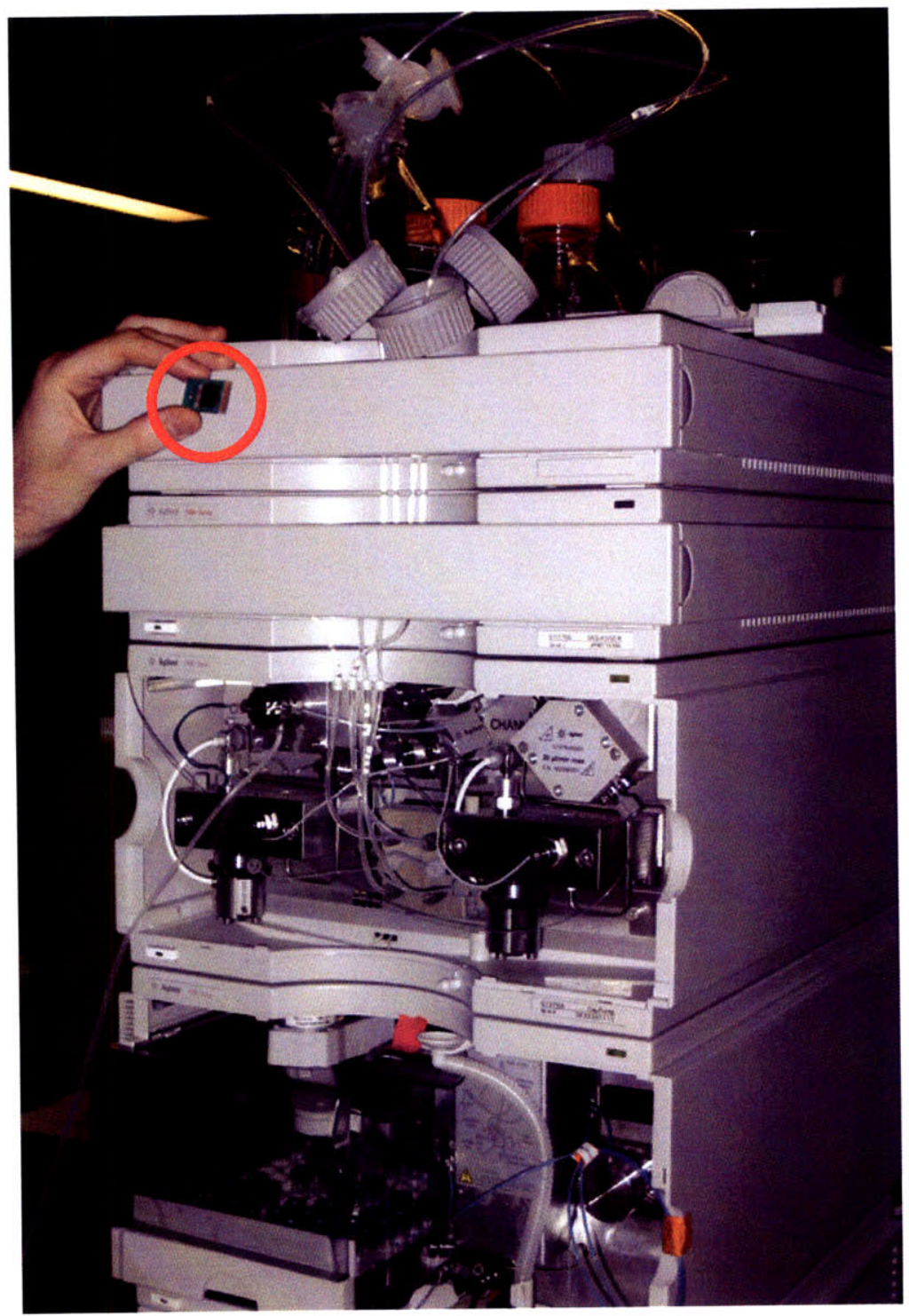

Figure 1-7: Comparison of a chip-scale detection technologies with convention liquid handling equipment required for automated operation of such a device (Image courtesy Will Grover, Manalis Lab, MIT). Usually, large liquid handling and control equipment is required to handle samples on micro-chips, leading to the notion of a "lab around a chip". 


\subsection{Compartmentalization: Droplet based digital materials}

In an information processing paradigm, our choice of bits is based on several factors. In implementation of a classical computer, the choice is guided by several factors like switching speeds, energy consumption, physical transport limitations and size of the bits used [19]. For bits to be able to manipulate both materials and information, another requirement is to be able to attach discrete packets of a large variety of materials without drastically changing the nature of individual bits itself. For the system described in this thesis, I choose discrete packets of fluid (drops and bubbles) embedded in a continuos phase individual bits (see figure 1-9). Due to mass conservation, number of fluidic bits entering a logic gate needs to be equal to number of bits exiting the gate geometry. This allows us to implement conservative logic family, where multiple Boolean logic operations can be computed at the same instance of time. This paradigm also makes it possible to implement a reversible logic scheme, where if the direction of flow is reversed; the initial starting inputs at any point of time during a computation can be retrieved. Moreover if required, individual drops and bubbles can be fused or divided into multiple entities allowing numerous chemical steps like reactions, dilutions and metering possible. This allows us to implement the basic modules required for generic biological and chemical processing.

Advances in microfluidic experimental techniques for generation of mono-disperse subnanoliter volume emulsions has led to an explosive growth of a new class of bio-chemical materials. Loosely termed as nano-liter droplet based materials, a very large variety of droplet emulsions with complex structures (for e.g. multi-emulsions) can be synthesized employing microfluidic based emulsion technologies [12]. Controlled generation frequency $(10 \mathrm{~Hz}-1 \mathrm{Khz})[32]$ and droplet volume (femto to nano liters) [11] has been demonstrated. My work also introduces a new technique for on-demand generation of drops and bubbles [25]. Figure 1-9 depicts a small array of materials (organized as living vs. non-living and natural vs. synthetic) that can be trapped in droplet based emulsion for further processing. A vast variety of novel surfactant chemistries [36] are also under development suitable for numerous biological assays. Thus microfluidic emulsion technologies $[30,32,11,12]$ provide 


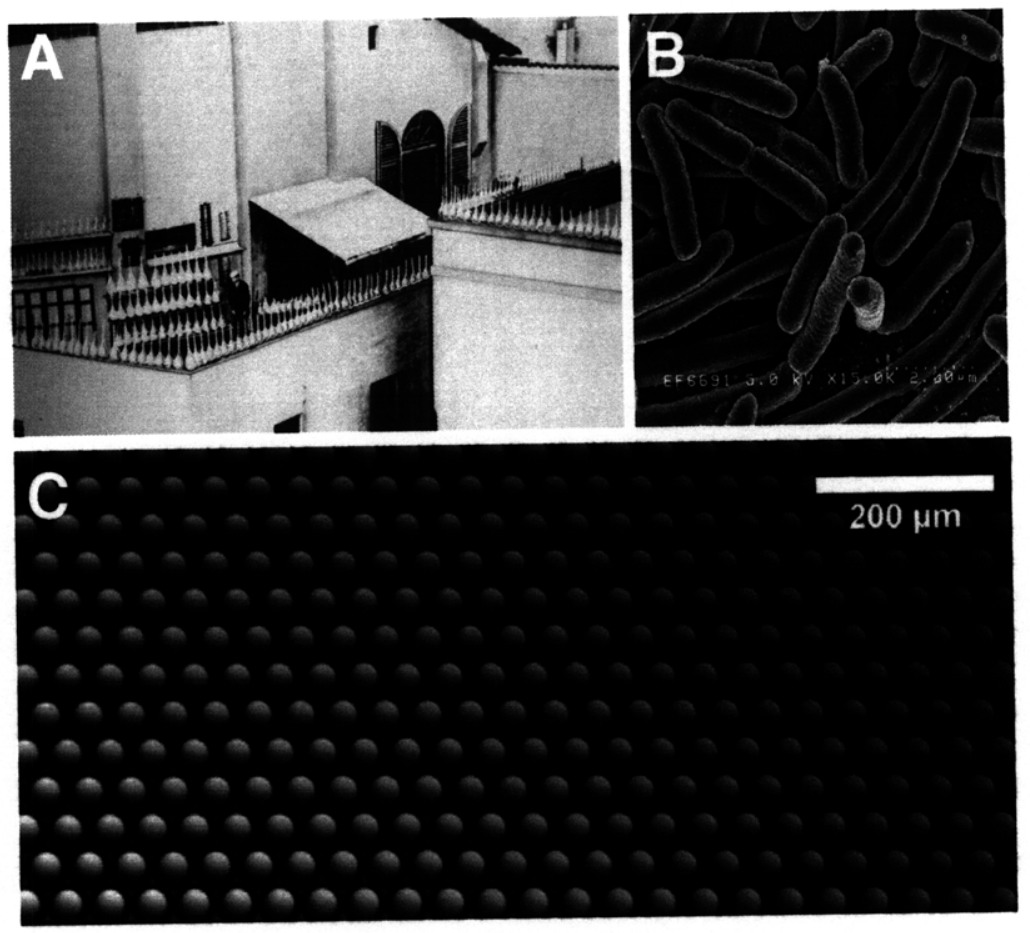

Figure 1-8: Compartmentalization in natural and artificial systems. (A) Giacomo Ciamician (1857-1922), father of green chemistry, on the roof of the Chemical Institute in Bologna among the flasks he is exposing to solar light running experiments in combinatorial chemistry. (B) SEM images of E-coli, roughly a $1 \mu \mathrm{m}$ in length serve as individual biological compartments. (C) Mono-disperse fluid droplets, $40 \mu \mathrm{m}$ in size generated using microfluidic emulsion techniques, can act as artificial cells capable of performing chemical reactions in confined volumes. 


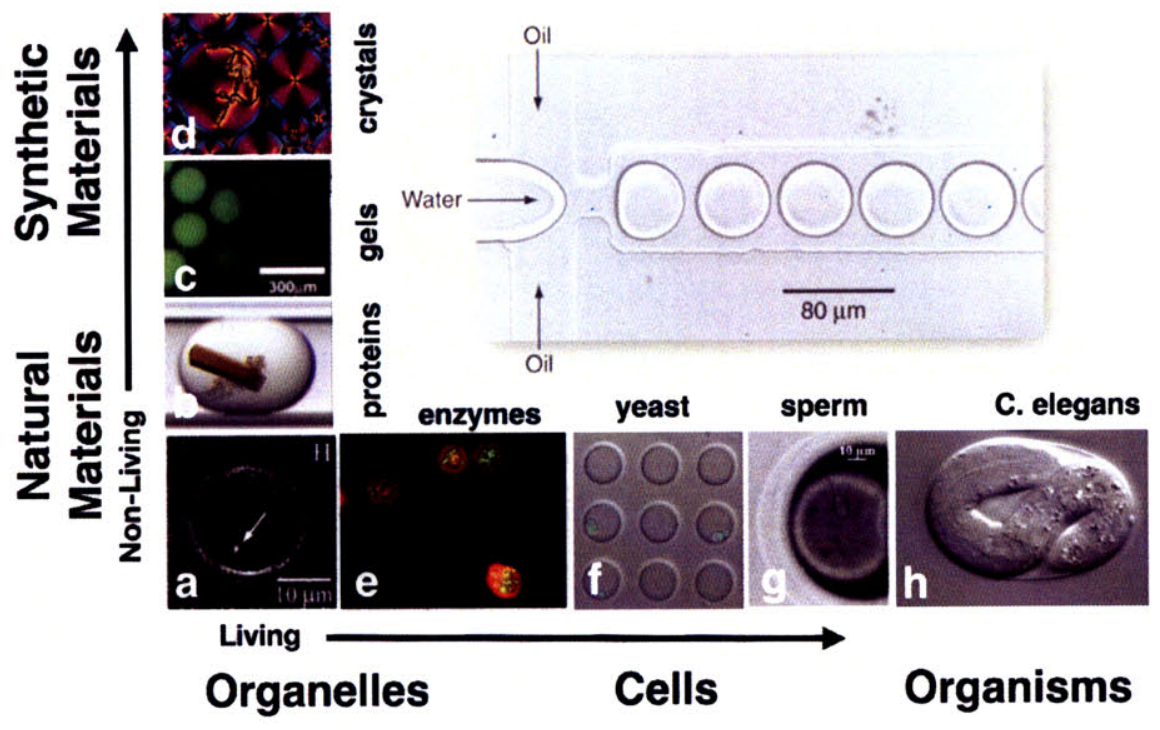

Figure 1-9: Encapsulation of natural and synthetic materials in compartmentalized droplets. A large array of nano-bio materials can be encapsulated using the prescribed scheme including bio-molecules, single organelles, single cells, whole organisms, protein crystals, liquid crystals and so on.

a means to generate a new class of materials (which I call digital because of discrete nature of the same) which was not possible before.

With the advent of nano-liter droplet based materials, a scalable means to manipulate, store, organize and retrieve these droplets is required (fig. 1-9). Microfluidic computing where drops and bubbles traversing in microfluidic channels represent bits of information can be used as a paradigm for scalable internal control mechanism for handling this new class of materials. Such a technology would invariably make a large impact on a vast number of fields including single cell analysis and assays, combinatorial chemistry and droplet based sub-nanoliter chemistry.

\subsection{Nanoliter chemistry}

A clear trend has been established over the last decade leading to ever-shrinking reaction volumes for bio-chemical reactions. Starting with conventional test tube based manipulation 
of milli-liter volumes, common in all chemistry labs, to micro-liter fluid manipulation using manual and automated pipetting systems which allow for 100 reaction wells in plastic micro-well plates. Micro-well plates provide spatial separation of micro-liter volume wells in a spatially organized format, that easily renders itself to robotic automation. Furthermore, evaporation or dilution can be achieved via an open port. Due to already established infrastructure to handle micro-well plates, several templates have been created that connect to micro-well plate and automate several aspects of reagent filling and screening via fluidic manifolds underlying the wells.

A significant step towards miniaturization of biological sample analysis came with development of techniques to fabricate micro-arrays consisting of liquid droplets, $100 \mu \mathrm{m}$ or smaller in size, on solid substrates. Since a large number of samples could be spotted on solid substrates and imaged simultaneously, a large number of genetic and protein screens could be implemented in this format. A drawback of such an implementation is that once the reaction mixture has been created and spotted on the surface, no reagents can be added or extracted from the mixture.

As described previously, microfluidic manipulation of fluid samples brought a revolutionary change in how chemical and biological reagents can be handled in automated or semiautomated manner in micro-fabricated channel networks. Two different paradigms in sample handling in engineered systems have emerged over the last decade, single-phase and multi-phase fluid handling. Reaction volumes in single-phase systems is defined by the volume of sealed channels. These reaction volumes can be dynamically manipulated by opening and closing integrated valves, leading to a high customizable platform. Multi-phase or droplet microfluidic systems define reaction volumes as the reagents captured in individual droplets. Such a discrete scheme allows for manipulation of large number of unique samples in the same micro-fabricated channel geometries without any cross-contamination. Reaction volume for droplet based microfluidic systems vary from a few nano-liters to several femto-liters. The capability to reach volumes of the range of femto-liters (typically found in single cells) allows us to study reaction conditions found in natural counterparts or very dilute systems (single cell or single molecule assays). 
In this thesis, I primarily focus on micro-droplets (defined as $100 \mu \mathrm{m}$ to $1 \mu \mathrm{m}$ diamter droplets) as a platform for nanoliter chemistry. A large number of chemistries have been ported to such a platform including multi-step protein crystallization assays [23], gradient screening for crystallization conditions [37], polymerase chain reactions [24], study of millisecond enzyme kinetics [27], directed evolution of enzymes and so on. This provides a very broad range of reaction conditions that can be satisfied in micro-fabricated systems employing individual droplets as discrete reactors. Furthermore, large number of droplets can be created in micro-scale geometries at high-throughput. Thus such a platform has the capability to screen for a large space of reaction chemistry and simple gradient screens have already been developed for the same.

\subsection{Bio-inspired Design}

Microfluidics as a field has gained attention in the last 20 years as a field in chemical, mechanical and bioengineering. Behavior of fluids at small length scales has been a subject matter of intense studies during this time, leading to a better understanding of how complex fluids behave in confined geometries. Furthermore, principles of fluid physics at small length scale provide new physical mechanisms that can be exploited in microfluidic device design.

Biological systems, on the other hand, have extensively exploited and employed microfluidic systems in biological design. For example, blood capillaries, lymph nodes and various transport channels in human body employ channel geometries of micrometer length scales to achieve directed transport and control of chemicals in the body. Plants employ xylem and phloem network to transport materials and water from roots to leaves and vice versa. Lungs employ micro-capillaries to transport oxygen from the inhaled air to oxygenate blood. Carefully and quantitative study of microfluidics in biology would not only lead to better understanding how biological systems function, but further provide design examples that can be exploited in the technological counterparts.

This thesis also describes the discovery of two new physical fluid dynamic mechanisms motivated by a common theme of role of interfacial uid mechanics in biology. Firstly, I 
describe a new superhydrophobic water-repelling surface that has a characteristic of directional anisotropy to uid resistance. The discovery made while studying the integument of water-walking insects, helps rationalize the origin of thrust and hence propulsion of waterwalking insects on a uid interface. Secondly, this thesis uncovers a new physical mechanism for directed droplet transport, which I term Capillary ratchet. Discovered in a class of surface feeding shorebirds, it is the only physical mechanism that is known to exploit contact angle hysteresis for uid transport.

\subsection{Summary of Major Results}

Various original contributions have been made in this thesis. Firstly, I present a means to generate individual nano-liter to femto-liter volume drops on demand via an electro-fluid modulator employing variation in surface tension based on temperature. This allow us to programmatically generate drops and bubbles of reagents on-demand thus encoding signals as an input to the microfluidic chip. I also present a hydrodynamic trap for storage and release of individual drops and bubbles traveling in a flowing stream of continuos phase fluid. This element acts as a fundamental unit of fluidic memory required for storage of drops and bubbles on chip with no external control. Individual flip-flops can be arrayed to build circuit elements like a ripple counter, useful for digitally counting on chip. A conservative universal logic scheme is also presented which provides us the means to route individual drops and bubbles on-chip in a programmable fashion. The logic provides a means to invert,

amplify and manipulate signals encoded in a train of drops and bubbles. As a test circuit, I also present a ring-oscillator built using AND gates and delay lines which allows us to test operating parameters for individual logic gates. For timing restoration, I present a novel fluidic ladder network geometry which is capable of synchronizing drops and bubbles co-flowing in two coupled fluid streams. This allows for error-correction on chip which is necessary for scalability.

In the field of bio-inspired design, I also present a novel valveless means to pump individual droplets via a capillary ratchet. This device employes asymmetry in droplets trapped in a 
wedge geometry to pump fluids via induced vibrations. This mechanism could provide a means to implement a global clock to the system in the future. Furthermore, a new solid surface termed "unidirectional superhydrophobic surfaces" that exhibits isotropic adhesion to fluid contact lines has been discovered in water-walking insects. I also present a method to synthesize an artificial surface exhibiting unidirectional superhydrophobic characteristics.

\subsection{Thesis Structure and Overview}

This thesis is divided into three main sections. Chapter 2, 3, 4 describe my work in implementation of universal logic in purely hydrodynamic systems at low Reynolds numbers. Chapter 2 describes the fundamentals of multi-phase flow at low Reynolds numbers and presents the dynamics of fluid droplets in microfluidic channel networks. Chapter 3 presents experimental and results for implementation of universal logic, bistability, cascadibility and feedback implemented in fluidic circuits. Chapter 4 presents device design principles for microfluidic circuits.

Chapter 5 describes analysis, instrumentation and results for uni-directional hydrophobic surfaces, in context of water-walking creatures. I present the first evidence of existence of anisotropy in resistance to motion of contact lines on hydrophobic surfaces found in waterwalking creatures. I also describe instrumentation custom built for pinning force measurement of contact line traveling on solid surfaces. I conclude by presenting the microscopic source of thrust force generated by water-walking insects.

Chapter 6 presents a new mechanism for synchronous motion of micro-liter droplets via a ratcheting mechanism. Capillary ratchet is the first physical mechanism that exploits the dynamics of contact line motion, particularly contact angle hysteresis to induce droplet motion. I present the work in context of feeding mechanisms utilized by several species of shorebirds.

Chapter 7 describes future work, in context to the three main contributions of this thesis, namely microfluidic bubble logic, uni-directional superhydrophobic surfaces and capillary 
ratchets. I further highlight near-term and long-term applications arising from techniques described in this thesis.

Chapter 8 concludes this thesis providing a vision for role of computer science in physical science and programmability of the physical world. 


\section{Bibliography}

[1] Andrew Adamatzky. Collision-based computing. Springer, 2002.

[2] Frederick K. Balagadde, Lingchong You, Carl L. Hansen, Frances H. Arnold, and Stephen R. Quake. Long-term monitoring of bacteria undergoing programmed population control in a microchemostat. Science, 309(5731):137-140, 2005.

[3] Charles A. Belsterling. Fluidic Systems Design. Wiley Interscience, 1971.

[4] C. H Bennett. Logical reversibility of computation. IBM Journal of Research and Development, 17(6):525-532, 1973.

[5] C. H Bennett. The thermodynamics of computation - a review. IBM Journal of Research and Development, 21(12):905-940, 1982.

[6] Hsu Chang. Magnetic Bubble Logic: Integrated-circuit magnetics for digital storage and processing. IEEE Press, 1975.

[7] R. R. Coleman and H. B. Horton. Univac's reserach in fluidics. In Fluidics Quaterly, 1975.

[8] Arthur Conway. A Guide to Fluidics. Macdonald and Co., 1972.

[9] Anton A. Darhuber and Sandra M. Troian. Principles of microfluidic actuation by modulation of surface stresses. Annu. Rev. Fluid Mech., (37):425-455, 2005.

[10] Edward Fredkin and Tommaso Toffoli. Conservative logic. International Journal of Theoretical Physics, 21:219-253, 1982. 
[11] Piotr Garstecki, Howard A. Stone, and George M. Whitesides. Mechanism for flowrate controlled breakup in confined geometries: A route to monodisperse emulsions. Physical Review Letters, (94), April 2005.

[12] Pitor Garstecki, Irina Gitlin, Willow Diluzio, Geroge M. Whitesides, Eugenia Kumacheva, and Howard A. Stone. Formation of monodisperse bubbles in a microfluidic flow-focusing device. Applied Physics Letters, 85(13):2649-2651, 2004.

[13] Alex Groisman, Markus Enzelberger, and Stephen R. Quake. Microfluidic Memory and Control Devices. Science, 300(5621):955-958, 2003.

[14] Alex Groisman, Markus Enzelberger, and Stephen R. Quake. Microfluidic memory and control devices. Science, 300(5621):955 - 958, 2003.

[15] Alex Groisman and Stephen R. Quake. A microfluidic rectifier: Anisotropic flow resistance at low reynolds numbers. Physics Review Letters, 92(9):094301, 2004.

[16] Jong Wook Hong and Stephen R Quake. Integrated nanoliter systems. Nature Biotechnology, 21(10), 2003.

[17] Jong Wook Hong, Vincent Studer, Giao Hang, W French Anderson, and Stephen R Quake. A nanoliter-scale nucleic acid processor with parallel architecture. Nature Biotechnology, 22, 2004.

[18] Erik C. Jensen, William H. Grover, and Richard A. Mathies. Micropneumatic digital logic structures for integrated microdevice computation and control. Journal of Microelectromechanical Systems, 16(6), 2007.

[19] Robert W. Keyes. What Makes a Good Computer Device? Science, 230(4722):138-144, 1985.

[20] Rolf Landauer. Irreversibility and heat generation in the computing process. IBM Journal of Research and Development, 5:183-191, 1961.

[21] Rolf Landauer. Information is physical. Workshop on Physics of Computation, pages $1-4,1992$. 
[22] Chung-Cheng Lee, Guodong Sui, Arkadij Elizarov, Chengyi Jenny Shu, Young-Shik Shin, Alek N. Dooley, Jiang Huang, Antoine Daridon, Paul Wyatt, David Stout, Hartmuth C. Kolb, Owen N. Witte, Nagichettiar Satyamurthy, James R. Heath, Michael E. Phelps, Stephen R. Quake, and Hsian-Rong Tseng. Multistep Synthesis of a Radiolabeled Imaging Probe Using Integrated Microfluidics. Science, 310(5755):1793-1796, 2005.

[23] Liang Li, Debarshi Mustafi, Qiang Fu, Valentina Tereshko, Delai L. Chen, Joshua D. Tice, and Rustem F. Ismagilov. Nanoliter microfluidic hybrid method for simultaneous screening and optimization validated with crystallization of membrane proteins. PNAS, 103:19243-19248, 2006.

[24] Chabert M, Dorfman KD, de Cremoux P, Roeraade J, and Viovy J-L. Automated microdroplet platform for sample manipulation and polymerase chain reaction. Analytical Chemistry, 78:7722-7728, 2006.

[25] Manu Prakash and Neil Gershenfeld. Microfluidic bubble logic. Science, 315:832-835, 2007.

[26] Maerkl SJ and Quake SR. A systems approach to measuring the binding energy landscapes of transcription factors. Science, 315:233-237, 2007.

[27] Helen Song and Rustem F. Ismagilov. Millisecond kinetics on a microfluidic chip using nanoliters of reagents. J. Am. Chem. Soc., 125:14613-14619, 2003.

[28] Sandra L. Spurgeon, Robert C. Jones, and Ramesh Ramakrishnan. High throughput gene expression measurement with real time pcr in a microfluidic dynamic array. PLoS ONE, 3(2):e1662, Feb 2008.

[29] Todd Thorsen, Sebastian J. Maerkl, and Stephen Quake. Microfluidic large-scale integration. Science, 298(5593):580-584, 2002.

[30] Todd Thorsen, Richard W. Roberts, Frances H. Arnold, and Stephen R. Quake. Dynamic pattern formation in a vesicle-generating microfluidic device. Physics Review Letters, 86(18):4163-4166, April 2001. 
[31] Tor Vestad, David W.M. Marr, and Toshinori Munakata. Flow resistance for microfluidic logic operations. Applied Physics letters, 84:5074-5075, 2004.

[32] Dmitri V. Vezenov, Bran T. Mayers, Daniel B. Wolfe, and George M. Whitesides. Integrated fluorescent light source for optofluic applications. Applied Physics Letters, 86(4), January 2005.

[33] John A. Wheeler. Information, physics, quantum: The search for links. Complexity, Entropy, and the Physics of Information, 5, 1990.

[34] Jay N. Zemel. Behaviour of microfluidic amplifiers. Sensors and Actuators, 1996.

[35] Bo Zheng, L. Spencer Roach, and Rustem F. Ismagilov. Screening of protein crystallization conditions on a microfluidic chip using nanoliter-size droplets. Journal of American Chemical Society, (125):11170- 11171, August 2003.

[36] Bo Zheng, L. Spencer Roach, and Rustem F. Ismagilov. Screening of protein crystallization conditions on a microfluidic chip using nanoliter-size droplets. Journal of American Chemical Society, 125(37):11170- 11171, August 2003.

[37] Bo Zheng, Joshua D. Tice, and Rustem F. Ismagilov. Formation of droplets of alternating composition in microfluidic channels and applications to indexing of concentrations in droplet-based assays. Analytical Chemistry, 76:4977-4982, 2004. 


\section{Chapter 2}

\section{Microfluidic Droplet Networks}

Complex fluid channel networks are ubiquitous in animate and in-animate natural systems. From micro-capillary networks in circulatory systems to porous structure in rocks, examples of intricate connection geometries exist in natural and artificial systems. Pattern formation arising from complex hydrodynamic interactions of flow of multi-phase fluids in such a network topologies is commonly observed [13]. In this chapter we review the principles of multi-phase flow in channel networks, and highlight the use of reduced order models for understanding flow behavior in inter-connected channel geometries. We further develop design principles that can be employed to build complex droplet networks with desired characteristics.

Firstly, the basic principles of single-phase and muti-phase flow at low-Reynolds number in confined geometries are described. Numerous hydrodynamic principles can be exploited via geometrical rearrangements that lead to a rich set of observable physical phenomena. A brief overview of low-Reynolds, laminar, reversible hydrodynamics for Newtonian fluids is covered. To enable design of complex droplet networks, the notion of hydraulic resistance of a fluid channel to single phase flow is introduced. This notation is further expanded to include more complex flow behavior arising by the presence of multi-phase flow.

Reduced order models for traversing droplets and bubbles in channel networks are then described. This allows us to consider microfluidic channel networks as non-linear resistor 
networks with droplets and bubbles traveling as point source of non-linearities. We further highlight the dependence of hydraulic resistance of multi-phase flow on viscosity ratio of dispersed and continuous phase, surfactant concentrations and Capillary number. Finally we briefly review the effect of external fields including temperature and electric fields on the flow dynamics of droplet networks.

\subsection{Low Reynolds number Stokes flow}

Cover the hydrodynamics of low Reynolds number flow of droplets and bubbles in complex channel networks. Introduce the ideas of inter-connectedness and hydrodynamic interactions in Stokes flow. Introduce various means and mechanisms to perturb flow using temperature, viscosity, surfactants.

Continuum hydrodynamics describes the laws of motion for fluid elements. Discrete quantities used to describe mechanics of solids are replaced by continuum fields such as density $\rho$, velocity vector $v$ and so on. The continuum version of Newton's law $F=m a$ is given by the Navier-Stokes equation for a unit volume

$$
\rho\left(\frac{\partial u}{\partial t}+u \cdot \nabla u\right)=-\nabla p+\eta \nabla^{2} u+f
$$

For low Reynolds number conditions when inertial forces are smaller as compared to viscous forces, the above equation can be further simplified into the Stokes equation by neglecting the inertial terms

$$
-\nabla p+\eta \nabla^{2} u+f=0
$$

Mass conservation for incompressible fluids also requires

$$
\nabla \cdot u=0
$$

Solving the above set of equations in a relevant reference frame provides a $3 \mathrm{D}$ velocity vector $u$ for all points in the fluid volume. 

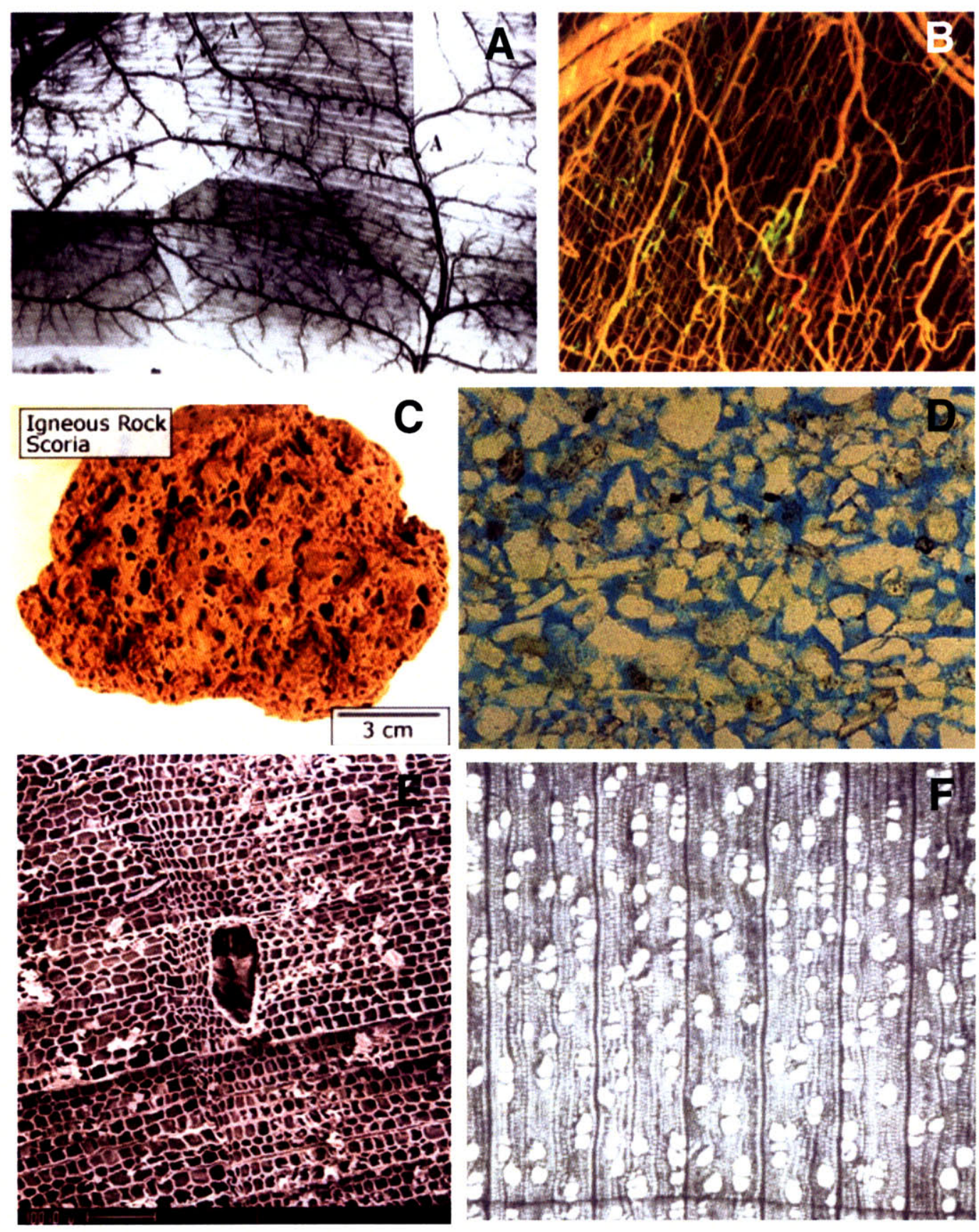

Figure 2-1: Channel network with complex topology and connectivity found in animate and in-animate natural systems. (A) Microvascular network of cat sartorius muscle (Image courtesy I.A Torres Filho). (B) Microvascular cast of hamster cremaster muscle infused with FITC-BSA (Image courtesy S. Segal) (C) Porous Ingeous rock (Scoria) (D) Porous Pilocene sandstone filled with blue epoxy during sample preparation (Image cortsey T.N. Diggs). $(\mathrm{E}, \mathrm{F})$ SEM micrographs of micro-channel structure in hard wood in vertical and horizontal cross-sections (Image courtesy H.A. Core, W.A. Cote and A.C. Day, Wood structure and identification). 


\subsection{Resistance of a droplet in a channel}

\subsubsection{Complete vs. Partial Wetting}

Contact-line region in micro-scale geometries dominate the flow dynamics. Since the length scale of contact lines are similar to flow geometries, large stresses are introduced due to fluid slip present at the triple contact point. Furthermore, the contact line resistance depends critically on microscopic defects present on the surface. Thus any chemical and mechanical variability of surface properties of microchannels results in unpredictable dynamics of multiphase flow with wetting contact lines. Contact lines in multi-phase microfluidic flows can be simply avoided by using a wetting continuous phase: a thin fluid film of continuous phase coating the microchannels.

Other than difficult to predict dynamics of contact-lines in mmulti-phase flow: several other advantages of a using completely wetting continuous phase fluids exist. Firstly, in the context of digital microfluidics, samples contained in the dispersed phase are truly isolated and never see the surface of the micro-channel. This avoids the problem of "sample dispersion", commonly faced in the world of microfluidics. Secondly valuable samples (biomolecules) are routinely lost either via sticking on the channel surface or bulk flow into the surrounding substrate (as is the case of porous substrates like PDMS). Since a true nanoliter sized container can be realized by using droplets and bubbles dispersed in a wetting continuous phase, various biochemical assays requiring strict compartmentalization of biological reagents can be easily ported to digital microfluidics. For all the above reasons, we only consider the case where one fluid completely wets the channel walls (continuous phase) and the other fluid is dispersed as drops and bubbles (unless otherwise noted).

\subsubsection{Resistance of a clean bubble: Inviscid dispersed phase}

We follow the formulation of flow of an inviscid dispersed phase (for e.g. air displacing a capillary filled with water) in a circular tube filled with continuous phase with viscosity $\mu$ by Manga et al. [13]. Bretherton [1] studied the case of displacement of continuous phase by 
an inviscid fluid at low capillary numbers, $C a<10^{-2}$. Here we consider two parameters to characterize the flow, capillary number $C a$ and volume fraction of dispersed phase $\phi$. Here we only consider the case of discrete non-wetting bubbles in the channel, thus ensuring presence of a thin continuous phase fluid film on the smooth channel walls.

Bretherton [1] evaluated the pressure drop for a bubble in a channel

$$
\Delta P_{b} \simeq 3.58 \frac{\sigma}{r}(3 C a)^{2 / 3}
$$

for $C a<10^{-2}$. Chang et al. [11] evaluated the next order term for higher $C a$

$$
\Delta P_{b} \simeq \frac{\sigma}{r}\left[3.58(3 C a)^{2 / 3}-9.07 C a^{0.95}\right]
$$

for $C a<10^{-1}$.

Furthermore, the height of the thin film left behind by the continuous phase between the bubble and the wall is given by

$$
h_{0}=0.643 r(3 C a)^{2 / 3}
$$

not including the effect of non-hydrodynamic forces such as van der Waals forces that come into effect for thin films smaller than $10-100 \mathrm{~nm}$.

At low Reynolds number stokes flow, the hydraulic resistance of continuous phase for a tube segment of length $L$ is given by

$$
Q=\frac{\pi \rho r^{4}}{8 \mu L} \Delta P
$$

with $\Delta P$ is the driving pressure gradient. If a bubble is present in the tube, the formulation for conductivity of the tube is modified as

$$
Q=\frac{\pi \rho r^{4}}{8 \mu\left(L-l_{b}\right)}\left[\Delta P-\sigma \Delta P_{b}(Q)\right]
$$

where $l_{b}$ is the length of the bubble. Furthermore, for a clean bubble interface the velocity 
of the bubble $u$ to the surrounding wetting fluid $v$ can be expressed as

$$
v=(1-w) u
$$

where $w \simeq 1.29(3 C a)^{2 / 3}$ and $v=Q / \rho \pi r^{2}$. Thus we can write the conductivity of the channel with a bubble as

$$
Q=\frac{\pi \rho r^{4}}{8 \mu\left(L-l_{b}\right)}\left[1-\sigma \frac{\Delta P_{b}(Q)}{\Delta P}\right] \Delta P
$$

where conductivity is a function of the pressure gradient and the flow rate and hence nonlinear in nature (when compared to the linear term for conductance of single phase flow in a circular tube). At large $C a$ bubble speed is drastically slower than the velocity of the continuous phase. For smaller $C a$ pressure drop due to a bubble forms a significant percent of the total pressure drop. This can be understood since the pressure drop across a bubble is proportional to $C a^{2 / 3}$, and thus proportional to $u^{2 / 3}$. The pressure drop across a tube with no bubble is proportional to $v$. Thus as $C a$ decreases, pressure drop across a bubble increases faster than pressure drop in a tube without a bubble [13].

\subsubsection{Effect of viscosity}

Hodges et al. [6] considers the effect of viscosity of a fluid droplet (viscosity $\lambda \mu$ ) in a circular tube radius $R$ surrounded by a wetting continuous fluid (viscosity $\mu$ ). Here we present the scaling laws for the same highlighting the effect of viscosity. A thin film of continuous fluid around the droplet is given by $\epsilon R$. Hodges et al. show that the scaling $\epsilon \sim C a^{2 / 3}$ still holds, for all values of $\lambda$ with a unity coefficient, found using lubrication theory. As $\lambda$ increases, film thickness increases by a factor $4^{2 / 3}$ to plateau for $C a^{-1 / 3}<<\lambda<<C a^{-2 / 3}$. The factor falls to $2^{2 / 3}$ as $\lambda \rightarrow \infty$.

For a viscosity ratio $\lambda$, effective resistance of a channel with discrete liquid slugs (number of slugs $n$ ) can be approximated as [7]

$$
R_{e f f}=12.8 \frac{l}{b^{3} w} \mu(\lambda \phi+1-\phi)+n_{d} 3.15 g(\lambda) \frac{\gamma}{b} \frac{C a^{2 / 3}}{q}
$$




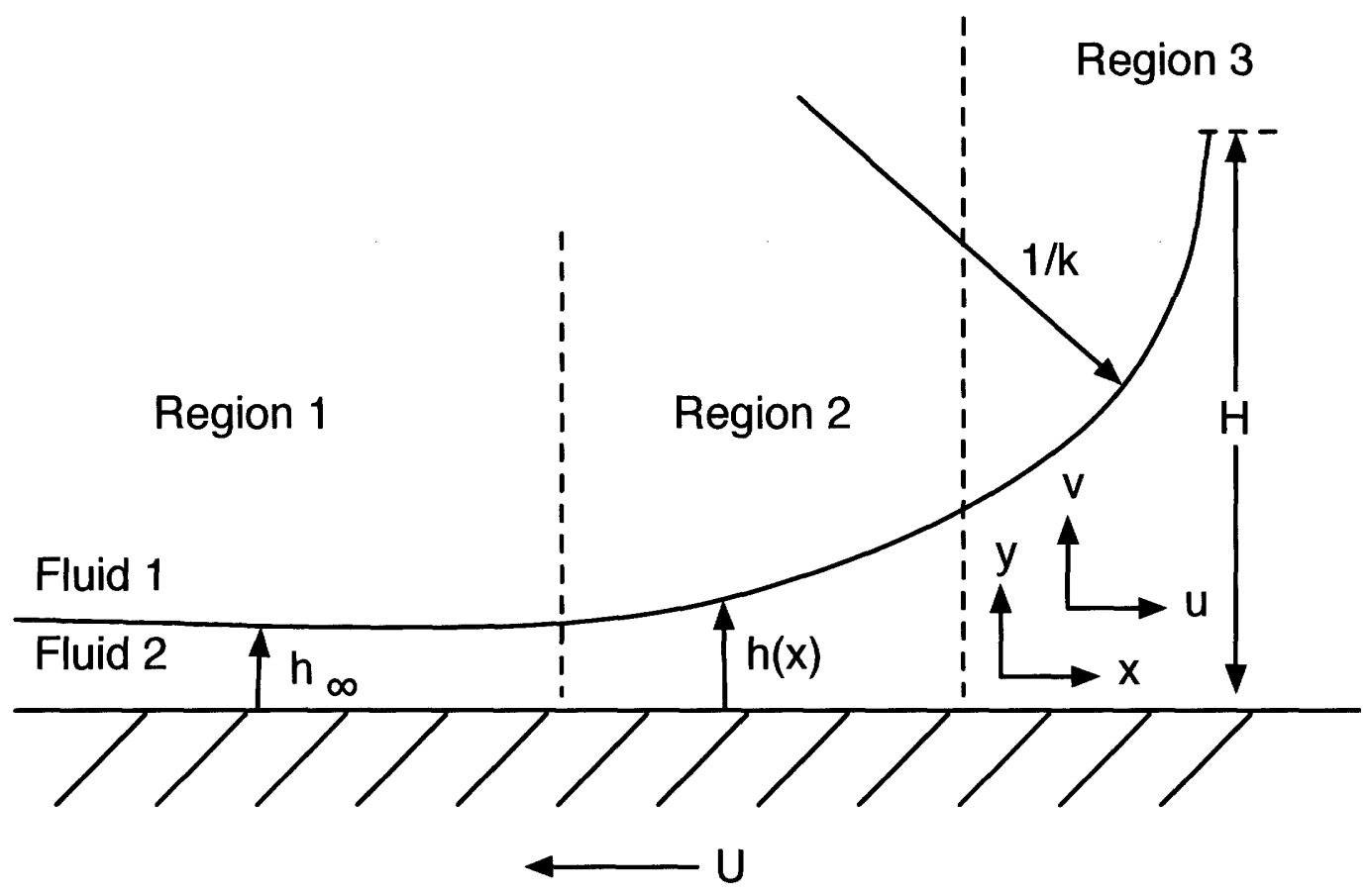

Figure 2-2: Fluid filled in a tube displaced by another non-wetting fluid. Three regions depict various dominant forces governing the interface shape for the dispersed fluid.

for a square channel with width $w$ and height $b, \gamma$ is surface tension, $\phi$ is volume fraction, $\mu$ is viscosity of continuous phase, $C a$ is capillary number, $\lambda$ is viscosity ratio of dispersed to continuous phase $(0<\lambda<\infty) . g(\lambda)$ is a function of $\lambda$ and varies between 1 to $1.5[6]$. Thus $g(\lambda)$ will only have a small effect as viscosity of the dispersed phase is changed.

For small viscosity ratio $\lambda<<1$ and small volume fraction $\phi<<1$, flow resistance to a droplet can be approximated as [7]

$$
R_{D} \sim 0.25\left(\frac{b f}{u}\right) C a^{-1 / 3}
$$

providing an approximate expression for increases in hydraulic resistance considering the increased viscosity of the dispersed phase. 


\subsubsection{Droplets at a $\mathrm{T}$ junction}

Let us first consider the simplest channel geometry with one inlet port and two symmetric outlet ports, i.e. a $\mathrm{T}$ junction. For non-breaking droplets, this was initially studied in mill-fluidic channels $(600 \mu \mathrm{m})$ by Ajdari et al. [2]. For single phase flow of Newtonian fluids at low Reynolds numbers $\left(R e 10^{-1}\right)$, this is a simple setup with flow from the inlet separating into two outlet streams - proportionately to the flow resistance of the outlet channels (assumed equal in this case). We now look at a more complex situation, where we allow droplets or bubbles to enter the inlet port as a train and observe the outlets as they leave the two exit ports. We only consider the small capillary number limit $\left(\mathrm{Ca} 10^{-2}\right)$, where the viscous stresses are small as compared to the surface tension force which keep the droplets from breaking up at the junction due to viscous force induced stretching. Thus droplets arriving at a junction make a binary decision of going either left or right. This is a distinctly non-linear event since one of the two outlets is chosen by individual droplets.

A simple qualitative picture for such a device can be produced by considering a hydrodynamic model for the $\mathrm{T}$ junction that includes resistance to flow due to presence or absence of a droplet in a channel. A droplet that chooses the path of least resistance amongst the two outlets, in term increases the resistance of that exit channel. If instantaneous resistance of this channel is higher due to presence of this droplet, majority of the net flow rate at the junction can switch to a outlet with higher resistance. Such a simple feedback mechanism leads to a non-linear behavior of droplets arriving at a $\mathrm{T}$ junction. The two critical factors that influence the behavior include net increase in hydraulic resistance due to presence of a bubble or a droplet in a fluidic channel and frequency (rate of arrival) governing packing density of a train of droplets in a channel. At low dilution frequency. the junction acts like a filter directing all the droplets to the exit channel with smaller resistance - while at higher frequency the distribution is more complex and dynamic (depending on the exact sequence of droplets) [2]. 


\subsubsection{Numerical formulation}

Given a complex channel network topology, is it possible to predict the dynamics of droplets and bubbles at low Reynolds numbers? Two components that play a significant role in such a system are; increased resistance to flow due to individual droplets or bubbles in a channel and hydrodynamic interaction of these droplets in complex geometries, for e.g. a simple loop structure.

Let us first consider a pressure drop due to a bubble traveling in a channel. This provides a formulation for total hydrodynamic resistance of a bubble in a channel. Consider the net pressure drop as $\Delta P_{\text {total }}$

$$
\Delta P_{\text {total }}=\Delta P_{n b}+\Delta P_{b o d y}+\Delta P_{c a p s}
$$

where $\Delta P_{n b}$ represents the pressure drop for the regions containing no bubbles, $\Delta P_{b o d y}$ represents the pressure drop due to the region containing the body of the bubble and $\Delta P_{\text {caps }}$ accounts for the curved caps that cover the front and the back of a bubble. Different

hydrodynamic forces dominate in the three given regions, providing a different scaling laws for the total pressure drop in channel given by $\Delta P_{\text {total }}$. Considering the bubble size to be larger than the channel width (thus in Bretherton regime), the pressure drop can be written as $[4]$

$$
\begin{aligned}
\Delta P_{n b} & =a \mu V_{n b}\left(L_{\text {total }}-L_{\text {cap-to-cap }}\right) / H^{2} \\
\Delta P_{\text {body }} & =b \mu V_{n b} L_{b o d y} / H^{2} \\
\Delta P_{c a p} & =c \mu V_{n b} C a^{-1 / 3} / H
\end{aligned}
$$

where $H$ is the channel height, $\mu$ is the viscosity of the continuous phase, $C a$ is the capillary number.

For a given topology network, a set of equations can be solved with the following conserva- 


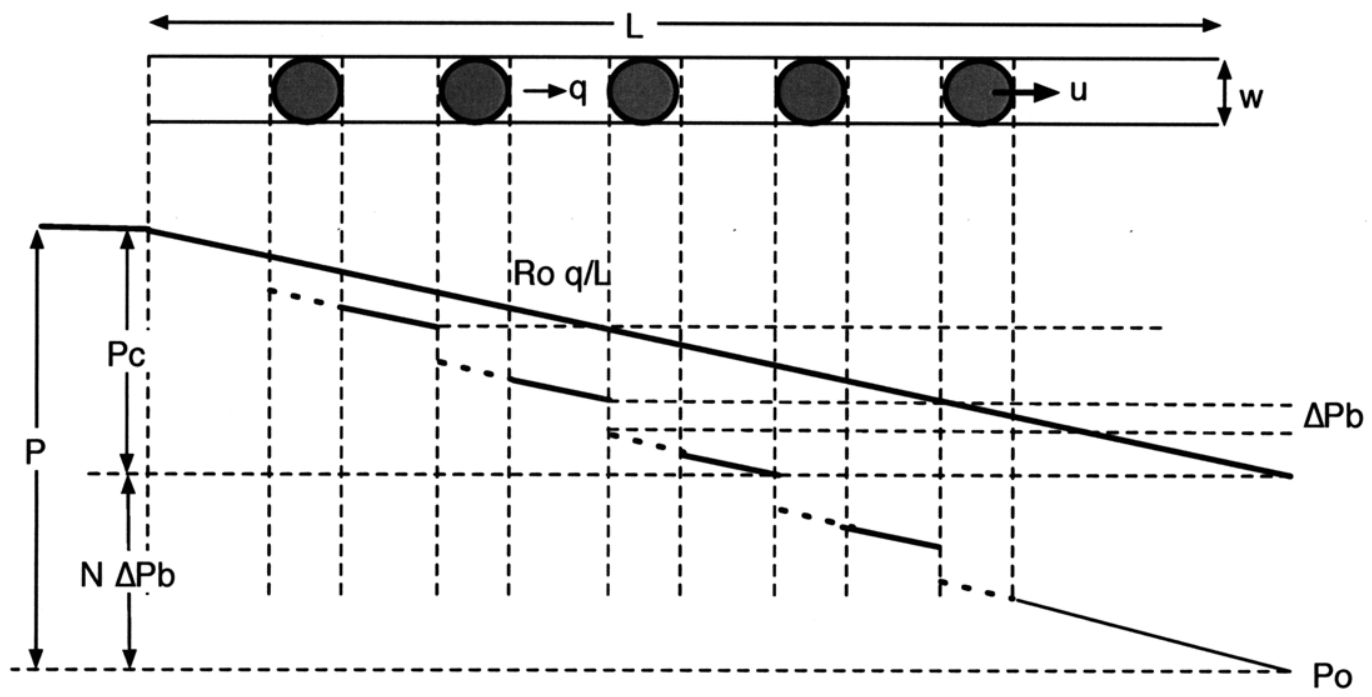

Figure 2-3: Pressure drop due to a train of droplets in a channel. Adapted from Sullivan et al. [14]

tion rules on nodes and channels. At every node

$$
\Sigma \epsilon_{i} Q_{i}=Q_{e x t}
$$

which ensures conservation of flow, and at every channel

$$
\Delta P=P_{\text {oin }}-P_{\text {oout }}=R_{i} Q_{i}
$$

with hydrualic resistance of a channel with a drop or a bubble modified as

$$
\begin{aligned}
R_{i} & =\bar{R}_{i}+n_{i} R_{d} \\
V_{i} & =\beta Q_{i} / S
\end{aligned}
$$

where $\mathrm{S}$ is the cross-section of the channel, $\beta$ is a proportionality constant, $n_{i}$ represents the number of droplets in a channel, $R_{d}$ depicts the resistance of a droplet in a channel. 


\subsection{Influence of other parameters}

\subsubsection{Effects due to surfactants}

Presence of surfactant molecules on the fluid interface, greatly increases the hydraulic resistance of a bubble or a droplet in a fluidic channel. Coupling of flow due to bubble motion results in a non-uniform distribution of surfactant molecules on the interface. Thus a gradient in surfactant concentration, and hence surface tension modifies the dynamics and hydraulic resistance of the traversing bubble. Most importantly, presence of surfactants on the interface change the boundary condition, coupling flow of surrounding fluid to surfactant concentration.

Here we follow the outline by Herbolzheimer [3], to determine the resulting pressure drop across a bubble, in presence of a surfactant. The geometry we consider here is a long closed bubble with front and back interface, with continuous fluid phase completely wetting the channel walls (distance $2 a$ ). The bubble forms a thin lubrication film along the bubble, of length $l$. In the above geometry, surfactant molecules on the interface are swept backwards to the rear end of the interface. Thus the surface tension at the rear end is significantly lower when compared to the front end. This surface tension gradient across the length of the bubble exerts an attractive force, increasing the pressure drop across the bubble.

Let us reconsider the results for a clean interface, i.e. surfactant free bubble in a channel - as worked by Bretherton [1]. A clean interface at the surface of the bubble (with the air viscosity being so low) can not sustain any shear stress. Thus majority of viscous dissipation occurs at the front and the rear end of the bubble. Bretherton evaluated the true mean curvature of front meniscus (at small capillary number) to be $2 / a\left[1+1.79(3 C a)^{2 / 3}\right]$, leading to a dynamic pressure drop of $3.58(\sigma / a)(3 C a)^{2 / 3}$. Similarly for the rear end of the bubble, the dynamic pressure is given by $-0.94(\sigma / a)(3 C a)^{2 / 3}$. Thus the total pressure drop can be evaluated to be [10]

$$
\Delta p=9.40 C a^{2 / 3} \frac{\sigma}{a}
$$

The above formulation provides an estimate of pressure drop due to a clean bubble - which 


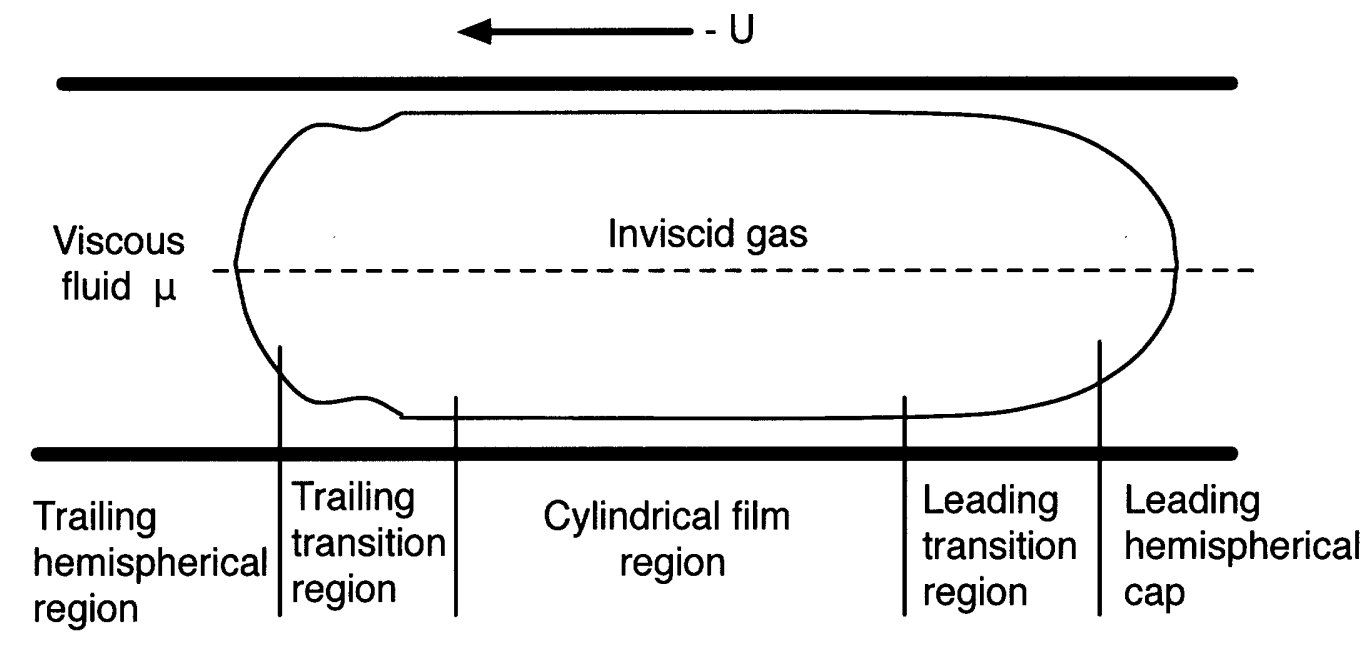

Figure 2-4: Interface shape due to an inviscid bubble traveling in a channel, with six distinct regions marked by the trailing and the leading edge of the bubble.

is independent of the length of the bubble and goes as $\sigma^{1 / 3}$, thus reducing as surface tension decreases (which is the case when surfactants are added).

In the case of surfactants present on the interface, the surfactant film behaves like an incompressible thin solid, with tangential velocity on the interface being zero (no-slip boundary condition). This is in contrast to the zero shear stress condition for a clean bubble. Thus the viscous dissipation in the thin lubrication film dominates the pressure drop across the bubble, leading to higher hydraulic resistance of surfactant laden bubbles. Balancing the pressure difference with the viscous drag in the thin lubrication film, we can write

$$
\pi a^{2}(\Delta p)=2 \pi a l \mu U / \delta_{f}
$$

By experimental observations, addition of surfactants increases the pressure drop across a bubble traveling in a channel.

\subsubsection{Instabilities due to low surface tension}

Addition of surfactants, commonly used in droplet microfluidic systems to avoid coalescence and provide a bio-compatible interface for dispersed phase, results in reduction of 


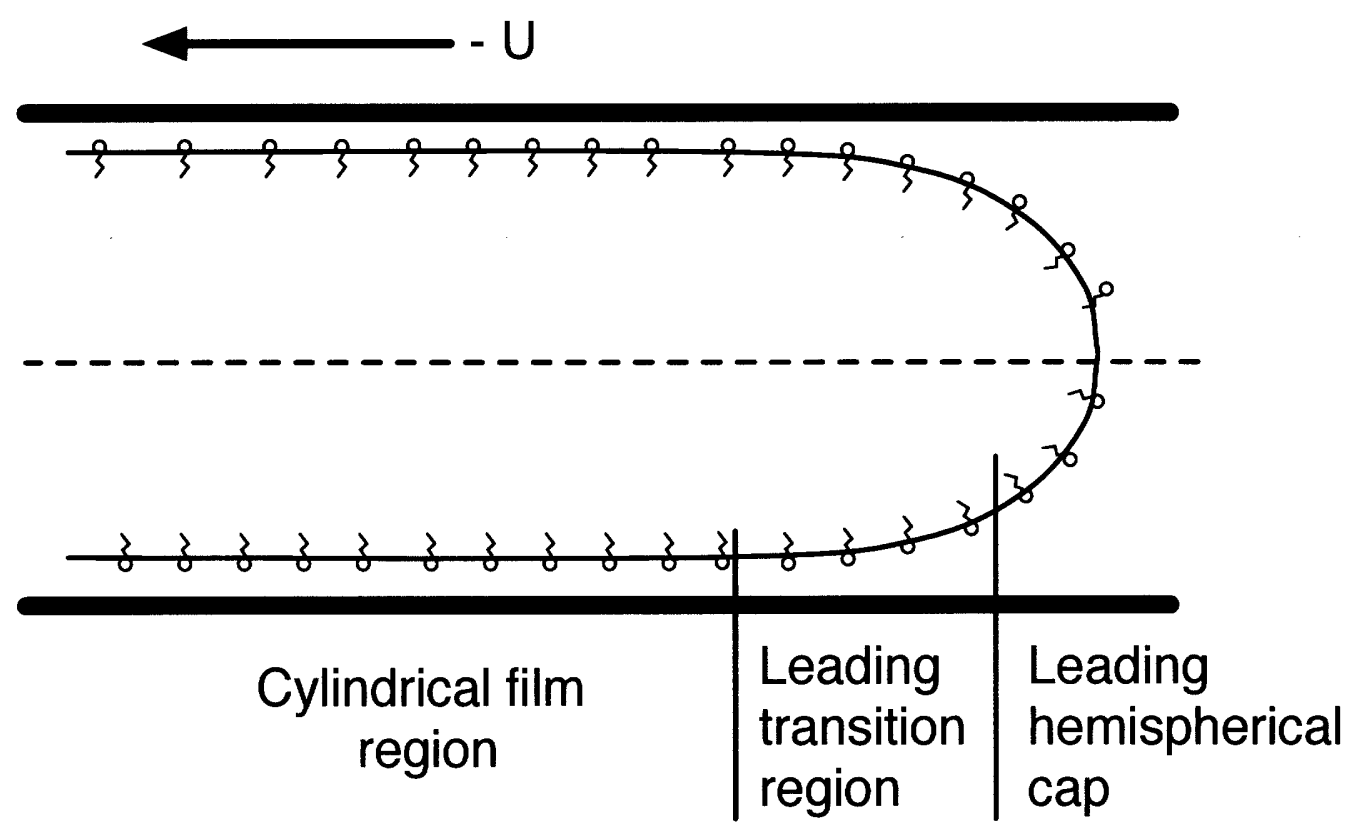

Figure 2-5: Surfactant distribution on the interface of a inviscid bubble in a wetting fluid.

surface tension at the interface. Since many bio-molecules also act as surfactants, the net surface tension can be further reduced due to presence of surfactants in both dispersed and continuous phase. Low surface tension between dispersed and continuous phase can lead to instabilities, resulting in commonly observed patterns formed in droplet trains. From a perspective of droplet microfluidics, this can be a limitation. Thus understanding the effects and means of suppressing these instabilities is crucial.

For very low surface tension $(0.01 \mathrm{mN} / \mathrm{m})$ - tip streaming from droplets can be observed in microfabricated channel networks [5]. Two distinct types of instabilities that have been observed - Rayleigh-Plateau instabilities for elongated droplets and shear driven instabilities due to excessive shear stress at the interface due to top and bottom walls of the channels [5]. Narrow confinement in microfluidic channels has been previously reported to suppress Rayleigh-Plateau instabilities [12,9], when compared to stretched droplets in unbounded fluid. Extensional flow above a threshold, in microfluidic T junctions [8] or Hele-Shaw cell type geometries [5], leading to elongation of dispersed phase commonly results in drop breakup.

Consider a droplet dispersed in a continuous phase, sheared due to a extensional flow field. 
In the static case, when shear is exerted on the droplet - the interface deforms into a spherical shell minimizing the interfacial surface energy of the system. For shear induced deformation of a droplet, the surface tension opposes the deformation until a point at which the interface is destabalized and surface tension can no longer balance the shear stress, resulting into onset of instabilities and potential breakup of the droplet. For a droplet subjected to specific shear rate, Capillary number $C a$ can be written as $C a=\mu \dot{\eta} R / \gamma$ where $\dot{\eta}$ is shear rate and $R$ is radius of the droplet. Thus the largest stable droplet for a critical Capillary number $C a_{c r}$ can be written as $R^{*}=\gamma C a_{c r} / \mu \dot{\eta}$. The above formulation does not consider any confinement effect and is only valid for geometries where shear is induced by bulk flow and dispersed phase is not pinched by confinement geometries. Droplet microfluidics typically operate in flow regime where droplet radius in unbounded state $2 R>w$ where $\mathrm{w}$ is the width of the channel. Thus confinement effects need to be accounted for while considering the stability of dispersed phase.

A common but slightly paradoxical observation of elongated droplets traveling in microchannels, is the stability of these droplets - even though the length to width ratio is larger than the traditional $l / w$ ratio of $\pi$, where 1 is the length of the drop and $w$ is the width of the drop [8]. With increasing confinement, wavelength of the most unstable perturbations for Rayleigh-Plateau instability increases while the speed of the collapse decreases. The instability of a droplet traveling in a confined channel can be further characterized by the characteristic Capillary number $C a$, given by $\mu v / \gamma$, where $\mu$ is the viscosity, $\mathrm{v}$ is the velocity of the continuous fluid and $\gamma$ is the surface tension of the interface. At higher Capillary number, the instabilities observed in traversing droplets are further influenced by redistribution of surfactant molecules on the free interface - resulting in a surface tension gradient along the interface. For a higher Capillary number, surfactants accumulate at the trailing edge of droplets - leading to further reduction of surface tension locally. The interface is thus easily destablized by shear forces generated due to confined channel geometries. Droplets traversing with the flow, even though longer than the Rayliegh-Plateu limit (with aspect ratio larger than $\pi$ ) do not break in straight channels. The stability of there drops can be attributed to flow around the drop [8]. For a flow in the continuous phase, disturbances on the interface can be convected to the end of the drop before they grow to be large enough 
to cause droplet breakup. Furthermore, nearby walls suppress any deformations on the interface that could lead to growing instability.

\subsubsection{Gravity}

Influence of gravity on flow parameters can be quantified easily by evaluating the operating Bond number, $B o=\delta \rho g R^{2} / \gamma$, where $\delta \rho$ is difference between component density, $g$ is gravitational acceleration and $R$ is radius of the drop. For most droplet microfluidic systems, $B o$ number varies from $10^{-3}$ to $10^{-4}$. Since $B o<<1$, gravitational effects are essentially

insignificant . For the very same reason, orientation of the microfluidic device does not effect the flow dynamics of droplets and bubbles in microfluidic networks. 


\section{Bibliography}

[1] F. P. Bretherton. The motion of long bubbles in tubes. Journal of Fluid Mechanics, 10:166-188, 1961.

[2] Galder Cristobal, Jean-Philippe Benoit, Mathieu Joanicot, and Armand Ajdari. Microfluidic bypass for efficient passive regulation of droplet traffic at a junction. Applied Physics Letters, 89:034104, 2006.

[3] Herbolzheimer E. The effect of surfactant on the motion of a bubble in a capillary. AICHE Annual meeting, New York, 1987.

[4] Michael J. Fuerstman, Ann Lai, Meghan E. Thurlow, Sergey S. Shevkoplyas, Howard A. Stone, and George M. Whitesides. The pressure drop along rectangular microchannels containing bubbles. Lab on Chip, 7:1479-1489, 2008.

[5] Michinao Hashimoto, Piotr Garstecki, Howard A. Stone, and George M. Whitesides. Interfacial instabilities in a microfluidic hele-shaw cell. Soft Matter, 4:1403-1413, 2008.

[6] S.R. Hodegs, O.E. Jensen, and J.M. Rallison. The motion of a viscous drop through a cylindrical tube. J. Fluid Mech., 501:279-301, 2004.

[7] F Jousse, R Farr, D Link, M.J. Fuerstman, and P. Garstecki. Bifurcation of droplet flows within capillaries. Physical Review E, 74:036311, 2006.

[8] D. R. Link, S. L. Anna, D. A. Weitz, and H. A. Stone. Geometrically mediated breakup of drops in microfluidic devices. Phys. Rev. Lett., 92(5):054503, Feb 2004. 
[9] J.A. Pathak and K.B. Migler. Droplet-string deformation and stability during microconfined shear flow. Langmuir, 19(21):8667-8674, 2003.

[10] Ronald F. Probstein. Physicochemical Hydrodynamics: An Introduction. WileyInterscience, 1994.

[11] J. Ratulowski and H.C. Chang. Transport of gas bubbles in capillaries. Physics of fluids, 1:1642-1655, 1989.

[12] Y. Son, N.S. Martys, J.G. Hagedorn, and K.B. Migler. Suppression of capillary instability of a polymeric thread via parallel plate confinement. Macromolecules, 36(15):5825$5833,2003$.

[13] J. Stark and M. Manga. The motion of long bubbles in a network of tubes. Transport in Porous Media, 40:201-218, 2000.

[14] M.T. Sullivan and H.A. Stone. The role of feedback in microfluidic flow-focusing devices. Phil. Trans. R. Soc. A, 366:2131-2143, 2008. 


\section{Chapter 3}

\section{Microfluidic bubble logic}

Based on a published article "Microfluidic Bubble Logic", Prakash M, Gershenfeld N, Science Vol. 315, pp. 832-835, Feb. 2007.

\section{$3.1 \quad$ Abstract}

We demonstrate universal computation and bistability in an all-fluidic two-phase microfluidic system. Nonlinearity is introduced in an otherwise linear, reversible, low Reynolds number flow via bubble-to-bubble hydrodynamic interactions. A bubble traveling in a channel represents a bit, providing us with the capability to simultaneously transport materials and perform logical control operations. We demonstrate bubble logic AND/OR/NOT gates, a toggle flip-flop, a ripple counter, a timing restoration device, a ring oscillator and an elctro-bubble modulator. These show the nonlinearity, gain, bistability, synchronization, cascadability, feedback and programmability required for scalable universal computation. With increasing complexity in large scale microfluidic processors, bubble logic provides an on-chip process control mechanism integrating chemistry and computation. 


\subsection{Introduction}

Microfluidic "lab-on-a-chip" devices, where picoliters of fluids can be precisely manipulated in microscopic channels under controlled reaction conditions, have revolutionized analytical chemistry and biosciences. Recent advances in elastomeric pneumatic micro-valves [24] and large scale integration [22] have enabled complex process control for a wide variety [17, 2] of applications in single phase micro-reactors. Pneumatic elastomeric micro-valves require external macroscopic solenoids for their operation. Cascadability and feedback (where a signal acts on itself), which are common in electronic control circuits, is currently lacking in microfluidic control architectures.

Another problem in microfluidics is reagent interaction with channel walls which causes dispersion and non-uniform residence time distribution due to Poiseuille flow (parabolic flow profile). Several reaction chemistries have been implemented in segmented-flow twophase micro-reactors, where individual nanoliter droplets traveling inside microchannels are used as reaction containers[14, 28]. Di-electrophoretic [10] and electrostatic[19] force based external control schemes have been proposed on-chip droplet management but they all require independent control of a large number of external electrodes and provide only single gate level control which limits scalability. Due to the lack of a scalable control mechanism, segmented-flow micro-reactors are still designed as simple linear channels with very limited spatial and temporal control when compared to a single-phase micro-reactor employing pneumatic micro-valves [22]. Flow control that exploits the dynamics of droplets inside mi-

crochannels would make high-throughput screening and combinatorial studies possible[15], but preliminary implementation of passive control techniques $[21,7]$ have not provided single droplet control.

We demonstrate bubble logic through an implementation of universal Boolean logic in physical fluid dynamics. This provides us a droplet-level, internal, inherently digital, flow control mechanism for microfluidic processors. Our approach does not require off-chip components and can scale to complex structures. A bubble traveling in a micro-channel can represent a bit of information as well as carry a chemical payload, thus making it possible to integrate 
chemistry with computation for process control. Using such bubbles we demonstrate the nonlinearity, gain, bistability, synchronization, cascadability and feedback required for a scalable universal logic family. Bubble logic preserves the information representation from input to output, thus devices can be cascaded allowing implementation of combinatorial and sequential Boolean circuits. A bubble can be transported to a desired location in a complex microfluidic network via a series of cascaded logic gates corresponding to an equivalent Boolean circuit. Such an implementation of digital logic for process control also provides us with a modular design approach for droplet control architectures, where individual logic gates can be connected in a hierarchal structure.

Several families of logic circuits utilizing nonlinearities in physical fluid dynamics have been reported in the past. Fluidic logic from the 1960's [6,3] was dependent on the inertial properties of fluid flow thus requiring high Reynolds number flow. Such fluidic gates were used to build a trajectory controller, an all-fluidic display, non-destructive memory and a simple computer [3]. With miniaturization, which was necessary for higher operating speeds and integration, it was impossible to maintain high Reynolds number flow in microscopic geometries. Fluids with polymer additives have been used to implement a constant flow source and a bistable gate $[11,12]$ but the operation of these devices is dependent on nonNewtonian fluid properties. Change in flow resistance has been used [25] to build Boolean logic in a single-phase Newtonian fluid, but since its input and output representation are not the same these devices could not be cascaded. Bubble logic, based on hydrodynamic bubble-to-bubble interactions, is similar in bit representation to theoretical billiard ball logic[8] based on the elastic collision of particles, and magnetic bubble memory[5] relying on interactions of magnetic domains in garnet films. These schemes all conserve information because during a logic operation a bit is neither created nor destroyed.

\subsection{Microfluidic channel networks}

Microscopic bubbles traveling in complex fluidic networks exhibit rich nonlinear dynamics. Pressure-driven flow behavior of bubbles in an interconnected microfluidic network can be 
described using a simplified dynamic flow resistance model[16]. Single phase flow resistance of a channel at low Reynolds number can be approximated as $\Delta p / Q \propto \mu L / h^{3} w$ where $\Delta p / L Q$ is defined as the hydraulic resistance per unit length, $\mu$ is dynamic viscosity, and $h$ and $w$ are the height and width of the micro-channel. The presence of a bubble in a channel drastically increases this flow resistance. The pressure drop due to a long bubble flowing in a channel, where the bubble radius in an unbounded fluid is greater than the channel width and the continuous phase completely wets the channel surface, is nonlinear and is proportional to $\Delta p \propto \sigma / w\left(3 C a^{2 / 3}\right)[4,27]$, where $C a$ is the Capillary number $(C a=\mu u / \sigma)$, $\mu$ is the dynamic viscosity, $u$ is the flow velocity of the continuos phase, $w$ is the channel width and $\sigma$ is the surface tension between liquid and gas phase. For small flow rates this increased flow resistance is primarily due to viscous dissipation in the thin film of liquid surrounding the bubble. With the presence of surfactant molecules on the air-water interface, viscous dissipation in the lubrication film further increases due to the no-slip boundary conditions at the interface. In this case, the pressure drop across a finite length bubble is also linearly dependent on the bubble length until it reaches a critical value, beyond which it is constant[20]. When a bubble traveling in a micro-channel arrives at a bifurcation with low Capillary number (where the bubble does not split due to surface tension dominating the viscous stress), it chooses the branch with highest instantaneous flow $[18]$.

\subsection{Universal Logic Gates}

With an increased flow resistance due to the presence of a bubble in a micro-channel, flow lines in surrounding interconnected channels can be perturbed. These perturbations can hence be used to route another bubble stream resulting in hydrodynamic interactions between bubbles. Navier-Stokes equations describing low Reynolds number flow are linear due to negligible inertial terms. Nonlinearity in such a system arises from the introduction of interfacial force terms from the boundary conditions due to the presence of a free surface at the fluid interfaces[23]. Such nonlinear time-dependent interactions are the basis of our bubble logic gates. We exploit such interactions to build AND, OR and NOT gates, forming 
a universal Boolean logic set. Since bubbles are neither produced nor destroyed during a bubble logic operation, the number of bubbles is conserved from input to output for a given device. In the implementation described here we use water as the liquid media (with added surfactant $2 \%$ by w/w Tween 20 ) and nitrogen bubbles. We fabricated planar bubble logic devices in PDMS (poly-dimethyl siloxane) using single-layer soft-lithography and plasma bonding to Pyrex substrates. Fig. 3-1 (top row) shows an AND/OR bubble logic gate which evaluates both AND $(\cdot)$ and OR (+) simultaneously (necessary to satisfy bit conservation). A single bubble arriving from either $A$ or $B$ at the junction will choose the wider channel, corresponding to $A+B$. When bubbles arrive from both $A$ and $B$ simultaneously, both output channels contain a bubble (Fig. 3-1 top row, middle column) evaluating both $A+B$ and $A \cdot B$. The bubble arriving earlier at the junction always enters $A+B$ (the wider channel, with less resistance) increasing the output flow resistance of $A+B$, thus directing the bubble arriving later to $A \cdot B$. As shown in the time trace for all four channels of the device (Fig. 3-1 top row, last column), the two bubbles interact only if they arrive within a window $\tau_{0}$ (for this gate $\tau_{0} \sim 0.5 \mathrm{~ms}$ at $Q=0.25 \mu \mathrm{l} / \mathrm{sec}$ ) determined by the residence time of the bubble in the gate geometry. No bubble coalescence was observed in the channels, because of the stabilization of the interface by surfactant molecules.

In any logic family fan-out is necessary for the output signal from one gate to act as an input signal to multiple gates. In the case of bubble logic, this can be simply implemented by splitting bubbles at a $\mathrm{T}$ junction[18] into equal parts. Thus gain is necessary to restore signal levels (where the signal is represented by the bubble size) in a logic family. We define gain as the ratio of the volume of the output bubble to the volume of control bubble. Fig. 3-1 (bottom row) demonstrates a switching gate, which implements a NOT and an AND gate simultaneously (making it a universal gate), with gain where a small control bubble can switch the output flow direction for a larger bubble. A NOT gate is implemented as an $\bar{A} \cdot B$ gate where the value of $B$ can be set to 1 by applying a constant frequency bubble train. The switching gate consists of two counteracting asymmetries, an input channel with an asymmetric $\mathrm{T}$ junction (bottom) and a narrow stream of injected flow from the control channel (top) into the wider of the two bifurcations. By introducing a bubble in the control channel, injected side flow can be dynamically turned on and off, and hence control the 

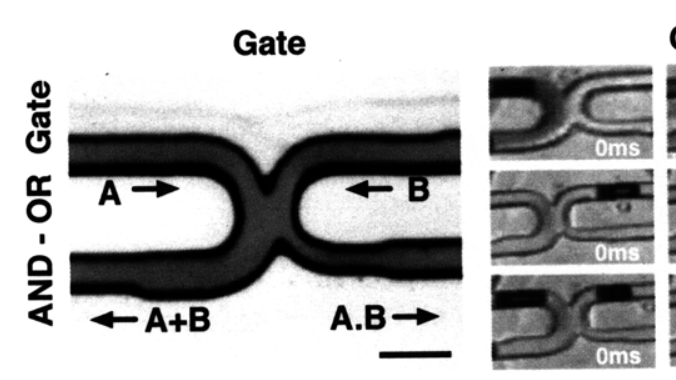

Operation
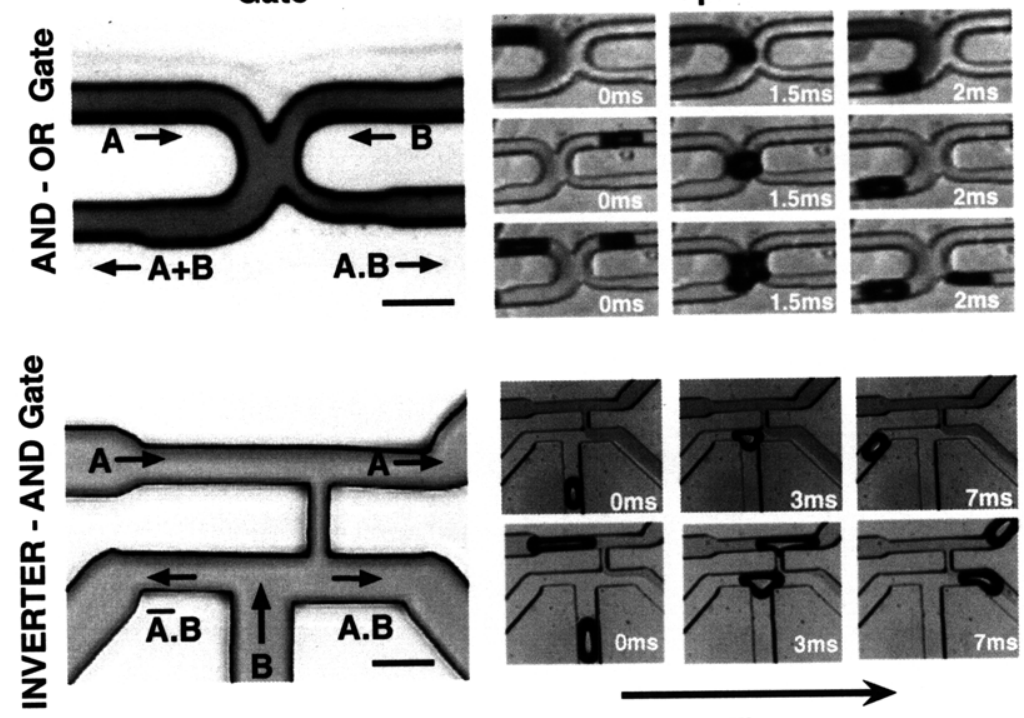

Time
Input Vs. Output
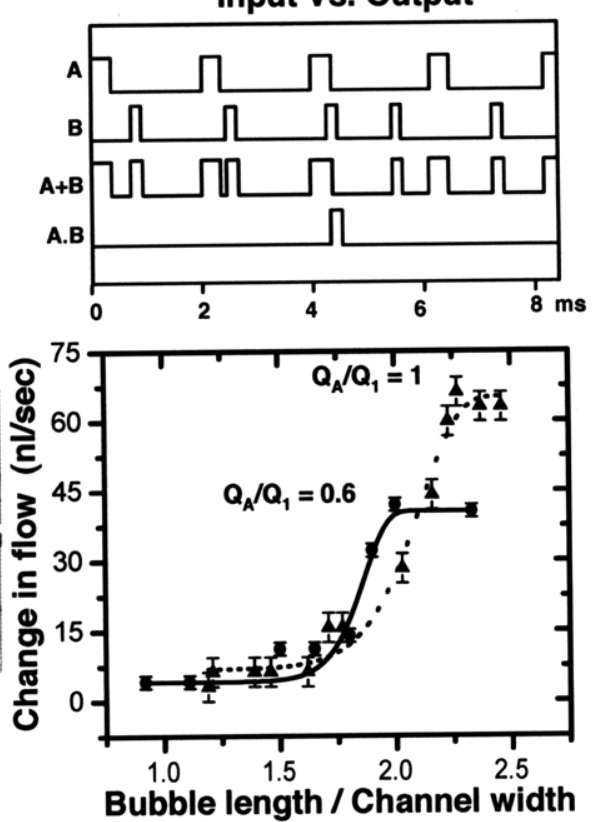

Figure 3-1: Universal microfluidic bubble logic. A bubble in a channel represents a bit. A and $\mathrm{B}$ mark the $\mathrm{T}$ junction bubble input ports to the devices (planar 2D geometry, channel height $70 \mu \mathrm{m}$ ) which are driven by a constant water input flow (with $2 \% \mathrm{w} / \mathrm{w}$ Tween 20 surfactant) via syringe pumps (Kent Scientific) and constant pressure driven nitrogen $\left(P_{0}=\right.$ $0.5 \mathrm{psi}$ ). Top row depicts a two-input AND-OR gate with symmetric input channels marked $\mathrm{A}$ and $\mathrm{B}$ (both $50 \mu \mathrm{m}$ wide) and asymmetric output channels ( marked $A+B, 65 \mu \mathrm{m}$ wide and $A \cdot B, 40 \mu \mathrm{m}$ wide) computing both AND and OR simultaneously. Scale bar $100 \mu \mathrm{m}$. Gate propagation time delay is $2 \mathrm{~ms}$ at an input flow rate of $Q_{A}=Q_{B}=0.25 \mu \mathrm{l} / \mathrm{sec}$. A signal time trace for the device is shown for a period of $8 \mathrm{~ms}$. Second row depicts a NOT gate and an AND gate implemented in the same geometry (as a universal switch gate) evaluating $\bar{A} \cdot B$ and $A \cdot B$. The device consists of two counteracting asymmetries, a T junction (100 $\mu \mathrm{m}$ wide channel input, $50 \mu \mathrm{m}$ and $65 \mu \mathrm{m}$ wide channel outputs) and an offset inlet channel ( $25 \mu \mathrm{m}$ wide) which provides variable flow from the top control channel ( $50 \mu \mathrm{m}$ wide). Scale bar $100 \mu \mathrm{m}$. A bubble in the control channel (bubble size $r_{c}$ ) significantly reduces the inlet flow switching the direction of the bubble at the $\mathrm{T}$ junction (bubble size $r_{t}$ ). Gain is defined as $r_{t} / r_{c}=1.2\left(Q_{A}=Q_{B}=0.92 \mathrm{\mu l} / \mathrm{sec}\right.$. Propagation delay time is $7 \mathrm{~ms}$. Maximum change in inlet flow as a bubble passes through the control channel is shown against dimensionless bubble size (Bubble length/Channel width) depicting a sharp nonlinearity and saturation with increasing bubble size. 
direction of flow of the output bubble arriving at the bifurcation. The change in injected flow from the control channel $(\Delta Q)$ when a bubble passes through the control channel is non-linearly related to the size of the bubble, as plotted in Fig. 3-1 (bottom row, right column) against the dimensionless bubble size (bubble length / channel width). We use this nonlinearity as a gain mechanism, allowing a smaller bubble from $A$ to switch a larger bubble coming from $B$ (Fig. 3-1 bottom row, middle column).

\subsection{Bistability}

Segmented-flow reactors often operate at kilohertz frequencies, where the limiting factor for high-thoughput screening is the rate of information extraction from individual droplets due to slower measurement processes. We present a bistable mechanism (Fig. 3-2) capable of on-demand trapping and release of individual bubbles, implemented as a toggle flip-flop. This implementation demonstrates the basic unit of memory in bubble-logic. A bubble minimizes its surface energy by adopting a shape with the smallest surface area. The toggle flip-flop geometry (Fig. 3-2 A) presents an incoming bubble with two elliptical lobes where the surface energy of the bubble is at its minimum, as shown in the plot of energy vs. time (Fig. 3-2 G). This geometry acts like a fluid dynamic bistable trap for a single bubble traveling at low Capillary and Reynolds numbers. The energy landscape of a single bubble traversing the bistable geometry (Fig. S1A ) also depicts a minimum energy well required for bistability. In our representation the bubble in the lower lobe represents state 0 and the bubble in the upper lobe represents state 1. A toggle event occurs when a bubble arriving from the input flips the state of the device from 0 to 1 and vice versa. The device holds a single bubble indefinitely until another toggle signal arrives at the inlet $\mathrm{T}$ junction. Though the incoming bubbles are much longer than the Rayleigh-Plateau criteria for breakup $(l / \pi w>1$ where $l$ is length of the bubble and $w$ is the width) at a $\mathrm{T}$ junction[18], presence of a bubble in the flip-flop ensures the bubble travels to a single lobe without breakup (see bifurcation diagram Fig. S1B). The interconnecting channel allows flow between the two lobes, necessary to dislodge a trapped bubble once a toggle bubble arrives. The repeatability of this device is demonstrated via plotting a signal trace for the 
device for a period of 12 seconds (Fig. S1C). Bistability also allows us to construct sequential circuits including counters and memory arrays. We demonstrate a two-bit cascaded ripple counter by connecting toggle flip-flops in series (Fig. 3-2 H).

\subsection{On-demand bubble generator}

To provide an electronic interface to bubble logic devices we developed a thermal electrobubble modulator (Fig. 3-3) capable of generating bubbles on demand synchronized to an electric pulse. Methods for high-frequency continuous production of mono-disperse micro bubbles and droplets in microfluidic devices have been extensively studied $[23,9,26]$. Electro-generation of on-demand single aqueous droplets[13] requires high on-chip electric fields of the order of $\sim 1 \mathrm{kV}$. Our thermal electro-bubble generator uses an integrated microheater and modified flow-focusing geometry (Fig. 3-3), operating at low voltages (21V). For the case of pressure-driven flow (Fig. 3-3 A), a static force balance exists at the airwater interface with the pressure force being balanced by capillary and viscous stresses at the interface. This static balance can be written as $\Delta P+\tau_{v}=C a^{-1} k[26]$ where $\Delta P$ is the difference in pressure, $\tau_{v}$ is the viscous stress, $k$ represents the mean curvature, and $C a=\mu U / \sigma$ is the Capillary number. An applied temperature pulse reduces the surface tension $\sigma$ at the air-water interface. This lowers the curvature force and the pressure differential across the interface, allowing a gas filament to penetrate the liquid (Fig. 3-3 D), which breaks to form a single bubble (Fig. 3-3 E).

\subsection{Cascadability and feedback}

Usefulness of any logic family depends on cascadability to implement an arbitrary Boolean logic function as a sequence of logic gates. For implementation of a complex close-loop control, feedback is necessary. To show cascading and feedback we demonstrate a tunable microfluidic ring oscillator (Fig. 3-4A-B), as is common for characterization of any new logic family. The oscillator consists of three identical AND gates connected via three identical 

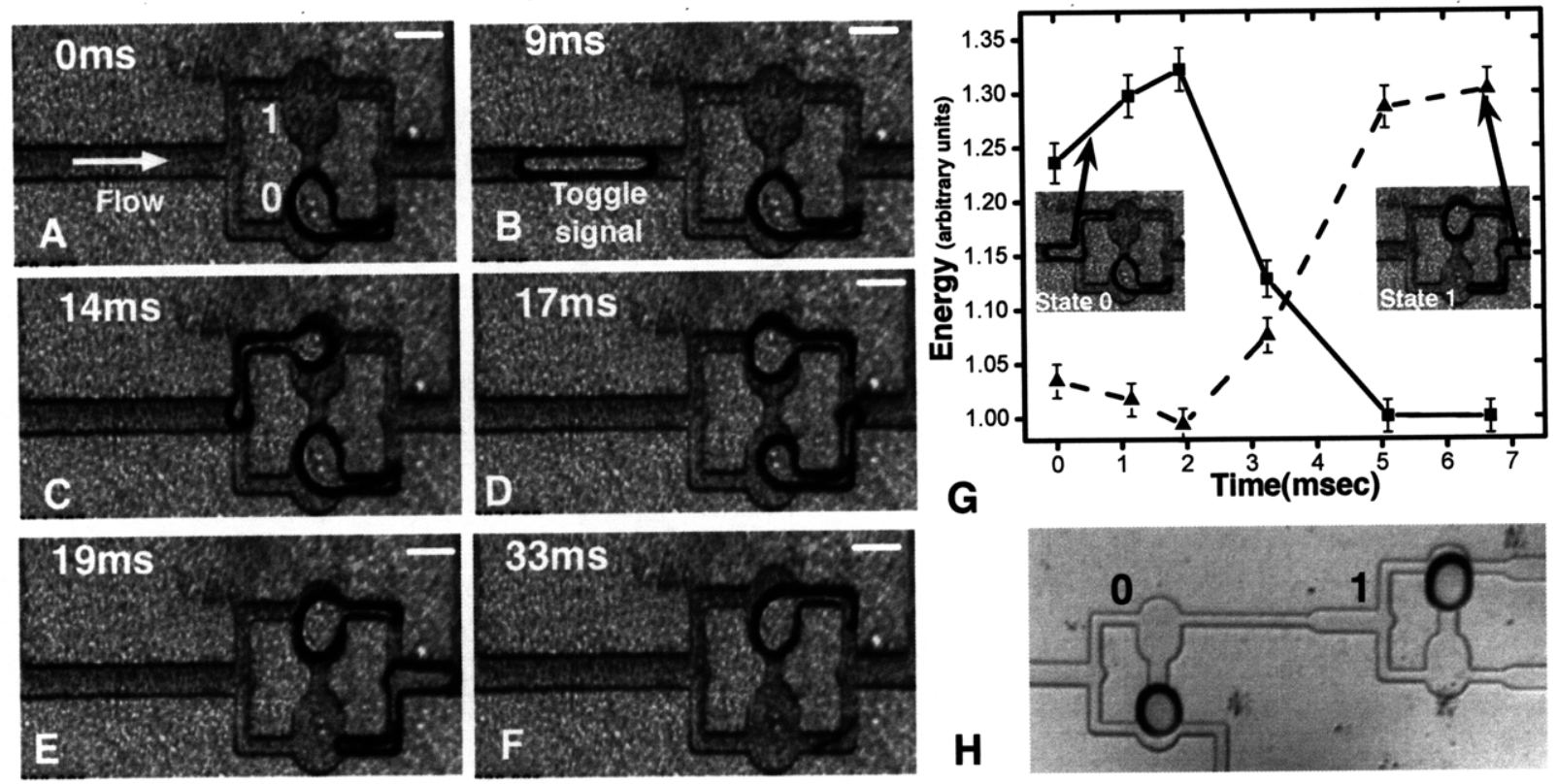

Figure 3-2: Bistability (A) Toggle flip-flop with one bit memory. The device stores a bubble (A) indefinitely against a constant flow ( $Q=0.25 \mathrm{\mu l} / \mathrm{sec}$ water with $2 \% \mathrm{w} / \mathrm{w}$ Tween 20 , driven by a syringe pump, Kent Scientific) in an energy well until a toggle signal arrives (B). The stored bubble is released from the trap while the arriving bubble is simultaneously captured in the opposing lobe, hence toggling the state of the device (C-F). The switching time for the device is $8 \mathrm{~ms}$ with an update rate of $30 \mathrm{~Hz}$. The capillary number $\mathrm{Ca}$ for given flow rate is 0.013 . The device consists of a planar geometry (channel height $70 \mu \mathrm{m}$ ) with two lobes $(200 \mu \mathrm{m}$ wide, $300 \mu \mathrm{m}$ long) connected via a pressure feedback channel (50 $\mu \mathrm{m}$ wide, $50 \mu \mathrm{m}$ long) and a $\mathrm{T}$ junction at inlet and outlet (50 $\mu \mathrm{m}$ wide). Scale bar 100 $\mu \mathrm{m}$. (B) Change in free surface energy of the bubble[1] depicting two energy minimas for a single flip-flop event. A toggle signal arrives from the right with an elongated shape with higher surface energy (solid line) while the bubble stored in the device occupies a lower energy state (dashed line). As the toggle bubble expands to find its minimum energy state in the upper lobe, pressure from the feedback and the input channel increases pushing the stored bubble into an elongated higher energy state into the outlet channel (dashed lines). This further stabilizes the toggle bubble by re-routing flow through the empty lobe. (C) Toggle flip-flops can be cascaded to build a ripple counter which updates its state on every 1 to 0 transition. 

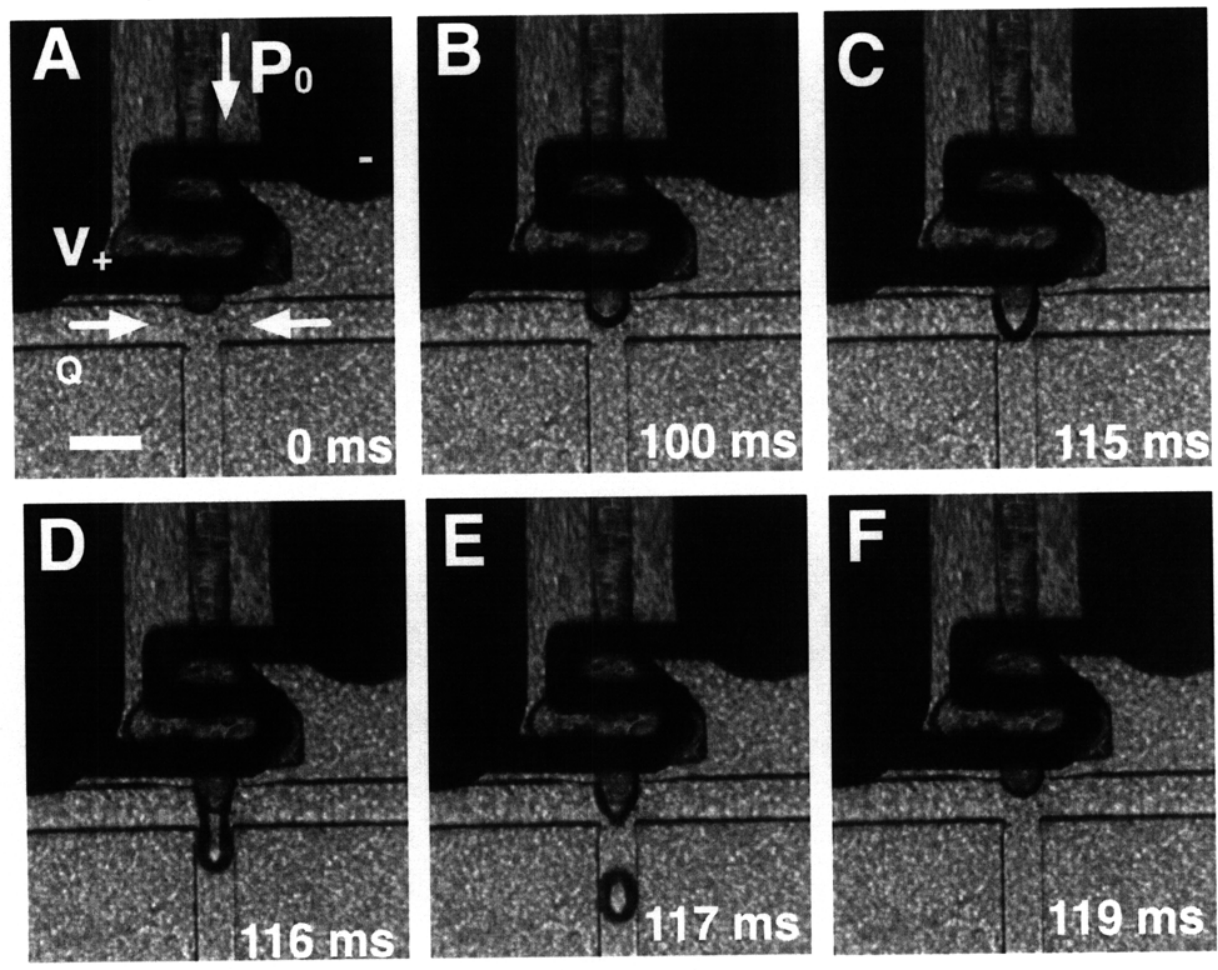

Figure 3-3: (A-F) Programmable on-demand electro-thermal bubble generator. The device consists of a $50 \mu \mathrm{m}$ wide platinum micro-heater $(R=95 \Omega, 200 \mathrm{~nm}$ thick platinum) followed by a $2 \mu \mathrm{m}$ silicon dioxide dielectric barrier. The heater is embedded below a modified, planar flow focusing geometry (channel height $70 \mu \mathrm{m}$, gas inlet orifice $70 \mu \mathrm{m}$ with a 400 $\mu \mathrm{m}$ expansion before the orifice) which pins the air-water interface making it stable. The device is driven by a constant flow $Q=0.83 \mathrm{\mu l} / \mathrm{sec}$ of water (with $2 \% \mathrm{w} / \mathrm{w}$ Tween 20 ) and a constant pressure supply $P_{0}=5 \mathrm{psi}$ of nitrogen. Series of photomicrographs depict a single bubble generated at a frequency of $1 \mathrm{~Hz}$. Scale bar is $100 \mu \mathrm{m}$. A 21V DC electric pulse is applied to the micro-heater in the first $100 \mathrm{~ms}$ of the sequence resulting in a growth period of the meniscus (A and C). Temperature induced change in surface tension of water lowers the capillary force allowing a gas filament to penetrate the water (D). The narrow entrained filament (E) breaks into a single drop via flow focusing [9]. The filament retracts back very quickly due to sharp curvature at the tip (E) and the interface is stabilized again $(\mathrm{F})$. Total time duration of the breakup process followed by a relaxation period is $25 \mathrm{~ms}$. 
delay lines in a ring structure (Fig. 3-4 A). Constant frequency $\mathrm{T}$ junction bubble generators act as inputs to the the ring oscillator providing a constant stream of bubbles. A bubble propagating from the ring delay line (Fig. 3-4 A, white arrow), increases the resistance of the outgoing channel when it arrives at one of the three AND gates. This generating a pressure pulse which launches another bubble in the delay line as a response. Cascaded switching of three AND gates in a ring structure achieves the function of an oscillator. The oscillation frequency of this device can be written as $f \propto 1 /\left[3\left(l / v+\tau_{d}\right)\right]$ where $f$ is the oscillator frequency, $l$ is the length of delay line, $v$ is the mean velocity of the bubble traveling in the delay line and $\tau_{d}$ is the propagation delay of the AND gate. The frequency of oscillations can be tuned, which is shown experimentally by increasing the flow rate of the continuos phase (Fig. 3-4 B).

For digital systems, where information is encoded in a timed sequence of bits, ability to synchronize two independent data streams is important for scalability. In an analogous manner, arrival time of a bubble at a logic gate governs the behavior of a bubble logic device. We demonstrate synchronization of two independently generated input bubble streams via a passive fluidic geometry. Such a timing restoration is also critical for on-chip passive droplet coalescence, which require two drops to arrive at a junction in a synchronized manner. We achieve timing restoration via a planar fluidic resistance ladder network. The geometry (Fig. 3-4C) includes inter-connecting fluid channels (continuous phase flow resistance $r$ ) between two data carrying channels (continuous phase flow resistance $R$, where $r>R$ (Fig. S2A-B)). A single bubble traversing the ladder geometry is slowed down due to presence of an alternate path (via inter-connecting channels) for the fluid behind the bubble. The total drop in velocity scales with number of inter-connecting channels. When both bubbles are present in the network simultaneously, inter-connecting channels between the two drops allow for net flow from channel with the leading bubble to the one with the lagging bubble. This relative velocity gradient allows for synchronization (Fig. S2C-D). Once the bubbles are synchronized in the network, the bubble configuration is symmetric and hence both bubbles travel at the same velocity in a synchronized manner. 

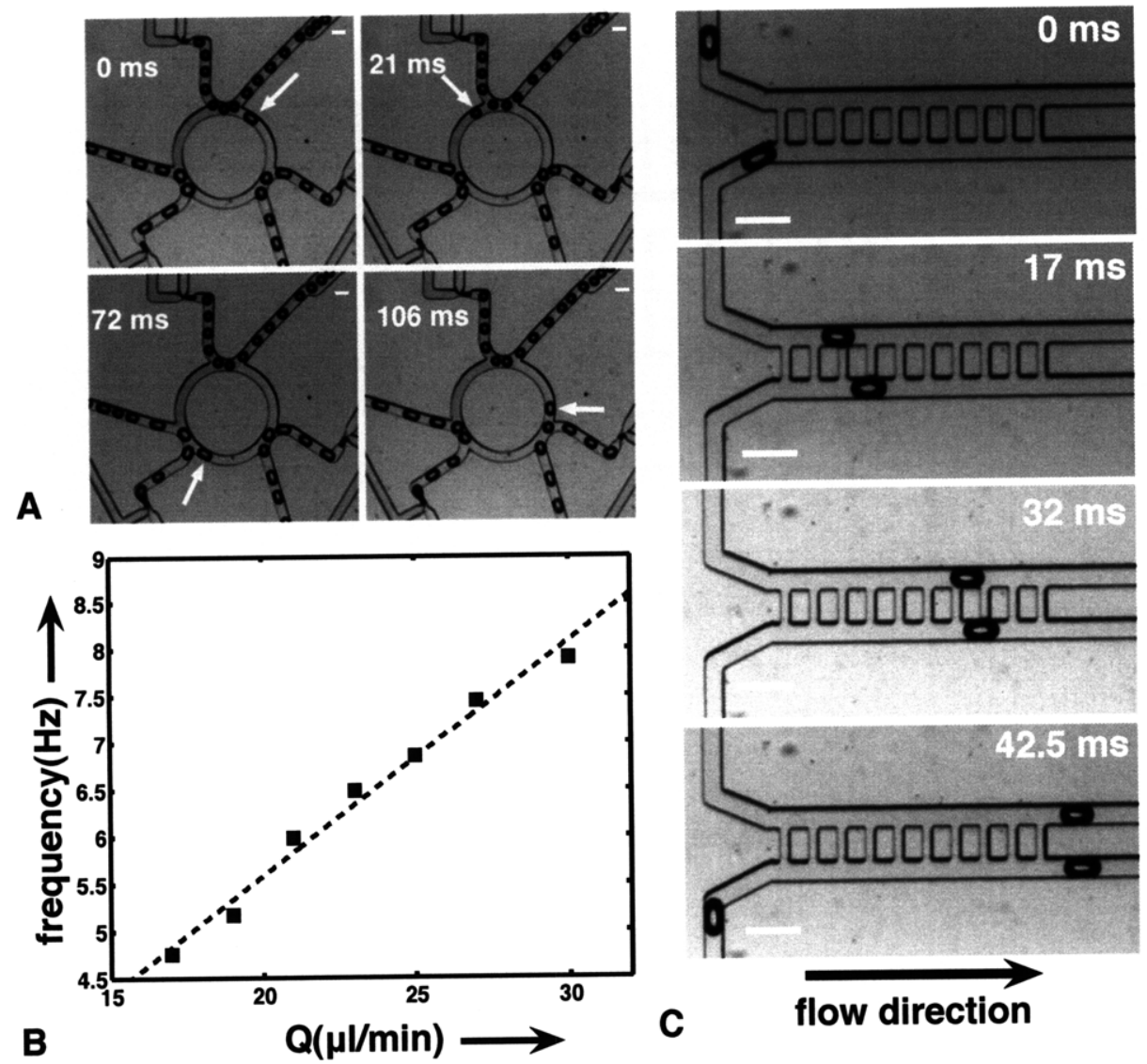

Figure 3-4: Feedback, cascadability and synchronization (A) Three AND gates are connected in a ring configuration via three delay lines to form a ring oscillator depicting feedback and cascadability. Three input ports are driven by $\mathrm{T}$ junction bubble generators with a constant flow (water $2 \% \mathrm{w} / \mathrm{w}$ Tween 20) from syringe pumps (Kent Scientific) and a constant pressure nitrogen line. A bubble in the delay line arrives at an AND gate causing a sharp increase in the outflow resistance of the gate and hence resulting in a release of another bubble in the delay line. White arrow in each frame marks bubbles traversing counter-clockwise in the ring. The frequency of oscillation for the device operated at $Q$ $=0.5 \mathrm{\mu l} / \mathrm{sec}, P_{0}=0.8 \mathrm{psi}$ is $7.9 \mathrm{~Hz}$. The propagation delay for the AND gate is $\sim 10 \mathrm{~ms}$ at a flow rate of $Q=0.5 \mathrm{\mu l} / \mathrm{sec}$. The $C a$ number for the device is 0.03 . Scale bar 100 $\mu \mathrm{m}$. (B) Dependence of the oscillation frequency on the flow rate $Q$, with a linear fit to the data. (C) A passive bubble synchronizer for timing restoration. The planar device (channel height $70 \mu \mathrm{m}$ ) consists of a fluidic ladder network with two (50 $\mathrm{\mu m}$ wide) parallel channels with equal flow (driven by constant flow syringe pump, Kent Scientific) and 10 interconnected channels ( $25 \mu \mathrm{m}$ wide, $100 \mu \mathrm{m}$ long, $75 \mu \mathrm{m}$ pitch). A timing restoration of $\sim 10 \mathrm{~ms}$ is achieved between two arriving bubbles over a span of $\sim 40 \mathrm{~ms}$ at a flow rate of $Q=0.5 \mathrm{\mu l} / \mathrm{sec}$. 


\subsection{Conclusion}

The device physics for bubble logic is governed by physical fluid dynamics of bubbles in static microchannel geometries with no moving parts. Thus bubble logic chips can be fabricated in a wide variety of materials including silicon and glass, allowing us to port reaction chemistries unsuitable for PDMS channels. Bubble logic devices operate at low Reynolds and Capillary numbers where surface tension and viscous forces are dominant compared to inertial forces, thus further reduction in size is feasible with faster switching times. The device mechanisms do not depend on non-Newtonian fluid properties, hence matching dimensionless flow parameters will allow bubble logic circuits to be designed using different fluids, eg. water droplets in oil and oil droplets in water.

The universal logic gates, toggle flip-flop, ripple counter, synchronizer, ring oscillator and electro-bubble modulator presented in this paper exhibit nonlinearity, bistability, gain, synchronization, cascading, feedback and signal encoding. Having shown the required properties of a scalable logic family, they can be used to create complex microfluidic circuits capable of performing arbitrary fluid process control and computation in an integrated fashion. This can reduce the size, cost and complexity of current microfluidic platforms, and make possible the development of very large scale microfluidic reactors for use in areas including combinatorial chemistry and drug discovery. Long term measurements on droplets in segmented flow micro-reactors require a programmable bistable trap for holding drops for arbitrary periods of time. Toggle flip-flop gate presented here can be used as a passive, reusable trap for the same. Bubble synchronizer presented here can also be used to passively remove any skew in arrival timings of droplets at a junction, necessary for onchip droplet coalescence. The ability to generate bubbles on demand provides a mechanism to encode information and thus program microfluidic bubble circuits. These bubble logic processors, where a bit of information can also carry a chemical payload, merge chemistry and computation. 


\section{Bibliography}

[1] Surface energy for a bubble trapped in a channel was evaluated from photomicrographs captured by a high speed camera (Phantom V4.0, Vision research). The grayscale images from the camera were digitized and a threshold filter was applied. An edge detector was used to find the bubbles and the perimeter of each blob was calculated using in Matlab.

[2] Frederick K. Balagadde, Lingchong You, Carl L. Hansen, Frances H. Arnold, and Stephen R. Quake. Long-term monitoring of bacteria undergoing programmed population control in a microchemostat. Science, 309(5731):137-140, 2005.

[3] Charles A. Belsterling. Fluidic Systems Design. Wiley Interscience, 1971.

[4] F. P. Bretherton. The motion of long bubbles in tubes. Journal of Fluid Mechanics, 10:166-188, 1961.

[5] Hsu Chang. Magnetic Bubble Logic: Integrated-circuit magnetics for digital storage and processing. IEEE Press, 1975.

[6] Arthur Conway. A Guide to Fluidics. Macdonald and Co., 1972.

[7] Galder Cristobal, Jean-Philippe Benoit, Mathieu Joanicot, and Armand Ajdari. Microfluidic bypass for efficient passive regulation of droplet traffic at a junction. Applied Physics Letters, 89:034104, 2006.

[8] Edward Fredkin and Tommaso Toffoli. Conservative logic. International Journal of Theoretical Physics, 21:219-253, 1982. 
[9] Pitor Garstecki, Irina Gitlin, Willow Diluzio, Geroge M. Whitesides, Eugenia Kumacheva, and Howard A. Stone. Formation of monodisperse bubbles in a microfluidic flow-focusing device. Applied Physics Letters, 85(13):2649-2651, 2004.

[10] Peter R. C. Gascoyne, Jody V. Vykoukal, Jon A. Schwartz, Thomas J. Anderson, Daynene M. Vykoukal, K. Wayne Current, Charles McConaghy, Frederick F. Beckera, and Craig Andrews. Dielectrophoresis-based programmable fluidic processors. Lab on Chip, 4(4):299-309, July 2004.

[11] Alex Groisman, Markus Enzelberger, and Stephen R. Quake. Microfluidic memory and control devices. Science, 300(5621):955 - 958, 2003.

[12] Alex Groisman and Stephen R. Quake. A microfluidic rectifier: Anisotropic flow resistance at low reynolds numbers. Physics Review Letters, 92(9):094301, 2004.

[13] Mingyan He, Jason S. Kuo, and Daniel T. Chiu. Electro-generation of single femtoliterand picoliter-volume aqueous droplets in microfluidic systems. Applied Physics Letters, 87:031916(1)-3, 2005.

[14] Klavs Jensen and Abraham Lee. The science \& applications of droplets in microfluidic devices. Lab on Chip, 4(4):31-32, 2004.

[15] Mathieu Joanicot and Armand Ajdari. Droplet control for microfluidics. Science, $309(5736): 887-888,2005$.

[16] Fabien Jousse, Guoping Lian, Ruth Janes, and John Melrose. Compact model for multi-phase liquid-liquid flows in micro-fluidic devices. Lab on a Chip, 5:646-656, 2005.

[17] Chung Cheng Lee, Guodong Sui, Arkadij Elizarov, Chengyi Jenny Shu, Young-Shik Shin, Alek N. Dooley, Jiang Huang, Antoine Daridon, Paul Wyatt, David Stout, Hartmuth C. Kolb, Owen N. Witte, Nagichettiar Satyamurthy, James R. Heath, Michael E. Phelps, Stephen R. Quake, and Hsian-Rong Tseng. Multistep synthesis of a radiolabeled imaging probe using integrated microfluidics. Science, 310:1793-1796, 2005. 
[18] D.R. Link, S.L. Anna, D.A. Weitz, and H.A. Stone. Geometrically mediated breakup of drops in microfluidic. Physical Review Letters, 92(5):054503, 2004.

[19] D.R. Link, Erwan Grasland-Mongrain, Agnes Duri, Flavie Sarrazin, Zhengdong Chang, Galder Cristobal, Manuel Marquez, and David .A. Weitz. Electric control of droplets in microfluidic devices. Angew. Chem. Int. Ed., 45:2556-2560, 2006.

[20] Chang W. Park. Influence of soluble surfactants on the motion of a finite bubble in a capillary tube. Physics of Fluids, 4(11):2335-2347, 1992.

[21] Yung-Chieh Tan, Jeffrey S. Fisher, Alan I. Lee, Vittorio Cristini, and Abraham Phillip Lee. Design of microfluidic channel geometries for teh control of droplet volume, chemical concentration and sorting. Lab on Chip, 4:292-298, 2004.

[22] Todd Thorsen, Sebastian J. Maerkl, and Stephen Quake. Microfluidic large-scale integration. Science, 298(5593):580-584, 2002.

[23] Todd Thorsen, Richard W. Roberts, Frances H. Arnold, and Stephen R. Quake. Dynamic pattern formation in a vesicle-generating microfluidic device. Physics Review Letters, 86(18):4163-4166, April 2001.

[24] Marc A. Unger, Hou-Pu Chou, Todd Thorsen, Axel Scherer, and Stephen R. Quake. Monolithic microfabricated valves and pumps by multilayer soft lithography. Science, 288(5463):113-116, 2000.

[25] Tor Vestad, David W.M. Marr, and Toshinori Munakata. Flow resistance for microfluidic logic operations. Applied Physics letters, 84:5074-5075, 2004.

[26] Thomas Ward, Magalie Faivre, Manouk Abkarian, and Howard Stone. Microfluidic flow focusing:drop size and scaling in pressure versus flow-rate-driven pumping. Electrophoresis, 26:3716-3724, 2005.

[27] Harris Wong, C.J. Radke, and S. Morris. The motion of long bubbles in polygonal capillaries. part 2. drag, fluid and fluid flow. Journal of Fluid Mechanics, 292:95-110, 1995. 
[28] Bo Zheng, L. Spencer Roach, and Rustem F. Ismagilov. Screening of protein crystallization conditions on a microfluidic chip using nanoliter-size droplets. Journal of American Chemical Society, 125(37):11170- 11171, August 2003. 


\section{Chapter 4}

\section{Device Design Principles}

\subsection{Introduction}

Droplet microfluidics as a field has grown tremendously in the last few years. A significant amount of work has been done on continuous generation of droplets at high frequencies. Thus the common design for droplet or multi-phase microfluidic device consists of a droplet generator followed by a linear channel and a point of observation in the channel. For a lab-on-chip device to be truly universal and handle a variety of reactions (like multistep reactions, in-built selection criteria, routing and so forth), complex droplet processing operations are necessary. In the previous chapter we have proposed a family of modular devices which can be put together to form more complex droplet processing units. We also show that the set of devices can perform universal computation, thus any fluidic droplet processing circuit can be fabricated from a limited set of units.

In this chapter we cover in further details device design principles and reduced order models for devices described previously. We first review a droplet junction device, proposed and implemented by Ajdari et. al [2]. Further, we describe simple models for evaluating operating conditions for numerous devices proposed in our work. 

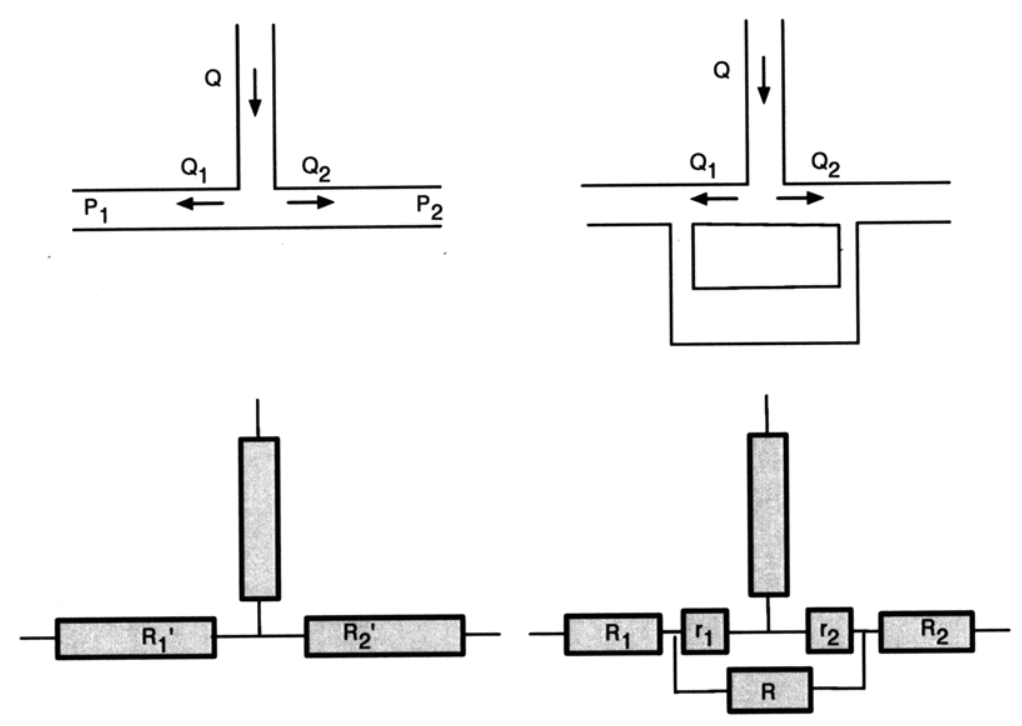

Figure 4-1: Schematic and reduced model representation of a spit channel junction and split channel junction with a bypass channel. The effective resistance is depicted to show the variation and non-linear behavior of the channel network as droplets pass through the same. The device shows a memory effect, where the past history of the droplets determine the future of the droplet arriving at the junction. Figure adapted from Ajdari et. al. [2]

\subsection{Droplets at a junction and bypass channels}

Ajdari et. al[2] considered a simple droplet circuit; a channel splitting into two units. Due to the discreet nature of droplets in such a geometry, a binary decision is made by droplets arriving at such a junction at low Capillary numbers $\left(C a<10^{-2}\right)$. Furthermore, the distribution of droplets in such a setting is primarily governed by the history of the device, that is distribution of droplets in the two split-lines govern the fate of the droplet just arriving at the junction point. Thus a dynamic memory like behavior is exhibited by the device. For numerous applications (for e.g. fan-out or a binary distribution network) it is desired that droplets be equally split amongst the output ports (equal in number). This behavior is not supported by a simple symmetric junction geometry that exhibits a dynamic (and fairly random at individual droplet level) behavior.

Ajdari et. al. proposed a simple bypass junction between the two output channels, providing a pressure communication channel between the two outlets. The operating mechanism using the bypass channel - ensures that once a droplet enters one junction, the flow rate of 
the second junction is higher and hence the second droplet is shuttled towards the second branch. This provides a passive means to distribute droplets in a junction geometry equally and in an alternative sequence. This can be quantitatively represented in a reduced order model for a geometry with and without the bypass channel following Ajdari et al. [2]. For input flow rate $Q(t)$ dividing into $Q_{1}(t)$ and $Q_{2}(t)=Q(t)-Q_{1}(t)$ and outlet pressure at both ends being equal, i.e. $P_{1}=P_{2}$

$$
\frac{Q_{1}-Q_{2}}{Q}(t)=\left(\frac{R_{2}^{\prime}-R_{1}^{\prime}}{R_{2}^{\prime}+R_{1}^{\prime}}\right)(t)
$$

where $R_{i}^{\prime}(t)=R_{i}^{0}+n_{i}(t) \delta r$, where $n_{i}$ is the number of droplets in a given channel and $\delta r$ is the increase in flow resistance due to a droplet in a channel. The junction to which an arriving droplet travels is given by the sign of $Q_{1}-Q_{2}$ which is dependent on $R_{2}^{\prime}-R_{1}^{\prime}$ and hence on the history of droplets which are in the two branches. Once a bypass junction is included in this simple model, and for $R_{b p}<<R_{1}, R_{2}$ we have

$$
\frac{Q_{1}-Q_{2}}{Q}=\frac{\left(r_{2}+r_{1}\right)}{r_{1}+r_{2}+R_{b p}}\left(\frac{r_{2}-r_{1}}{r_{2}+r_{1}}\right)+\frac{R_{b p}}{r_{1}+r_{2}+R_{b p}} \times\left[\frac{R_{2}-R 1}{R_{1}+R_{2}}+\frac{2\left(P_{2}-P_{1}\right)}{Q\left(R_{1}+R_{2}\right)}\right]
$$

Thus sign of $Q_{1}-Q_{2}$ is sensitive to $r_{2}-r_{1}$ and insensitive to $R_{2}-R_{1}$. Hence the droplets follow the alternate route where $r_{2}$ and $r_{1}$ alternate in value because of the direction the droplet previous to the one at the junction takes. This design was built and tested for this parameter regime by Ajdari et. al. [2].

\subsection{Microfluidic Logic Gates - AND/NOT gate}

Here we consider the AND/NOT Boolean logic gate implemented in two-phase microfluidic circuits. Since "Bubble Logic" is a conservative logic scheme, bits can not be created or destroyed in the channels at will (which is the case for electronic logic schemes). Thus any non-conservative logic gate, for e.g. a AND gate needs to be integrated with another gate to suffice for conservative property of the bits. This also provides multiple logic operations in a single step performed in a gate, outputs of which can be utilized individually. 

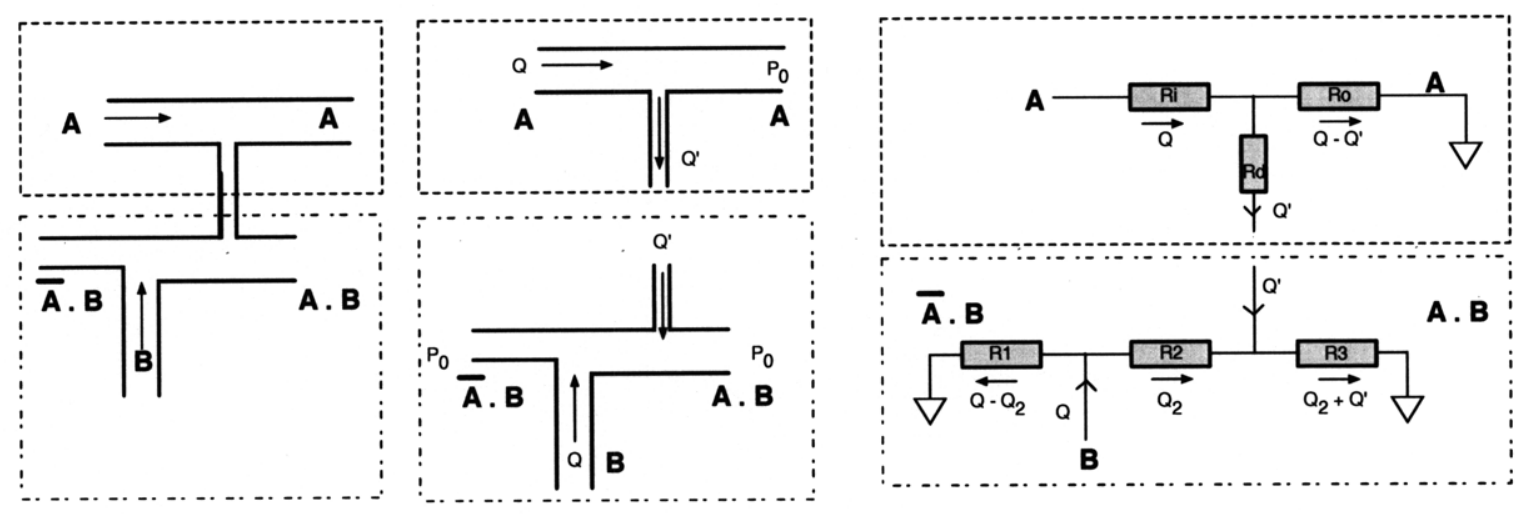

Figure 4-2: Schematic representation of AND/NOT gate geometry. Reduced order model of the logic gate is developed using non-linear resistor network represented as $R_{i}$ for hydraulic resistance of a channel, $Q_{i}$ for flow rate in a channel and $P_{0}$ for outlet pressure. Schematic representation of the truth table for $\operatorname{NOT}(A) \cdot B$ and $A . B$ is shown below.

Here we consider a reduced order model of the AND/NOT gate geometry presented in the previous chapter, and consider the dependence of channel geometries to gate performance. The device consists of two inlet ports and three outlet ports. With inlet ports marked A and B with constant input flow rate $Q$ and outlet ports marked with NOT(A).B and A.B at a constant pressure given by $P_{0}$. The device consists of an asymmetric $\mathrm{T}$ junction (marked with dash-dot in figure 4-2) with different hydraulic resistance $R_{1}$ and $R_{2}$ where $R_{1}>R_{2}$. Furthermore, an inlet port joins the $\mathrm{T}$ junction dumping a controlled fluid flow $Q^{\prime}$ (marked with dash lines in fig 4-2) at one of the outputs of the T junction. The device is thus characterized by hydraulic resistance $R_{1}, R_{2}, R_{3}$ for bottom $\mathrm{T}$ junction and $R_{i}, R_{d}, R_{o}$ for the top inlet. The schematic below shows the two cases where $A=1$ and $A=0$, as it evolves over time $t$. For the asymmetric T junction, the following conditions must be met during device operation; Case 1) for $A=1$, i.e. $Q^{\prime}<Q_{c r i t}$

$$
Q_{2}>0.5 Q
$$

Case 2) for $A=0$, i.e. $Q^{\prime}>Q_{c r i t}$

$$
Q_{2}<0.5 Q
$$


From Kirchhoff's laws for circuit geometry in figure 4-2

$$
Q_{2}=\left[\frac{R_{1} Q-R_{3} Q^{\prime}}{R_{1}+R_{2}+R_{3}}\right]
$$

or

$$
\frac{Q_{2}}{Q}=\left[\frac{R_{1}-R_{3} Q^{\prime} / Q}{R_{1}+R_{2}+R_{3}}\right]
$$

Normalizing with respect to $R_{3}$, we obtain the following condition that needs to be satisfied by the network for $A=1$

$$
\bar{R}_{1}-\bar{R}_{2}>\left(\frac{2 Q^{\prime}}{Q}+1\right)
$$

We now consider the top inlet flow geometry with bypass channel width $w_{d}$ and inlet channel width $w$ and height of all channels defined as $b$. A droplet or a bubble entering the channel from inlet marked $\mathrm{A}$, reduces the inlet flow $Q^{\prime}$ entering the asymmetric $\mathrm{T}$ junction for a short duration of time (the time it takes to pass the droplet through top channel). This results in $Q^{\prime}$ dropping below the $Q_{\text {critical }}$ (defined above), thus turning the gate ON for $A=1$. When no droplet or bubble is present in inlet channel marked $A$, the logic gate is OFF with $Q^{\prime}>Q_{\text {critical }}$. For top geometry (marked with dashed lines in figure 4-2) with inlet resistance $R_{i}$, outlet resistance $R_{o}$ and resistance of bypass channel $R_{d}$, we can write the following pressure balance equations (for duration of time a droplet blocks the junction)

$$
\begin{gathered}
Q_{o}=Q-Q^{\prime} \\
P_{d}+R_{d} Q^{\prime}+\gamma\left[\frac{1}{b}+\frac{1}{w_{d}}\right]=P_{o}+R_{o} Q_{o}+\gamma\left[\frac{1}{b}+\frac{1}{w}\right]
\end{gathered}
$$

Replacing for $Q_{o}$ and assuming $P_{o}=0$, we obtain

$$
P_{d}=R_{o}\left(Q-Q^{\prime}\right)-R_{d} Q^{\prime}+\gamma\left[\frac{1}{w}+\frac{1}{w_{d}}\right]
$$

Connecting the top and bottom parts by equating the pressure and net flow through the bypass junction, we can evaluate the value of $P_{d}$ as

$$
P_{d}=Q\left[\frac{\left(R_{1}+R_{2}\right)\left(R_{1}-R_{3} Q^{\prime} / Q\right)}{\left(R_{1}+R_{2}+R_{3}\right)}-R_{1}\right]
$$


Thus solving for $Q^{\prime}$ and $Q_{2}$

$$
Q^{\prime}=\frac{1}{\bar{R}}\left[R_{o}-R_{1}-\frac{\left(R_{1}+R_{2}\right) R_{1}}{\left(R_{1}+R_{2}+R_{3}\right)}\right] Q+\gamma\left[\frac{1}{w}-\frac{1}{w_{d}}\right]
$$

where $\bar{R}=\left(R_{d}+R_{o}\right)+R_{3}\left(R_{1}+R_{2}\right) /\left(R_{1}+R_{3}\right)$. Thus for $A=1$ (bubble or a droplet in inlet channel A), we obtain

$$
Q^{\prime}<\frac{Q}{2 R_{3}}\left(R_{1}-R_{2}-R_{3}\right)
$$

Simplifying with $R_{1}>>R_{2}$ and $R_{1}>>R_{3}$, we obtain

$$
\frac{Q_{2}-Q_{1}}{Q}=\left[\frac{R_{d}+5 R_{3}}{R_{d}+R_{o}+R_{3}}\right]+\left[\frac{R_{o}\left(R_{1}-2 R_{3}\right)}{R_{1}\left(R_{d}+R_{o}+R_{3}\right)}\right]+\frac{\gamma}{Q}\left[\frac{1}{w}-\frac{1}{w_{d}}\right]
$$

where the sign for $\left(Q_{2}-Q_{1}\right) / Q$ is sensitive to $R_{o}\left(R_{1}-2 R_{3}\right) / R_{1}\left(R_{d}+R_{o}+R_{3}\right)$ and $w-w_{d}$.

\subsection{Bistable one-bit memory}

A significant research effort in the field of droplet microfluidics has been devoted to high frequency generation of fluid micro-drops and bubbles in microfluidic geometries. Several groups have reported generation of continuous streams of droplets and bubbles at frequencies as high as $10 \mathrm{kHz}$. For any bio-chemical analysis on chip, production of fluid particles is followed by in-line measurement for characterization of processes being studied. For e.g. single cell analysis studies require in-line fluorescence measurement from individual droplets produced at high frequencies. Since these measurements are made on moving droplets, only a small time window for measurement $(t \sim d / v)$ is present; where $d$ is length of the drop and $v$ is velocity of the moving droplet. For high frequency droplet generation, this time is usually of the order of several $\sim m s$. Since measurement time window usually is directly correlated to analytical techniques employed and sensitivity of the process; a variable measuring slot is required. The bistable one-bit memory unit presented here provides such a device that allows for storage of a droplet or a bubble for a programmable duration of timefrom milliseconds to several minutes.

Bistable one-bit memory (toggle flip-flop) presented in previous chapter provides a pro- 


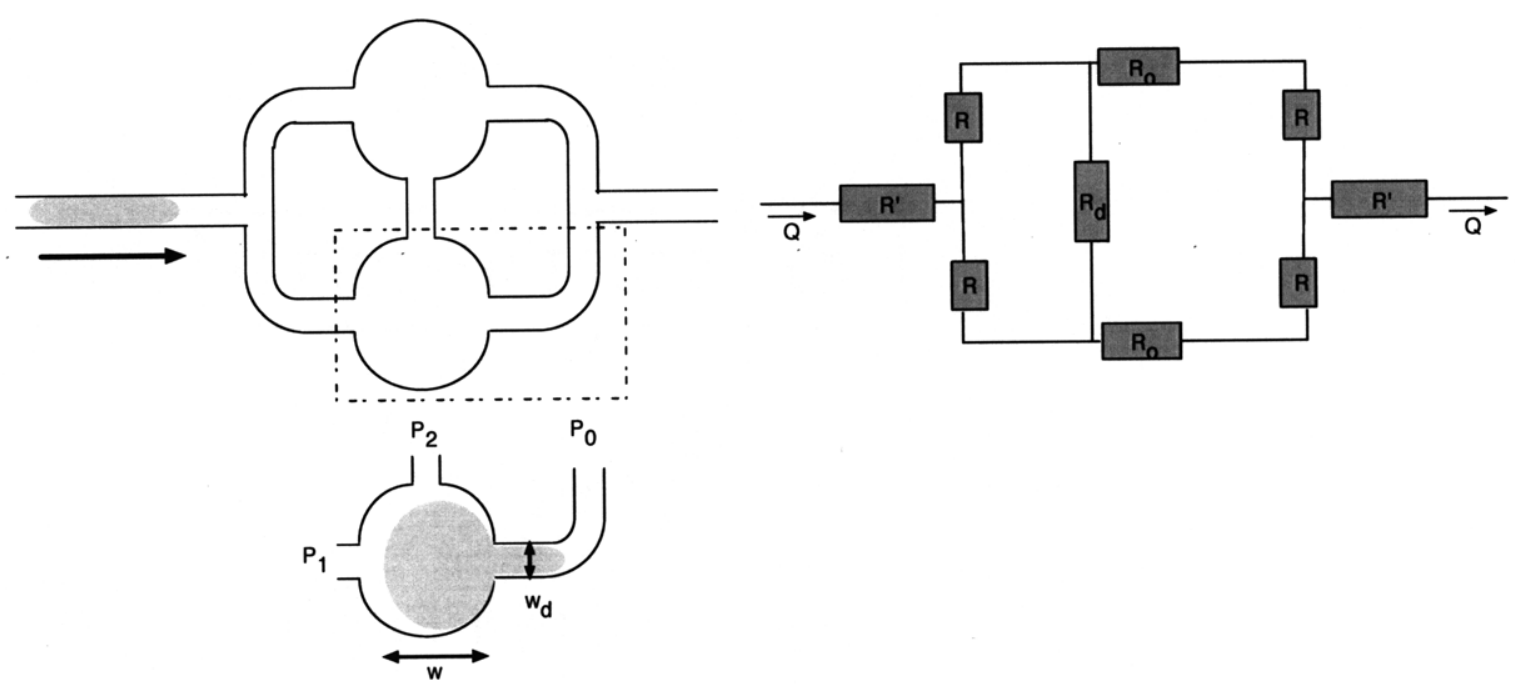

Figure 4-3: (A) Schematic of a bistable one-bit memory unit implemented in microfluidic bubble logic. The device is designed to store an arriving droplet until a toggle signal is applied to the inlet line. The maximum flow rate that can be applied to the device is given by $Q_{m} a x$. (B) A reduced order model for the device with a resistive network model is shown in schematic.

grammable one-bit of storage. The state of the device is represented as presence or absence of a bubble in upper or lower storage chamber (see figure 4-3). A droplet is stored until a toggle signal is applied to the device. Arrival of another droplet unlatches the stored unit while capturing the arriving droplet. Thus the state of the memory unit is flipped, until the next toggle signal. Figure above 4-3 shows a reduced order model of the device as a resistive network. The device consists of a single inlet and a single outlet, with a feedback bypass channel connecting the two storage units. When a single droplet is stored in the device geometry; flow resistance through the branch with a captured drop increases significantly. Thus almost entire flow $Q$ is routed through an alternate branch. For a liquid-liquid surface tension $\gamma$ and geometry described in figure 4-3 we can write the maximum clogging pressure for the droplet

$$
P_{\text {clogging }}=\gamma\left[\frac{1}{w_{d}}-\frac{1}{w}\right]
$$

given by the difference in curvature pressure due to squeezing of the droplet in a narrowing geometry. 
Pressure $P_{1}$ and $P_{2}$ at the two ports marked in figure 4-3 is given by

$$
P_{1}=\left(R^{\prime}+2 R+R_{0}\right) Q
$$

and

$$
P_{2}=\left(R^{\prime}+R+R_{0}\right) Q
$$

Thus the condition for maximum flow rate $Q_{\max }$ for which the stored droplet is stable is given by

$$
Q_{\max }=\frac{\gamma}{2 R^{\prime}+3 R+2 R_{0}}\left[\frac{1}{w_{d}}-\frac{1}{w}\right]
$$

Figure 4-4 also depicts the bifurcation diagram for one-bit flip-flop operated with varying volume or size of inlet bubble. The experiment consists of increasing the volume fraction of the bubble keeping a constant flow rate as input to the device. We plot the difference in volume of the output from the two storage compartments as a function of dimensionless size of the bubble, and find a bifurcation that occurs at a critical size of the input bubble. Thus bubbles smaller than the critical size $R_{\text {crit }}$ given size split into two sub-units while bubbles larger than $R_{\text {crit }}$ are stored in the flip-flop. Figure 4-4 C depicts a trace record of toggle and state of the flip-flop (blue and red curve). Furthermore, device operation is independent of the arrival frequency of the toggle signal.

\subsection{Synchronization in microfluidic circuits}

This part of the current chapter also appeared as a short paper titled "Synchronization in microfluidic circuits, Prakash and Gershenfeld, Proceedings of MicroTAS Oct 2007".

\subsubsection{Abstract}

In [4] we introduced Bubble logic, a new digital logic family implemented in two-phase microfluidics capable of universal computation. Bubble logic brings the logic, control and 

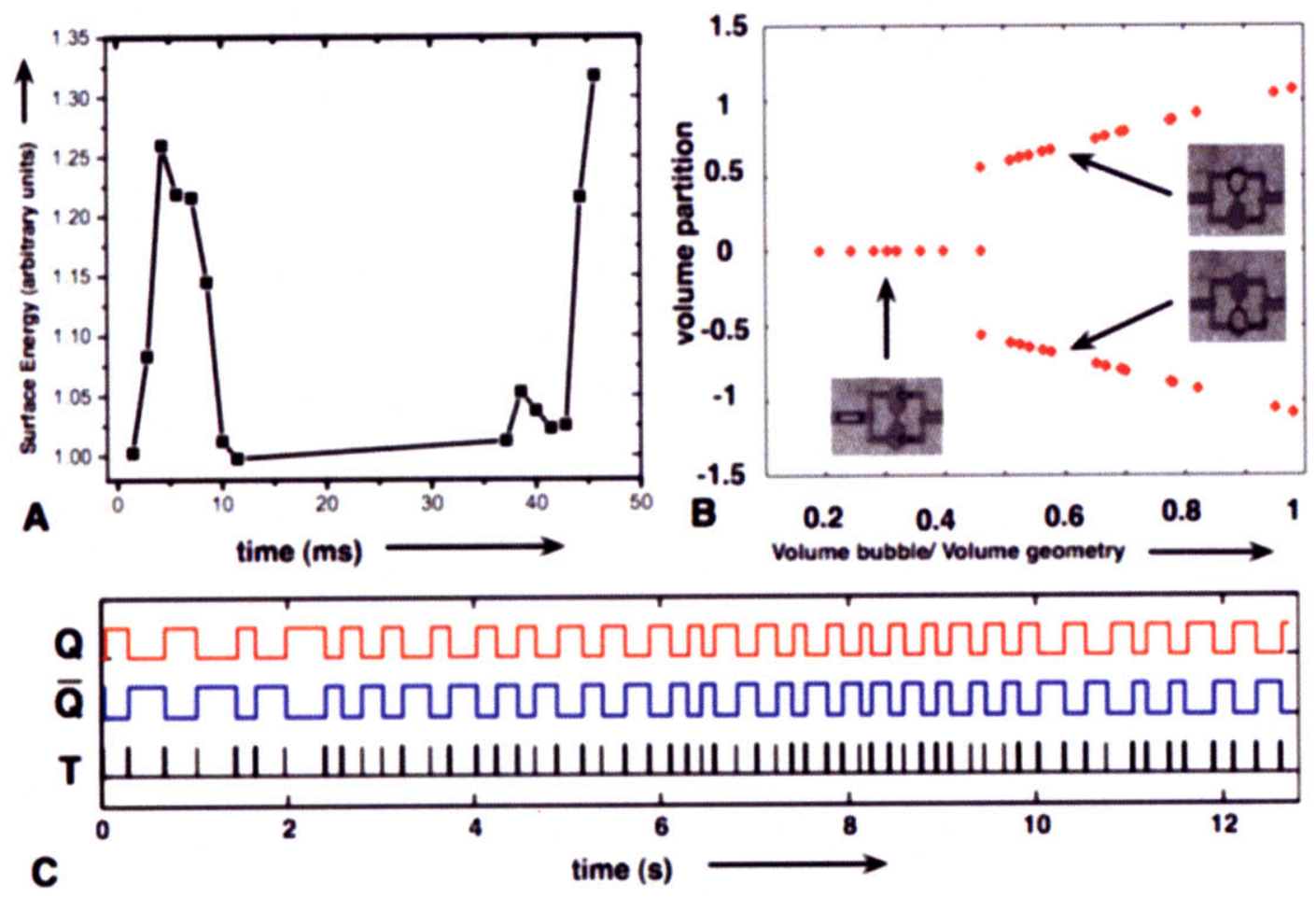

Figure 4-4: (A) Surface energy plot for a single bubble traversing the toggle flip-flop geometry vs. time. The large minima in the middle refers to the minimum energy well formed at the two lobes. (B) Bifurcation diagram for the toggle flip-flop geometry depicting a sudden transition from bubble breakup to the onset of bistablity. Plot of difference in bubble volume in the two lobes (volume partition of the bubble) is plotted against non-dimensional size of input bubble. All input bubbles considered in the dataset are longer than Rayleigh-Plateau breakup criteria for a $\mathrm{T}$ junction $(l / w>\pi, l$ is length and $w$ is width of the bubble). The bifurcation from equal bubble breakup to bistable mode occurs sharply. (C) To verify repeatability and stability of toggle flip-flop mechanism, a 12 second signal trace was recorded as light intensity. The device also showed nodependence on arrival frequency of bubbles at a junction. 
actuation of drops and bubbles on-chip, so bits can simultaneously represent and manipulate materials and information. For error correction in microfluidic circuits we introduced a novel ladder network geometry for passive synchronization of bubbles and drops. In this paper we quantify both experimentally and theoretically the performance of this timing restoration device. Our passive synchronization scheme provides a simple, scalable error correction mechanism for multiphase didgital microfluidic systems, with applications in microfluidic logic [4], droplet coalescence [1] and large scale integrated microfluidics.

\subsubsection{Introduction}

Current microfluidic technologies show a great promise to revolutionize analytical chemistry and biology, but still rely on lab full of external plumbing, valves and computers to operate. Very large scale integrated (VLSI) microfluidics requires scalable, on-chip control mechanisms for complex routing, storage and processing of reactants on a chip[3]. To this end, we introduced a digital Boolean logic family; Bubble Logic [4], which provides a modular process control mechanism for multi-phase microfluidic systems. Bubble logic uses nonlinear hydrodynamic interactions between bubbles flowing in microfluidic geometries to perform complex control operations for e.g. storage, counting etc., without any external macro scale elements like solenoids. For large scale error-free operation of bubble logic, timing errors between different bubble generators need to be corrected. Numerous authors have also reported the need for droplets to arrive in a synchronous manner [2] for error-free, on-chip droplet coalescence. Here we present experimental data and a lumped element non-linear resistor network model of our design, which passively synchronizes bubbles travelling in two microfluidic channels with no external control.

\subsubsection{Microfluidic Ladder Network synchronizer (LNS)}

Design of a LNS (Figure 4-5(A)) consists of two planar microfluidic channels (width=w2, height $=\mathrm{h}$ ) with two inlet ports marked 1 and 2 and two outlet ports marked 3 and 4 , interconnected by a number of higher resistance flow channels (length $\mathrm{L}$, width $=\mathrm{w} 3$ ). 

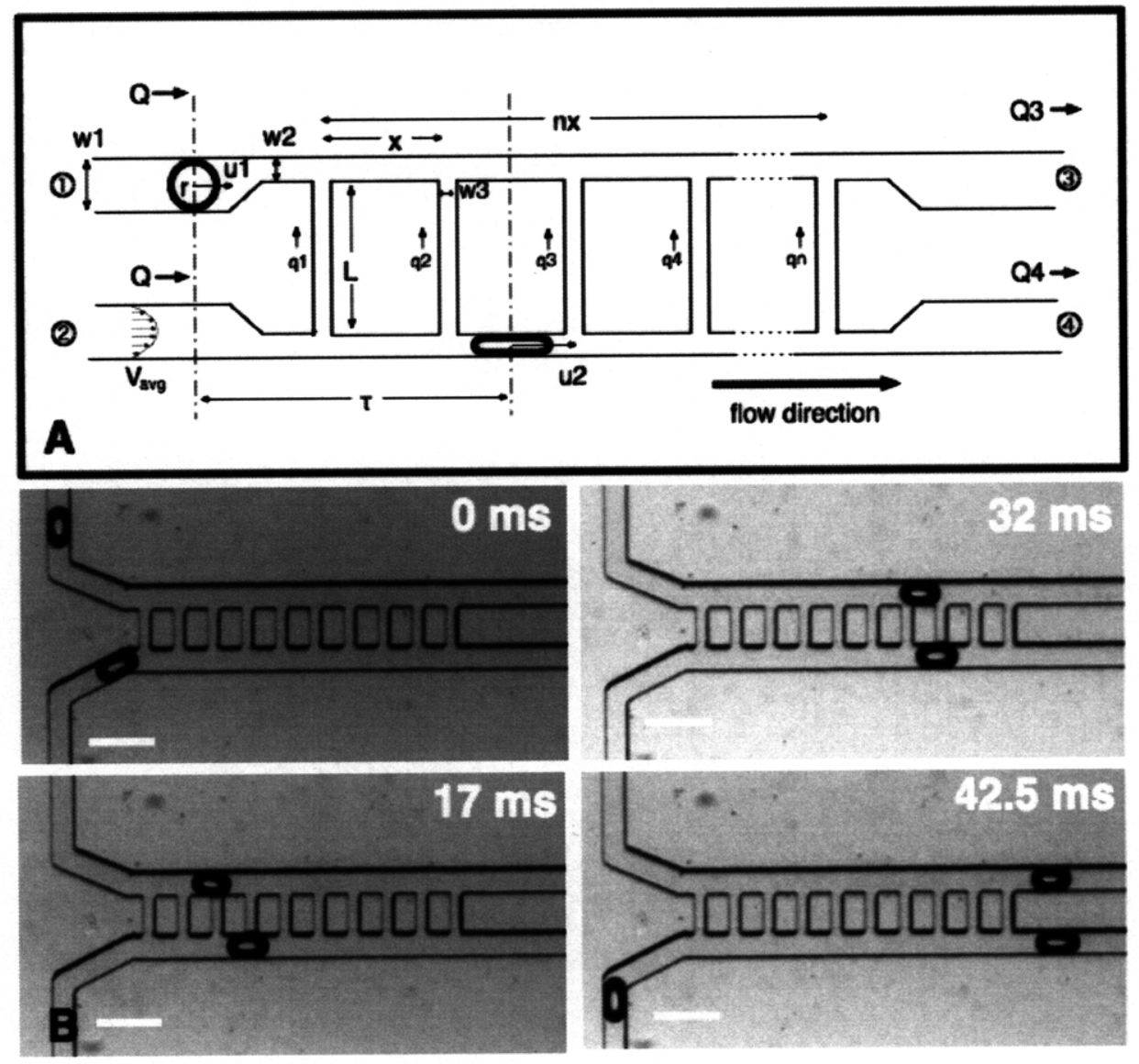

Figure 4-5: (A) Schematic of synchronization geometry. Bubbles are introduced into the channels at a constant frequency from left to right using a flow-focusing bubble generator operating at a constant flow-rate. (B) Passive synchronization of bubbles in top and bottom channels (width $=100 \mu \mathrm{m})$ with interconnected channels $(\mathrm{K}=10$, width $=50 \mu \mathrm{m})$ operating at a constant flow rate of $\mathrm{Qw}=0.5 \mu \mathrm{l} / \mathrm{sec}$. Timing restoration of $10 \mathrm{~ms}$ is achieved in $40 \mathrm{~ms}$. Scale bar $100 \mu \mathrm{m}$. 

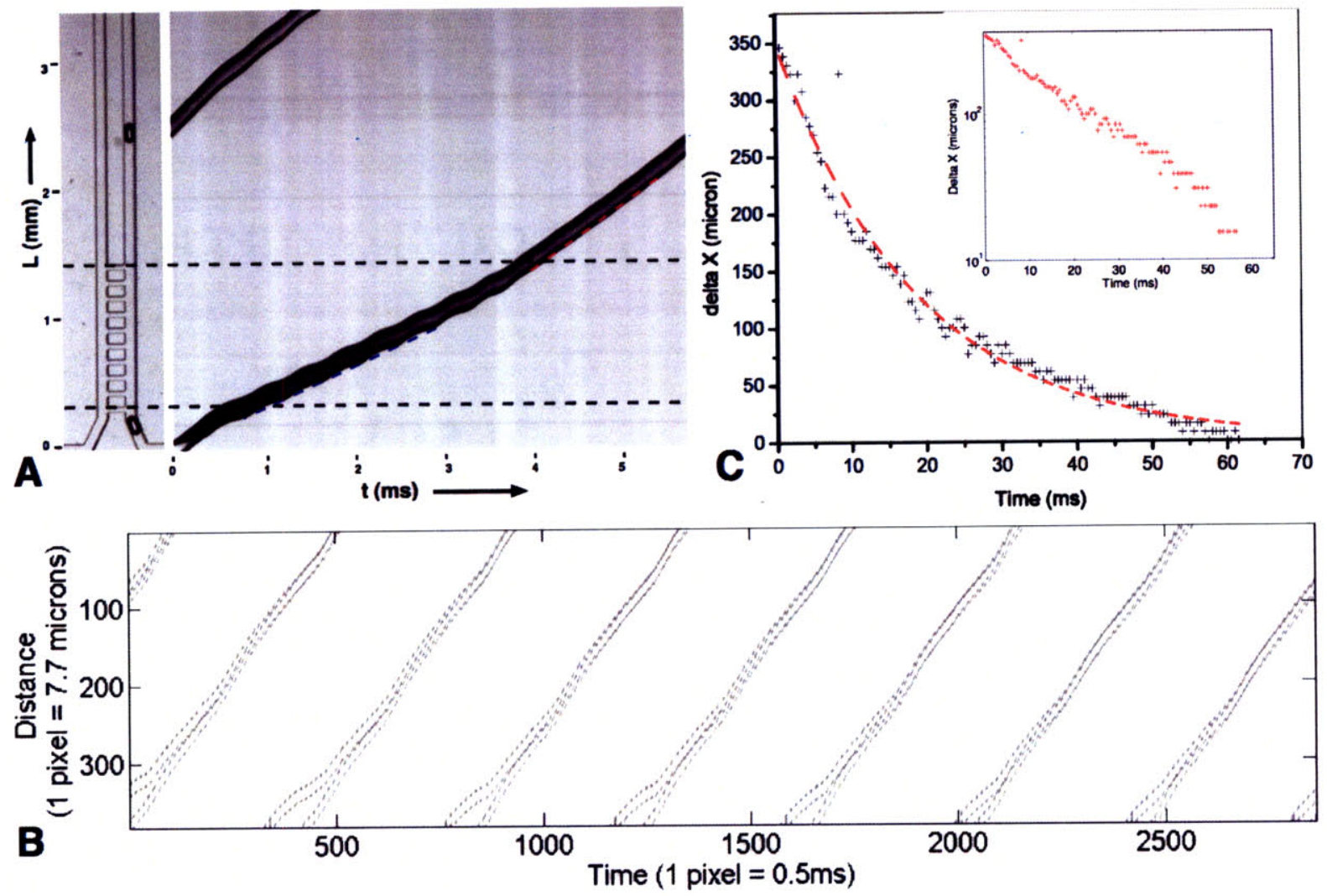

Figure 4-6: (A) Time series of a single bubble passing through a ladder network. The bubble slows down as it enters the inter-connected segment. Oscillations in the bubble velocity can be seen whenever the bubble crosses the interconnected vertical channel. (B) Dynamics of continuous synchronization at $5 \mathrm{~Hz}$. (C) Plot of experimental data of distance between the two bubbles vs time with an exponential fit. Inset depicts a log linear plot of decreasing distance with time.

Bubbles travel from left to right in the device geometry forced by a constant flow of continous phase from the inlet ports. The device operates on the principle of hydrodynamic coupling of flow as bubbles travel in the horizontal channels. The device operates passively with no external control and has no moving parts. This allows fabrication of the device in a large number of material sets, compatible with microfluidic processing.

A single bubble entering the synchronizer is slowed down as it travls from left to right. This is due to increase in fluidic resistance of the horizontal channel due to presence of a bubble and existence of an alternate low resistance path for the fluid behind the bubble. Due to 
the discrete nature of the synchronizer geometry, bubble velocity oscillates as it passes over the interconnected channels. When two bubbles enter the geometry with a time delay $\tau$, a net flow from the channel with leading bubble to the one with lagging bubble, creates a relative velocity gradient synchronizing the two bubbles. The operation of the device is depicted in a time series of micrographs in figure 4-5(B).

\subsubsection{Results and Discussion}

Figure 4-6 and 4-10 show synchronization dynamics of bubbles in a LNS device. Figure 4-6(A) shows a time trace obtained by high speed imaging (Phantom v4, $1000 \mathrm{fps}$ ) of a single nitrogen bubble in water (2\% Tween $20 \mathrm{wt}$.) travelling through the LNS geometry. The slope of the trajectory (bubble velocity) is constant in the three zones marked by dashed lines. The bubble velocity is reduced as it traverses the inter-connected channels. Periodic oscillations in bubble velocity are evident due to discrete resistive elements present in device. The device can operate in a continuous fashion, as shown in Figure 4-6(B) with LNS operating at $5 \mathrm{~Hz}$. Figure 4-6(C) shows a plot of horizontal distance between the two bubbles reducing with time, with an exponential fit to the data (dashed line). Inset depicts a log-linear plot of distance with time.

Consider a ladder network with two inputs and two outlet channels. The network is driven by a constant flow $Q$ with constant pressure $P_{o}$ at the outlet channel. Parallel channels are marked with resistance $R_{A i}$ and $R_{B i}$, and $R_{i}$ for bypass channels. For the results presented, we consider $R_{A i}=R_{B i}=R$ and $R_{i}=r$. The resistance due to a bubble in a channel is given by $R_{b}$. Normalizing flow resistance with respect to $R$; we obtain $\bar{r}=r / R, \bar{R} b=R b / R$. The length of inlet and outlet segments is given by $L_{1}$ while the pitch of interconnecting channels is defined as $L_{2}$ (see figure 4-7). $\mathrm{N}$ depicts the number of bypass channels in the network. For any non-linear ladder network, the following set of equations are satisfied at the nodes 

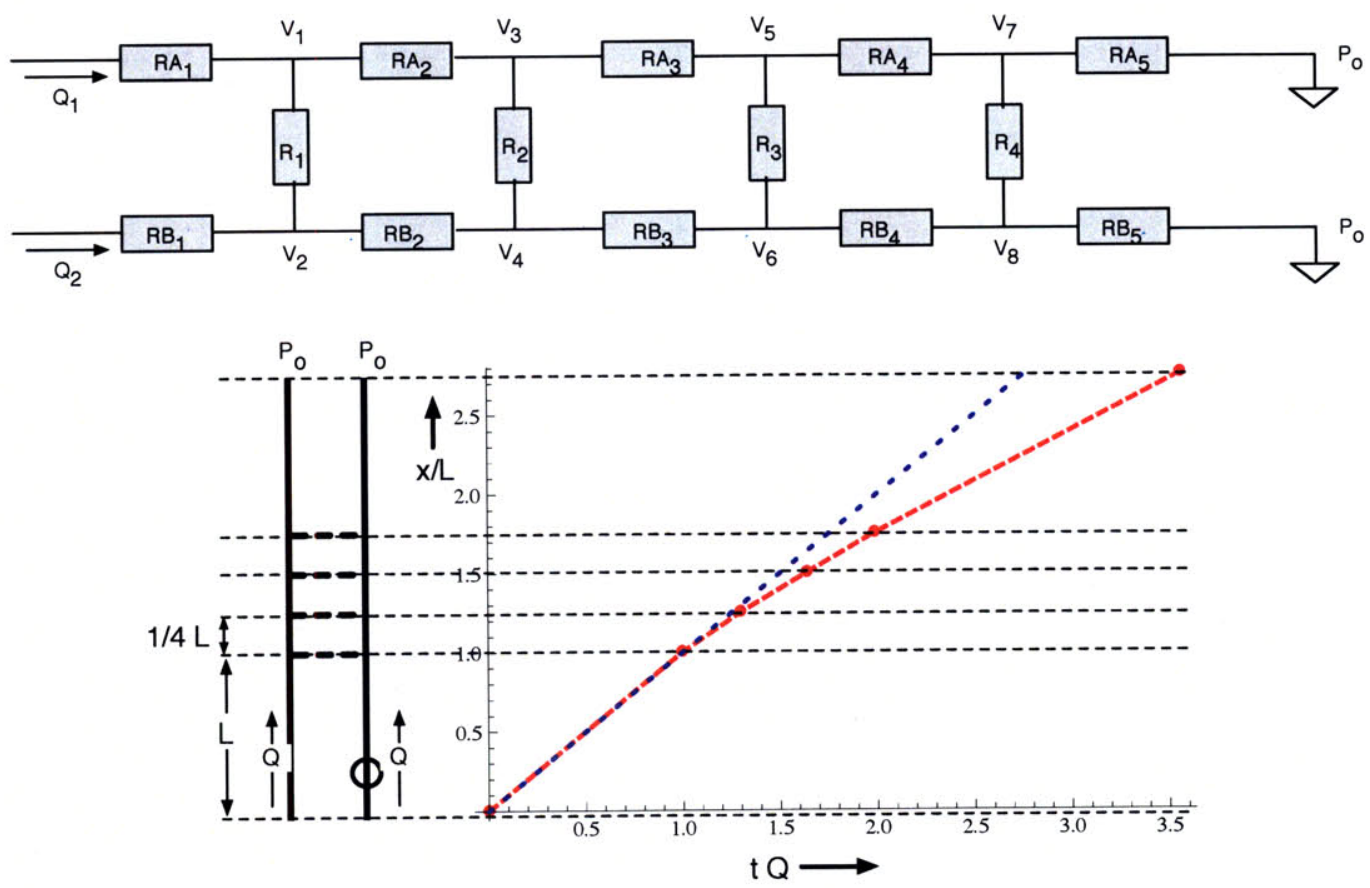

Figure 4-7: Schematic of a 4-bar ladder network for bubble synchronization, driven by constant flow $Q$ at the inlet and constant pressure $P_{o}$ at the outlet. The plot shows a time vs. distance plot for the 4-bar ladder network with $\bar{r}=8, \bar{R}_{b}=4, L_{2}=1 / 4 L_{1}$.

$$
\begin{array}{r}
I_{R A_{j}}-I_{R B_{j}}=r / R\left(i_{R_{j+1}}-i_{R_{j}}\right) \\
I_{R A_{j}}-I_{R A_{j-1}}=i_{R_{j}} \\
I_{R B_{j}}-I_{R B_{j-1}}=-i_{R_{j}} \\
I_{R A_{j}}=I_{R A_{j-1}}+2 R / r S_{j-1} \\
I_{R A_{j}}=2 \frac{R+r}{r} I_{R A_{j-1}}-I_{R A_{j-2}} \\
S_{j-1}=\sigma I_{R A_{j-1}}
\end{array}
$$

First, we consider a single bubble/droplet traversing a ladder network geometry. Analytically solving for flow-rate in channels containing the bubble for a $N=4$ channel network, 
we obtain

$$
\bar{Q}_{1}=Q_{1} / Q=\left[\frac{\bar{r}^{4}+20 \bar{r}^{3}+60 \bar{r}^{2}+56 \bar{r}+16}{\bar{r}+19 \bar{r}^{3}+48 \bar{r}^{2}+36 \bar{r}+8+\bar{r}^{3} \bar{R} b+20 \bar{r} \bar{R} b+8 \bar{R} b}\right]
$$

for bubble in first segment of the ladder, i.e. $R_{A 2}=R_{b}$. Similarly, ${ }_{2},{ }_{3}$ and ${ }_{4}$ can be evaluated. Figure 4-7 depicts time/flow-rate vs. distance plot $(t / Q$ vs $L)$ for a single bubble traversing the flow geometry for the following range of resistance values, $\bar{r}=8, \bar{R}_{b}=$ $4, L_{2}=1 / 4 L_{1}$. The velocity of the bubble is evaluated from analytical expression for $\bar{Q}_{i}$ given above.

Figure 4-10(A) shows a resistor network model of the device. Spice simulations of the geometry depict variation of relative velocity of bubble in upper channel with respect to lower channel for all possible states of the device $(\mathrm{M}, \mathrm{N})$, where $\mathrm{M}$ and $\mathrm{N}$ are the location of bubbles in the two channels at any given instant of time.

In conclusion, we have presented a novel microfluidic device for completely passive synchronization of bubbles in microfluidic networks. The scheme can also be used to synchronize droplets in microfluidic networks for coalescence and error-correction in microfluidic logic.

\subsection{Design Tools}

Integrated Computer Aided Design (CAD) tools for design, analysis and simulation of electronic circuits played a significant role in overwhelming success of the micro-electronics industry. The capability to design and analyze microelectronic systems before any effort is made on device fabrication allowed advances in circuit complexity, standardization of components and widespread training of circuit designers.

Current design and analysis tools for microfluidics are far from mature. Furthermore the problem is significantly amplified due to lack of any standardization in components. Integrated design tools for microfluidics should be able to at-least deliver on the following fronts: parametric layout capabilities with inbuilt part libraries, automated routing given device constraints, automated check for device design rules, conversion of design layout into 


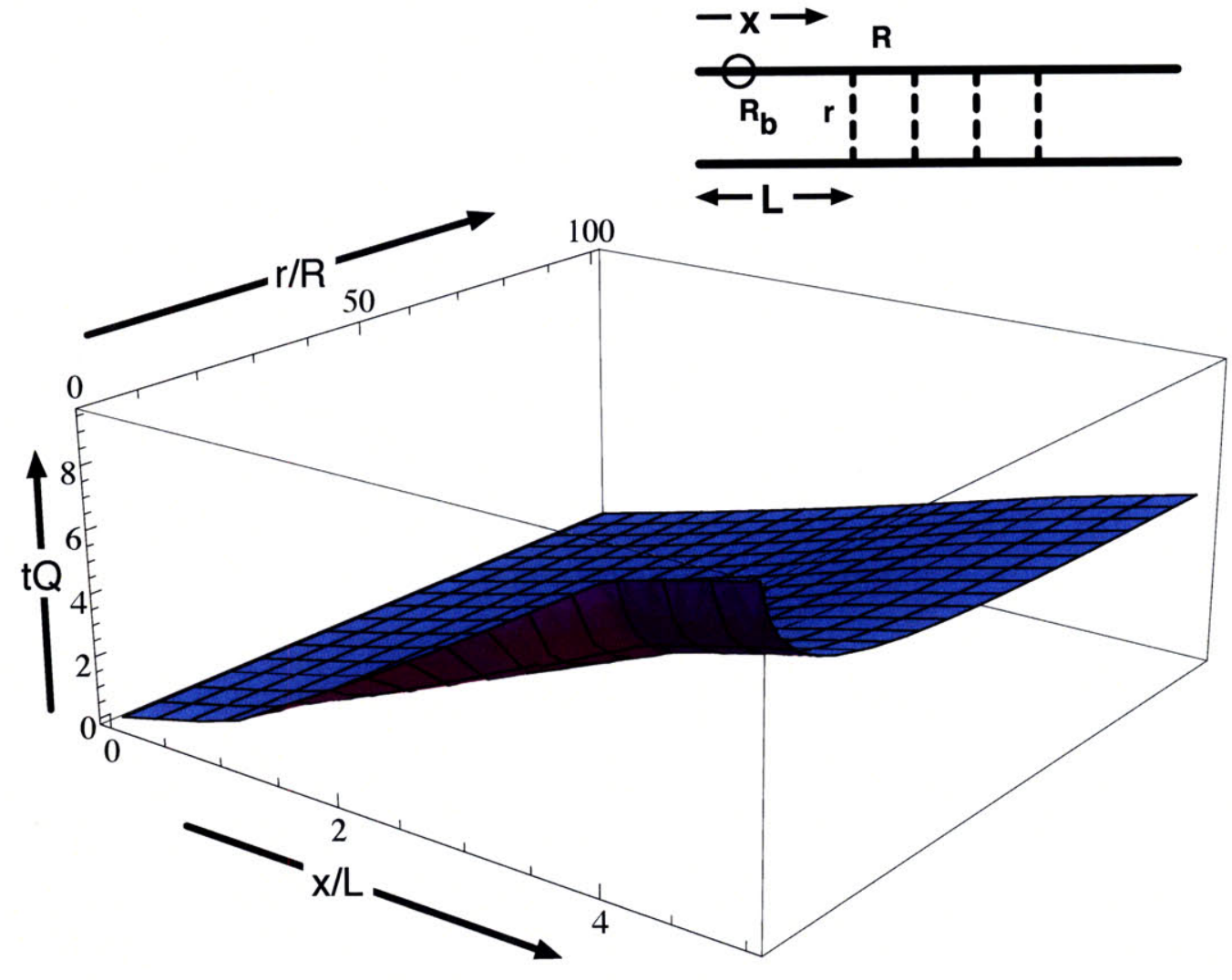

Figure 4-8: Variation of traversal velocity of a single bubbles in a ladder network characterized based on the dimensionless resistance of the bypass channels $\bar{r}=r / R$ where $R$ is the resistance of the horizontal channels, $L$ is the length of the inlet and outlet channels and $x$ is the distance travelled by the droplet from the start of the network. As can be observed, for large values of $\bar{r}>20$, the effect of the ladder network to the bubble velocity reduces drastically. For $\bar{r} \rightarrow \infty$, the bypass connections can be assumed to be non-existent. 


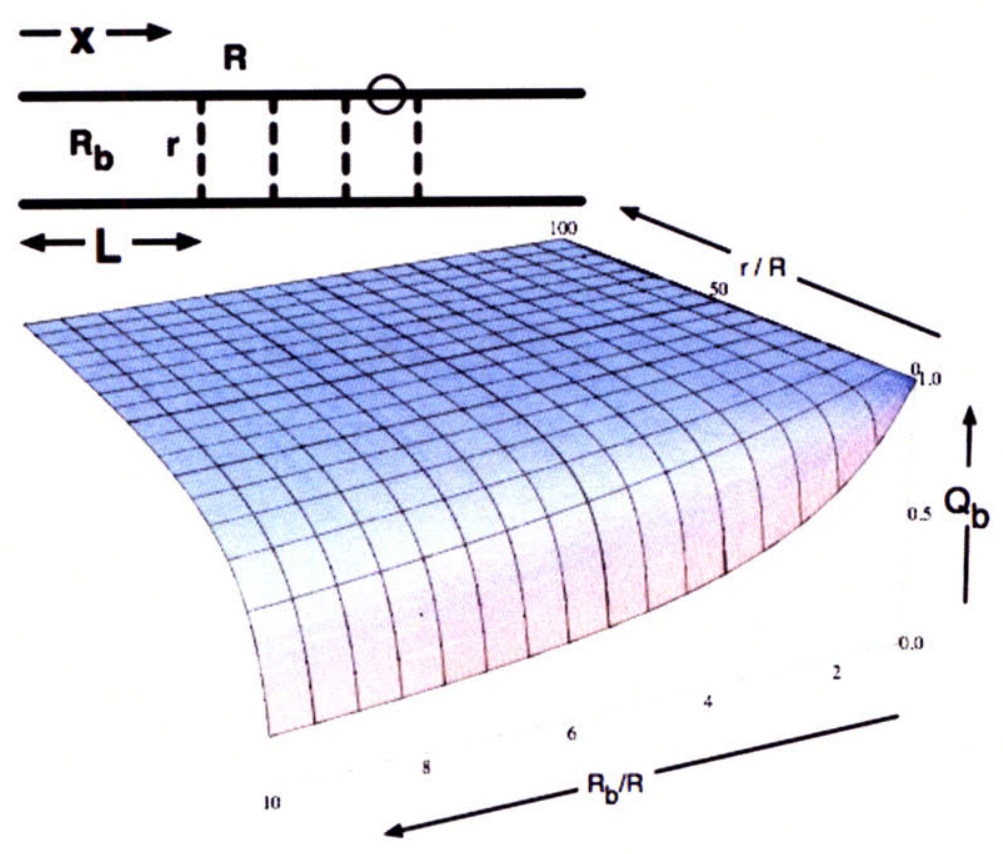

Figure 4-9: Effect of $R_{b}$ and $r / R$ on a single bubble traveling through a ladder network. As can be seen, high values of $R_{b} / R$ slows down the bubble significantly. For large values of $r / R$, the ladder network is ineffective in slowing down a bubble. 

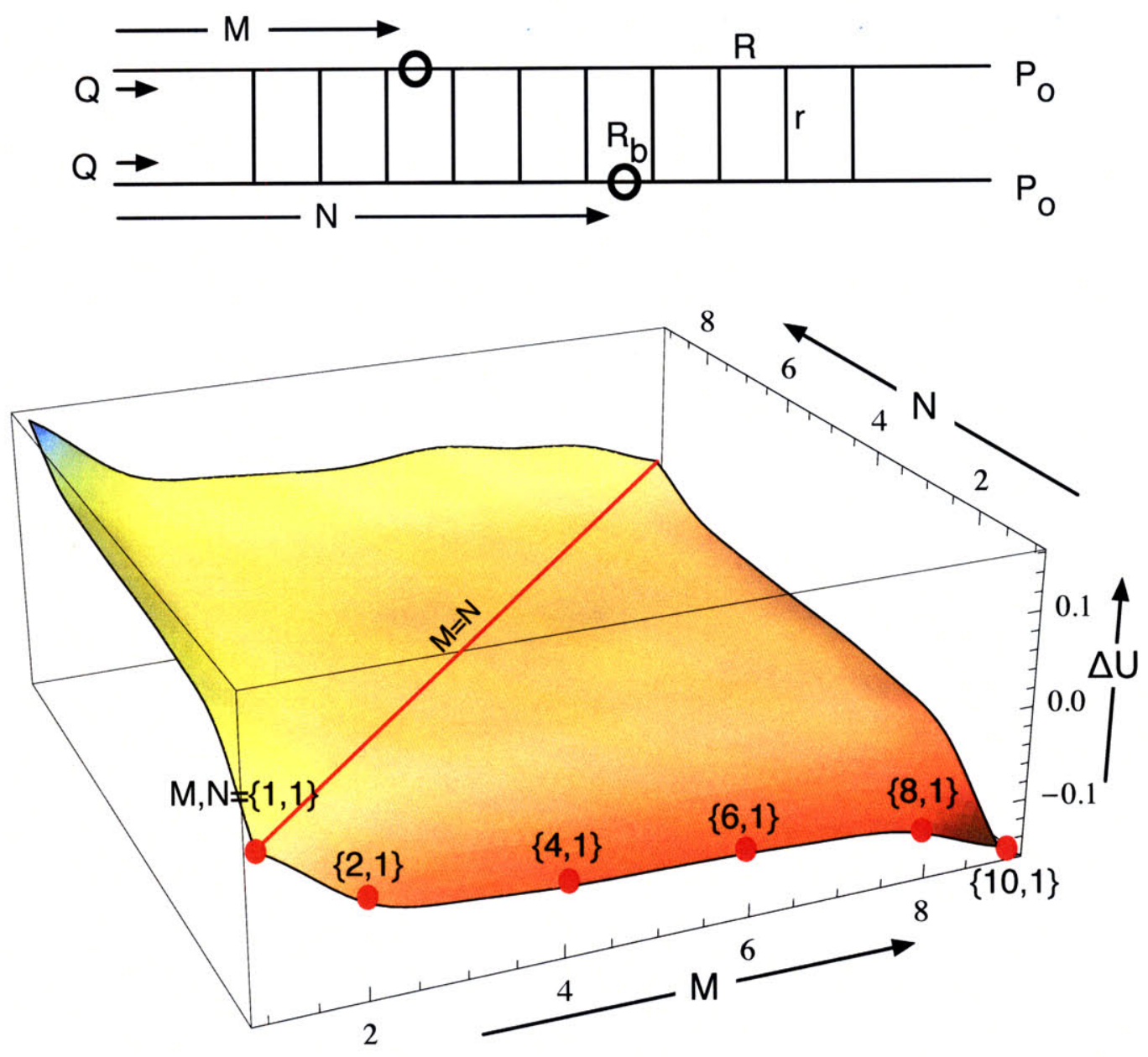

Figure 4-10: (A) Resistor network model of a synchronizer implemented with time varying non-linear resistors. The state of the system can be completely defined by three independent parameters; Q (inlet flow-rate), $\mathrm{N}$ and $\mathrm{M}$ (location of bubble in upper and lower channel). (B) Spice simulation of the resistor network model for all possible states of the LNS $\left(R_{b}=\right.$ $50 R)$. As is evident; relative velocity of bubble in upper channel (at $\mathrm{M}$ ) with respect to velocity of bubble in lower channel (at $\mathrm{N}$ ) is positive for $\mathrm{M}>\mathrm{N}$ negative for $\mathrm{M}<\mathrm{N}$; resulting in synchronization of the bubbles. For values $M=N$, the relative velocity (speed of separation, $\Delta u=0)$. 


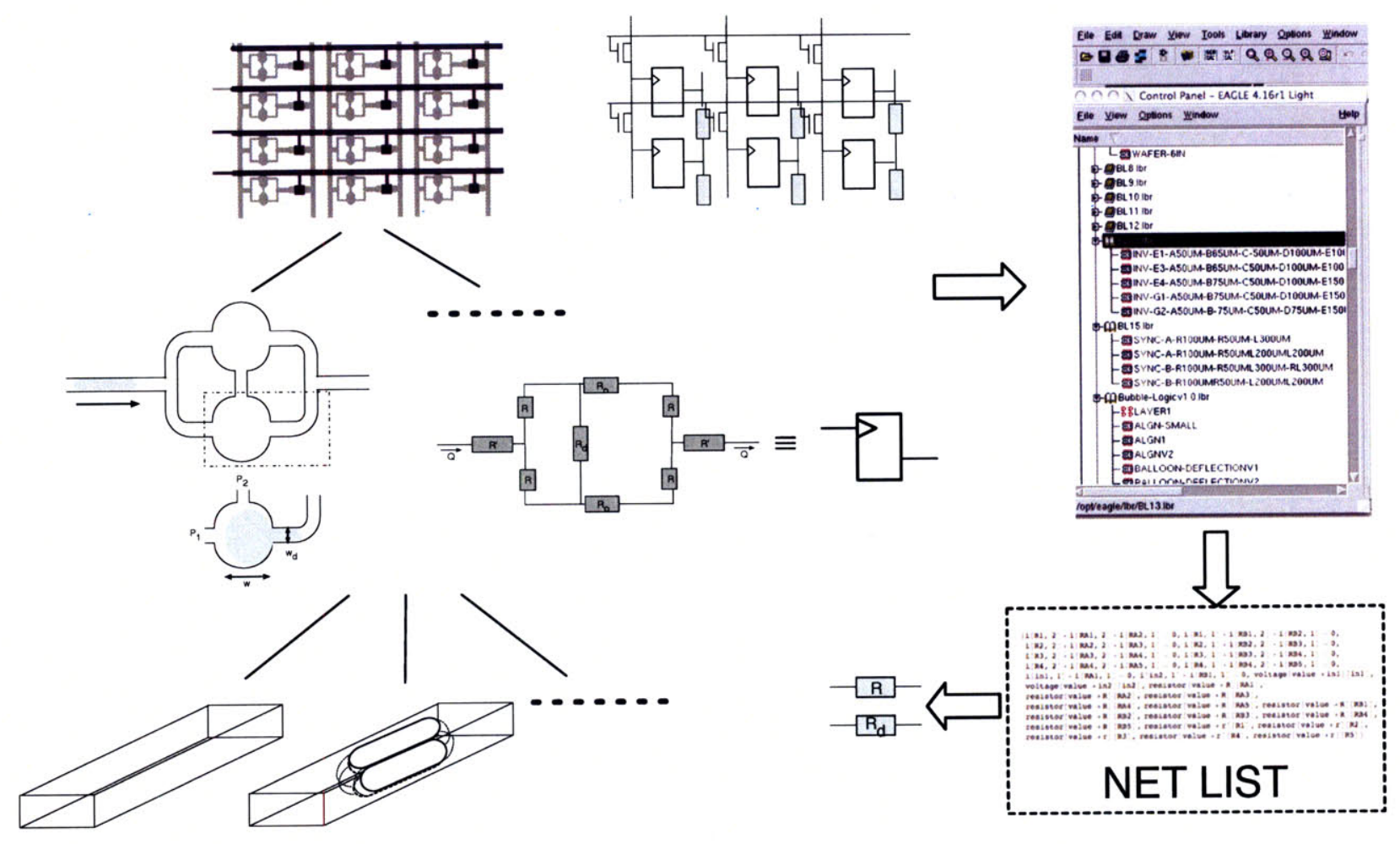

Figure 4-11: Schematic description of design and simulation tools for microfluidics similar to SPICE. A given set of fluidic channel geometries re converted to non-linear resistor network models that in turn are modeled using reduced order models for constitutive parts. A library of parts is maintained that can be called in the design tool for modular design.

circuit-level net-lists and real-time simulation of circuit characteristics. Here I list my efforts in development of an integrated environment for microfluidic circuit layout and analysis. The platform was developed on top of a open-source circuit layout tool EAGLE. Open source SPICE tools were integrated with the layout software and reduced order models for basic components were developed. A library for all parts with version control was developed which allows for easy plug-and-play design of complex circuits from basic elements. The basic components of the tool are outlined in the figure 4-11. Future work on the design tool will include integrating layout and analysis tool with automated routing scripts and further expansion of parts library. Several data sheets were developed for parts already characterized. Development of extensive data sheets for basic components will further help in standardization of parts and usage by non-experts. 


\subsection{Appendix: Symbolic evaluation of resistance networks}

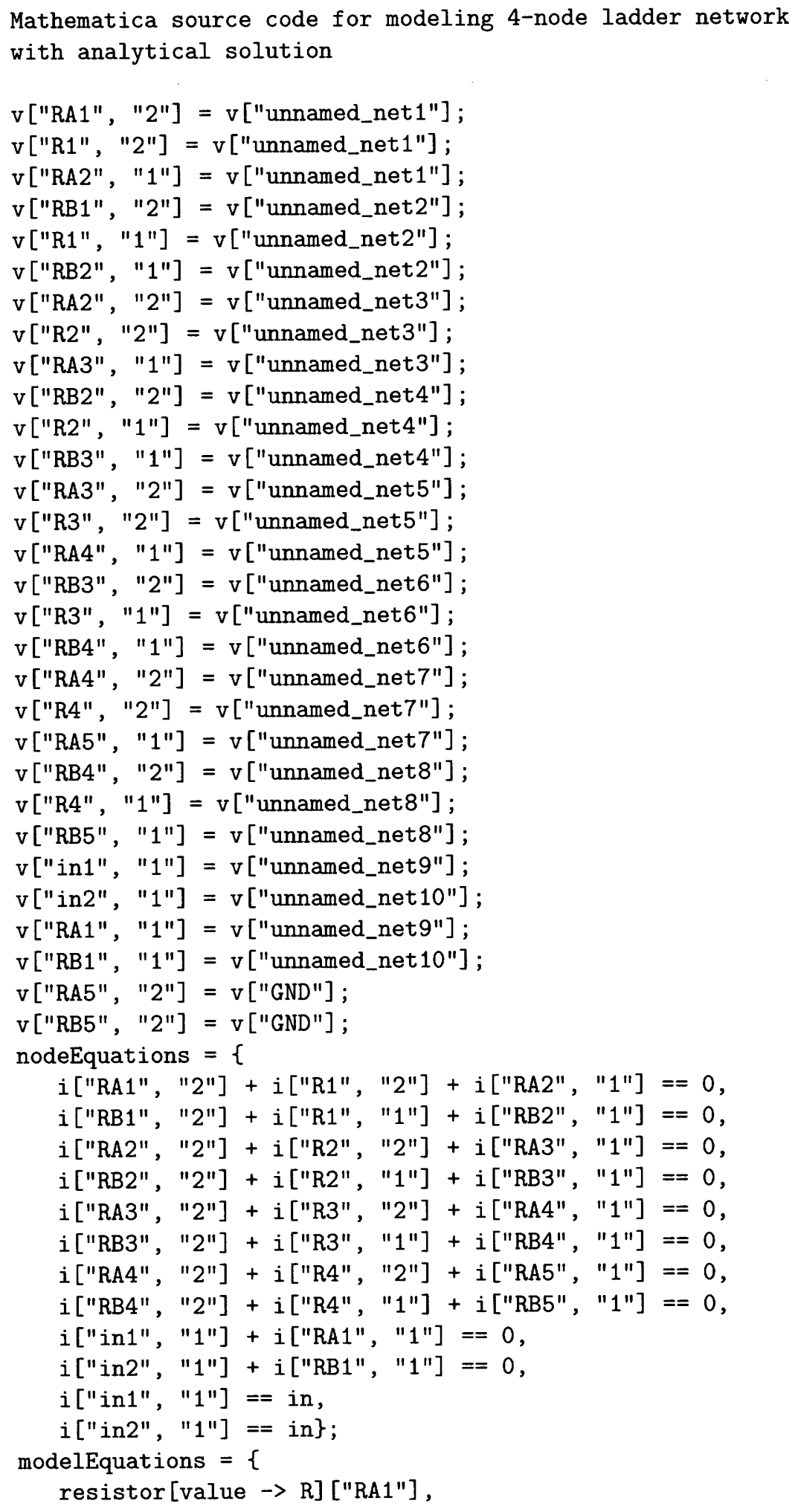




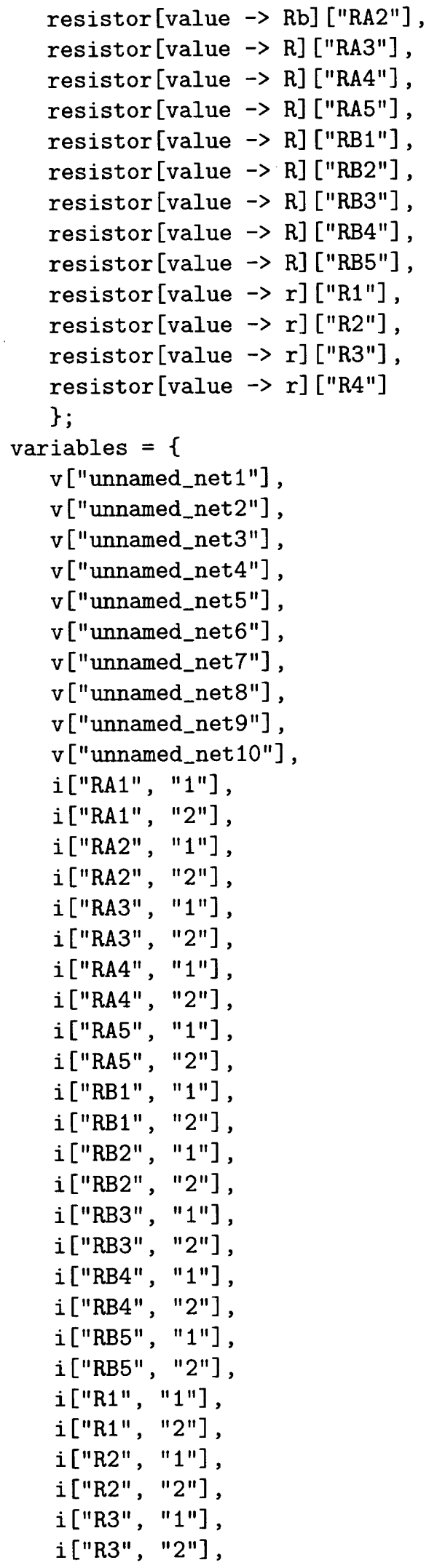


i ["R4", "1"], i["R4", "2"], i["in1", "1"], i["in2", "1"]

\}; 


\section{Bibliography}

[1] Keunho Ahn, Jeremy Agresti, Henry Chong, Manuel Marquez, and D. A. Weitz. Electrocoalescence of drops synchronized by size-dependent flow in microfluidic channels. Applied Physics Letters, 88:264105, 2006.

[2] Galder Cristobal, Jean-Philippe Benoit, Mathieu Joanicot, and Armand Ajdari. Microfluidic bypass for efficient passive regulation of droplet traffic at a junction. Applied Physics Letters, 89:034104, 2006.

[3] Mathieu Joanicot and Armand Ajdari. Droplet control for microfluidics. Science, $309(5736): 887-888,2005$.

[4] Manu Prakash and Neil Gershenfeld. Microfluidic bubble logic. Science, 315:832-835, 2007. 


\section{Chapter 5}

\section{Unidirectional superhydrophobic}

\section{surfaces}

Based on an article under review titled "Propulsion by Directional Adhesion", Bush JWM, Prakash M, submitted Sept. 2007.

\subsection{Abstract}

The water-proofing strategies of plants $[25,18]$ and insects $[5,26,30]$ have provided important guidance in the development of superhydrophobic surfaces $[12,28,11]$ that are finding myriad industrial applications owing to their ability to resist corrosion, reduce drag and self-clean $[9,19,4,16]$. The rough integument of water-walking arthropods is well known to be responsible for their water-repellency $[5,1,23,13,10]$; however, water-repellent surfaces generally experience reduced traction at an air-water interface $[19,4,16]$. A conundrum then arises as to how such creatures generate propulsive forces while retaining their waterrepellency; we here show that they do so by virtue of the distinct topology of their piliferous integument. New microscopic imaging of the interaction of arthropod cuticle and the water surface reveals that the principle propulsive forces correspond to contact forces acting through individual hairs on the driving legs. Direct force measurements reveal that the 
piliferous surface renders these adhesive forces directionally anisotropic: by virtue of the coherent tilted hair geometry, the force resisting relative motion between the fluid and cuticle is greatest for motion perpendicular to the leg. We make the key observation that the individual hairs bend in response to the capillary forces applied by the free surface, thus rendering the cuticle unidirectional: fluid glides with greatest ease towards the leg tips. These new measurements of unidirectional adhesion on the cuticle make clear its critical dynamic role, yielding new insight into the manner in which water-walking arthropods generate thrust, detach from and glide along the free surface. Furthermore, our study provides new rationale for the fundamental topological difference in the roughness on plants and water-walking arthropods, and suggests new directions for the design and fabrication of unidirectional superhydrophobic surfaces.

\subsection{Introduction}

The dynamics of water-walking insects and spiders has received considerable recent attention $[24,2,20]$, in part because it touches on the burgeoning field of dynamic water-repellency. The simplest way to rationalize the propulsion of water-walking arthropods is to note that they transfer momentum to the underlying fluid: conservation of momentum requires that they be propelled forward $[15,2]$. This physical picture is here augmented by an examination of the direct forces acting on the creature. While it is known that most water-walking arthropods rely predominantly on capillary forces for their propulsion [2], elucidating the precise nature of these forces requires consideration of the micron-scale interaction of the arthropod cuticle and air-water surface.

\subsection{Contact line adhesion}

When a water droplet wets a flat solid, the angle between the wetted solid surface and the interface, the chemical contact angle $\theta_{e}$ is prescribed by the well-known Young's equation.

The solid is defined as hydrophilic or hydrophobic according to whether $\theta_{e}$ is, respectively, 

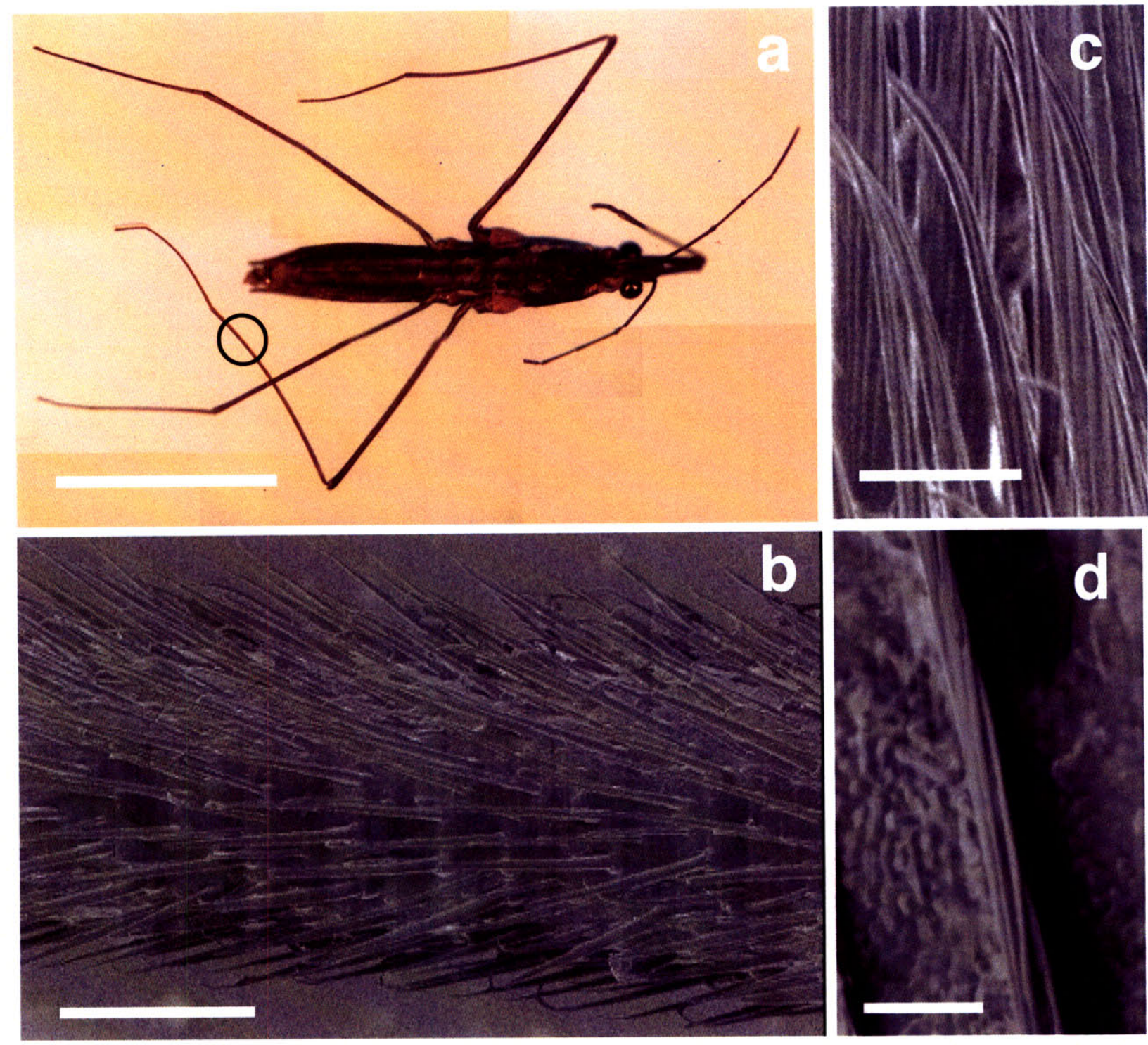

Figure 5-1: Scanning Electron Microscope (SEM) images of the rowing leg of the water strider (Gerris remigis). (a) The strider's legs are covered by an array of hairs tilted towards the leg tip. (b) The hairs have characteristic length $20 \mu \mathrm{m}$, radius $1 \mu \mathrm{m}$ and are marked by longitudinal nanogrooves (c, d) roughly 400nm wide and 200nm deep [1, 13, 9]. Scale bars: a) $1 \mathrm{~cm}$; b) $50 \mu \mathrm{m}$; c) $5 \mu \mathrm{m}$; d) $2.5 \mu \mathrm{m}$.

less than or greater than $90^{\circ}$, and superhydrophobic if $\theta_{e}>150^{\circ}$. In practice, a finite range of static contact angles may be observed [7]: static contact angles may lie anywhere in a finite range bounded above and below by the values at which contact line motion is initiated, specifically, the advancing and receding contact angles. An important consequence of this 
so-called contact angle hysteresis is a contact force that causes droplets to adhere to surfaces; for example, rain drops may stick to window panes because of the difference in the contact angles on their upper and lower edges [8]. A water-repellent surface must not only exhibit a high contact angle, but have a sufficiently low force of adhesion that impinging water droplets roll off rather than stick. Generating water-repellency generally requires the use of hydrophobic, roughened surfaces, the roughness increasing the energetic cost of wetting the solid and so discouraging fluid-solid contact [27]. When the surface is sufficiently rough, air is trapped in the inclusions, leading to a Cassie state [3] in which apparent contact angles are greatly increased, while contact angle hysteresis and the resulting contact forces are drastically diminished [6].

The leaves of the lotus flower and many other plants are water-repellent by virtue of their waxy, rough surface structure $[25,18]$ : hierarchical roughness often in the form of micronand submicron-scale bumps preserves a Cassie state. The piliferous integument of waterwalking arthropods, though topologically distinct, plays a similar role in maintaining waterrepellency and so preventing the creatures from sinking through the free surface. Critically, its surface consists of a waxy substance that increases the chemical contact angle to $105^{\circ}$ [14]. While there is a wide variation between species and body part, roughness on the legs typically takes the form of a coherent array of hairs of characteristic width $1-3 \mu \mathrm{m}$, spacing $\delta \sim 5-10 \mu \mathrm{m}$ and length $20-50 \mu \mathrm{m}$ (see $5-1$ ), tilted in the direction of the leg tips. The water strider has leg hairs tilted so as to lie roughly tangent to the leg surface (see 5-1), thereby enhancing the resistance to fluid impregnation [5]. Andersen [1] reported that the hairs of the water strider are decorated with longitudinal, fluted grooves now known to have characteristic width $400 \mathrm{~nm}$ and depth $200 \mathrm{~nm}$ [13] (5-1c,d). The importance of these grooves in increasing the apparent contact angle of the water strider to $167^{\circ}$ has recently been identified [10]; however, the dynamical significance of the linear roughness, specifically the hair and nanogroove topology, has yet to be considered. 

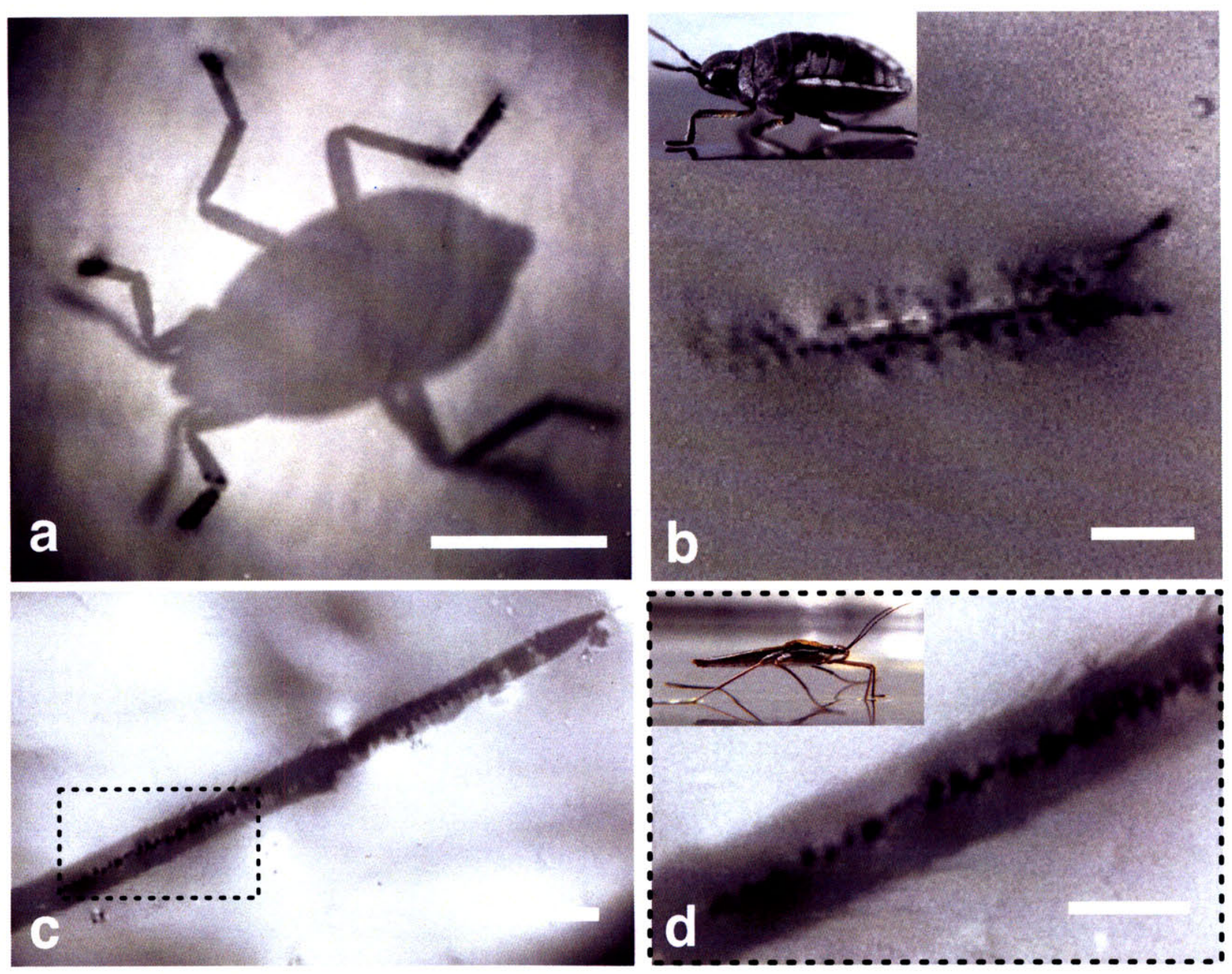

Figure 5-2: Brightfield microscope images of water-walking arthropods in a non-wetting Cassie state, captured with an inverted microscope (Zeiss LSM Pascal). a) A live Microvelia standing on the water surface grooming its water-repellent legs. b) A closer look at one of the supporting feet shows that contact between the cuticle and water surface arises exclusively through pin-holes (dark spots) associated with individual hairs. c) Contact line of the leg of a live water strider (Gerris remigis) standing on the water surface. d) Magnification of the marked region reveals that contact between insect and fluid arises exclusively through a single row of hairs. Scale bars: a) $1 \mathrm{~mm}$; b) $100 \mu \mathrm{m}$; c) $250 \mu \mathrm{m}$; d) $100 \mu \mathrm{m}$.

\subsection{Experiments}

In order for water-walking arthropods to remain water-repellent, a Cassie state must be maintained in both static and dynamic settings. 5-2 provides the first photographic evidence that the cuticle of both Microvelia and the water strider are in Cassie states as they move 
freely on the water surface: the contact between the insects and water arises exclusively through the individual hairs and an air layer is trapped between the insect leg and water surface (see Methods Summary). Maintenance of the Cassie state on a solid with roughness of characteristic scale $\delta$ requires that the fluid pressure not exceed the curvature pressures $\sigma / \delta$ generated as the fluid tries to impregnate the rough solid [22]. Balancing this curvature pressure with the dynamic pressure, $\rho U^{2}$, generated by the driving stroke or raindrop impact yields a wetting speed $U_{w} \sim(\sigma / \rho \delta)^{1 / 2} \sim 2 \mathrm{~m} / \mathrm{s}$ for the geometry of strider cuticle evident in $5-1$, where $\delta \sim 10 \mu \mathrm{m}$ is the characteristic spacing between hairs. A series of experiments in which droplets of characteristic diameter $0.5 \mathrm{~mm}$ were propelled towards the leg of a water strider confirmed a wetting speed of approximately $1.5 \mathrm{~m} / \mathrm{s}$, but also indicate a strong dependence on the location and geometry of impact, and the age and cleanliness of the sample. We note that this wetting speed exceeds the peak leg speed of the adult water strider (approximately $70 \mathrm{~cm} / \mathrm{s}$ ), but may be exceeded by the speed of incident raindrops, which thus pose the greatest danger to water-walking arthropods in terms of wetting.

Water-walking arthropods must not only maintain their water-repellency, but propel themselves through their interaction with the interface. These two requirement are at odds owing to the drag-reducing characteristics of superhydrophobic surfaces: when a rough surface in a Cassie state advances through a fluid, the opposing viscous stresses may be substantially reduced owing to the air layer adjoining the solid [16]. Nevertheless, these dual requirements may be achieved with anisotropic roughness [19, 4, 16]. Choi et al. [4] examined the fluid flow past a surface in a Cassie state sustained by microchannels of characteristic width and depth $200 \mathrm{~nm}$. Drag was found to be reduced and enhanced relative to that on a smooth surface for flow directions respectively parallel and perpendicular to the grating. Yoshimitsu et al. [29] examined the force of adhesion that acts on water droplets suspended in a Cassie state on tilted surfaces decorated with grooves of characteristic width $50 \mu \mathrm{m}$. Drops rolled with greatest ease in the direction of the grooves and with greatest difficulty perpendicular to them. We here demonstrate that the tilted, grooved topology of the hairs of waterwalking arthropods similarly serves to reduce the drag on legs gliding along their length, while increasing the resistance to leg motion perpendicular to the direction of motion. 

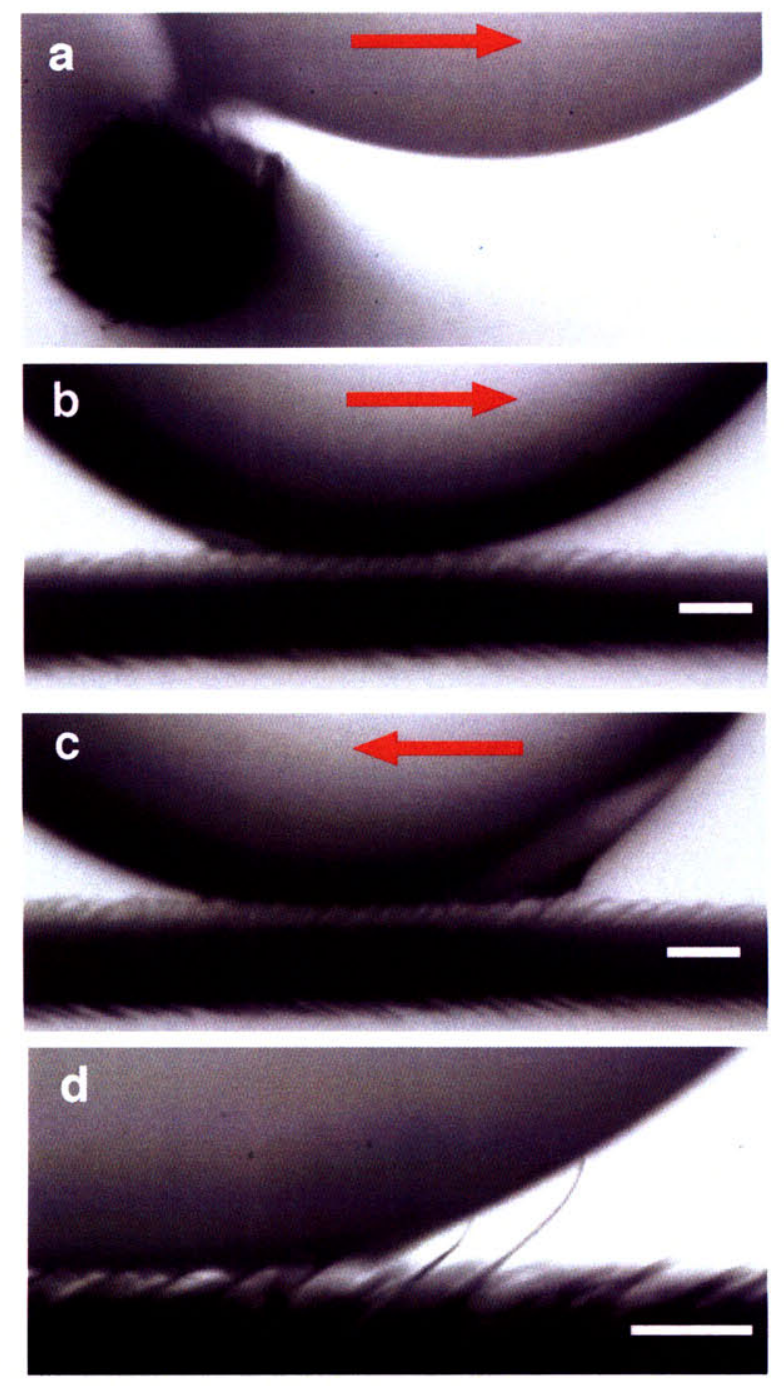

Figure 5-3: The interaction of insect cuticle with a translating water droplet. a) For drop motion perpendicular to the leg, severe drop deformations indicate large adhesive forces. $\mathbf{b}$ ) The drop moves with relative ease towards the leg tip, with little distortion of either hair or interface. c) For motion against the grain of the tilted hairs, the interface is snagged on the elastic hairs and drawn out into a thin sheet evident as a white triangle. d) Individual hairs are deflected by capillary forces as the drop advances against the grain. Scale bars a) $50 \mu \mathrm{m}$; b) $100 \mu \mathrm{m}$; c) $100 \mu \mathrm{m}$; d) $10 \mu \mathrm{m}$.

\subsubsection{Force measurement}

Unidirectional adhesion on the driving leg of the water strider can be inferred by examining the leg's interaction with a water droplet under a microscope (5-3). As the droplet was 
brushed against the leg, a Cassie state was maintained. In interpreting 5-3, it is important to note that droplet deformation reflects an increase in surface energy; therefore, motions generating the largest deformations are the most strongly resisted. For example, the severe drop distortions generated by motion perpendicular to the leg (5-3a) indicate large adhesive forces. Consistent with Yoshimitsu et al.'s [29] observations of directional adhesion on a nanograting, the surface distortions indicate that the drop translates with greatest ease parallel rather than perpendicular to the leg. Moreover, the drop advances more easily towards the leg tip than in the opposite direction owing to the flexibility of the tilted hairs. When the drop moves towards the leg tip (with the grain), the hairs and interface are largely undeformed by their relative motion (5-3b). Conversely, when it moves against the grain, the droplet interface is strongly distorted (5-3c) and advances by a series of discrete depinning events from sharply bent hairs (5-3d). Following depinning, the interface retracts and the hairs snap back into position.

The unidirectionality of the cuticle was quantified by direct measurement of the force exerted by an advancing droplet using a micro-force measurement apparatus[17] that yields direct force measurements accurate to 0.05 dynes (5-4a-c). Precise control of the distance between droplet and leg allowed us to examine the dependence of the contact force on penetration depth of the hair tips (5-4c). Contact forces increased monotonically with penetration depth, indicating that water-walking arthropods may increase their propulsive force by pushing down on the interface during their driving stroke. 5-4b illustrates the force experienced by the driving leg as it is brushes past the droplet in directions parallel and perpendicular to its length at a uniform speed of $1 \mathrm{~mm} / \mathrm{s}$ and penetration depth $h=10 \mu \mathrm{m}$. The discrete jumps in the contact forces apparent for motion parallel to the leg are associated with the depinning of the droplet surface from individual hairs. The anisotropy of the integument was always apparent, regardless of penetration depth, with the adhesive forces for motion with the grain, against the grain and perpendicular to the leg typically varying in the proportions 1 to 2 to 4 . The maximum contact force is that perpendicular to the driving leg, whose magnitude is bounded by the product of the surface tension and the perimeter of the wetted leg region. Integrating around the contact area of the water strider's driving legs suggests a total propulsive force of order 140 dynes that is roughly consistent with 
inferences based on rough scaling arguments $[15,2]$ and direct in vivo measurements [20]. For comparison, our measurements indicate that the force required to extract a leg along its length is less than 1.0 dyne.

\subsection{Methods}

The integument of the tarsal segment of the rowing leg of a freshly captured adult water strider Gerris remigis was examined using a scanning electron microscope at various magnifications (5-1). Direct evidence of the integument preserving a Cassie state was achieved via inverted microscopy of Microvelia and adult water striders moving freely on the water surface (5-2). Brightfield images were obtained with an LSM Pascal Zeiss microscope with an inverted stage. Images illustrating the interaction of the integument with a suspended water droplet (5-3) were recorded using a high-speed video camera while the drop was translated manually. A Cassie state was preserved at all times. Direct measurements of the adhesive force between droplet and driving leg were obtained with a custom-built microforce sensor using a d'Arsonval Galvanometer. Drops were directed at a uniform speed $U$ between 0.2 and $1 \mathrm{~mm} / \mathrm{s}$ with a motorized actuator coupled to a motion controller. Leg samples were mounted on the galvanometer needle, along with a platinum-coated mirror. Deflections of a laser beam shone on the mirror were measured by a quad-cell photodetector. A feedback circuit kept the needle locked in place by providing an equivalent torque via the Galvanometer coil. The measured current through the coil is proportional to the force applied on the leg sample. The penetration depth $h$ of the hair tips into the hanging droplet was controlled via a fine-motion motorized micrometer with positioning accuracy of $100 \mathrm{~nm}$. Twenty recordings were made on each of three freshly prepared tarsal leg segments. Three characteristic curves from such recordings obtained at $U=1 \mathrm{~mm} / \mathrm{s}, h=5 \mu \mathrm{m}$ are presented in 5-4b. The dependence of force on $h$ is shown in 5-4c. Additional experimental details are provided in the online Methods section.

All insects were captured at Fresh Pond in Cambridge, MA. The insects were maintained in laboratory aquariums during the course of the experiments, and sustained by a regular 


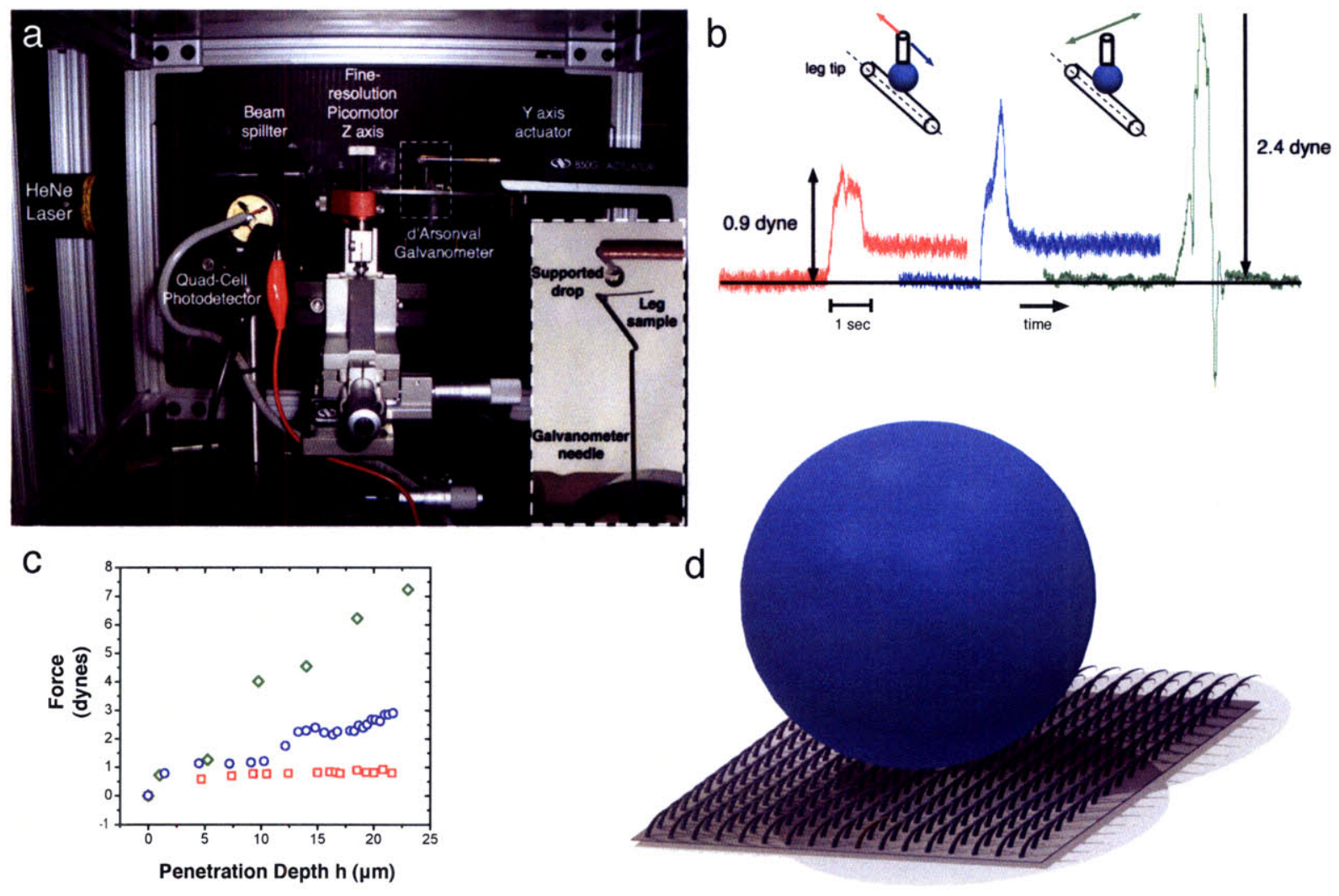

Figure 5-4: Direct measurements of the adhesive forces generated by a water droplet interacting with the driving leg of a water strider. a) The experimental apparatus. b) The evolution of the force per unit length on the leg as the water droplet is translated past it in directions perpendicular to the leg (green), and parallel to the leg, both against (blue) and with (red) the grain. The mean penetration depth of the hair tips $h=10 \mathrm{~m}$. The peaks correspond to dynamic force interactions. c) The dependence of the three contact-force components on $h$. d) The 'Bug Rug': an idealized unidirectional superhydrophobic surface based on the form of water-walking arthropod cuticle. 
diet of terrestrial insects.

\subsubsection{SEM imaging}

The integument of several water-walking arthropods was examined using a scanning electron microscope (XL30 ESEM) at various magnifications. 5-1 illustrates an adult water strider Gerris remigis, and close-ups of its driving leg. No treatment was applied prior to SEM imaging.

\subsubsection{Inverted microscopy}

Live Microvelia and adult water striders were imaged using an inverted bright field microscope (LSM Pascal 5, Zeiss Confocal microscope) with an inverted stage. Insects moved on the water surface contained within a sample vial (cross sectional area $5 \mathrm{~cm}$ by $5 \mathrm{~cm}$ ) with its bottom surface replaced by a transparent cover slip of thickness $1 \mathrm{~mm}$. Images were captured when the insects entered the microscope's field of view. 5-2a shows a live Microvelia in the process of grooming. The images were captured at various magnifications using an AxioCam HRM monochromatic camera and Axiovision software from Zeiss. The field of view was sufficiently distant from the side walls to avoid the menisci and so ensure a flat focal plane.

\subsubsection{Preparation of tarsal leg samples}

Eight tarsal leg segments, $0.5 \mathrm{~cm}$ in length, were cut from the rowing (middle) legs of four different adult water striders and mounted on a $100 \mu \mathrm{m}$ steel pin using a quick curing two part epoxy (Hardman). Care was taken not to contaminate the samples during mounting. The samples were stored in a clean sealed container at room temperature for the duration of the experiments and used the same day that they were cut and mounted. Contamination of the leg surface has an adverse effect on the water-repellency of the samples; thus, extended 
storage of the samples was avoided. If wetted during the course of an experiment, the integument would dry at room temperature via evaporation in less than 5 minutes.

\subsubsection{Contact force measurements}

Six tarsal segments were used in our measurements of contact forces. Direct measurements of forces generated by brushing water droplets with insect integument were made using a d'Arsonval Galvanometer (Simpson Electric Co. SK525-457-4), based on the micro-force measurement apparatus of Livesay \& Belser[17] that yielded a resolution of 0.05 dynes. Tarsal leg segments were mounted on the Galvanometer needle, along with a $2 \mathrm{~mm}$ by 2mm Platinum-coated silicon mirror. The deflection of an HeNe laser (Melles-Griot) beam from the mirror was recorded by a quad cell photodetector (SPOT-9DMI, UDT Inc.) after passing through a beam splitter. A low-noise voltage pre-amplifier (SR 560, Gain 200) was used to amplify the signal and control the input current in the Galvanometer coil. The torque supplied by the Galvanometer coil exactly balances the dynamic torque applied by the water droplet on the leg segment. The current in the Galvanometer coil, recorded via an $\mathrm{A} / \mathrm{D}$ convertor ( $1 \mathrm{kHz}$ sampling rate, LabView software) and analyzed using Matlab software, is proportional to and so yields the force applied on the leg sample. The entire instrument is isolated from vibrations and enclosed in a sealed plexiglass container to avoid drafts. The distance between the leg segment and the suspended droplet is precisely controlled with a piezo-based motorized micrometer (Picomotor 8301, Newfocus) with a positioning accuracy of $100 \mathrm{~nm}$. Varying the distance of the droplet from the leg segment allowed us to measure the dependence of the contact forces on the penetration depth of the hair tips. 5-4c illustrates measurements from multiple runs on the same leg segment.

Water droplets $2 \mathrm{~mm}$ in diameter were supported by a 23 gauge blunt needle mounted on a computer controlled linear actuator (Newport 850G) and translated above the leg segment at a fixed speed between $0.2 \mathrm{~mm} / \mathrm{sec}$ and $1 \mathrm{~mm} / \mathrm{sec}$ in directions parallel and perpendicular to the leg segment. Calibration tests were conducted using a pre-calibrated force transducer GSO-10 (Transducer Techniques Inc.) with a force resolution of 5 dynes. Fifteen data sets were recorded in total from three tarsal leg segments from three different 
adult water striders. A high-speed video camera (Phantom V5.0, $1000 \mathrm{fps}$ ) recorded the translation sequence. Care was taken to ensure that the leg remained in a Cassie state at all times, as was evident by virtue of the air film between the leg and droplet. Representative results are presented in 5-4.

\subsubsection{Droplet impact experiments}

Tarsal leg segments were mounted horizontally on a Newport micrometer stage. Spherical water droplets ranging in diameter from 50 to $200 \mu \mathrm{m}$ were generated from the breakup of a water jet expelled from a tapered capillary. 20 experiments involving over 200 impacts were performed on three different adult water strider legs. Wetting speeds ranging from 100-300 $\mathrm{cm} / \mathrm{s}$ were observed to depend on the droplet size, precise impact geometry, location on the leg, and age and cleanliness of the sample.

\subsubsection{High speed imaging of the rowing stroke}

The rowing stroke of an adult water strider was recorded using an inverted microscope (Zeiss STEMI 2000) coupled to a high-speed video camera (Phantom V5.0, $3000 \mathrm{fps}$ ). Following the power stroke, the driving leg is extracted along its length in a peeling motion. The freely moving strider was confined to a small water surface $(5 \mathrm{~cm}$ by $5 \mathrm{~cm})$ in order to increase the chances of capturing its motion in the field of view.

\subsection{Conclusion}

Our observations of the unidirectional adhesive properties of arthropod integument yield novel insight into the geometry of the driving stroke of water-walking arthropods. For example, the fisher spider [24] and water strider [1, 15] row, striking or brushing the surface with their driving legs tangent to the surface and perpendicular to the direction of motion: the resulting contact forces are thus maximized by both the orientation and grooved topology 
of the hairs. In order to extract their legs from the free surface following the driving stroke, they withdraw them along their length through a peeling motion. The grooves on the hairs thus serve both to increase thrust during the driving stroke, and to decrease the force of extraction. Moreover, by aligning their rear legs with the direction of motion and lifting their front pair of legs off the free surface during the driving stroke, they minimize their resistance to forward motion. Our study sets the stage for a more extensive examination of the correlation between the cuticle topology and propulsive efficiency of water-walking arthropods.

Our observations provide the first direct evidence of the flexibility and dynamic role of insect cuticle. The contact forces generated by the driving stroke, as enhanced by the anisotropy of the insect cuticle in conjunction with the stroke geometry, provide the basis for the propulsion of water-walking arthropods. The emergent physical picture of the most specialized water-walking arthropods is thus only partially consistent with the nickname of their most common representative, the water strider or 'pond skater'. Their tarsi remain in the Cassie state and, by virtue of the tilted hair geometry, act as the blades on a skate: when the legs strike the surface perpendicular to their direction of motion, they optimally generate thrust through contact forces. When the legs are aligned with the direction of motion, they experience reduced drag and glide readily along the free surface. However, the unidirectional adhesion makes the legs more like traditional cross-country skis than skates, with the flexible, tilted hairs serving as a natural herringbone. The grooved hairs thus serve not only to increase the static contact angle on arthropod cuticle $[13,10]$ but play a key propulsive role in maximizing thrust during the driving stroke, minimizing the force of extraction, and minimizing drag during the gliding phase.

Despite a number of interesting exceptions (e.g. the rice plant [9]), plant leaves are generally characterized by isotropic roughness $[25,18]$. Conversely, the leg cuticle of most waterwalking insects is distinctly anisotropic, characterized by grooved hairs pointing towards the leg tips. This distinct form of roughness enables the integument to play the dual role of retaining water-repellency while enhancing propulsive forces at the interface. Finally, unidirectional superhydrophobic surfaces inspired by arthropod cuticle are currently being 
developed and are likely to find application in dynamic water-repellency and directed fluid transport in microfluidic systems[21]. For the idealized superhydrophobic surface illustrated in 5-4d, a tilted array of flexible posts ensures that drops can only advance in the direction the posts are pointing, for example, when the substrate is subjected to random oscillations. 


\section{Bibliography}

[1] N. M. Andersen. The Semiaquatic Bugs (Hemiptera, Gerromorpha): Phylogeny, Adaptations, Biogeography and Classification. Scandinavian Science Press Ltd., Klampenborg, Denmark, 1982.

[2] J W M Bush and D L Hu. Walking on water: Biolocomotion at the interface. Ann. Rev. Fluid Mech., 38:339-369, 2006.

[3] A B D Cassie and S Baxter. Wettability of porous surfaces. Trans. Faraday Soc., 40:546-551, 1944.

[4] Chang-Hwan Choi, Umberto Ulmanella, Joonwon Kim, Chih-Ming Ho, and Chang-Jin Kim. Effective slip and friction reduction in nanograted superhydrophobic microchannels. Phys. Fluids, 18:087105, 2006.

[5] D J Crisp and W H Thorpe. The water-protecting properties of insect hairs. Disc. Faraday Soc., 3:210-220, 1948.

[6] P G de Gennes, F Brochard-Wyart, and D Quéré. Capillarity and Wetting Phenomena: Drops, Bubbles, Pearls and Waves. Springer-Verlag, Berlin, 2003.

[7] R H Dettre and R E Johnson. Contact angle hysteresis II. Contact angle measurements on rough surfaces. In F M Fowkes, editor, Contact Angle, Wettability, and Adhesion, volume 43 of $A d v$. Chem. Ser., pages 136-144. American Chemical Society, Washington, DC, 1964. 
[8] E B Dussan and R T Chow. On the ability of drops or bubbles to stick to non-horizontal surfaces of solids. J. Fluid Mech., 137:1-29, 1983.

[9] X. Feng, S. Li, Y. Li, H. Li, L. Zhang, J. Zhai, Y. Song, B. Liu, L. Jiang, and D. Zhu. Super-hydrophobic surfaces: From natural to artificial. Adv. Mater., 14:1857 - 1860, 2002 .

[10] X.-Q. Feng, X. Gao, Z. Wu, L. Jiang, and Q.-S. Zheng. Superior water repellency of water strider legs with hierarchical structures: Experiments and analysis. Langmuir, $23(9): 4892-4896,2007$.

[11] Xi-Qiao Feng and Lei Jiang. Design and creation of superwetting/antiwetting surfaces. Adv. Mater., 18:3063-3078, 2006.

[12] R Furstner, W Barthlott, C Neinhuis, and P Walzel. Wetting and self-cleaning properties of artificial superhydrophobic surfaces. Langmuir, 21:956-961, 2005.

[13] X Gao and L Jiang. Water-repellent legs of water striders. Nature, 432:36, 2004.

[14] M W Holdgate. The wetting of insect cuticle by water. J. Exp. Biol., pages 591-617, 1955 .

[15] D L Hu, B Chan, and J W M Bush. The hydrodynamics of water strider locomotion. Nature, 424:663-666, 2003.

[16] P. Joseph, C. Cottin-Bizonne, J. M. Benoît, C. Ybert, C. Journet, P. Tabeling, and L. Bocquet. Slippage of water past superhydrophobic carbon nanotube forests in microchannels. Phys. Rev. Lett., 97:156104, 2006.

[17] B R Livesay and R B Belser. Instrument for measuring small frictional forces. Tribology Transactions, 12:257-265, 1969.

[18] A Otten and S Herminghaus. How plants keep dry: A physicist's point of view. Langmuir, 20:2405-2408, 2004.

[19] Jia Ou, Blair Perot, and Jonathan P. Rothstein. Laminar drag reduction in microchannels using ultrahydrophobic surfaces. Phys. Fluids, 16,:4635-4643, 2004. 
[20] P Perez-Goodwyn and Kenji Fujisaki. Sexual conflicts, loss of flight, and fitness gains in locomotion of polymorphic water striders (Gerridae). Entomol. Exper. Applic., 2007.

[21] M. Prakash and N. Gershenfeld. Microfluidic bubble logic. Science, 315:832-835, 2007.

[22] M. Reyssat, A. Pépin, F. Marty, Y. Chen, and D Quéré. Bouncing transitions in microtextured materials. Europhys. Lett., 74:306-312, 2006.

[23] G E Stratton, R B Suter, and P R Miller. Taxonomic variation among spiders in the ability to repel water: Surface adhesion and hair density. J. Arachnol., 32:11-21, 2004.

[24] R. B. Suter, R. B. Rosenberg, S. Loeb, H. Wildman, and J.H. Long. Locomotion on the water surface: Propulsive mechanisms of the fisher spider Dolomedes triton. J. Exp. Biol., 200:2523-2538, 1997.

[25] P Wagner, R Furstner, W Barthlott, and C Neinhuis. Quantitative assessment to the structural basis of water repellency in natural and technical surfaces. J. Exp. Bot., 54:1295-1303, 2003.

[26] P Wagner, C Neinhuis, and W Barthlott. Wettability and contaminability of insect wings as a function of their surface sculpture. Acta Zool., 77:213-225, 1996.

[27] R N Wenzel. Resistance of solid surfaces to wetting by water. Ind. Eng. Chem., 28:988-994, 1936.

[28] X Wu and G Shi. Production and characterization of stable superhydrophobic surfaces based on copper hydroxide nanoneedles mimicking the legs of water striders. J. Phys. Chem. B, 110:11247-11252, 2006.

[29] Zen Yoshimitsu, Akira Nakajima, Toshiya Watanabe, and Kazuhito Hashimoto. Effects of surface structure on the hydrophobicity and sliding behavior of water droplets. Langmuir, 3:5818-5822, 2002.

[30] Yongmei Zheng, Xuefeng Gao, and Lei Jiang. Directional adhesion of superhydrophobic butterfly wings. Soft Mater., 3:178-182, 2007. 


\section{Chapter 6}

\section{Capillary ratchets}

Based on a published article titled "Hydrodynamics of capillary feeding in shorebirds: Capillary ratchet" Manu Prakash, David Quere and John WM Bush, Science, Vol. 320(5878), pp. 931-934, 16 May 2008.

\subsection{Abstract}

The variability of bird beak morphology reflects diverse foraging strategies. One such feeding mechanism in shorebirds involves surface-tension-induced transport of prey in millimetric droplets: by repeatedly opening and closing its beak in a tweezering motion, the bird moves the drop from the tip of its beak to its mouth in a stepwise ratcheting fashion. We have analysed the subtle physical mechanism responsible for drop transport and demonstrated experimentally that the beak geometry and the dynamics of tweezering may be tuned to optimize transport efficiency. We also highlight the critical dependence of the capillary ratchet on the beak's wetting properties, thus making clear the vulnerability of capillary feeders to surface pollutants. 


\subsection{Introduction}

Phalaropes (Figure 6-1a) and several other shorebirds with long, thin beaks feed primarily on small crustaceans and other invertebrates[26]. By swimming in a tight circle on the water surface, they generate a vortex that draws underlying fluid and suspended prey toward the surface[21]. By pecking on the water surface at a rate of $\sim 1.5 \mathrm{~Hz}[26,24,23,15,14]$, the birds capture water droplets with characteristic scale $\sim 2 \mathrm{~mm}$ between their upper and lower mandibles. Suction cannot be used to raise the drops mouthwards owing to the open beak geometry; gravity acts to oppose the drop motion. Nevertheless, the birds succeed in raising the drops mouthward by opening and closing their beaks successively[26, 25, 15, 14, 29]. While the importance of surface tension in this process was inferred [26], the physical mechanism responsible for the droplet transport, specifically the critical role of the beak's characteristic tweezering action, has yet to be rationalized.

When a fluid drop is placed on a flat solid, the equilibrium contact angle $\theta$ between the wetted solid surface and the interface is uniquely defined by the well-known Young's equation. If $\theta \rightarrow 0$, the drop completely wets the solid, while for any finite $\theta$, the drop is said to partially wet the solid. In practice, static contact angles observed in the case of partial wetting may lie anywhere in a finite range bounded above and below by the values at which contact line motion is initiated, specifically, the advancing and receding contact angles, respectively $\theta_{a}$ and $\theta_{r}[3,8,10]$. An important consequence of this so-called contact angle hysteresis is a contact force that causes drops to adhere to surfaces; for example,

rain drops stick to window panes because of the difference in the contact angles on their upper and lower edges[11]. While contact angle hysteresis typically acts to resist the sliding of droplets on solids $[10,8]$, it may be overcome by vibration $[6,7]$. We demonstrate that, in capillary feeding, contact angle hysteresis couples to the time-dependent beak geometry corresponding to the mandibular spreading cycles[26, 25, 24, 15, 14], thereby driving drop motion via a ratcheting mechanism.

Surface tension transport relies explicitly on the bird opening and closing its beak, and so varying the beak opening angle $\alpha[26,15]$ (Fig. 6-1b). This angle has an upper bound 
since a drop pinned between two plates will break at an opening angle $\alpha_{b r e a k}$ if its height to radius ratio exceeds $2 \pi[4,19,5]$. Denoting the beak length by $L_{b}$ and the drop size by the capillary length, $l_{c} \sim(\gamma / \rho g)^{1 / 2} \sim 2 \mathrm{~mm}$, the maximum opening angle is thus of the order $\alpha_{\text {break }} \sim l_{c} / L_{b} \sim 11^{\circ}$ for capillary feeders (Fig. 6-1b; see also figure 6-5). The characteristic time to transport a drop along the beak length $L_{b} \sim 2 \mathrm{~cm}$ is $20 \mathrm{~ms}$, corresponding to mean drop speeds as high as $100 \mathrm{~cm} / \mathrm{s}[26,23]$. In Fig. 6-1b, we present the mean beak length and width of 18 shorebird species with straight bills, and demonstrate that surface tension transport is employed only by birds with the smallest beaks. Rubega[24] demonstrated that beak dimensions do not scale with body size in adult Red-necked Phalaropes, Phalaropus lobatus, suggesting the critical role of internal beak morphology in capillary feeding. Re-

cently, Estrella et. al. [15] substantially expanded the list of capillary feeders (Fig. 6-1b), underscoring the prevalence of surface tension transport. Though morphometric analysis of bird bills commonly yields insight into foraging mechanisms [20] and has lead to new understanding of feeding modes [12, 23], analytical and experimental studies of these mechanisms are exceedingly rare [17]. We here present one such study.

\subsection{Experiments}

In our experimental study, we constructed mechanical wedge-like geometries modeled after the bird beak. Mechanical beaks with a stainless steel surface were polished with a Buehler Metadi diamond slurry (average particle size $\sim 3 \mu m$ ). The surface was ultrasonically cleaned for an hour, plasma treated in oxygen for 1 minute to remove any residue, then left in air for an hour before experiments were performed. The mechanical beaks were mounted and actuated by a motorized micrometer stage such that the beak opening and closing angle could be precisely controlled by a computer. Drops of known volume (ranging from $0.5 \mu L$ to $2 \mu L$ ) were inserted via a micro-pipette at the tip of the beak. A high speed video camera (Phantom v5.0) recorded the resulting drop dynamics.

We first deposit a completely wetting fluid (silicone oil, surface tension $\gamma=0.02 \mathrm{~N} / \mathrm{m}$ ) in the form of a droplet that spans the wedge (Figure 6-2a). The drops propagate towards the 


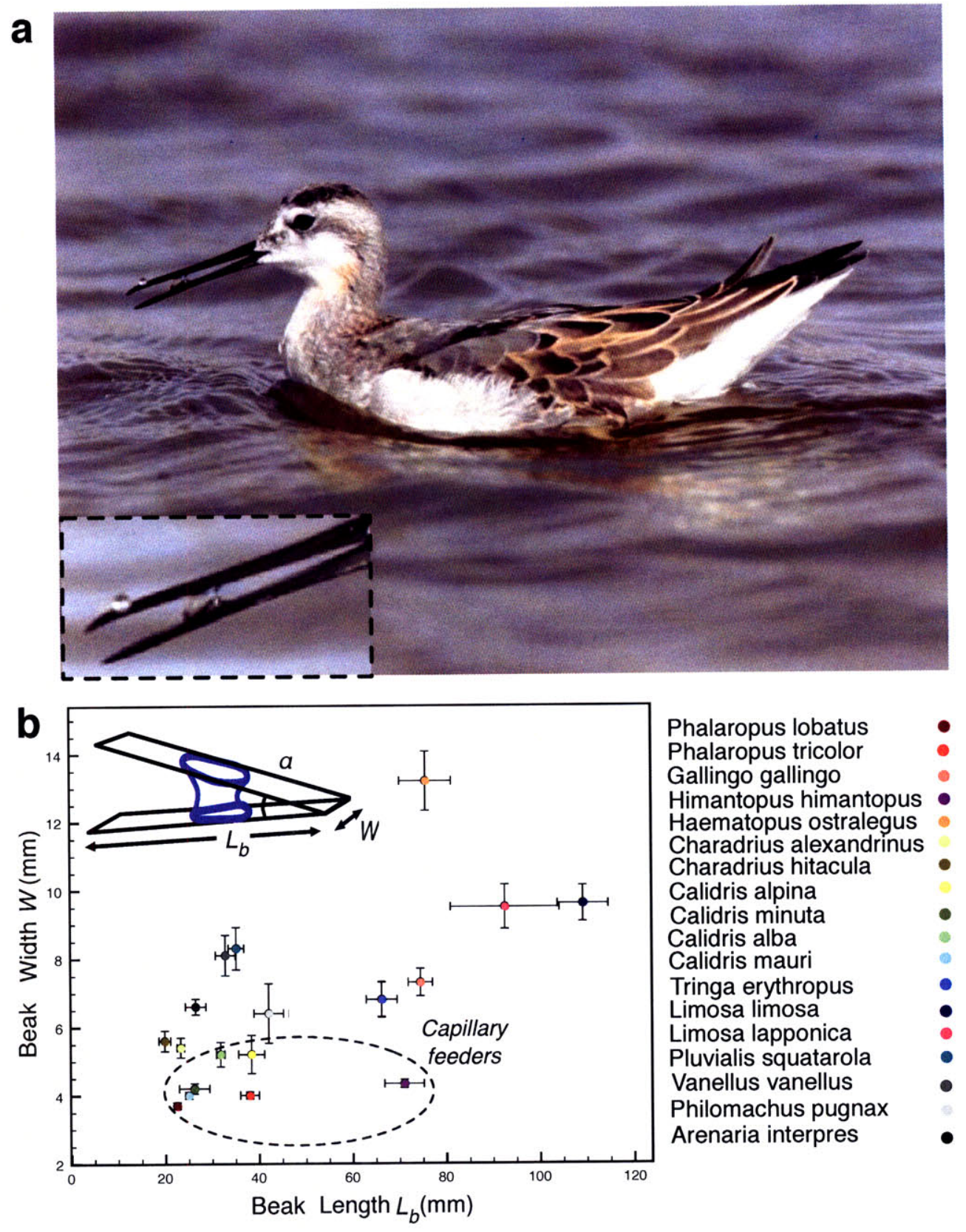

Figure 6-1: (a) A juvenile Wilson's Phalarope feeding. Note the prey suspended in the droplet trapped in its beak (inset). Photo courtesy of Robert Lewis. (b) Shorebirds employ a variety of foraging strategies [1] and so exhibit large variations in beak size and shape. Here we plot bill length and base width of common shorebirds with straight bills (data compiled from sources $[28,23,20,1])$ Scale bars represent standard deviation in the reported data. 
narrower region, advancing first at a constant speed, then accelerating as they approach the apex of the wedge (Figure 6-2b,c). The behavior in this fully wetting regime may be rationalized by simple scaling arguments (see Supplementary Online Material). The jump in pressure across a surface is proportional to the surface tension $\gamma$ and the local curvature; such curvature pressures are capable of driving fluid motion. In 1712, Hauksbee [16] reported that "oil of orange" droplets trapped between two non-parallel glass plates move spontaneously in the direction of decreasing gap thickness. Similarly, a completely wetting fluid drop confined in a conical capillary is known to self-propel towards the narrower end due to the axial force arising from differing curvature pressures across its end caps[2]. In our wedge geometry, the opening angle is denoted by $\alpha$, the width of the drop by $W$, the distance of the drop from the apex by $x$, and the length of the drop by $L$ (Figure 6-2b). The drop height is necessarily $\alpha x$. For $x>L$, the pressure difference between the two caps scales as $\gamma L / \alpha x^{2}$ and the drop volume as $\Omega \sim \alpha x L W$; hence a driving force $F \sim \gamma W L / x$ arises. For a fluid drop with dynamic viscosity $\eta$ advancing at a speed $v$, the viscous force resisting its motion is given by $F_{\eta} \sim \eta W L v /(\alpha x)$; the force balance thus yields a steady speed $v_{0} \sim \gamma \alpha / \eta$ that is independent of drop position $x$ and drop length $L$. As the drop approaches the apex, $x<L$, the pressure difference between the caps scales as $\gamma /(\alpha x)$ and the volume as $\alpha L^{2} W$. The resulting driving force now varies as $\gamma L W / x$, and the viscous resistance as $\eta W v / \alpha$. The resulting drop speed $v_{0} L / x$ diverges as the drop approaches the apex despite the progressively increasing confinement. Following a brief transient period, these two distinct regions of constant speed and acceleration are apparent in Figure 6-2c.

When water is used in place of oil, the behaviour is strikingly different: no droplet motion arises (Fig. 6-3a top row). Unlike the silicone oil, the water only partially wets the solid; consequently, the droplet motion is resisted by contact angle hysteresis. Specifically, there is an adhesive force whose magnitude scales as $\gamma W \Delta \cos \theta$, where $\Delta \cos \theta=\cos \theta_{r}-\cos \theta_{a}[8,9]$ and $W$ is the length of the advancing contact line. In our system, water droplets on stainless steel beaks have an advancing angle $\theta_{a} \sim 65^{\circ}$ and receding angle $\theta_{r} \sim 20^{\circ}$ that are roughly comparable to those of water droplets on keratin [18]. Drop motion is only possible if the capillary driving force $F \sim \gamma \cos \theta(W L / x)$ exceeds this sticking force, that is, if $\alpha>$ $\frac{\Delta(\cos \theta)}{\cos \theta} \frac{\Omega}{W L^{2}}$. This condition cannot be satisfied as both $\Delta(\cos \theta) / \cos \theta$ and $\Omega /\left(W L^{2}\right)$ are 

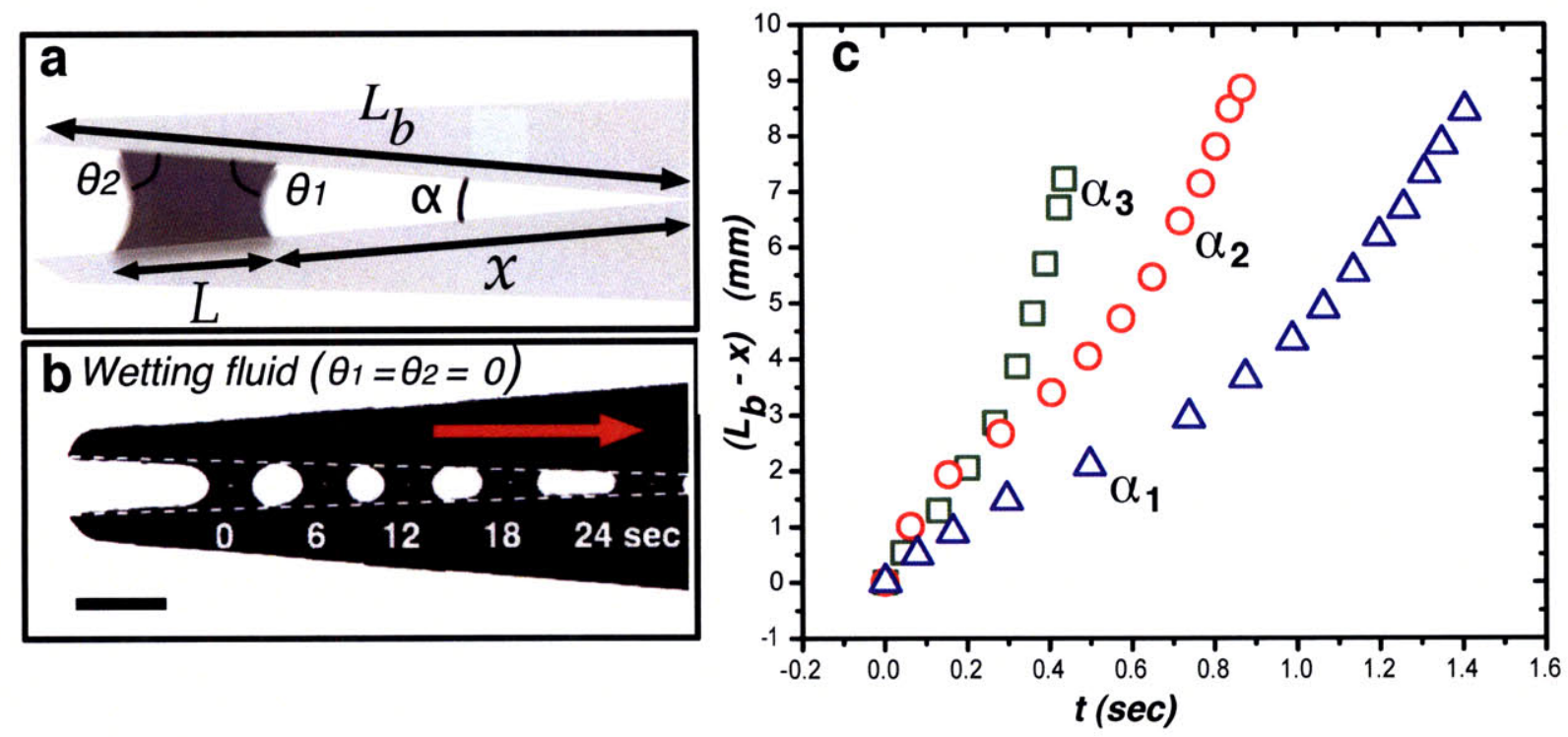

Figure 6-2: Fluid drop in a horizontal beak. (a) Schematic of a bird beak with a fluid drop trapped between upper and lower mandibles. (b) A completely wetting drop of silicone oil $\left(\theta_{1}=\theta_{2}=0\right.$, dynamic viscosity $\left.\eta=0.05 \mathrm{~kg} \mathrm{~m}^{-1} \mathrm{~s}^{-2}\right)$ self-propels towards the apex of a mechanical bird beak with a constant opening angle $\alpha=3.4^{\circ}$ and uniform width $1 \mathrm{~mm}$. Scale bar $2 \mathrm{~mm}$. (c) Plot of drop front position versus time for silicone oil $(\eta=0.01 \mathrm{~kg}$ $\left.\mathrm{m}^{-1} \mathrm{~s}^{-2}\right)$ for three opening angles $\alpha_{1}=1.9^{\circ}, \alpha_{2}=2.8^{\circ}$ and $\alpha_{3}=4.2^{\circ}$, where $\left(L_{b}-x\right)(\mathrm{mm})$ represents the distance travelled by the drop from the beak tip. 
order one quantities, while $\alpha$ must be less than $\alpha_{b r e a k} \sim 0.2$ radians for drop stability. The relatively minor influence of the fully three-dimensional geometry was examined numerically (see Supplementary Figure S1). The influence of beak taper and orientation were examined both experimentally and numerically. Realistic beak tapers[24, 15] were found to have only a weak quantitative effect on the drop propulsion, while beak orientation had a negligible effect in the ratcheting regime.

Phalaropes induce drop motion by cyclically opening and closing their beaks [26, 23, 25, 24]. We follow their lead in actuating the mechanical beak by opening and closing the wedge geometry at a constant angular velocity $\omega$, with $\alpha_{\text {close }}$ and $\alpha_{\text {open }}$ being the minimum and maximum opening angles, respectively. We record the location of both front and rear contact lines of the drop with a high speed camera mounted on a microscope (Figure 6-3a). For a given drop volume, varying $\alpha_{\text {close }}$ and $\alpha_{\text {open }}$ reveals three distinct regimes.

If $\alpha_{\text {open }}-\alpha_{\text {close }}$ is sufficiently small that the leading and trailing contact angles, respectively $\theta_{1}$ and $\theta_{2}$, satisfy $\theta_{r}<\theta_{2}<\theta_{1}<\theta_{a}$, then the drop remains pinned (Figure 6-4a). The dynamics for larger values of $\alpha_{\text {open }}-\alpha_{\text {close }}$ is best understood by considering in turn the closing and opening phases. During the closing phase, both contact lines have the tendency to progress outwards, but the leading edge (A) always does so first. During the opening phase, both contact lines tend to retreat inwards, but the trailing edge (B) does so first. The drop thus advances through a slipping ratcheting motion: in each cycle, both leading and trailing edges of the contact lines advance and retreat. Nevertheless, due to the asymmetry in the wedge geometry, net mouthward drop motion is still achieved, albeit inefficiently. When $\alpha_{\text {close }}$ and $\alpha_{\text {open }}$ are optimally tuned, the droplet advances through a pure ratcheting motion with no slippage. The two contact lines move asynchronously but progressively towards the apex: during the opening phase, the leading edge (B) remains pinned while the trailing edge retreats (A); during closing, the leading edge (B) advances while the trailing edge (A) remains pinned (Figure 6-4a). The time dependence of the contact line positions and opening angle for nearly pure capillary ratcheting is plotted in Figure 6-3(b). We note that the ratcheting motion is quasi-static, with the instantaneous position of the drop being uniquely determined by the history of the beak motion; therefore, the drop speed increases 

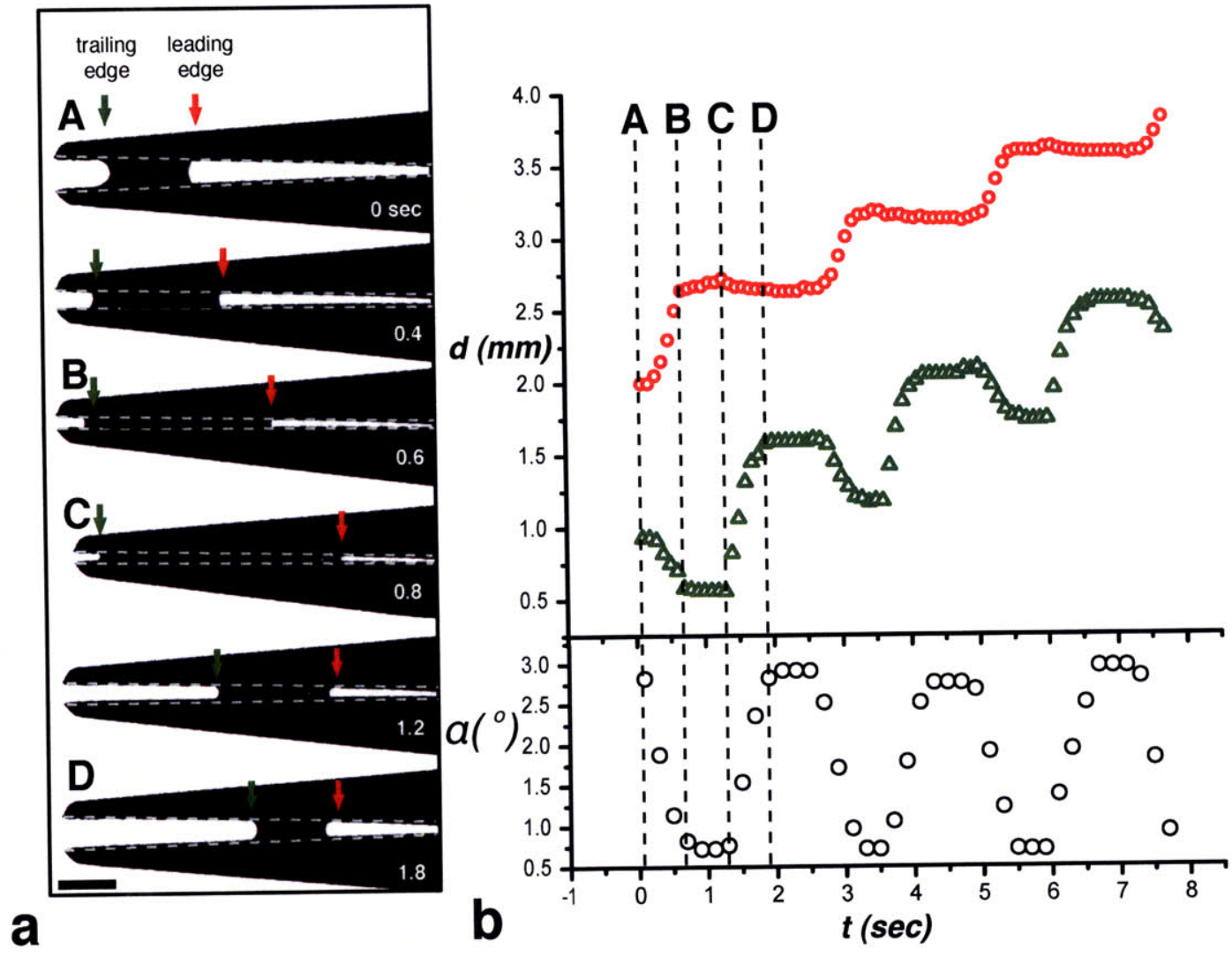

Figure 6-3: The capillary ratchet. (a) Time sequence illustrating the water droplet transport generated by an opening and closing cycle of the mechanical beak. In the closing cycle, the leading contact line proceeds towards the mouth; in the opening cycle, the trailing contact line recedes towards the mouth. The result is net drop transport towards the mouth. Scale bar, $2 \mathrm{~mm}$. (b) Plot of the associated motion of the leading (red) and trailing (green) contact lines generated by varying the opening angle $\alpha$ over three cycles. $d(\mathrm{~mm})$ represents the distance from the beak tip to the contact line. See also Supplementary Movie S2. 
linearly with the ratcheting frequency $\omega$.

Figure 6-4(b) illustrates the various regimes of droplet transport observed in our mechanical bird beak by varying the minimum and maximum opening angles, $\alpha_{\text {close }}$ and $\alpha_{\text {open }}$, respectively. In addition to regimes characterized by drop pinning and drop break-up, we report the number of cycles required for drop transport from mechanical beak tip to the apex of the wedge in the ratchet regime. For our specific combination of droplet volume $(1.5 \mu \mathrm{l})$ and mechanical beak geometry, the minimum number of cycles, three, corresponds to the most efficient capillary ratchet. It is interesting to speculate as to the degree of optimization of capillary feeding in the wild. On average, a single drop is transported from the beak tip to the buccal cavity of the Red-necked Phalarope in 2-3 mandibular spreading cycles $[26,23]$. Wilson's Phalarope are evidently less optimized for capillary feeding, and require 7-8 cycles[25]. Our observations provide a quantitative measure of efficiency of shorebird beaks in capillary feeding, and so may yield insight into their degree of adaptation. Moreover, they yield new insight into recent observations of rynchokinesis, in which capillary feeding may be enhanced by beak flexure [14].

The beaks of shorebirds may be largely vertical during capillary feeding; thus, the influence of gravity need be considered. While gravity acts to resist the climbing drop, it is overcome by contact angle hysteresis provided the pinning force, $F_{p}=\gamma W \Delta(\cos \theta)$, exceeds the drop and prey weight, $M g$. Characteristic values for the Phalarope ( $W \sim 2 \mathrm{~mm}, \Omega \sim 5-10 \mu l[26])$ indicate that $F_{p} /(M g)>1$ : contact angle hysteresis can safely support the drop's weight. In our experimental study, changing the orientation of the mechanical beaks from horizontal to vertical indeed had negligible effect on the dynamics of the water drops. Conversely, wetting silicone droplets were observed to slip downwards under the influence of gravity owing to the absence of contact line pinning. Wetting droplets would slip if the propulsive capillary force $\gamma W L / x$ is exceeded by the drop's weight. As the relative magnitudes of these forces is given by $\ell_{c}^{2} /\left(\alpha\left(L_{b}\right)^{2}\right) \sim 0.1$, with $\alpha \sim 5^{\circ}=0.1$ rad and beak length $L_{b} \sim 2 \mathrm{~cm}$, we conclude that gravity would preclude capillary feeding if the beaks were wetting. We thus see that, while the partially wetting nature of the bird beaks disables static capillary propulsion through the introduction of an adhesive force, it enables droplet transport via 


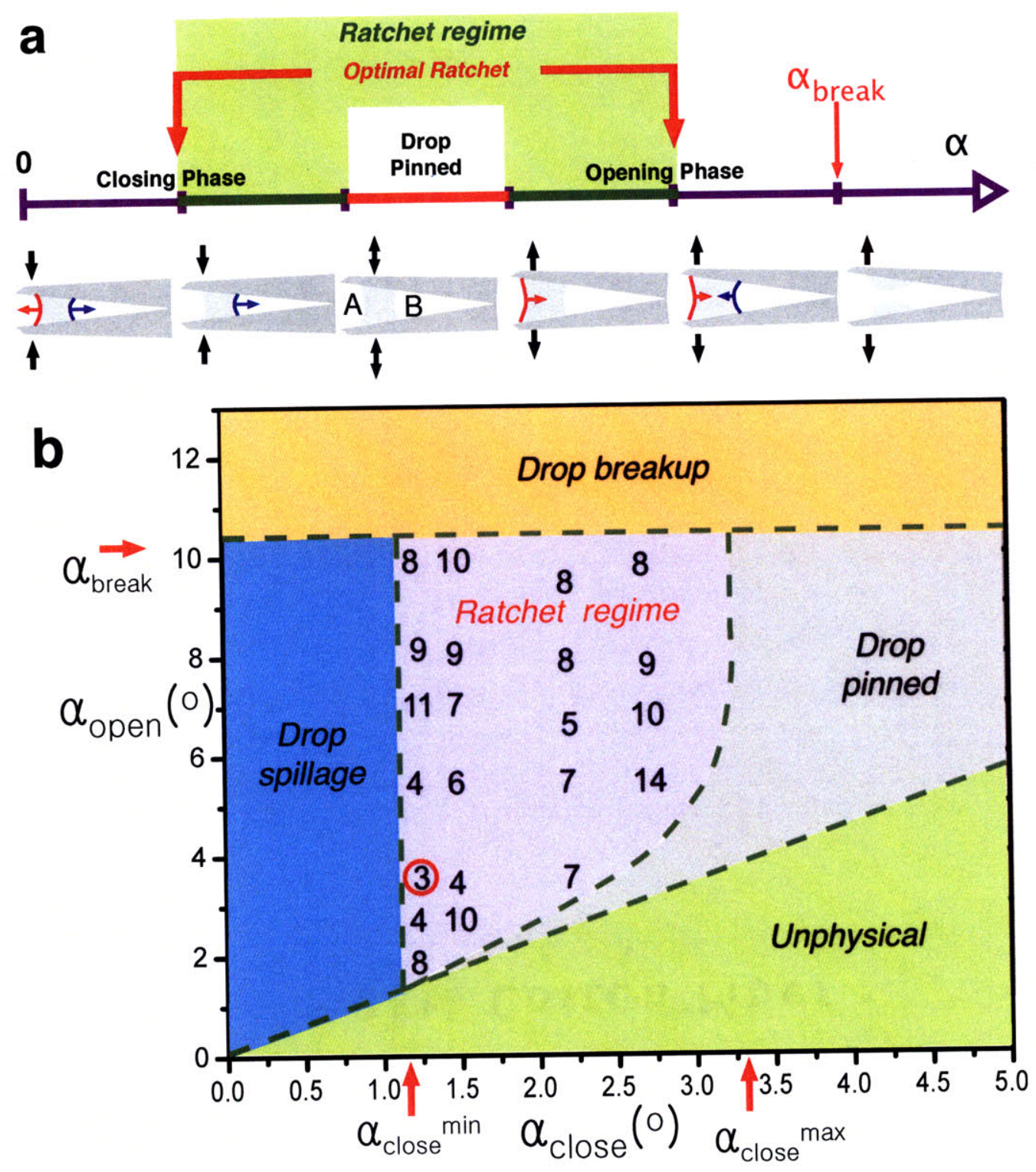

Figure 6-4: (a) A schematic illustration of droplet dynamics in an oscillating bird beak. The drop is pinned for region $\theta_{a}>\theta_{1}>\theta_{2}>\theta_{r}$, marked by the red line. As the beak is closed progressively, first the leading (A) then the leading and trailing (B) contact lines advance. As the beak is opened, first the trailing (B) then the trailing and leading (A) contact lines retreat. Ultimately, the drop breaks when $\alpha>\alpha_{\text {break }}$. The ratcheting regime is indicated in green and the optimal ratchet by the red arrows. (b) Regime diagram for droplet transport in an oscillating mechanical bird beak illustrates the dependence of the system behavior on the minimum and maximum opening angles, respectively, $\alpha_{\text {close }}$ and $\alpha_{\text {open }}>\alpha_{\text {close }}$. The drop volume was fixed at 1.5 $\mu \mathrm{l}$. For $\alpha_{\text {open }}>\alpha_{\text {break }}$, the drop breaks, while for $\alpha_{\text {close }}<\alpha_{\text {closemin }}$, the drop fills and spills from the beak. The numbers denote the number of cycles required to transport the drop from the beak tip to the mouth in the ratcheting regime. The optimal capillary ratchet transports the drop in three cycles. 
capillary ratcheting, a mechanism that naturally overcomes gravity. We thus highlight the precarious nature of capillary feeding: any surface contamination that alters the wetting properties of the beaks represents a serious threat, particularly to shorebirds such as the Red-necked Phalarope that rely exclusively on this mode of feeding[26, 25]. Given the drastic changes in wetting behaviour that accompany contamination with pollutants such as petroleum or detergents[9], our study makes clear the critical danger to this class of shorebirds posed by chemical or oil spills $[13,27]$.

\subsection{Conclusion}

Contact angle hysteresis typically resists the motion of drops on solid substrates; conversely, in capillary feeding, it couples with the time-dependent beak geometry to drive the drops. As such, surface tension transport represents a peculiarity for which contact angle hysteresis enables rather than impedes drop motion. By elucidating the dependence of the efficiency of the capillary ratchet on dynamic beak morphology, we have enabled quantitative comparative studies of capillary feeding across species. We note that the efficiency of capillary feeding may be enhanced by tuning the beak geometry, dynamics and wetting properties. Analogous mechanisms for small-scale drop transport in microfluidic systems[22] are currently being explored.

\subsection{Appendix: Surface Evolver Source Code}

// beakv1_6.fe

// Used to calculate the curve between Alpha and Volume increase

// Updated July 26, 2007

// Model for determination of drop breakup critical angle for a given volume

// Match the experiment conducted for drop breakup in a wedge

// The model uses cgs units

// Interior angle between bottom plane and surface, degrees

// Not used for the case where the drop contact line is pinned

// Energy for the fluid-solid interface is not evaluated since it is not allowed to change

// similar to catbody.fe

$/ /$ parameter contact_angle $=5$ 

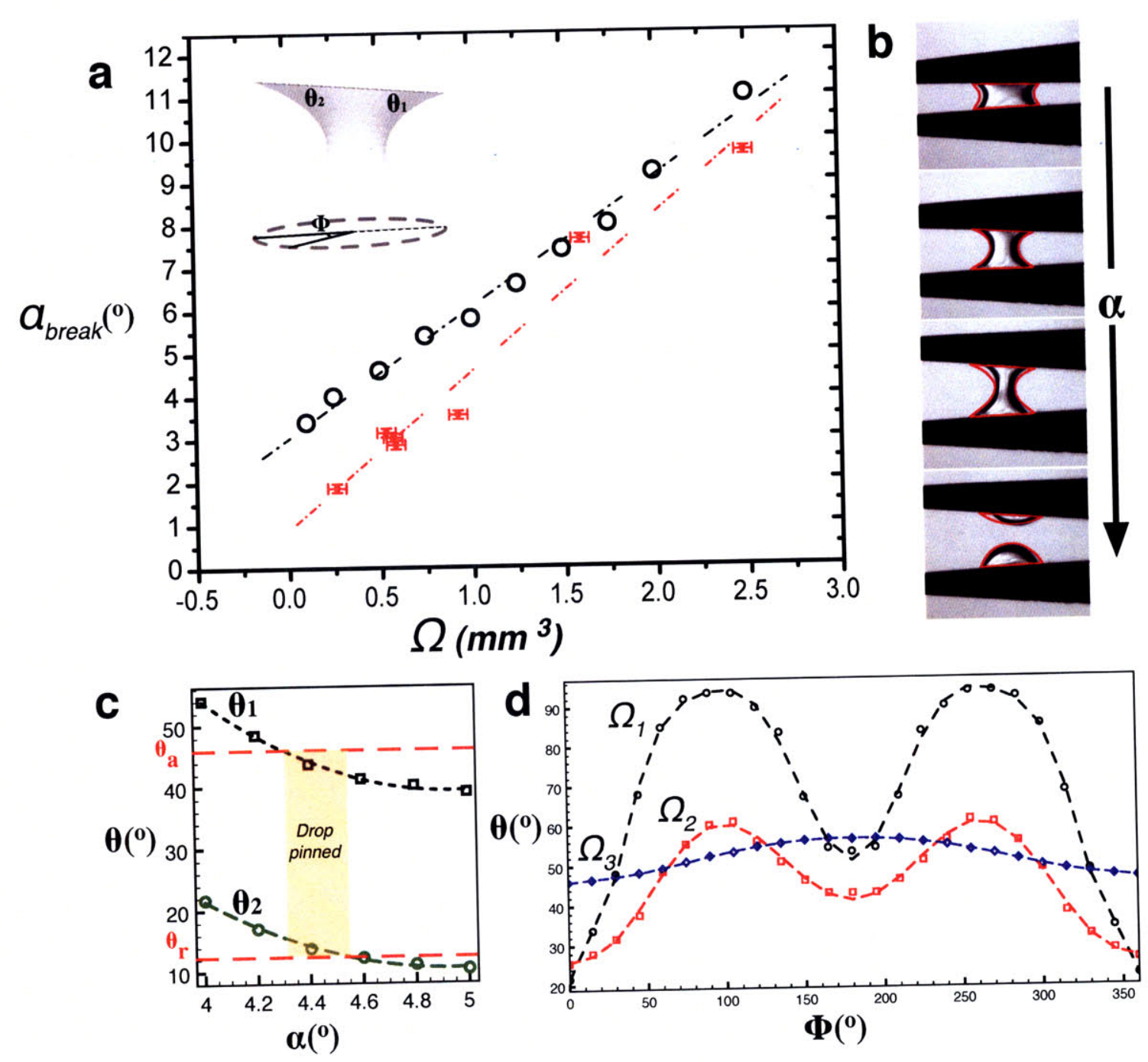

Figure 6-5: A droplet trapped in a wedge (a) Experimental (red squares) and 3D numerical (black circles) evaluation via Surface Evolver ([?]) of the critical angle $\alpha_{c}$ at which a drop of volume $\Omega$ breaks. (b) Quasi-static evolution of the interface of a partially wetting drop trapped between two non-parallel plates with increasing wedge angle $\alpha$. The red curve depicts the numerical simulation derived by minimizing surface energy, which closely matches the observed interface shape. (c) Numerically deduced variation of contact angles $\theta_{1}$ (black curve) and $\theta_{2}$ (green curve) with beak opening angle $\alpha$. Advancing and retreating angles, respectively $\theta_{a}$ and $\theta_{r}$, are marked with dotted red lines. The drop remains pinned provided $\theta_{R}<\theta_{2}<\theta_{1}<\theta_{A}$, corresponding to the shaded region. (d) Variation of contact angle along the perimeter of an asymmetric liquid bridge for varying droplet volumes $\Omega_{1}=2 \mu l, \Omega_{2}=1.5 \mu l, \Omega_{3}=1 \mu l$. The model beak has a width $W=1 \mathrm{~mm}$, length $L_{b}=1.5 \mathrm{~cm}$ and fixed opening angle $\alpha=4^{\circ}$. 
// Contact line modelled as a elliptical boundary on both the top and bottom mandible // with major and minor axis given by $a$ and $b$

$/ /$ parameter major_a $=0.188$

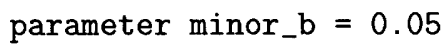

// Beak length and width is only for display purposes. This is not used in determining the location of the drop //parameter Blength= $2 / /$ Length of the beak from apex to vertex //parameter Bwidth=1 // Width of the beak with sharp corners

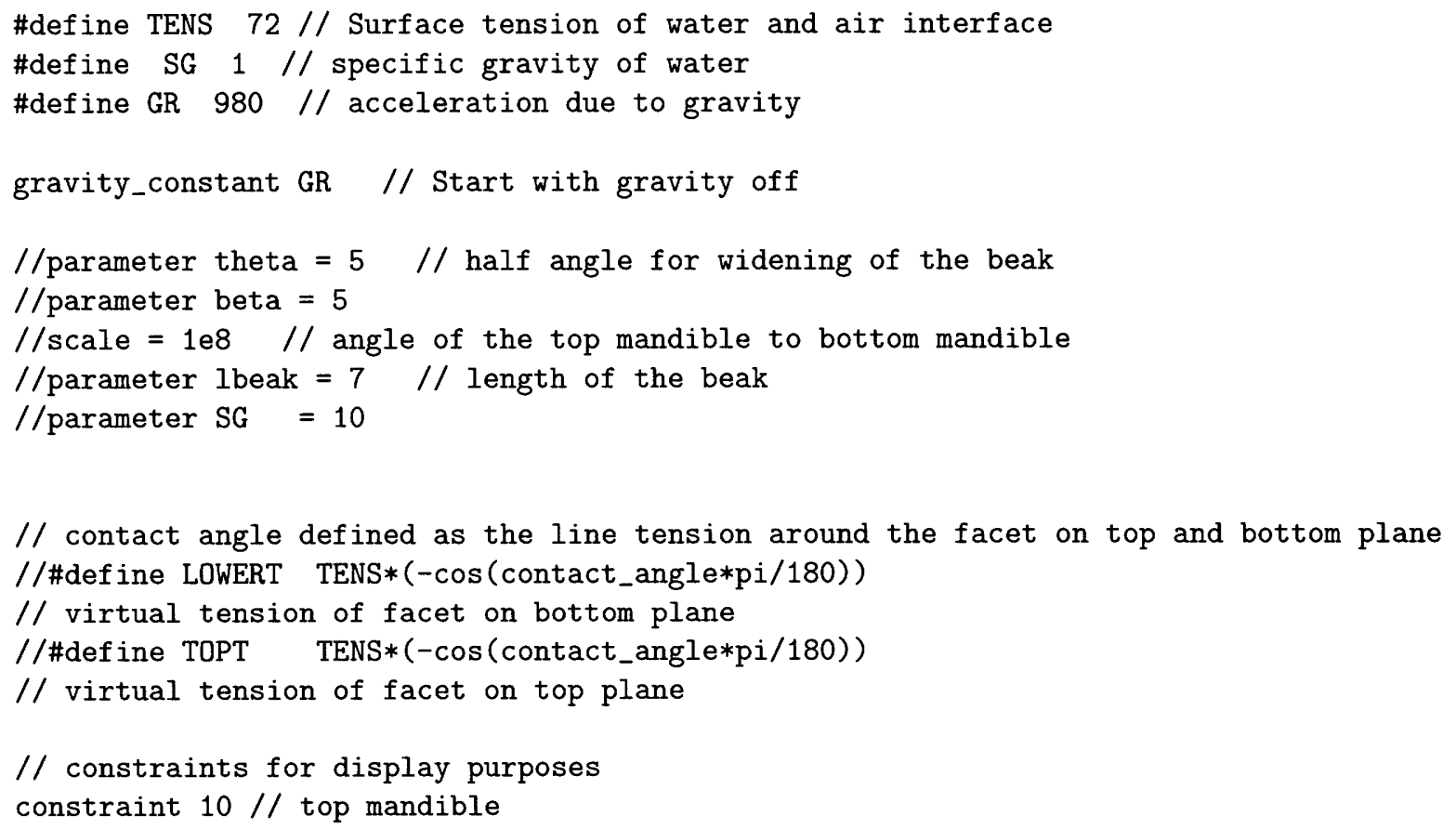




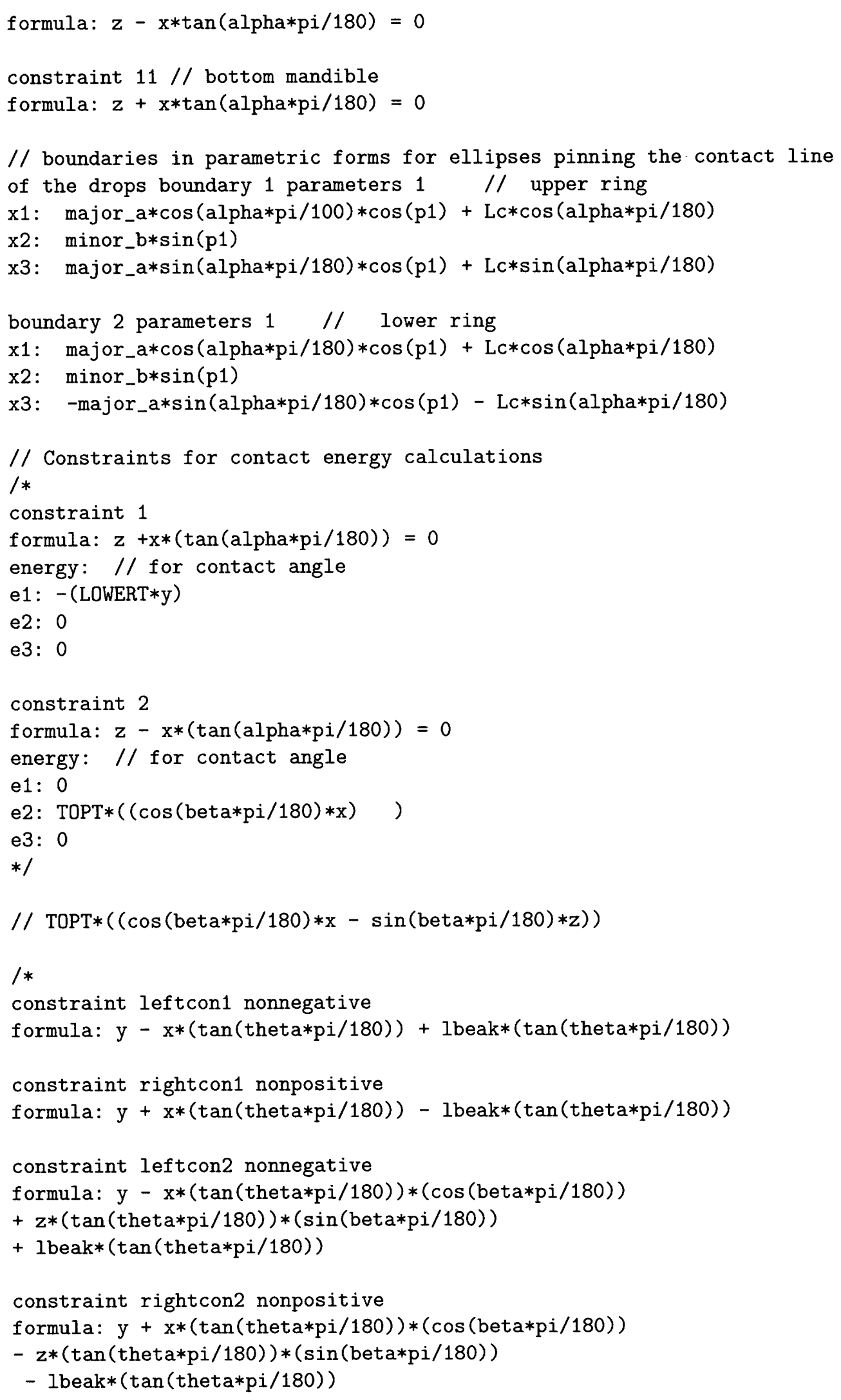




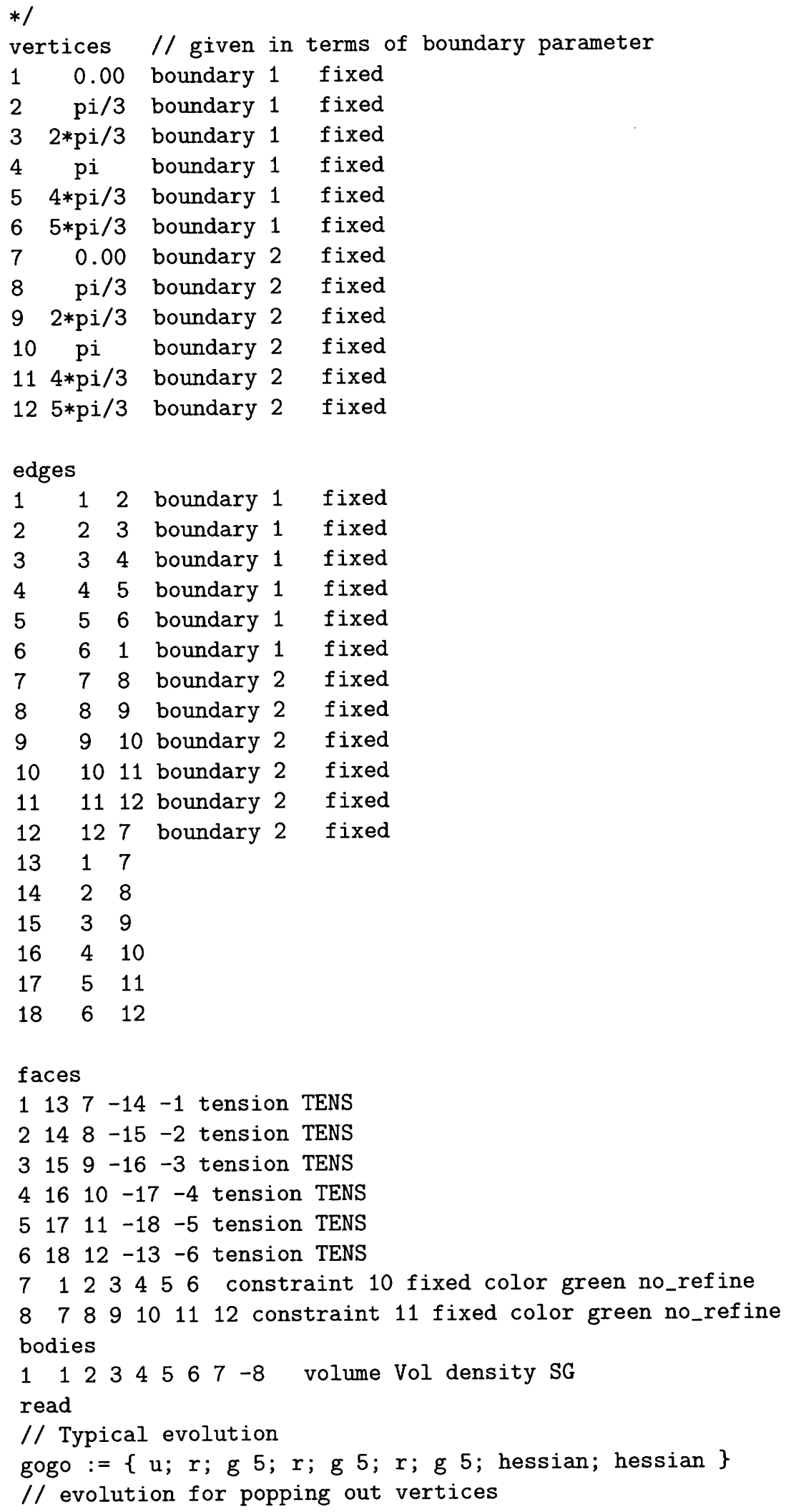




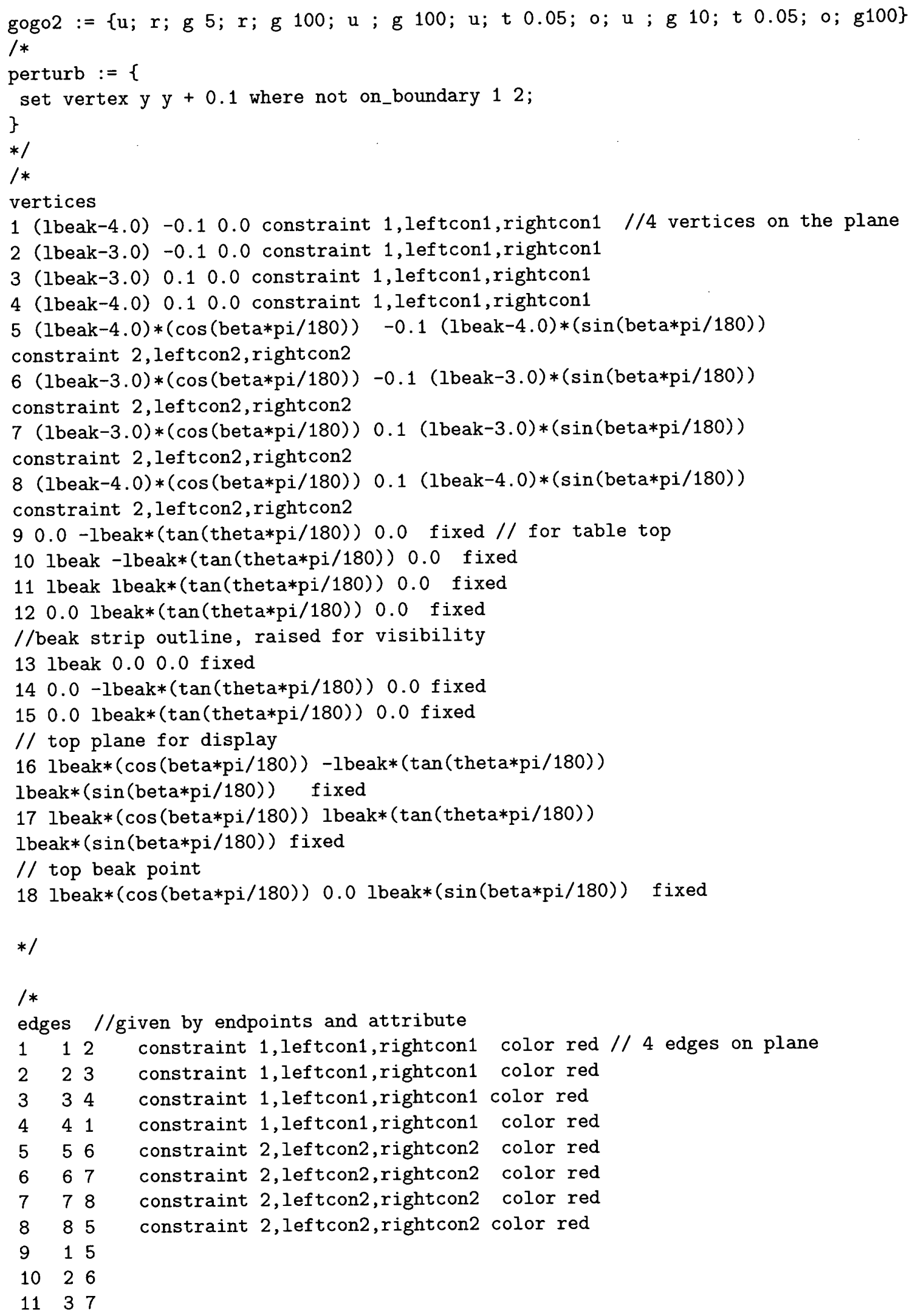




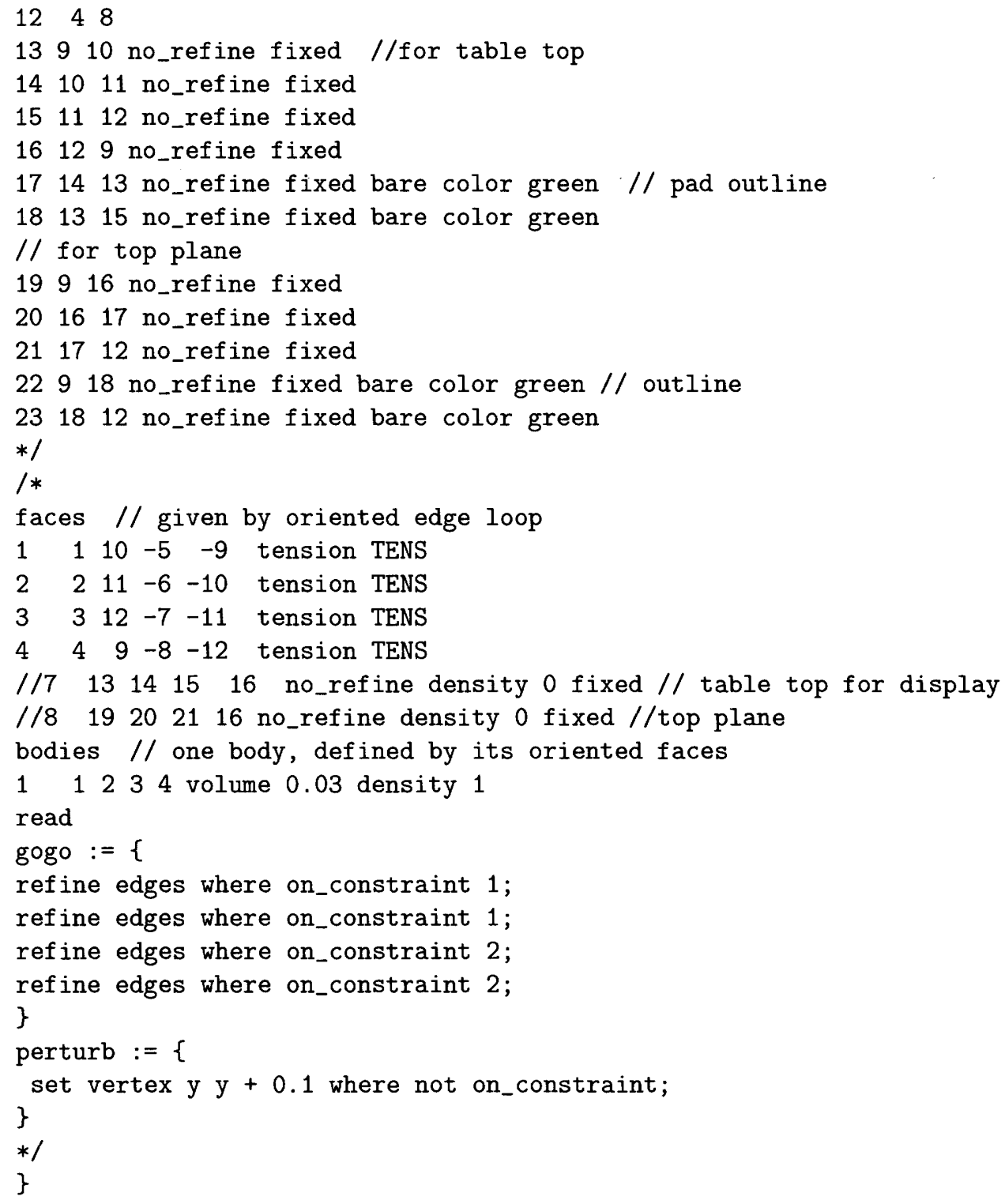




\section{Bibliography}

[1] Andres Barbosa and Eulalia Moreno. Evolution of foraging strategies in shorebirds: an ecomorphological approach. The Auk, 116:712-723, 1999.

[2] H. Bousasse. Capillarité phénomènes superficiels. Librairie Delagrave, Paris, 1924.

[3] F. Brochard. Motions of droplets on solid surfaces induced by chemical or thermal gradients. Langmuir, 5:432-438, 1989.

[4] T Y Chen, J Tsamopoulos, and R J Good. Capillary bridge between parallel and non-parallel surfaces and their stability. J. Colloid Int. Sci., 151:49-69, 1992.

[5] Paul Concus and Robert Finn. Discontinuous behaviour of liquids between parallel and tilted plates. Phys. Fluids, 10:39-43, 1998.

[6] Susan Daniel and Manoj K. Chaudhury. Rectified motion of liquid drops on gradient surfaces induced by vibration. Langmuir, 18:3404-3407, 2002.

[7] Susan Daniel, Manoj K. Chaudhury, and P.-G. de Gennes. Vibration-actuated drop motion on surfaces for batch microfluidic processes. Langmuir, 21:4240-4248, 2005.

[8] P G de Gennes. Wetting: statics and dynamics. Rev. Mod. Phys., 57:827-863, 1985.

[9] P G de Gennes, F Brochard-Wyart, and D. Quéré. Capillarity and Wetting Phenomena: Drops, Bubbles, Pearls and Waves. Springer-Verlag, Berlin, 2003.

[10] R H Dettre and R E Johnson. Contact Angle, Wettability, and Adhesion, volume 43 of Adv. Chem. Ser. American Chemical Society, Washington, DC, 1964. 
[11] E.B. Dussan and R T P Chow. On the ability of drops or bubbles to stick to nonhorizontal surfaces of solids. Journal of Fluid Mechanics, 137:1-29, 1983.

[12] Robert W. Elner, Peter G. Beninger, Daniel L. Jackson, and Tracy M. Potter. Evidence of a new feeding mode in Western Sandpiper (Calidris mauri) and Dunlin (Calidris alpina) based on bill and tongue morphology and ultrastructure. Marine Biology, 146:1223-1234, 2005.

[13] Zoe A. Eppley and M A. Rubega. Indirect effects of an oil spill. Nature, 340:513, 1989.

[14] Sora M. Estrella and Jose A. Masero. The use of distal rhynchokinesis by birds feeding in water. J. Exp. Biol., 210:3757-3762, 2007.

[15] Sora M. Estrella, Jose A. Masero, and Alejandro Perez-Hurtado. Small-prey profitability: Field analysis of shorebirds use of surface tension of water to transport prey. $A u k$, $124,2007$.

[16] Fr. Hauksbee. An account of an experiment touching the direction of a drop of oil of oranges, between two glass planes, towards any side of them that is nearest press'd together. Phil. Trans., 27:395-396, 1712.

[17] Stuart Humphries, Richard H. C. Bonser, Mark P. Witton, and David M. Martill. Did Pterosaurs feed by skimming? Physical modeling and anatomical evaluation of an unusual feeding method. PLS Biology, 5:0001-0009, 2007.

[18] Y. K. Kamath, C. J. Dansizer, and H.D. Weigmann. Wetting behavior of human hair fibers. J. Appl. Polymer Sci., 22:2295-2306, 1978.

[19] Brian James Lowry and Paul H. Steen. Capillary surfaces: stability from families of equilibria with application to the liquid bridge. Proc. Roy. Soc. London. A, 449:411$439,1995$.

[20] S Nebel, D.L. Jackson, and R.W. Elner. Functional association of bill morphology and foraging behaviour in Calidrid Sandpipers. Animal Biology, 55:235-243, 2005.

[21] Bryan S. Obst, William M. Hamner, Peggy Hamner, Eric Wolanski, M A. Rubega, and Bates Littlehales. Kinematics of Phalarope spinning. Nature, 384:121, 1996. 
[22] Manu Prakash and Neil Gershenfeld. Microfluidic bubble logic. Science, 315:832-835, 2007 .

[23] M A. Rubega. The functional morphology and ecology of feeding in Phalaropus lobatus, the Red-necked Phalarope. PhD thesis, University of California, Irvine, 1993.

[24] M A. Rubega. Sexual size dimorphism in Red-necked Phalaropes and functional significance of nonsexual bill structure variation for feeding performance. J. Morphol., 228:45-60, 1996.

[25] M A. Rubega. Surface tension prey transport in shorebirds : how widespread is it? Ibis, 139:488-493, 1997.

[26] M A. Rubega and B S. Obst. Surface-tension feeding in phalaropes: Discovery of a novel feeding mechanism. The Auk, 110:169-178, 1993.

[27] R Stephenson. Effects of oil and other surface-active organic pollutants on aquatic birds. Environmental Conservation, 24:121-129, 2002.

[28] Tamas Szekely, Robert P. Freckleton, and John D. Reynolds. Sexual selection explains Rensch's rule of size dimorphism in shorebirds. Proc. Nat. Acad. Sci. U.S.A., 101:12224-12227, 2004.

[29] Gart Zweers. Transformation of avian feeding mechanisms: A deductive method. Acta Biotheoretica, 39:15-36, 1991. 


\section{Chapter 7}

\section{Future work and conclusion}

In this section, I cover a number of current and future applications of low Reynolds number, all-fluidic logic and control. Throughout this chapter, importance of utilizing physical hydrodynamics of complex fluids in confined geometries is highlighted. I conclude with broader implications of bubble logic in connecting digital fabrication and computation.

\subsection{Advantages}

Hydrodynamics of particles, droplets and bubbles in mico-channel geometries is utilized to build all-fluidic logic and control systems. Microfluidic control technologies developed in this thesis have several unique advantages. No external components such as solenoid valves are required for operation of bubble logic based devices. Analogous to micro-electronics based digital logic family, bubble logic exhibits non-linearity, gain, feedback, cascadability, bistability and error-correction. The system is designed to be modular, thus components can be added together to build more complex control structures - in a hierarchal manner. The device physics is amenable to $\mathrm{kHz}$ range where individual control gates exhibit milli-second dynamics. The devices can be built using any given material set that can be micro-fabricated with a tolerance of 5 to 10 microns. Thus microfluidic systems can be prototyped using elastomeric compounds but can also be manufactured in materials including glass, plastics 
or silicon, with no further design changes. A wide range of materials for device fabrication allows for the use of organic solvents as chemical reagents which would otherwise swell elastomeric materials. The devices can thus be used to study bio-molecules which would otherwise get absorbed in porous channel walls for commonly used elastomeric materials.

Lab-on-chip technologies - a phrase commonly used for miniaturized instruments with integrated fluid processing and analysis; are finding a myriad array of applications in the field of chemistry (kinetics, organic and in-organic synthesis), high throughput screening (drug discovery), biotechnology (genomics and proteomics), material science and single cell and single molecule biology. My work focuses on control technologies for multi-phase microfluidics and has resulted in the development of a new toolbox for sub-nanoliter fluid manipulation in microfluidic systems. Here I briefly present several control architectures that are enabled by bubble logic and discuss near and long term applications in various fields.

\subsection{Combinatorial chemistry}

Means for rapid synthesis of structurally similar compounds has revolutionized the practice of industrial chemistry with applications in pharmaceuticals (drug discovery), material science and polymer science. Medicinal chemists routinely produce libraries as large as 100,000 similar but structurally different compounds. This task is primarily achieved by industrial scale liquid-handling robots. More recently, material scientists have applied the techniques of combinatorial chemistry for the synthesis of new materials.

High throughput liquid-phase synthesis of a combinatorial library is challenging and time consuming for several reasons. Firstly, depending on the number of points of diversity in a library, a very large number of steps can be involved in producing a library. Since all possible combinations of variations are produced in a single shot, a large number of mix and sort operations are involved with many input reagents and reaction conditions. Furthermore, since the output compounds are unique - each compound needs to be tracked and labeled at every stage of the synthesis. 
Current methods for combinatorial synthesis can be divided into static (surface bound) and dynamic (continuous stream). Static methods produce a combinatorial output of chemical species by the arrangement of input lines from two library of compounds. By washing an activated surface by a series of compounds, a static library of compounds can be constructed. The scheme is primarily used for solid-phase synthesis where the final substrate is bound to the solid surface of the device. Thus a set of $m+n$ input lines bring the reagents and $a$ cross-bar network results in all possible combination $(\mathrm{m} \times \mathrm{n}$ ) of the generic type $(\mathrm{m}, \mathrm{n})$ (see figure 7-1). A microfluidic version of this scheme was implemented by Ismagilov et al. [10], where a binding assay between enzymes and immobilized substrates was performed using a diffusion barrier between two layers of microfluidic channels (see figure 7-1a). Enzymes were flown in a set of vertical channels with substrates flown in a horizontal array. A porous membrane separates the two layers where the product is formed at the intersection of the two lines.

Schematic of a continuous, liquid-phase combinatorial synthesis is shown in figure 7-1 (b). The scheme allows for a continuous production of all possible combinations of products from a given set of reagents. For single-phase flow such a scheme can be miniaturized with a series of three dimensional channels where products are formed at the junction of two channels containing the required products. A microfluidic versions of continuous flow combinatorial processors has been constructed for a $2 \times 2$ reaction array [11]. The implementation relies of multi-layer channels with inter-connecting vias between channels to accommodate for channel cross-overs.

In order to improve the throughput of a combinatorial chemical processors, it is advantageous to use multi-phase flow with droplets of distinct reagents fused in the right combination. Compartmentalization of individual compounds into liposomes or nano-liter droplets has been previously proposed. For building a combinatorial library of parts, manual combinatorial fusion of liposome libraries was achieved [21] via sequential fusion of liposomes. This resulted in a complex library of liposomes with a combinatorial content library. Since this was achieved manually using a micro-mechanical gripper, only several combinations can be created. See figure 7-2 

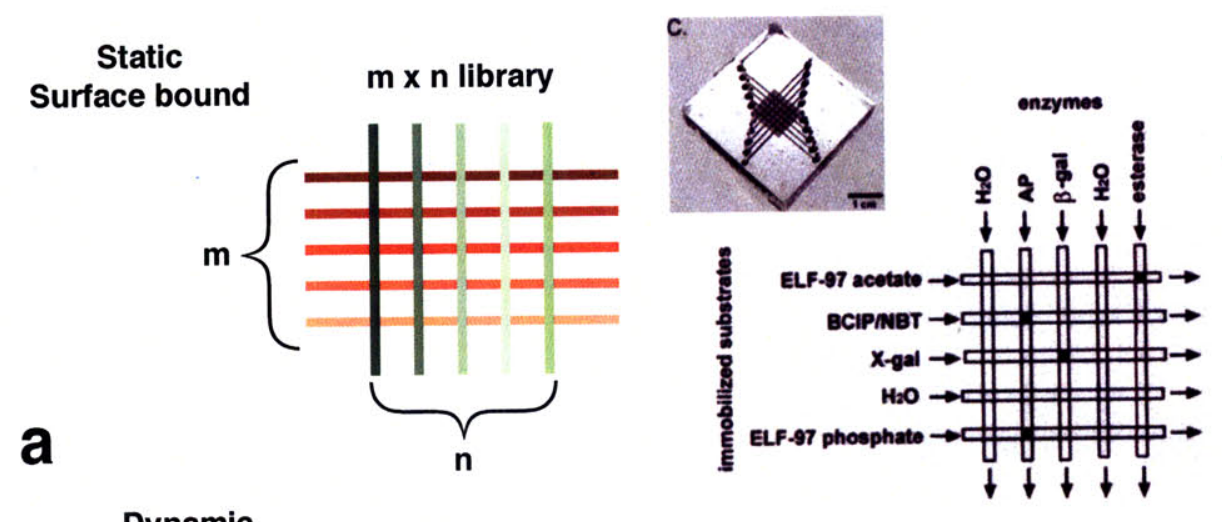

\section{Continuous-phase}

\section{b}
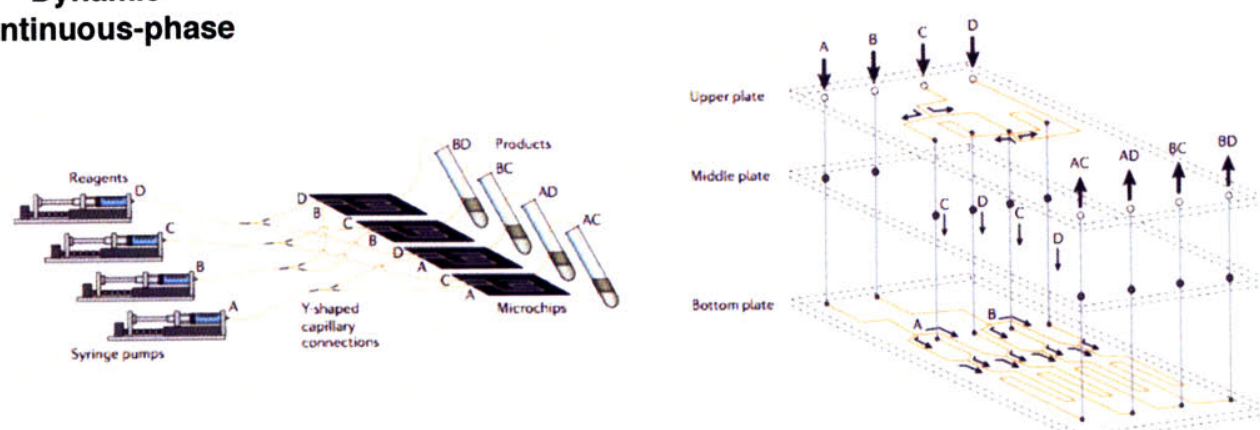

Figure 7-1: Static and dynamic generation of combinatorial libraries (a) Cross-wire microchannels separated by a diffusion barrier is utilized to build a static combinatorial library. Image adapted from [10]. (b) A continuous-phase combinatorial generation of reagents in a microfluidic manifold. Image adapted from [11]
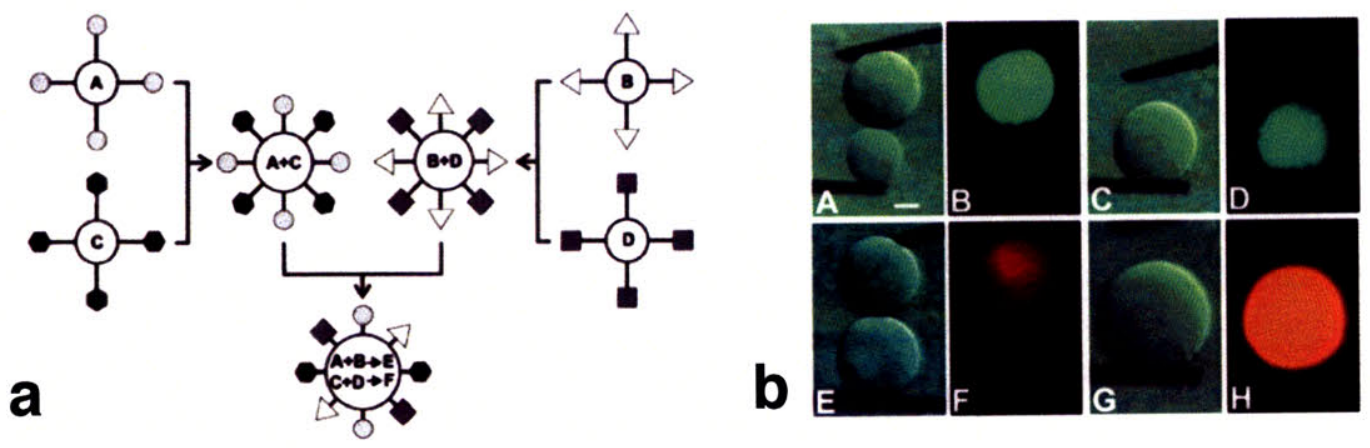

Figure 7-2: Manual synthesis of combinatorial liposome library with liposomes fused via micro-manipulators under manual control. Figure adapted from [21]. 
Rapid, continuous synthesis of a large combinatorial library using individual nano-liter droplets in a miniature device is a formidable challenge and has not been solved as yet. Since the number of input, control and output lines for a $\mathrm{m} \mathrm{x} \mathrm{n}$ library grows exponentially, the network topology for a simple combinatorial assay becomes extremely complicated. Here I propose (see figure 7-3) a droplet based scheme for building a combinatorial library of compounds in a continuous fashion. The task of skillful routing, mixing and sorting is handled using fluidic logic. Thus no external control line is required for the synthesis. The scheme allows for continuous generation of a library. The generated sequence of compounds can be stored or directly utilized after production in a screening assay. Moreover, since the individual library components can be sorted, further analysis is feasible after synthesis. Such a modular, general purpose combinatorial chip would find direct applications in medicinal chemistry, specifically for the synthesis of small compound libraries. Together with a scheme for microfluidic chemical memory (see next section on dynamic micro-arrays), the synthesis chip presented here provides a powerful set of tools for combinatorial chemistry.

\subsection{Dynamic Micro-arrays}

Micro-arrays provide a high-throughput means of exposing a large population of bio-molecules like DNA strands and proteins to controlled chemical and biological environments. An important criteria guiding the development of micro-arrays over the years has been smaller spot size - allowing for testing a large set of conditions in a single experiment. The technique has recently been generalized for construction of single cells arrays on chip. Single cell based micro-array, which involves trapping a large number of a cells on a defined grid pattern, has recently been established as a powerful technique for single cell toxicity studies and whole cell response to drug molecules $[25,26,12]$.

Post-analysis of species on a conventional static micro-array platform is usually very limited. Several experiments employing optical traps [12] have been used previously to capture and dislodge targeted locations on a micro-array - without harming the biological entity. Such an integrated capability of dynamic manipulation of micro-array content would allow for 


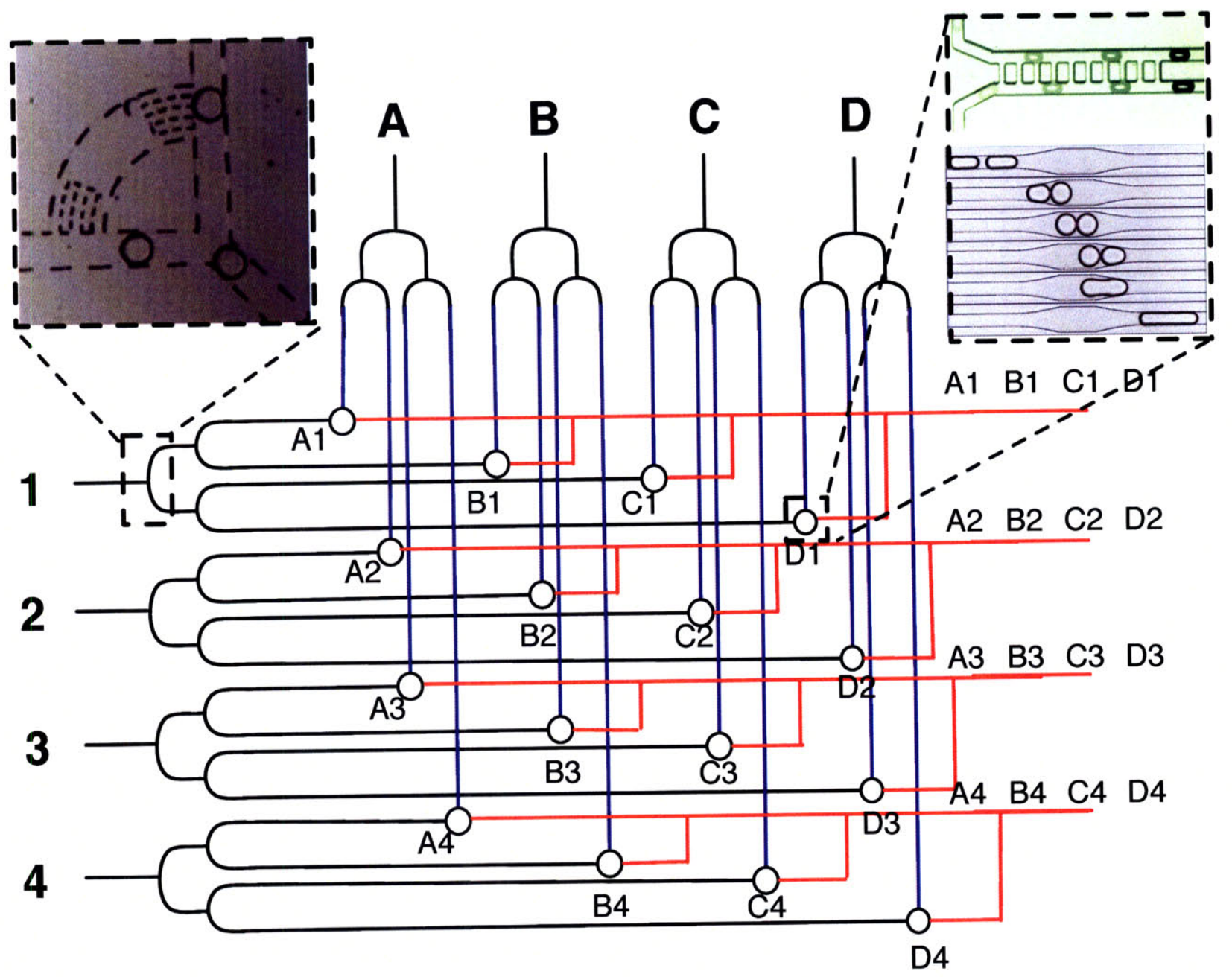

Figure 7-3: Schematic of an all-fluidic droplet combinatorial processor with 8 input lines, 4 output lines and no control lines for continuous production of a library of $4 \times 4$ compounds with integrated modules for logical routing, fusion and synchronization. 


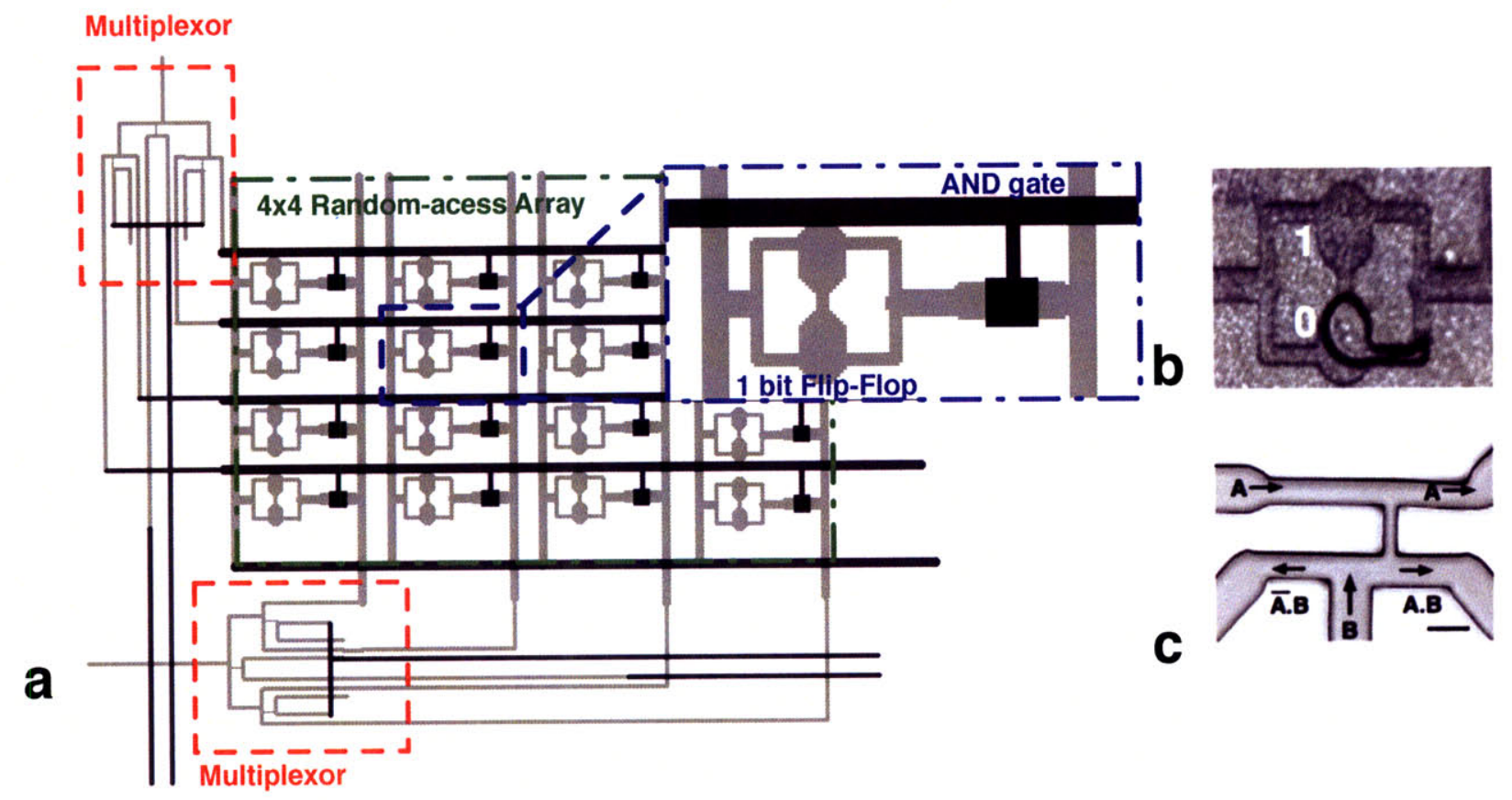

Figure 7-4: (a) Schematic layout for a random access chemical memory for storage and retrieval of sub-nanoliter fluid droplets depicting multiplexors for control and flip-flop array for storage. (b) Microphotograph of a one-bit flip-flop. (c) Microphotograph of AND/NOT logic gate.

multi-step screens in a high-throughput, micro-array format. Previous attempts for developing dynamic-microarrays have involved complex optical [26, 25, 12] or di-electrophoretic [23] setups integrated on the micro-array surface. In this section I propose plans for developing an all-fluidic scheme for implementation of dynamic-microarrays with the ability to programmatically trap and release fluid droplets, bubbles and solid particles.

Previously, I reported [16] a hydro-dynamic trapping mechanism for purely fluidic storage and retrieval of droplets and particles in a bistable flip-flop geometry. A large array of fluidic memory units can be integrated to develop an all-fluidic dynamic micro-arrays. This allows for hydrodynamic storage and retrieval of droplets and particles (single cells, encapsulated droplets). The mechanism allows for working with both adherent (which are usually bound to the micro-array surface in case of static micro-arrays) and non-adherent cells via encapsulation techniques. The concept relies on a bistable energy trap where fluid flow in a given geometry creates a stable trapping mechanism for a single particle. Arrival 
of another particle to the trap, dislodges the current particle providing a simple mechanism for recovering a specific particle at a given location. For integrated addressing schemes for these arrays, a series of multiplexors are used.

Large scale compound libraries (of the order of a million compounds and higher) are conventionally stored and managed using large robots in pharmaceutical settings (also see chapter 1). These platforms are fairly cumbersome and expensive. An integrated droplet storage and retrieval scheme can be scaled to large integrated arrays for random-access chemical compound memories (Chemical-RAM). This would be a significant improvement over current means of storing artificial or natural compound libraries and reduce the cost of maintaining and adding such libraries. Furthermore, compound libraries could be used with integrated analytical instruments in industrial settings without the infra-structure needed to run large scale compound storage solutions.

\subsection{Surface bound detection and analysis systems}

In previous sections, I have only discussed schemes that use individual droplets and bubbles as a micro-reactor - where the reagents are confined inside micro-droplets. In this case, all reactants are mobile and traverses the microchannels. This scheme does not utilize the static surface of the channel walls - that could also provide a reaction site. Here I describe a new device configuration where stimulants are carried by individual droplets while the substrate of interest is bound to the channel surface. This provides us with an opportunity to stimulate the substrate at a varying flow-rate, concentration and frequency. Such a programmable means of applying a stimulus to a substrate opens up new avenues in probing biological and chemical systems with a complex stimulus profile allowing us to study frequency response of biological parts.

I further summarize the idea of surface bound microfluidic detection via a proposed scheme for odorant detection employing olfactory receptors. 


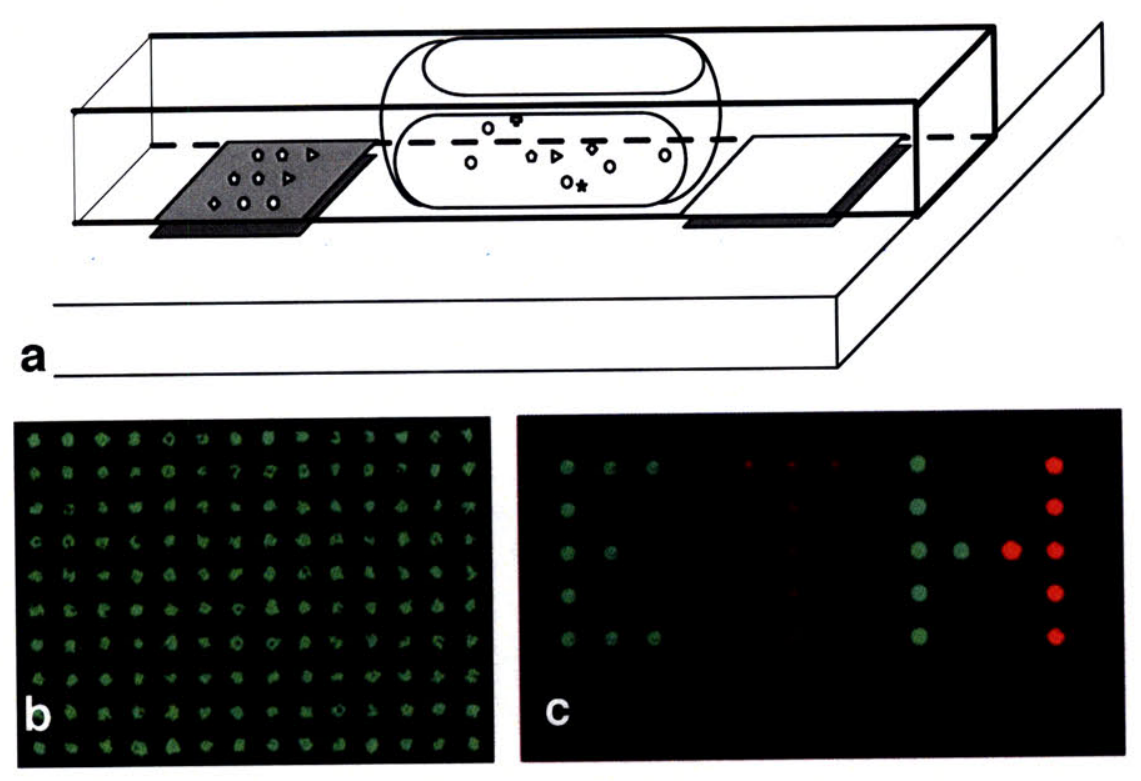

Figure 7-5: Frequency controlled stimulation of surface bound substrates using droplets and bubbles. (a,b) Cell and vesicle micro-arrays bound to the chip surface. Image of cell micro array from [5], vesicle array from [20]. (c,d) Schematic of droplets traversing a microchannel network carrying stimulant as cargo.

\subsubsection{Microfluidic Olfactory receptor based odorant detection}

The nasal cavity is a highly evolved structure that transports odorants efficiently, allows temporal signal modulation via sniffing, offers a large surface area for detection and also conditions air for breathing. Full 3D morphological data has only recently become available for nasal cavities of dogs [4] and other animals, revealing several findings critical to the design of an artificial nose including extremely high surface area for odorant binding and heat transfer, laminar flow and complex by-pass regions for tunable airflow control. Here I propose an integrated microfluidic "RealNose" design for odorant intake based on the programmable generation and control of sub-nano-liter gas bubbles in a two-phase microfluidic chip. This system, while at first glance very dissimilar to the canine nasal cavity in fact effectively simulates the function of both the morphology of the biological nose and the mucous layer. Furthermore, it allows for "physics based signal conditioning by intelligently addressing the odorant carrying bubbles to immobilized OR sites. By integrating fluid and gas delivery on a single on-chip platform in a multi-phase system, it will be possible to transport and replenishment of chemical reagents, on-chip synthesis of olfactory receptors 


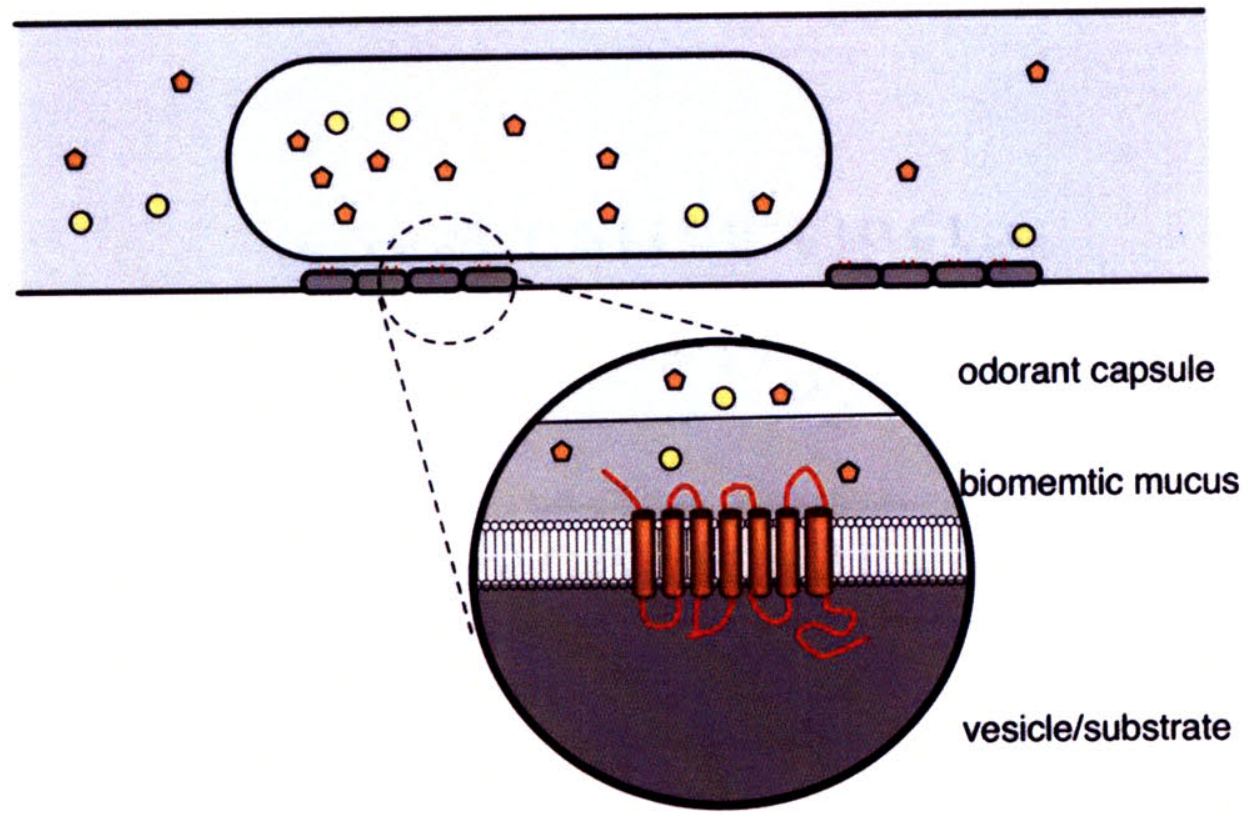

Figure 7-6: Mechanics of odorant transport from bubbles to olfactory receptors on the chip surface. The thin surrounding liquid film effectively acts as an artificial mucus (see inset with odorant molecules depicted in color).

and time-modulated and programmable delivery of odorant molecules in a highly integrated fashion.

For an air bubble containing odorants traversing a thin channel (see figure 7-6), a thin fluid film (sub-micron) forms an artificial mucus layer, always wetting the OR sites. Since odorant molecules are retained in this artificial mucus layer for different times, a spatial and temporal response of odorants can be developed by the detector, allowing chromatographlike segregation of complex odorant mixtures. Such a concentration based impulse response measurement allows for unique time-varying signatures to be characterized for complex odorant mixtures.

Bretherton in the 1960s [6] studied gas bubbles in a wetting fluid traversing small capillaries, analytically predicting the thickness of the sub-micron fluid film surrounding the gas phase. Mozell et al. developed a theoretical model of mass transport for the first stage of odorant capture from air to the olfactory receptors [13] Diffusion of odorant molecules away from the interface in the mucus layer governs the odorant capture rate from the nasal airflow. 
For the particular model of odorant capsules, I will analytically and experimentally study odorant concentration in the artificial mucus layer as a function of intake flow velocity. The analytical modeling efforts will focus on the geometry of odorant capsules utilized in our DARPA RealNose design. With microfluidic control of both odorant bubbles and surrounding fluid, it is possible to modifying $\mathrm{pH}$, ionic concentrations, temperature, pressure and composition of the mucus lining along its entire length. Such flexible control over the

fundamental properties of the mucus layer allows us to vary a number of parameters to study its effect on odorant distribution, something that can not be achieved in a static biological or artificial nose and will allow fine-tuning of parameters depending on the environment in which the RealNose operates (e.g. to compensate for effects of varying humidity, altitude etc.) which will be of particular value to defense applications of a portable RealNose.

I will also explore a novel technique of increasing odorant concentration for high sensitivity detection by introduction of a micro-emulsion in the mucus. Odorant molecules are selectively captured by the emulsion phase [7] resulting in significant odorant concentration in a specific emulsion phase. Via tuning the emulsion size and type, a novel odorant differentiation scheme capable of separating several classes of odorant molecules will also be explored.

\subsubsection{Odorant control for patterned stimulation of olfactory receptors}

Current microfluidics technologies are dependent on external macro-scale control elements, thus requiring a lab full of components to run the chip. I have invented a new paradigm in flow control, Bubble Logic [16] that allows programmable control of gas or liquid bubbles in micro-channel network via all-fluidic means. Bubble logic provides a droplet-level, internal, inherently digital flow control mechanism at $\mathrm{KHz}$ frequencies with no moving parts or off-chip components particularly suitable for a portable and sturdy RealNose capable of withstanding vibration and environmental changes. Nonlinearity is introduced in an otherwise linear, reversible, low Reynolds number flow via bubble-to-bubble hydrodynamic interactions. Members of our team have demonstrated bubble logic AND/OR/NOT gates, a toggle flip-flop, a ripple counter, a timing restoration device, a ring oscillator, a bistable 
valve and an on-demand bubble generator [16]. These show the nonlinearity, gain, bistability, synchronization, cascadability, feedback and programmability required for scalable universal computation and control.

Furthermore, I propose a fractal-like channel geometry to capture air samples from a large area for improving the sensitivity of the device. This allows for a device that can be scaled to large areas in the future, for instance to be integrated into soldier clothing, tents, walls, fences etc. Using this design, the RealNose will be capable of selectively probing specific regions of the sensor surface on demand and for large enough surfaces the RealNose could provide directional information (i.e. point to the source of the odorants) without needing to move (unlike a trained canine).

Above I describe two representative geometries for odorant intake (red arrows mark flow of odorant capsule). The entire device requires only a single source of negative pressure that can be obtained from a micro-fabricated vacuum pump. This alleviates a number of external control elements significantly reducing the size and cost of the proposed device and adds durability and ruggedness to the device.

\subsubsection{Artificial Sniffing}

The role of sniffing in olfactory processing is thought to significantly improve the odorant detection limits in mammalian noses via improved deposition of odorant molecules to the receptors and by playing a role in odor encoding, facilitating coordination of the olfactory system with further neural processing $[1,19]$. Various animals studied have been recorded to sniff at constant frequencies (the canine at 5-6 Hz). Verhagen et al. recently demonstrated sniff frequency-dependent filtering of sensory input to the olfactory bulb [22]. Numerical modeling of airflow in rat and human nasal pathways [29] reveals very little difference between turbulent and laminar flow intake in terms of odorant deposition efficiency. Due to high flow resistance of complex nasal fluid pathways, required for increased surface area for olfactory receptor layers, flow inside the channels where odorant transfer to the mucus layer is primarily laminar. 
Time-modulated odorant intake achieves tunable sniffing behavior via a purely fluid dynamic mechanism of bubble formation at a fixed frequency in a microfluidic device. The odorants are captured into nanoliter to microliter capsule bubbles localizing the concentrated odorants allowing for a variable sniffing frequency that is tunable by simply changing the (otherwise constant) value of the negative pressure driving the flow from a single source, yielding frequencies from $\mathrm{Hz}$ to $\mathrm{kHz}$ regime without the need for variable pressure pulses as in the biological nose. This again reduces the number of moving parts and makes the device suitable for miniaturization and real-world operation and even carries the promise of surpassing the biological nose by increasing the effective frequency of sniffing from $\mathrm{Hz}$ to $\mathrm{kHz}$.

\subsection{Microfluidic Lock-in amplifier}

A commonly used technique to improve the Signal to Noise Ratio (SNR) ratio is phase sensitive detection via modulation of the excitation source at a given frequency. For fluorescence measurements this is achieved via modulation of the excitation frequency. Electronic lock-in amplifiers have been extensively used for detecting time varying signals in noisy environments. The technique utilizes an in-phase excitation signal and a reference signal to cancel out the noise out of band. Thus very sensitive detection can be achieved, even for conditions where the noise significantly dwarfs the signal.

Previously, micro-rotors made of magnetic chains of particles or magnetic janus particles have been utilized to enhance signal-to-noise ratio of detection schemes using a characterized input signal. Anker et al. [2,3] proposed rotating asymmetric magnetic janus particles that can be excited to a fixed angular velocity using an external driving field, thus modulating the fluorescence signal form the probe. Vuppu et al. [24] used a similar idea to build a rotating paramagnetic microsphere chains to improve sensitivity of a solid-phase immunoassay.

Here I propose a lock-in detection technique that utilizes high frequency dynamics $(\sim \mathrm{kHz})$ of droplets and bubbles carrying an active stimulus. I present the idea in context of a signal transduction scheme for olfactory receptors but note that this is a generic technique that 
can be applied systems equally well. The scheme improves the signal-to-noise ratio of a time varying components of the signal.

It is important to improve the sensitivity of OR detection to obtain reliable results for complex and low concentration odorant mixtures. Unmodulated background signal (noise) can significantly reduce the sensitivity of measurements. "Microfluidic Lock-in Detection can significantly improve the signal to noise ratio (SNR) for odorant sensing applications via phase-sensitive detection. The implementation is highly integrated into our odorant intake technique, thus requiring minimal components. A microfluidic lock-in detection technique will be developed where odorant samples will be introduced to the detector at a known frequency $(\mathrm{Hz}$ to $\mathrm{KHz}$ range) inducing a periodic signal response from the detector. Since this response frequency directly corresponds to the frequency of sample introduction (arrival frequency of odorant bubbles) an off-the shelf lock-in detector can be used to remove noise and improve SNR. Several distinct advantages of the proposed scheme include very low-concentration target odorant molecule detection via improved signal-to-noise ratio and enhanced micro-mixing induced by bubble motion in both liquid and gaseous phase.

Figure 7-7 describes one arrangement for implementing a phase sensitive microfluidic lock-in odorant detection. The scheme is applicable to any real-time odorant detector employed, several of which are described in other parts of this proposal. A programmable bubble generator is used to form periodic odorant capsule (bubble) at a tunable frequency in a micro-channel. The detector signal is fed into an off-the-shelf lock-in amplifier with a reference signal corresponding to the bubble frequency. The input reference signal can be generated via an optical probe chopped by traversing bubbles. I have already shown tunable frequency generation of bubbles in a microfluidic device from several $\mathrm{Hz}$ to $\mathrm{KHz}$ range [16]. For testing purposes, I will implement and demonstrate improved SNR for a fluorescence detection for surface bound molecules. Next I will translate the same detection scheme for the odorant detection for our 


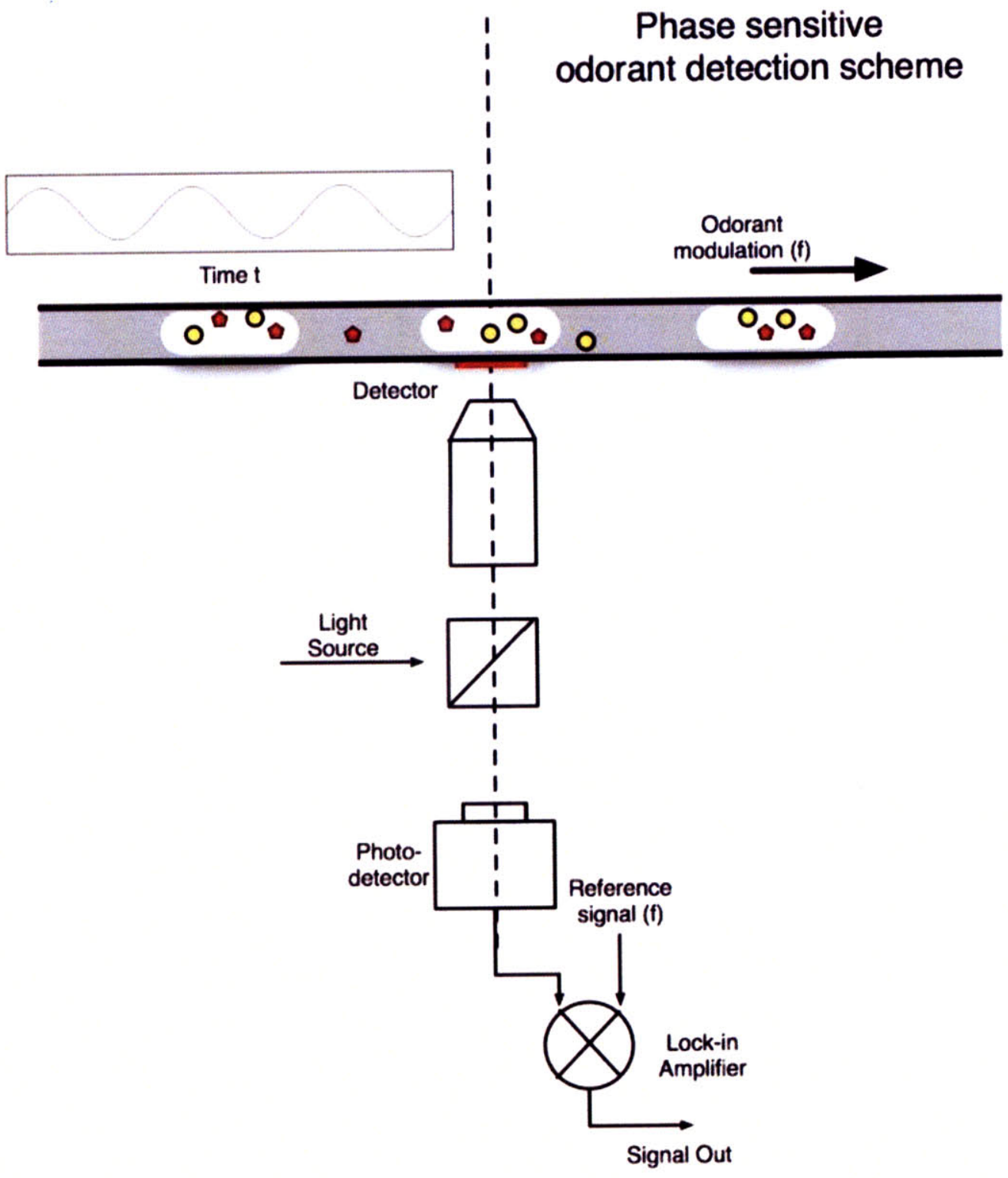

Figure 7-7: Microfluidic lock-in amplifier: phase sensitive detection of a sample via tunable frequency intake. 


\subsection{Micro-droplet processors}

Near term, I believe most immediate benefits of microfluidic logic will be seen in integrated control of micro-droplet processors. As described previously, a vast array of chemistries including polymerase chain reaction (PCR), single molecule PCR, transcription, translation for in vitro protein expression, protein crystallization, single cell handling and so forth (see figure 7-8) has already been ported to droplet platforms.

On the contrary, high throughput control systems for multi-phase microfluidics and droplet processors are still in development phase. Most of the benefits of droplet microfluidics can only be realized with droplet-level, arbitrary control schemes operating at $\mathrm{kHz}$ frequencies. I present one such scheme which exploits the physical fluid dynamics of droplets and bubbles in channel network to implement a completely fluidic and universal control and logic. I believe, immediate applications of fluidic logic in integrating control of nano-liter droplet chemistry, will be most beneficial.

Biological machines are a fascinating example of evolution of optimized machines for very particular tasks, at all length scales. Taking an example of proteins as biological machines; various tasks in chemical systems can be achieved efficiently by employing biological parts, be it chemical synthesis using biological enzymes or natural surfactants. When deployed in engineered systems, chemists face tremendous challenges since biological parts are extremely optimized for natural reaction volumes and reaction conditions. This problem can be solved by employing integrated bio-processing mechanisms that can synthesize, analyze and optimize biological parts in engineered settings.

One such approach involves implementation of in vitro protein evolution and selection strategies, where protein function is evolved by applying an evolution pressure and a screen leading to the selection of optimized molecular structures (that could not be predicted by rational design). An important aspect involved in this strategy involves the capability to isolate monoclonal species and gene libraries allowing amplification of a single mutant gene after the selection step. Droplet based microfluidic devices provide a platform for performing exact metering and dispensing of reagents, in-vitro transcription/translation for enzyme 


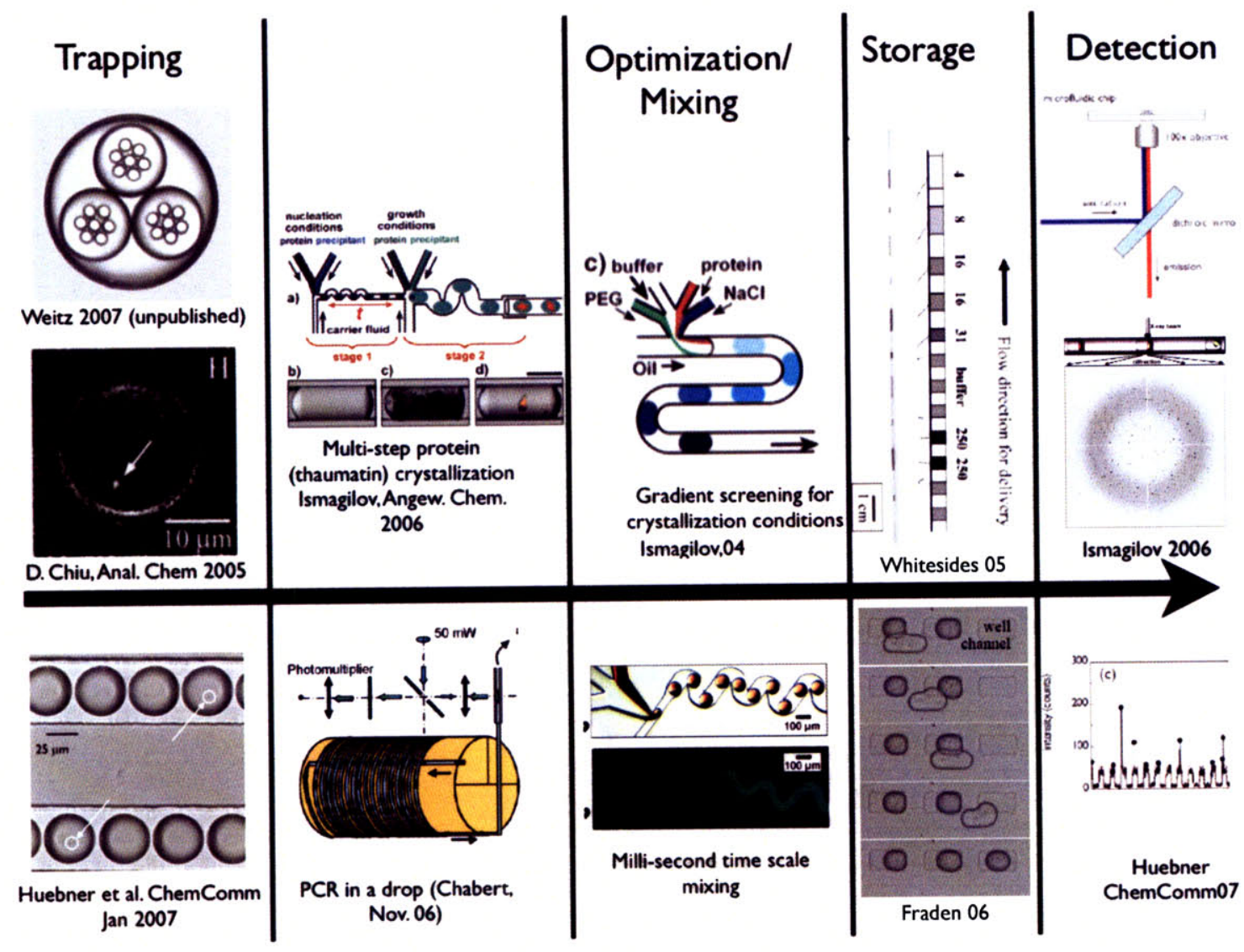

Figure 7-8: Sub-nanoliter chemistry, defined as reactions performed in micron-scale droplets in microfluidic systems. The Image depicts methods including trapping, bio-molecular amplification, optimization, storage and detection of biomolecules. The ability to generate and manipulate sub-nanoliter droplets in a confined geometries coupled with ultra-senstivie inline-detection schemes enable the implementation of high-throughput assays for biological and chemical analysis. 
synthesis, multi-step and parallel reaction chemistries and selection assays and in-band sensitive detection and selection, providing unique advantages inaccessible in other platforms. Water-in-oil emulsions have been previously used to exhibit in-vitro cell-free protein expression in individual droplets [8]. As components, in vitro-expression requires tRNAs, amino-acids, aminoacyl-tRNA-synthetase, ribosomes, initatiation/elongation/releas factors and protein-coding gene sequences; usually available as cell-free expression mixtures. For a droplet size of $5 \mu \mathrm{m}$ droplets, achievable in current microfluidic geometries, a reaction volume as small as $65 \mathrm{fL}$ can be achieved. Implementing multi-step chemistries, variable selection pressure, integrated storage and post-analysis of selected genotypes and phenotypes would mark the next phase of advances in directed-evolution.

The field of synthetic biology is leading to a large number of engineered biological parts being developed for field deployment, similar to other fields in engineering. To benchmark and optimize biological parts in a manner similar to engineered systems, integrated droplet processors capable of online synthesis, analysis and optimization will become necessary.

\subsection{Fluidic control}

Bubble logic may also find a broader set of applications in fluidic control of mechanical systems. Since fluidic logic employs a single operating medium i.e. both the transducer and the control consists of the same medium, integrated actuation and control are possible.

\subsubsection{Soft robotics}

Traditional robotics has employed stiff actuators for locomotion. On the other hand, biological systems are replete with examples of soft actuators, including muscles and hydraulic or fluidic actuators. Spiders and earthworms for e.g. are known to employ internal hydraulic pressure in body cavities for controlled actuation. Most spiders lack muscles at the knee

joint, thus primarily relaying on the internal pressure of a fluid filled cavity (hemolymph) for extension of a given joint . Measurements on living spiders has shown internal pressure 

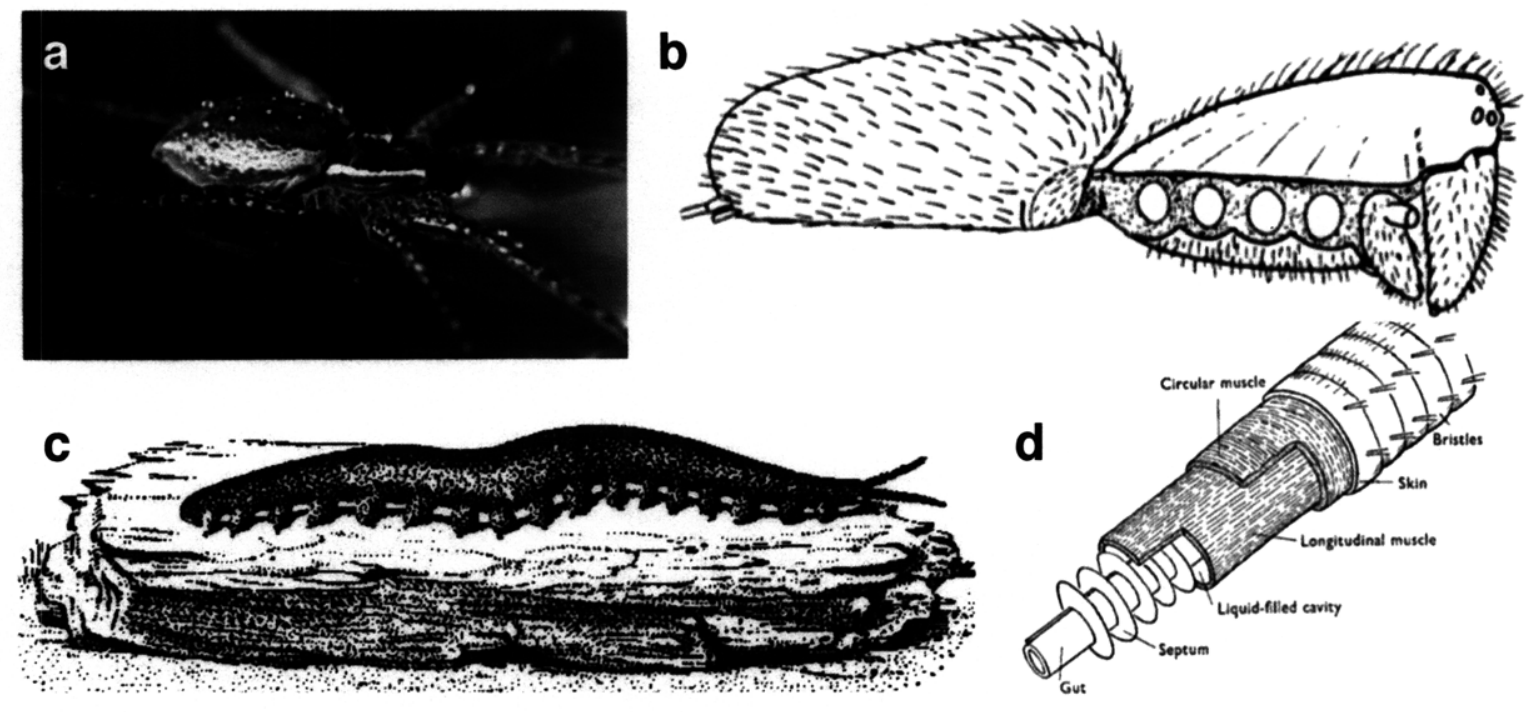

Figure 7-9: Biological machines employing fluidic means of locomotion and control. (a) Photomicrograph of a water spider that employs internal pressure in the leg segment as a means of locomotion. (b) The sketch shows the hydraulic cavity connected to the leg segments. Image from [15] (c) Sketch of a velvet worm [14].(d) Details of a musclo-hydraulic system of an earthworm with surrounding muscles and fluid cavity for locomotion [14].

variation from $5 \mathrm{~mm} \mathrm{Hg}$ to $40 \mathrm{~mm} \mathrm{Hg}$ during a single actuation stroke [15]. Moreover, fluid pumping schemes, such as a heart, are employed to modulate this pressure resulting in an integrated control system for actuation. This provides inspiration for a new paradigm in robotics - called "soft robotics" which aims to build machines that are compliant and exhibit a broad range of morphological deformations.

Fluidic actuators and control systems provide an integrated platform for designing soft robots - where both the actuator and the micro-controller is fabricated using elastomeric materials. Fluidic systems provide an advantage of having a single operating medium for both actuation and control. Such an implementation scheme opens up a large array of design choices with a high degree of integration. The entire robot could be built out of elastomeric elements and hence capable of morphological transformation not possible using conventional robots (for e.g. pass through a very narrow aperture) that employ electronic control and traditional actuation mechanisms such as motors. 


\subsubsection{Multi-material Print heads}

Current printing technologies employ on-demand droplet generation mechanisms using piezo, thermal and acoustic actuation elements. For the case of ink-jet printers, the head is mounted on a mechanical moving stage that is translated precisely on a receiver substrate. Scaling for high-throughput printing thus requires a very large number of integrated printing nozzles on the same cartridge, which are controlled simultaneously. Current printing methods sequentially take a small amount of ink from the ink reservoir and transfer it to the receiving substrate. Thus no chemical pre-processing is possible before the drop is transferred on the substrate.

Bubble logic provides a new way of building microfluidic printing cartridges for highthroughput, multi-material printing. Thus individual droplets to be printed can be routed and rearranged on chip, before being ejected from the nozzle. A print pattern can thus be pre-formed on a bubble logic chip and transferred onto the substrate. Bubble logic also provides the capability to perform complex chemical and material processing on the print head. Thus pre-processing of functional materials and biological samples is possible. Rather than storing a large number of different functional materials, specific formulations can be synthesized on the fly. This also allows for various operations, like tuning the ink concentration or optimizing the ink viscosity. Because of the availability of a very large set of functional materials on the microfluidic chip driving the printing cartridge, it is possible to print electronic or other functional materials.

\subsubsection{Fluidic displays}

Emerging field of optofluidics - at the interface of microfluidics and optics - allows for design of tunable optical devices. Here we present a bubble logic based optofluidic display. Ondemand bubble and droplet generation and control in microfluidic geometries can be used to precisely pattern droplets or bubbles in a serpentine channel (see figure 7-10).

The optical transmission properties of a bubble varies from the surrounding fluid that encloses it. Various optical techniques can thus be used to make all-bubble displays, where 

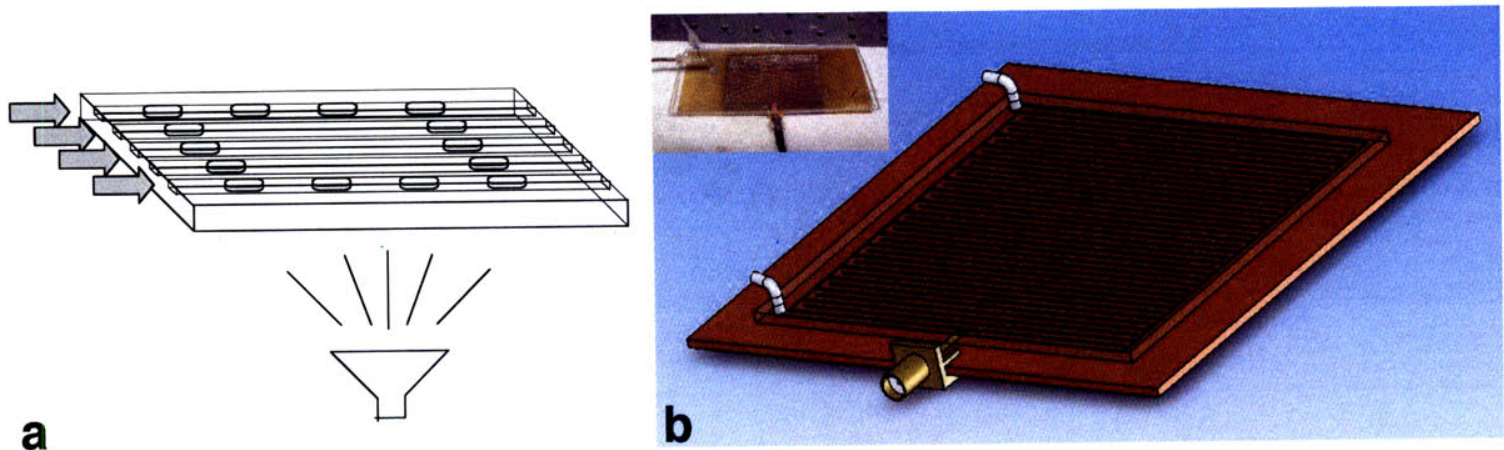

Figure 7-10: Bubble display. (a) Schematic of programmed generation of drops and bubbles in a serpentine channel for a microfluidic display element. (b) Controlled generation of mercury droplets in a fluidic channel for programmable RF components.

a single pixel is represented by the presence or absence of a bubble. Furthermore, using a multi-layer structure, reflective color displays are possible. The bubbles can be further controlled in a bistable array using the previously described bistable gates. This provides basic elements for a fluidic, non-volatile display and projection devices can be formed with no-moving parts. Since bubble logic devices can be manufactured in flexible, plastic substrates by roll-ro-roll manufacturing; bubble logic display provides a promising mechanism to fabricate large area displays.

\subsubsection{Programmable nano-liter drug delivery}

Drug delivery is sometimes as simple as taking an oral pill. But for diseases such as diabetes, chronic pains, post-surgery syndromes, direct dosage of the drug in the body is required. This requires alternate means of precise drug delivery and monitoring. Infusion pumps, introduced in the $80 \mathrm{~s}$, launched a new paradigm for continuous drug delivery in controlled amounts and continue to the be the most commonly employed mechanism for fluid metering in implanted or cannula based devices.

Currently infusion pumps are only capable of delivering large fluid volumes and lack fine resolution. For new protein or macromolecule based drugs and regenerative therapies, nanoliter volume fluid control is needed with picoliter precision. Possibility of building drug delivery devices with multiple drugs packaged in a single device bring the idea of a 

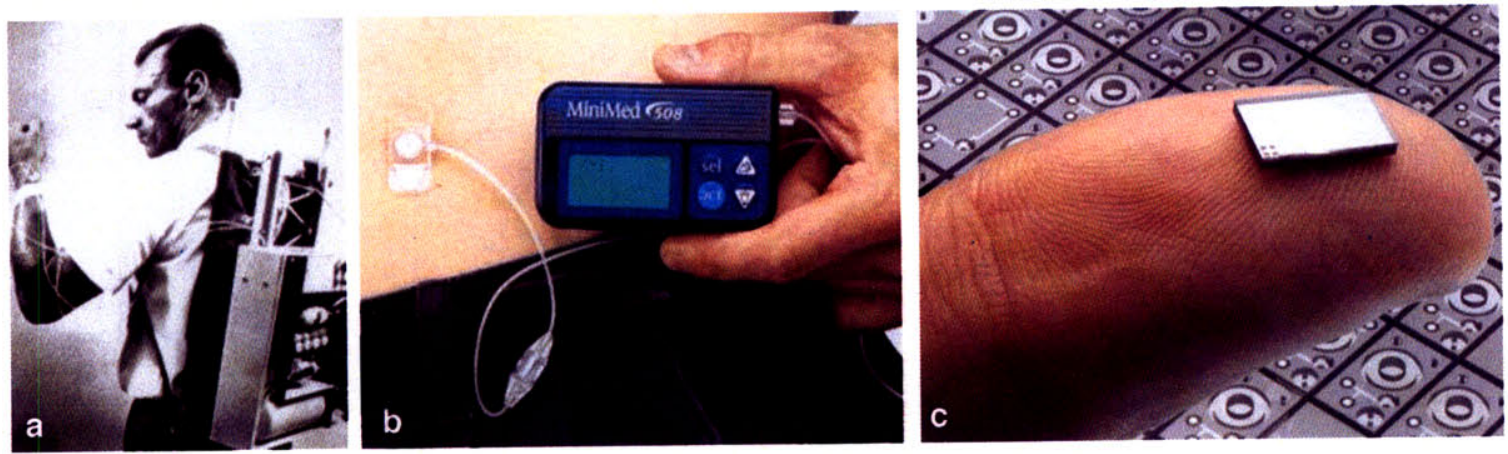

Figure 7-11: Portable mechanical pumps currently used for controlling amount of delivered drug to the body. (a)Early prototype of insulin pump that delivered glucagon (Image courtesy Whitehall Lab, Indiana 1963). (b) Currently used insulin pumps with stepper motor based mechanical means for drug metering. (c) Next generation pump designs for drug delivery manufactured via micro-fabrication techniques. Image courtesy Debiotech Inc.(www.debiotech.com)

pharmacy-on-chip to reality. For miniaturized devices, electronic control has been the only option as yet with limited storage sites, short implant cycle, complex filling and no re-fill procedure for the implant. Using micro-fabrication techniques for mechanical pumps can be manufactured; but they can only deliver hundreds of nano-liter drug dose per actuation cycle, require moving parts for operation and are unsuitable for delivery of drugs in emulsion or powder form.

Bubble logic introduces a means of generation, release and control of nano-liter droplets with targeted drugs: all the required components for a discrete, digital and precise drug delivery through implantable devices. At the heart of such a drug delivery technology is the idea that fluids can also be used to build control circuits analogous to electronics, with pressure as the energy source. These circuits are modular and can be connected to form complex control units like ripple counters, oscillators, flip-flops, and operate at extremely low-Reynolds numbers and can be mass manufactured cheaply from any biocompatible material. Thus a simple ripple counter is capable of binary counting of sub-nanoliter droplets, allowing for a digital control mechanism for metering fixed dosage of multiple drugs over long periods of times. Energy for the operation of the device can also be scavenged from the body itself (for e.g. muscle movement), providing a true implanted device that requires no on-going external maintainence or refills. My near-term goals include targeting a trial drug therapy, 
demonstrating precision, long-term usage and biocompatibility for a bubble logic device with programmable drug metering demonstrated in in-vivo studies.

\subsection{Digital Fabrication}

Fabrication and assembly of multi-material systems can be achieved either by top-down or bottom-up techniques. Top-down approaches allows for fabrication of complex objects to be made from bulk material via controlled processing steps. On the other hand, bottomup approach allows for assembly of large number of parts using directed or self-assembly. Self-assembly techniques suffer from errors that are incorporated during the assembly steps. Also, it is not possible to program the structure of the object to be made. This limits the type of objects that can be fabricated by self-assembly. Directed-assembly can be guided to form the exact shapes in a programmed manner. The current bottleneck in directedassembly exists due to limitations in rapid and precise manipulation of a large number of small parts (conventionally achieved using pick-and-place robots). Thus the throughput from a directed-assembly techniques is low. To form complex parts, the capability to handle a very large number of parts to be assembled in a programmable manner is required. Such an approach is commonly termed as algorithmic-assembly.

Bubble logic provides us with a platform to perform both computation and material processing in a highly integrated platform. Thus not only can materials be arranged in specific structure or geometry, the assembly process can be programmatically controlled by a set of finite state machine running the assembly process. Thus a complex state machine encodes for the structure which is dynamically constructed. Thus bubble logic can provide an integrated platform for performing programmable assembly of materials.

On-chip micro-assembly of a large number of parts can also be performed, with parts arriving at various geometries at specified times. Precise control of hydrodynamic force fields using bubble logic allows for controlled interaction amongst parts. These long and short distance interactions between various micro-parts allow them to join together in a specified manner. The logic operation ensures the sequence of assembly is controlled, thus allowing only a 


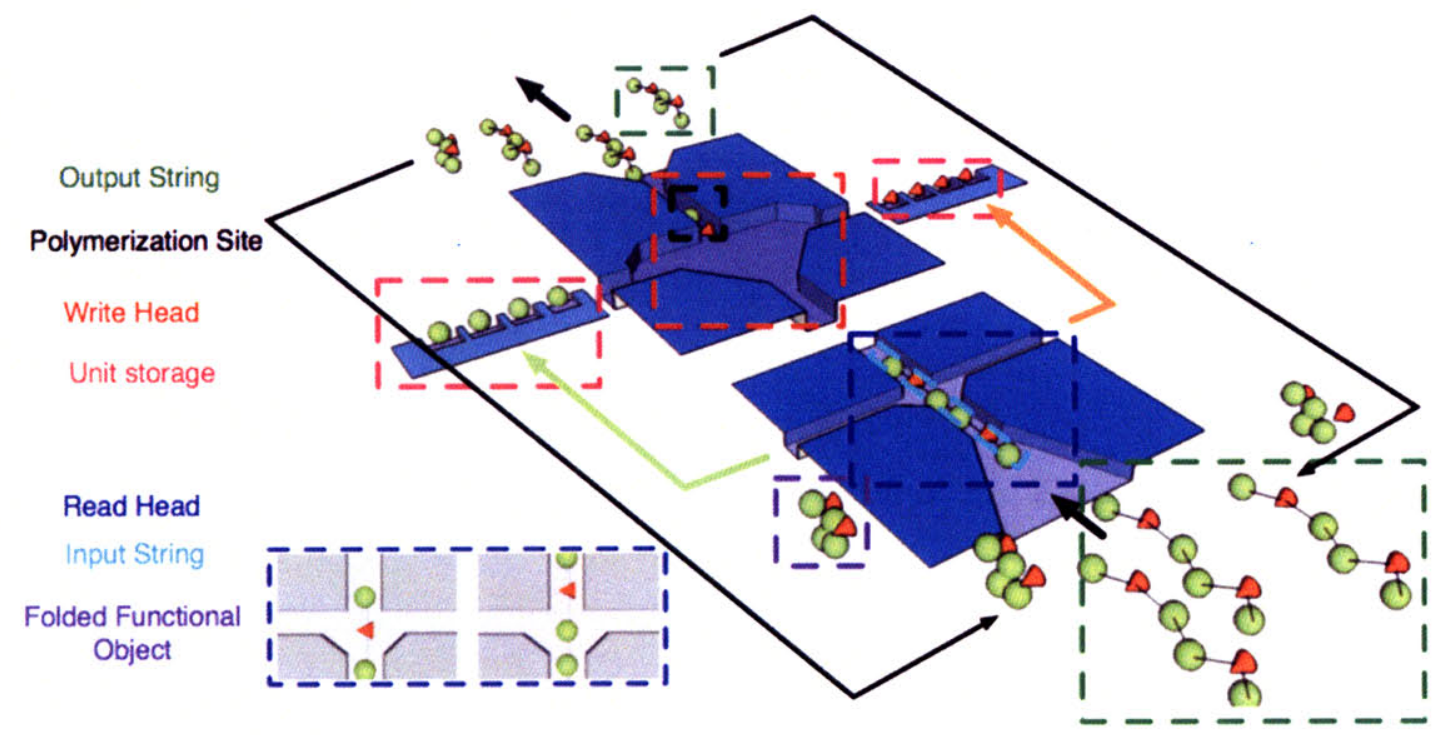

Figure 7-12: Programmable microfluidic assembly and replication of droplet strings. Bubble logic devices, with programmable control of hydrodynamic flow fields provide a platform to fabricate and replicate string like microscopic chains.

single unique geometry to be formed. This approach solves the long standing problem in self-assembly, where a large number of possible interactions allow for error propagation in the assembly process.

A bubble logic directed assembly process might consist of a generic parts library, logic control circuits and assembly chambers with controlled hydrodynamic fields. A hierarchy of sub-assemblies can also be stored for later use. We can further exploit such a platform for programmable assembly to handle micro-scale one-dimensional strings (see figure 7-12).

Bubble logic provides programmable internal control of hydrodynamic flow fields, not achievable by external control techniques. Furthermore, the sensing, actuation and computation required for programmable matter are integrated. These properties make possible a fluidic equivalent of a ribosome, allowing both replication and translation of 1D strings. The flow is driven by a pressure differential as a power source. The fluidic geometry consists of a fluidic read head that reads string like objects and a write head for synthesis of 1D strings. Furthermore, parallel implementation of a fluidic ribosome will enable high-throughput on- 
demand production of not only a single given part but also exponential replication of the same.

\subsection{Future directions}

In addition to next steps listed in this thesis, here I sketch a roadmap for future development of sub-nanoliter fluid handling technologies (see figure 7-13). What is required is development of a design tool that entails building automated software tools for computer aided design, analysis and synthesis of integrated microfluidic control systems. This would allow a modular approach towards building systems with sub-parts that can be shared between various designs and common libraries. Furthermore, using the reduced order modeling techniques, simulation based analysis can be integrated in the design tools to further enhance the reliability and performance of microfluidic devices even before any fabrication is performed.

Further work will be done on device physics and exploring fundamental fluid mechanics at microscopic length scales using the tools proposed. Such an approach will further enable discovery of new mechanisms that can be exploited for new devices. This effort would further allow scaling down of feature sizes and enable exploration of fundamental limits in scaling for the device family proposed in this thesis. Furthermore, bubble logic also provides a basic platform for exploring several novel principles of interfacial fluid mechanics at small length scales.

Identification of best-fit applications for development of a new class of tools is often necessary. Sub-nanoliter fluid handling technologies will find wide array of applications, ranging from high throughput screening, development of chemical libraries, combinatorial chemistry, chemical synthesis, single cell platforms and diagnostics tools. The effort also allows for the integration of analysis and read-out techniques that can be incorporated with sample processing described before. The work of initial array of applications also promotes further development of the basic toolbox of integrated fluid handling and control- introducing new features in the current set of capabilities. 


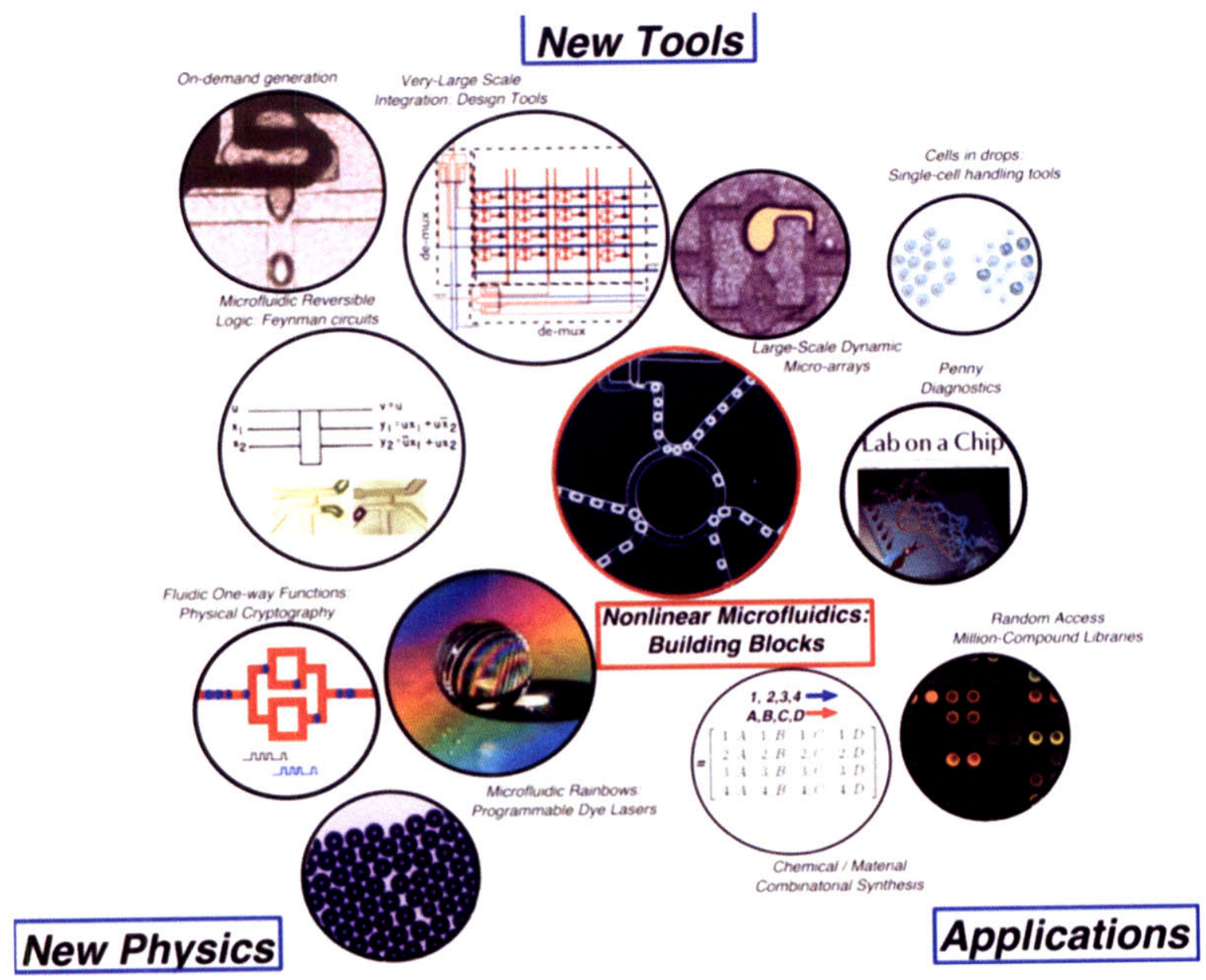

Figure 7-13: Next steps for development of bubble logic. The three efforts are focused on tool development, exploring new physics in these devices and applying the principles learned to various domains of biology, chemistry and material science. 


\subsection{Conclusion}

The field of microfluidics started when tools for microfabrication, initially developed for the micro-electronics industry, became more widely available to biologists, physicists, engineers and alike. With analytical and experimental efforts, tremendous development in our understanding of physical fluid dynamics at small length scale led to an array of applications of microfluidics in biology, analytical chemistry, environmental and material science. The field still holds vast unmet potential to change how quantitative analysis is done in physical sciences. Though benefits of microfluidics for the study of biological and chemical processes have been well established, adoption of this new technology is slower than previously anticipated - both in academia and in the industry.

This thesis proposes a new set of tools for sub-nanoliter fluid handling in integrated microfluidic processors. The implementation of logic in physical fluid dynamics brings the control systems for complex microfluidic processes on-chip. I believe, such an approach, integrating the control systems with the chemistry it controls; provides a scalable means to target complex processes that could not be achieved by current flow control technologies. Thus computation in microfluidics provides a general purpose mechanism for programmable liquid handling on micro-scale devices.

Progress in the field of fluidic logic in the 60's was primarily halted because of well known length-scale issue. As the devices shrink down in size, it is difficult to maintain high Reynolds number limits required for operation of devices dependent on inertial regime. I show that non-inertial non-linearities in low Reynolds number newtonian fluids are far better suited for implementing logic, bistability, cascadability, feedback and error-correction in microfluidic devices. Since the device physics is only dependent on fluid properties, the devices can be fabricated out of a vast array of materials with no significant effect on device

operation. This material independence is a significant advantage for any computational scheme since the devices could potentially handle harsher environmental conditions. Propagation time of milli-seconds was achieved in logic devices implemented in bubble logic. This provides a proof of concept of $\mathrm{kHz}$ range operating frequencies. Though, nowhere 
compared to operating frequencies for electronic counterparts, this is significantly faster than other schemes implementing fluidic logic and is already an order of magnitude faster than solenoid based switching valves which currently operate at hundreds of Hertz.

This thesis also brings the physical and the digital world closer. What is the role of computation in fabrication? How do living organisms construct themselves from a set of instructions? These questions stretch the limits of our understanding of how biological systems replicate, grow and heal themselves over time. My work makes bits physical thus allowing computation to fabricate physical systems. It further suggests a new approach for digitizing fabrication - where programmability of the digital world can be extended to the physical world $[27,28,18,17,9]$.

This thesis describes a system where bits can transport materials as well as information. Thus programs can not only describe things but be things [9]. Assembly of physical objects can be programmed by executing a set of instructions on the computational substrate, merging computation and fabrication. Instead of a computer controlling conventional fabrication tools, in this scenario, the computer is the tool [9].

Previously, it was known that bit representation is always physical, but never before has this principle been exploited for programming the physical world. Bubble logic removes the distinction between materials and mechanisms to control them. This notion of computing to build complex physical things, where the process control is explicitly defined as the operations executed on a processor, brings computer science closer to the domain of physical sciences. The insight that "bits are things" also offers promise in a very wide range of applications - from high throughput genomics and biotechnology to fluidic control systems for soft robotics, printing and digital fabrication. 


\section{Bibliography}

[1] Kepecs A, Uchida N, and Mainen ZF. The sniff as a unit of olfactory processing. Chemical senses, 31:167-179, 2006.

[2] J Anker, C Behrend, and R Kopelman. Aspherical magnetically modulated optical nanoprobes (magmoons). Journal of Applied Physics, Jan 2003.

[3] J Anker and R Kopelman. Magnetically modulated optical nanoprobes. Appl. Phys. Lett., Jan 2003.

[4] Craven BA, Neuberger T, Paterson EG, Webb AG, Josephson EM, Morrison EE, and Settles GS. Reconstruction and morphometric analysis of the nasal airway of the dog (canis familiaris) and implications regarding olfactory airflow. Anat Rec, 290:1325$1340,2007$.

[5] Steve N. Bailey, Randy Z. Wu, and David M. Sabatini. Applications of transfected cell microarrays in high-throughput drug discovery. Drug Discovery Today, 7:113-118, 2002.

[6] F. P. Bretherton. The motion of long bubbles in tubes. Journal of Fluid Mechanics, 10:166-188, 1961.

[7] Brossard Chantal, Rousseau Florence, Llamas Genevive, and Dumont Jean-Pierre. Odor perception over liquid emulsions containing single aroma compounds: Effects of aroma concentration and oil volume fraction. Journal of sensory studies, 17:511-525, 2002 . 
[8] Petra S. Dittrich, Michael Jahnz, and petra Schwille. A new embedded process for compartmentalized cell-free protein expression and on-line detection in microfluidic devices. ChemBioChem, 6:811-814, 2005.

[9] Neil Gershenfeld. The Coming Revolution on Your Desktop: From Personal Computers to Personal Fabrication. Basic Books, 2005.

[10] R.F. Ismagilov, J.M.K. Ng, P.J.A. Kenis, and G.M. Whitesides. Microfluidic arrays of fluid-fluid diffusional contacts as detection elements and combinatorial tools. Analytical Chemistry, 73(21):5207-5213, 2001.

[11] Yoshikuni Kikutani, Takayuki Horiuchi, Kenji Uchiyama, Hideaki Hisamoto, Manabu Tokeshia, and Takehiko Kitamori. Glass microchip with three-dimensional microchannel network for $2 \times 2$ parallel synthesis. Lab on Chip, 2:188-192, 2002.

[12] J. R. Kovac and J. Voldman. Intuitive, image-based cell sorting using optofluidic cell sorting. Analytical Chemistry, 79:9321-9330, 2007.

[13] Mozell MM and Jagodowicz M. Chromatographic separation of odorants by the nose: retention times measured across in vivo olfactory mucosa. Science, pages 1247-1249, 1973.

[14] D.A. Parry. Spider hydraulics. Endeavor, 19:156-162, 1960.

[15] D.A. Parry and R.H.J. Brown. The Hydraulic Mechanism of the Spider Leg. J Exp Biol, 36(2):423-433, 1959.

[16] Manu Prakash and Neil Gershenfeld. Microfluidic bubble logic. Science, 315:832-835, 2007.

[17] Paul W.K. Rothemund. Theory and Experiments in Algorithmic Self-Assembly. PhD thesis, University of Southern California, 2001.

[18] Paul W.K. Rothemund, Nick Papadakis, and Erik Winfree. Algorithmic self-assembly of dna sierpinski triangles. PLoS Biology, 2(12):e424, 2006. 
[19] Thomas A. Schoenfeld and Thomas A. Cleland. Anatomical Contributions to Odorant Sampling and Representation in Rodents: Zoning in on Sniffing Behavior. Chem. Senses, 31(2):131-144, 2006.

[20] Brigitte Stadler, Marta Bally, Dorothee Grieshaber, Janos Voros, Alain Brisson, and H. Michelle Grandin. Creation of a functional heterogeneous vesicle array via dna controlled surface sorting onto a spotted microarray. Biointerphases, 1(4):142-145, 2006.

[21] Anette Stro1mberg, Anders Karlsson, Frida Ryttse, Maximilian Davidson, Daniel T. Chiu, and Owe Orwar. Microfluidic device for combinatorial fusion of liposomes and cells. Analytical Chemistry, 73:126-130, 2001.

[22] Justus Verhagen, Daniel Wesson, Theoden Netoff, John White, and Matt Wachowiak. Sniffing controls an adaptive filter of sensory input to the olfactory bulb. Nat Neurosci, 10(5):631-639, 2007. 10.1038/nn1892.

[23] J Voldman, M Toner, ML Gray, and MA Schmidt. A dielectrophoresis-based array cytometer. Transducers, pages 322-325, 2001.

[24] A Vuppu, A Garcia, M Hayes, K Booksh, and P Phelan. Phase sensitive enhancement for biochemical detection using rotating paramagnetic particle chains. Journal of Applied Physics, Jan 2004.

[25] Wei-HeongTan and Shoji Takeuchi. A trap-and-release integrated microfluidic system for dynamic microarray applications. Proc. Nat. Acad. of Sci., 104:1146-1151, 2007.

[26] Wei-HeongTan and Shoji Takeuchi. Dynamic microarray system with gentle retrieval mechanism for cell encapsulating hydrogel beads. Lab on a Chip, 7:259-266, 2008.

[27] Erik Winfree. Algorithmic Self-Assembly of DNA. PhD thesis, Caltech, 1998.

[28] Erik Winfree. Self-healing tiles. Nanotechnology: Science and Computation, pages 55-78, 2006. 
[29] Kai Zhao, Pamela Dalton, Geoffery C. Yang, and Peter W. Scherer. Numerical Modeling of Turbulent and Laminar Airflow and Odorant Transport during Sniffing in the Human and Rat Nose. Chem. Senses, 31(2):107-118, 2006. 